\title{
2-D Triangular Lattice Photonic Crystal Low Loss Waveguide Bends and Y Splitter at Microwave Frequencies
}

\author{
By \\ Deepak Kaushal \\ B.Tech. (HPU)
}

\begin{abstract}
A Thesis
Submitted to the Faculty of Graduate Studies and Research in Partial Fulfillment of the Requirements for the Degree of Master of Applied Science in Electrical and Computer Engineering
\end{abstract}

Ottawa-Carleton Institute for Electrical Engineering

Department of Electronics

Carleton University

Ottawa, Canada

Copyright (C April 2013 by Deepak Kaushal 
Library and Archives

Canada

Published Heritage

Branch

395 Wellington Street

Ottawa ON K1A ON4

Canada
Bibliothèque et

Archives Canada

Direction du

Patrimoine de l'édition

395 , rue Wellington

Ottawa ON K1A ON4

Canada
Your file Votre référence

ISBN: 978-0-494-94641-1

Our file Notre référence

ISBN: 978-0-494-94641-1
NOTICE:

The author has granted a nonexclusive license allowing Library and Archives Canada to reproduce, publish, archive, preserve, conserve, communicate to the public by telecommunication or on the Internet, loan, distrbute and sell theses worldwide, for commercial or noncommercial purposes, in microform, paper, electronic and/or any other formats.

The author retains copyright ownership and moral rights in this thesis. Neither the thesis nor substantial extracts from it may be printed or otherwise reproduced without the author's permission.
AVIS:

L'auteur a accordé une licence non exclusive permettant à la Bibliothèque et Archives Canada de reproduire, publier, archiver, sauvegarder, conserver, transmettre au public par télécommunication ou par l'Internet, prêter, distribuer et vendre des thèses partout dans le monde, à des fins commerciales ou autres, sur support microforme, papier, électronique et/ou autres formats.

L'auteur conserve la propriété du droit d'auteur et des droits moraux qui protege cette thèse. $\mathrm{Ni}$ la thèse ni des extraits substantiels de celle-ci ne doivent être imprimés ou autrement reproduits sans son autorisation.
In compliance with the Canadian Privacy Act some supporting forms may have been removed from this thesis.

While these forms may be included in the document page count, their removal does not represent any loss of content from the thesis.
Conformément à la loi canadienne sur la protection de la vie privée, quelques formulaires secondaires ont été enlevés de cette thèse.

Bien que ces formulaires aient inclus dans la pagination, il n'y aura aucun contenu manquant. 


\begin{abstract}
A photonic crystal $(\mathrm{PhC})$ is a low loss periodic dielectric medium that prevents the flow of light in certain directions for specified frequencies. The availability of low loss waveguide bends in photonic crystal structures makes possible numerous integrated optic devices. The method proposed in this thesis consists of introducing a dielectric shift equivalent to a shear displacement along a segment of the $\mathrm{PhC}$ waveguide and results in a double bend waveguide. The degree of sheer determines the waveguide inclination angle and this angle can extend up to several degrees. As a result of the sheer, the input and output segments of the waveguides are laterally offset relative to each other but remain parallel. Theoretical analysis is performed using FDTD and PWM and predicts low loss bends in both the square and triangular arrays composed of high dielectric constant rods in an air background. Micro-fabrications of complex photonic crystal are quite difficult to achieve but physical models can easily be constructed at the microwave regime at a much larger length scale of centimeters. Experiments are performed in the microwave regime using an array of alumina rods in the air over the 2 to $14 \mathrm{GHz}$ range. In particular detailed experiments were performed for the TM polarization for the triangular lattice in order to obtain information on bend loss versus sheer displacement and waveguide offset angle and show that low losses are achievable for deflection up to 15 degrees. Additional experimental results indicate that a low loss $\mathrm{Y}$ splitter can also be produced using the same technique.
\end{abstract}




\section{Acknowledgment}

I would like to express my deep appreciation to my supervisor, Dr. Robert Gauthier, whose support, encouragement, creativity, and insight have steered me through tough situations positively, and helped me to develop my critical thinking skills. I am extremely fortunate to be his research student. His mentoring style, guiding rather than leading, has allowed me to spread my intellectual wings and tackle problems on my own.

My utmost gratitude, thanks, and appreciation go to my parents and in-laws, for their never-ending support, kindness, and belief in me; and for simply being the kind-hearted people they are, to whom I will always be indebted.

I would like to acknowledge the love and support of my wife, Kamna Kaushal, especially her continuous encouragement, and constant sacrifices. To her, I extend my gratitude and appreciation for putting up with my unusual work schedule and for remaining patient through the difficult periods of my research. I would also like to express thanks to my son, Akarshit Kaushal, who always supported his mother during times I was away.

Thank you to staff members in the electronics department, especially Blazenka Power and Anna Lee, for the valuable support and help.

A special thanks to Nagui Mikhail, who helped me finish my experimental work in the microwave laboratory on time. I am also grateful to the machine shop staff, especially Alexander Proctor for his valuable contribution to the completion of the template-designing task. 
I had the pleasure of working with genuinely nice colleagues, especially Scott Newman and Mohammed Alzahrani: thank you for your valuable suggestions, help and support during the course of the work.

Last but not least, I am very grateful for the encouragement provided by my friends who, even though not always with me, kindly offered their assistance and support on many occasions. 


\section{Table of Contents}

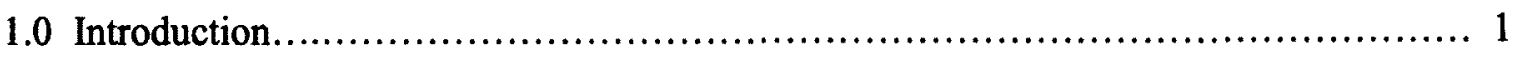

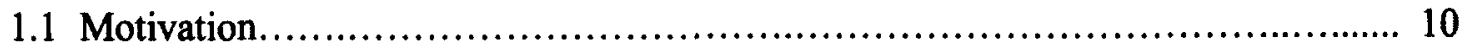

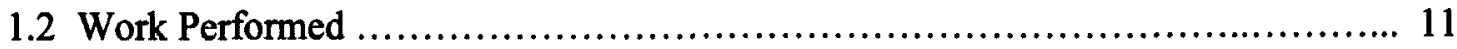

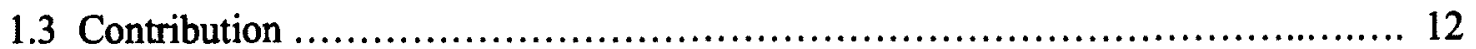

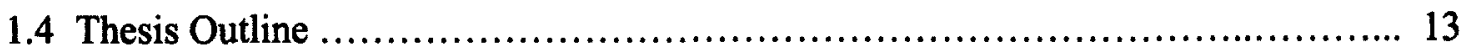

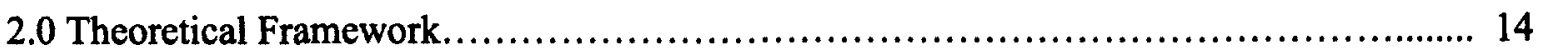

2.1 Master Equation........................................................ 14

2.2 2-D Photonic Crystal Wave Equation for TM and TE Polarization.......................... 20

2.2.1 TM Polarization ..................................................... 21

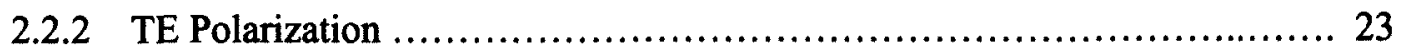

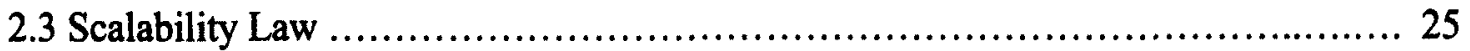

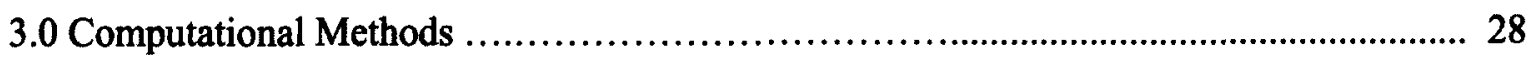

3.1 Plane Wave Method: Two Dimensional Photonic Crystal................................. 29

3.1.1 Photonic Band Structure for a 2-D Photonic Crystal: TM Polarized Mode............... 41

3.1.2 Photonic Bandgap Maps..................................................... 43

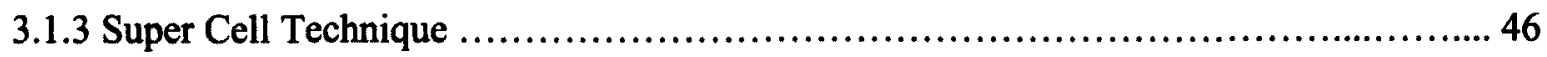

3.2 Finite Difference Time Domain(FDTD) Method............................... 49 
3.2.1 Formulation of finite difference time domain (FDTD) method........................ 50

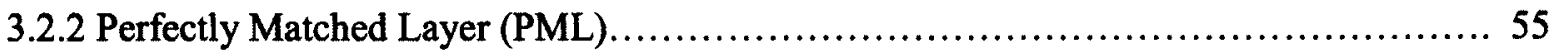

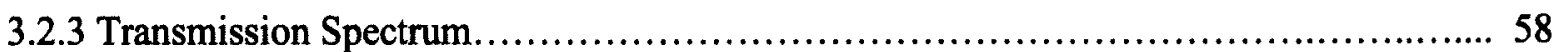

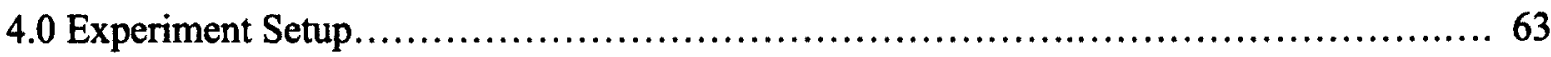

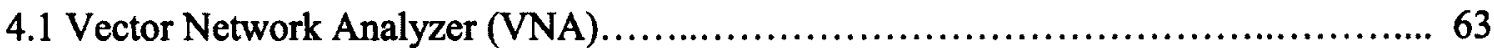

4.1.1 VNA Calibration Procedure............................................. 66

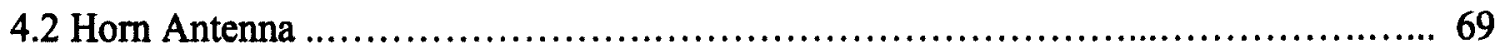

4.3 Microwave Measurement Without 2-D Photonic Crystal Model....................... 70

4.4 Alumina Rod: Specifications and Characteristics................................. 72

4.5 Design Parameters for 2-D Photonic Crystal Model - Microwave Regime ............. 75

4.6 2-D Photonic Crystal Template Design ....................................... 76

4.7 Microwave Measurement for 2-D Photonic Crystal (Without and With Waveguide)..... 81

5.0 Double Bend Waveguide Measurements and Y Branch Structure......................... 85

5.1 Double Bend Waveguide Formation.............................................. 85

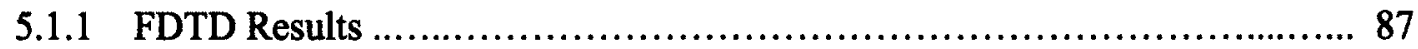

5.1.2 Microwave Experimental Results.................................. 89 
5.3 Y splitter 108

6.0 Conclusion

6.1 Potential Application of Y splitter 115

6.2 Future Directions 116

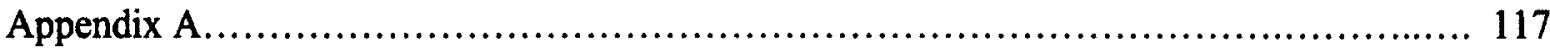

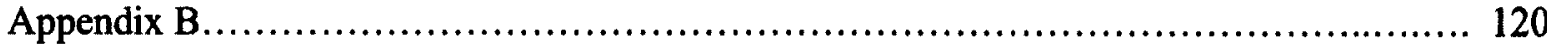

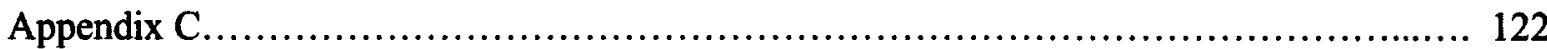

Appendix D.................................................................. 153

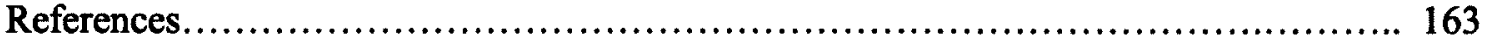




\section{List of Figures}

Figure 1.1: Schematic representation of multidimensional (1-D, 2-D, and 3-D) photonic crystals showing the periodicity of photonic crystals in one or more axes. The different colors demonstrate that a photonic crystal contains different materials with different dielectric constants. ${ }^{1}$

Figure 1.2: TM Polarization (left side): the magnetic field component $\left(H_{x}, H_{y}\right)$ confined to $(\mathrm{x}$, y) plane and TE Polarization (right side): the electric field component $\left(E_{x}, E_{y}\right)$ confined to $(x, y)$ plane.

Figure 1.3: Defects in 2-D photonic crystal (square and triangular lattice), (a) a cavity (point) defect (b) an extended (line) defect.

Figure 1.4: A 2-D photonic crystal (square lattice) linear waveguide with a sharp bend comer $\left(90^{\circ}\right)$

Figure 1.5: A linear waveguide ${ }^{26}$ with double bend where input and output waveguide segments are maintained parallel with an inclination angle $(\theta)$ varied from $0^{\circ}$ to $20^{\circ}$.

Figure 1.6: A 2-D photonic crystal linear waveguide with a square lattice, (a) $\mathrm{Y}$ branch, (b) $\mathrm{T}$ branch.

Figure 2.1: Square (left) and triangular (right) lattice photonic crystals. The gray circles represent alumina rods with relative dielectric constant; ' $\varepsilon_{\mathrm{Al}}$ ' and rod radius; ' $r$ '. The 
lattice constant is ' $a$ '. The space between rods in white represent air with relative dielectric constant; ' $\varepsilon_{\text {air }}$ '.

Figure 3.1: A 2-D photonic crystal with square lattice of columns of high dielectric constant, ' $\varepsilon_{\mathrm{Al}}$ ' (black dots) surrounded in a background of low dielectric constant, ' $\varepsilon_{\mathrm{air}}$ ' (white region) (a) real space arrangement with two primitive lattice vectors $\vec{a}_{1}$, and $\vec{a}_{2}$; (b) reciprocal space with two reciprocal lattice vectors $\vec{b}_{1}$ and $\vec{b}_{2}$; the Brillouin zone is outlined by a solid bold border and the black shaded triangular region with points ГXM represents the irreducible $1 / 8$ Brillouin zone.

Figure 3.2: A 2-D photonic crystal with a triangular lattice of columns of high dielectric constant, ' $\varepsilon_{\mathrm{Al}}$ ' (black dots) surrounded in a background of low dielectric constant, ' $\varepsilon_{\text {air }}$ ' (white region) (a) real space arrangement with two primitive lattice vectors $\vec{a}_{1}$ and $\vec{a}_{2}$; (b) reciprocal space with two reciprocal lattice vector $\left(\vec{b}_{1}\right.$ and $\left.\vec{b}_{2}\right)$; the Brillouin zone outlined by a solid bold border and the shaded triangular region with points $\Gamma K M$ represent the irreducible $1 / 12$ Brillouin zone.

Figure 3.3: The photonic band structure of the two-dimensional triangular lattice of dielectric columns (TM polarized mode). The photonic bandgap indicated in the figure shows the range of forbidden frequencies. Inset shows arrangement of alumina rods in a triangular lattice.

Figure 3.4: Plot of normalized frequency, $\left(\frac{\omega a}{2 \pi c}\right)$ versus lattice constant ' $a$ '. Photonic bandgaps (black) are shown for a two-dimensional photonic crystal (TM polarization) with triangular lattice and dielectric rods $\left(\varepsilon_{\mathrm{Al}}=9.2\right)$ with a background as air $\left(\varepsilon_{\mathrm{air}}=1\right)$. 
Rod radius is constant, ' $r=0.1 \mu \mathrm{m}$ ' and the chosen value of the lattice constant, ' $a=$ $0.70 \mu \mathrm{m}^{\prime}$.

Figure 3.5: An array of 2-D triangular lattice photonic crystal with a line defect in the $\mathrm{X}$ direction. The shaded region represents the supercell.

Figure 3.6: $\Gamma-\mathrm{X}$ dispersion curve of 2-D line defect waveguide photonic crystal with triangular lattice having lattice constant, $a=0.70 \mu \mathrm{m}, \mathrm{TM}$ polarization obtained with the supercell technique. Single guided modes at normalized frequencies between $0.58-$ 0.74 within the photonic bandgap is present.

Figure 3.7: Spatial arrangement of field variables in the FDTD grid for two dimensions (TM polarized).

Figure 3.8: A 2-D triangular lattice photonic crystal place in the FDTD environment in order to determine the transmission spectrum. Alumina rod (black dots), radius, $r=0.1 \mu \mathrm{m}$ with dielectric constant, $\left(\varepsilon_{\mathrm{Al}}=9.2\right)$ with a background (white) as air $\left(\varepsilon_{\text {air }}=1\right)$ and lattice constant, $\mathrm{a}=0.70 \mu \mathrm{m}$.

Figure 3.9: Transmission spectrum obtained from the FDTD simulator for a triangular array photonic crystal (TM polarization) after 100,000 iterations. A bandgap obtained at wavelength range of, $\lambda=1.28 \mu \mathrm{m}$ to $2.02 \mu \mathrm{m}$.

Figure 3.10: A 2-D linear waveguide formed by removing the $8^{\text {th }}$ row of alumina rods in the triangular lattice. Alumina rod (black dots), radius, $r=0.1 \mu \mathrm{m}$ with dielectric 
constant, $\left(\varepsilon_{\mathrm{Al}}=9.2\right)$ with a background (white) as air $\left(\varepsilon_{\mathrm{air}}=1\right)$ and lattice constant, a $=0.70 \mu \mathrm{m}$.

Figure 3.11: Transmission spectrum for a 2-D linear waveguide with triangular lattice obtained from the FDTD simulator after 100,000 iterations (TM polarization). The spectrum shows the presence of high transmission within the original bandgap region indicating that one or more modes can be propagated.

Figure 4.1: Measurements performed with the VNA, (a) transmission measurement, (b) reflection measurement; the inset shows the pictorial view of the HP 8720 ES Sparameter Vector Network Analyzer- $(50 \mathrm{MHz}-20 \mathrm{GHz})$

Figure 4.2: Two port S-parameters $\left(S_{11}, S_{21}, S_{22}, S_{12}\right)$ for the Vector Network Analyzer (VNA)

Figure 4.3: Calibration of full two-port network analyzer with standard connection as per the manufacturer's instructions.

Figure 4.4: Pictorial view of two horn antennas (a) model H-1498 as receiver (b) model EM 6961 as a transmitter used in the microwave experiments.

Figure 4.5: Two horn antennas (transmitter and receiver) with a distance of $300 \mathrm{~mm}$ was connected to a 2 port vector network analyzer with TM polarization and transmission power; $S_{21}(d B)$ was obtained without placing a 2-D photonic crystal model in between them.

Figure 4.6: A reference transmission curve obtained when the transmitter and receiver (hom antennas) were placed at a distance of $300 \mathrm{~mm}$. 
Figure 4.7: Statistical analysis (bell-shaped curve) of the measurements (1,500 readings) made on 300 circular alumina rods with the help of a digital calliper to get the final rod diameter, $\mathrm{d}=0.485 \mathrm{~cm}$.

Figure 4.8: A 2-D photonic crystal template with a triangular lattice array consisting of three main sections with overall dimension of $299.1 \mathrm{~mm}$ X $569.8 \mathrm{~mm}$ having a total of 17 periods in the $\mathrm{Y}$ direction and 62 periods in the $\mathrm{X}$ direction. The linear waveguide is formed by removing the ninth row in the $\mathrm{X}$ direction, the waveguide channel width, $\mathrm{W}=24.45 \mathrm{~mm}$.

Figure 4.9: Section 1 - Fixed part with overall dimension of $299.1 \mathrm{~mm}$ X $178.7 \mathrm{~mm}$ consisting of 17 periods $(239.1 \mathrm{~mm})$ in the $\mathrm{Y}$ direction and 19 periods $(158.7 \mathrm{~mm})$ in the $\mathrm{X}$ direction to accommodate up to 152 alumina rods.

Figure 4.10: One of 16 strips, type 1 , containing 8 holes of diameter, $d=4.85 \mathrm{~mm}$ and the distance from center to center of hole was $29.3 \mathrm{~mm}$. The overall dimension of the strips is $299.1 \mathrm{~mm} \mathrm{X8.75} \mathrm{mm} \mathrm{to} \mathrm{accommodate} 128$ alumina rods.

Figure 4.11: One of 16 strips, type 2, containing 9 holes of diameter, $d=4.85 \mathrm{~mm}$ and the distance from center to center of hole was $29.3 \mathrm{~mm}$. The overall dimension of the strips is $299.1 \mathrm{~mm} \mathrm{X} 8.75 \mathrm{~mm}$ to accommodate 144 alumina rods.

Figure 4.12: Section 3 - A fixed part with overall dimension of $299.1 \mathrm{~mm}$ X $111.1 \mathrm{~mm}$ consisting of 17 periods $(239.1 \mathrm{~mm})$ in the $Y$ direction and 11 periods $(91.1 \mathrm{~mm})$ in the $\mathrm{X}$ direction to accommodate upto 94 alumina rods. 
Figure 4.13: Picture of the alumina rods arranged in the triangular lattice using the base templates (lattice constant, $\mathrm{a}=16.9 \mathrm{~mm}$ ).

Figure 4.14: Transmission curves with and without a 2-D photonic crystal model present. The comparison of the curves shows the presence of the bandgap from $6.50 \mathrm{GHz}$ to $11.72 \mathrm{GHz}$ when the structure is in place.

Figure 4.15: Picture of a 2-D linear waveguide within the triangular lattice. The waveguide has width, $\mathrm{W}=24.45 \mathrm{~mm}$.

Figure 4.16: Transmission spectrum obtained in the microwave regime when the triangular array is fully populated (bandgap) and when one row of rods is removed (waveguide). The reference trace is obtained by taking a spectrum with the rod array fully removed.

Figure 5.1: The waveguide configuration with double bend present. Lattice is triangular with high dielectric rods shown in black and air in background in white. The bend in the waveguides is parameterized based on the inclination angle between input and shifted photonic crystal segments.

Figure 5.2: Plot between transmission loss $(\mathrm{dB})$ versus waveguide bend angle, $(\theta)$ obtained after analyzing transmission spectrums at wavelength, $\lambda=1.45 \mu \mathrm{m}$, with different waveguide bend angles, $\left(\theta=0^{\circ}\right.$ to $\left.30^{\circ}\right)$.

Figure 5.3: Transmission spectrum for a linear waveguide with lattice constant, $a=16.9 \mathrm{~mm}$ at bend angle, $\theta=0^{\circ}$ showing the photonic bandgap ( $\mathrm{f}=6.5 \mathrm{GHz}$ to $11.72 \mathrm{GHz}$ ) with a 
range of frequencies ( $\mathrm{f}=7.40 \mathrm{GHz}$ to $8.81 \mathrm{GHz}$ ) that is getting through the waveguide channel.

Figure 5.4: Picture of the 2-D photonic crystal model showing double bends at $\theta=10^{\circ}$ with an incremental shear section between the input and output sections.

Figure 5.5: (a-o) shows transmission curves (transmission power (dB) versus frequency (GHz)) for different waveguide offset angle $\left(1^{\circ}\right.$ to $\left.15^{\circ}\right)$. Referred to Appendix $\mathrm{C}$ for full page view.

Figure 5.6: (a-o) shows transmission curves (transmission power $(\mathrm{dB})$ versus frequency $(\mathrm{GHz})$ ) for different waveguide offset angle $\left(16^{\circ}\right.$ to $\left.30^{\circ}\right)$. Referred to appendix $\mathrm{C}$ for full page view.

Figure 5.7: 3-D line plot showing the transmission power for a range of frequency $(7 \mathrm{GHz}$ to 9.26 $\mathrm{GHz}$ ) at different waveguide bend angles, $\theta$ varying from $0^{\circ}$ to $30^{\circ}$.

Figure 5.8 (a): 3-D line plot for a frequency range of $7.88 \mathrm{GHz}$ to $8.03 \mathrm{GHz}$ showing a relative transmission loss at different waveguide bend angle, $\theta$, varying from $0^{\circ}$ to $30^{\circ}$.

Figure 5.8 (b): Plot showing the average transmission loss for a frequency interval $(7.88 \mathrm{GHz}$ to $8.03 \mathrm{GHz}$ ) at different waveguide bend angle, $\theta$, varying from $0^{\circ}$ to $30^{\circ}$.

Figure 5.9 (a): 3-D line plot for a frequency range of $8.45 \mathrm{GHz}$ to $8.81 \mathrm{GHz}$ showing a relative transmission loss at different waveguide bend angle, $\theta$, varying from $0^{\circ}$ to $30^{\circ}$.

Figure 5.9 (b): Plot showing the average transmission loss for a frequency interval $(8.45 \mathrm{GHz}$ to $8.81 \mathrm{GHz}$ ) at different waveguide bend angle, $\theta$, varying from $0^{\circ}$ to $30^{\circ}$. 
Figure 5.10 (a): 3-D line plot for a frequency range of $8.54 \mathrm{GHz}$ to $8.81 \mathrm{GHz}$ showing a relative transmission loss at different waveguide bend angle, $\theta$, varying from $0^{\circ}$ to $30^{\circ}$.

Figure 5.10 (b): Plot showing the average relative transmission loss versus waveguide for a frequency window of $8.54 \mathrm{GHz}$ to $8.81 \mathrm{GHz}$.

Figure 5.11 (a): 3-D line graph showing transmission efficiency at different waveguide bend angle, $\theta$ for a range of frequency $(8.54 \mathrm{GHz}$ to $8.81 \mathrm{GHz})$.

Figure 5.11 (b): Plot showing the average transmission efficiency for a frequency range ( $f=8.54$ $\mathrm{GHz}$ to $8.81 \mathrm{GHz})$ at different waveguide bend angle $\left(\theta=0^{\circ}\right.$ to $\left.30^{\circ}\right)$ that are guided through a 2-D photonic crystal linear waveguide in microwave regime.

Figure 5.12: Plot relative transmission loss versus waveguide bend angle for the frequency of $8.54 \mathrm{GHz}(\lambda=1.45 \mu \mathrm{m})$.

Figure 5.13: Plot of relative transmission loss versus waveguide bend angle for the theoretical predictions and experimental results at a frequency of $8.54 \mathrm{GHz}(\lambda=1.45 \mu \mathrm{m})$.

Figure 5.14: Sketch of the $Y$ branch splitter produced using the increment dielectric shift process.

Figure 5.15: A complete microwave experimental setup (side view) of 2-D Y branch (1 X 2 splitter) model with triangular lattice with overall dimension of $299.1 \mathrm{~mm} \mathrm{X} 262.45$ mm accommodating 234 alumina rods.

Figure 5.16: Top view of the 2-D photonic crystal Y splitter structure (triangular lattice) with one input and two outputs. 
Figure 5.17: Transmission spectrum for a $Y$ splitter designed with a branch angle, $\theta=5^{\circ}$, in the microwave regime. The split ratio is nearly $50 / 50$ with an overall efficiency of $82 \%$

Figure 5.18: Enlarged section (Figure (5.17)) of the transmission spectrum for a $\mathrm{Y}$ splitter at frequency, $\mathrm{f}=8.54 \mathrm{GHz}$ showing two output waveguides with transmission efficiency of $41 \%$ each.

Figure (A.1): Plot between normalized frequency, $\left(\frac{\omega \mathrm{a}}{2 \pi \mathrm{c}}\right)$ versus lattice constant ' $a$ '. Photonic bandgaps are for a two dimensional photonic crystal (TE polarization) with triangular lattice of dielectric rods $\left(\varepsilon_{\mathrm{Al}}=9.2\right)$ with a background as air $\left(\varepsilon_{\mathrm{air}}=1\right)$. The inset shows the triangular lattice formations of dielectric alumina rods. Rod radius is constant, ' $r=0.1 \mu \mathrm{m}$ ' and expected value of chosen lattice constant, ' $a=0.70$ $\mu \mathrm{m}$ ' corresponding to TM polarization.

Figure (A.2): Plot between normalized frequency, $\left(\frac{\omega \mathrm{a}}{2 \pi \mathrm{c}}\right)$ vs lattice constant ' $\mathrm{a}$ '. A complete photonic bandgap for a two dimensional photonic crystal (TM and TE polarization) with triangular lattice of dielectric rods $\left(\varepsilon_{\mathrm{Al}}=9.2\right)$ with background as air $\left(\varepsilon_{\mathrm{air}}=1\right)$. The inset shows the triangular lattice formations of dielectric alumina rods. Rod radius is constant, ' $r=0.1 \mu \mathrm{m}$ ' and expected value of chosen lattice constant, ' $a=$ $0.70 \mu \mathrm{m}$ ' corresponding to a complete band gap for both TE and TM modes.

Figure B.1: Brief data sheet for different alumina rod composition prepared by Superior Technical Ceramics corp.

Figure C.1: Transmission spectrum for a linear waveguide with lattice constant, $a=16.9 \mathrm{~mm}$ at bend angle, $\theta=1^{\circ}$ 
Figure C.2: Transmission spectrum for a linear waveguide with lattice constant, $a=16.9 \mathrm{~mm}$ at bend angle, $\theta=2^{\circ}$

Figure C.3: Transmission spectrum for a linear waveguide with lattice constant, $a=16.9 \mathrm{~mm}$ at bend angle, $\theta=3^{\circ}$

Figure C.4: Transmission spectrum for a linear waveguide with lattice constant, $a=16.9 \mathrm{~mm}$ at bend angle, $\theta=4^{\circ}$

Figure C.5: Transmission spectrum for a linear waveguide with lattice constant, $a=16.9 \mathrm{~mm}$ at bend angle, $\theta=5^{\circ}$

Figure C.6: Transmission spectrum for a linear waveguide with lattice constant, $a=16.9 \mathrm{~mm}$ at bend angle, $\theta=6^{\circ}$

Figure C.7: Transmission spectrum for a linear waveguide with lattice constant, $a=16.9 \mathrm{~mm}$ at bend angle, $\theta=7^{\circ}$

Figure C.8: Transmission spectrum for a linear waveguide with lattice constant, $a=16.9 \mathrm{~mm}$ at bend angle, $\theta=8^{\circ}$

Figure C.9: Transmission spectrum for a linear waveguide with lattice constant, $a=16.9 \mathrm{~mm}$ at bend angle, $\theta=9^{\circ}$

Figure C.10: Transmission spectrum for a linear waveguide with lattice constant, $a=16.9 \mathrm{~mm}$ at bend angle, $\theta=10^{\circ}$

Figure C.11: Transmission spectrum for a linear waveguide with lattice constant, $a=16.9 \mathrm{~mm}$ at bend angle, $\theta=11^{\circ}$ 
Figure C.12: Transmission spectrum for a linear waveguide with lattice constant, $a=16.9 \mathrm{~mm}$ at bend angle, $\theta=12^{\circ}$

Figure C.13: Transmission spectrum for a linear waveguide with lattice constant, $a=16.9 \mathrm{~mm}$ at bend angle, $\theta=13^{\circ}$

Figure C.14: Transmission spectrum for a linear waveguide with lattice constant, $a=16.9 \mathrm{~mm}$ at bend angle, $\theta=14^{\circ}$

Figure C.15: Transmission spectrum for a linear waveguide with lattice constant, $a=16.9 \mathrm{~mm}$ at bend angle, $\theta=15^{\circ}$

Figure C.16: Transmission spectrum for a linear waveguide with lattice constant, $a=16.9 \mathrm{~mm}$ at bend angle, $\theta=16^{\circ}$

Figure C.17: Transmission spectrum for a linear waveguide with lattice constant, $a=16.9 \mathrm{~mm}$ at bend angle, $\theta=17^{\circ}$

Figure C.18: Transmission spectrum for a linear waveguide with lattice constant, $a=16.9 \mathrm{~mm}$ at bend angle, $\theta=18^{\circ}$

Figure C.19: Transmission spectrum for a linear waveguide with lattice constant, $a=16.9 \mathrm{~mm}$ at bend angle, $\theta=19^{\circ}$

Figure C.20: Transmission spectrum for a linear waveguide with lattice constant, $a=16.9 \mathrm{~mm}$ at bend angle, $\theta=20^{\circ}$

Figure C.21: Transmission spectrum for a linear waveguide with lattice constant, $a=16.9 \mathrm{~mm}$ at bend angle, $\theta=21^{\circ}$ 
Figure C.22: Transmission spectrum for a linear waveguide with lattice constant, $a=16.9 \mathrm{~mm}$ at bend angle, $\theta=22^{\circ}$

Figure C.23: Transmission spectrum for a linear waveguide with lattice constant, $a=16.9 \mathrm{~mm}$ at bend angle, $\theta=23^{\circ}$

Figure C.24: Transmission spectrum for a linear waveguide with lattice constant, $a=16.9 \mathrm{~mm}$ at bend angle, $\theta=24^{\circ}$

Figure C.25: Transmission spectrum for a linear waveguide with lattice constant, $a=16.9 \mathrm{~mm}$ at bend angle, $\theta=25^{\circ}$

Figure C.26: Transmission spectrum for a linear waveguide with lattice constant, $a=16.9 \mathrm{~mm}$ at bend angle, $\theta=26^{\circ}$

Figure C.27: Transmission spectrum for a linear waveguide with lattice constant, $a=16.9 \mathrm{~mm}$ at bend angle, $\theta=27^{\circ}$

Figure C.28: Transmission spectrum for a linear waveguide with lattice constant, $a=16.9 \mathrm{~mm}$ at bend angle, $\theta=28^{\circ}$

Figure C.29: Transmission spectrum for a linear waveguide with lattice constant, $a=16.9 \mathrm{~mm}$ at bend angle, $\theta=29^{\circ}$

Figure C.30: Transmission spectrum for a linear waveguide with lattice constant, $a=16.9 \mathrm{~mm}$ at bend angle, $\theta=30^{\circ}$

Figure D.1: Plot drawn at a particular frequency point, $\mathrm{f}=8.57 \mathrm{GHz}$ with different waveguide bend angle, $\theta$ versus relative transmission loss in microwave regime. 
Figure D.2: Plot drawn at a particular frequency point, $f=8.60 \mathrm{GHz}$ with different waveguide bend angle, $\theta$ versus relative transmission loss in microwave regime.

Figure D.3: Plot drawn at a particular frequency point, $f=8.63 \mathrm{GHz}$ with different waveguide bend angle, $\theta$ versus relative transmission loss in microwave regime.

Figure D.4: Plot drawn at a particular frequency point, $f=8.66 \mathrm{GHz}$ with different waveguide bend angle, $\theta$ versus relative transmission loss in microwave regime.

Figure D.5: Plot drawn at a particular frequency point, $\mathrm{f}=8.69 \mathrm{GHz}$ with different waveguide bend angle, $\theta$ versus relative transmission loss in microwave regime.

Figure D.6: Plot drawn at a particular frequency point, $\mathrm{f}=8.72 \mathrm{GHz}$ with different waveguide bend angle, $\theta$ versus relative transmission loss in microwave regime.

Figure D.7: Plot drawn at a particular frequency point, $\mathrm{f}=8.75 \mathrm{GHz}$ with different waveguide bend angle, $\theta$ versus relative transmission loss in microwave regime.

Figure D.8: Plot drawn at a particular frequency point, $\mathrm{f}=8.78 \mathrm{GHz}$ with different waveguide bend angle, $\theta$ versus relative transmission loss in microwave regime.

Figure D.9: Plot drawn at a particular frequency point, $\mathrm{f}=8.81 \mathrm{GHz}$ with different waveguide bend angle, $\theta$ versus relative transmission loss in microwave regime. 


\section{List of Tables}

Table 4.1: Specifications of two horn antennas use for the microwave experimental purpose.

Table 4.2: Parameter used for experimental design in microwave regime. 


\section{List of Symbols}

\begin{tabular}{|c|c|}
\hline$\lambda$ & Wavelength \\
\hline $\mathrm{f}$ & Frequency \\
\hline$\vec{k}$ & The wave vector \\
\hline$\mu$ & The permeability of the material with subscripts further \\
\hline & specifying its nature: $\mu_{0}-$ freespace and $\mu_{r}-$ relative permeability \\
\hline$\varepsilon$ & The permittivity of the material with subscripts further \\
\hline & specifying its nature: $\varepsilon_{0}-$ free space and $\varepsilon_{\mathrm{r}}-$ relative for specific \\
\hline & material \\
\hline$\omega$ & Angular frequency \\
\hline $\overrightarrow{\mathrm{E}}, \overline{\mathrm{H}}$ & Elecrtric field vector and Magnetic field vector, respectively \\
\hline$\theta$ & Waveguide bend angle \\
\hline a & Lattice constant \\
\hline FDTD & Finite difference time domain \\
\hline PWM & Plane wave analysis \\
\hline 2-D & Two dimensional \\
\hline $\mathrm{T}$ & Transverse Magnetic \\
\hline
\end{tabular}


TE

$\Gamma$

$\mathrm{X}$

$\mathbf{M}$

$\vec{a}_{1}, \vec{a}_{2}$

$m_{1}, m_{2}$

$\vec{b}_{1}, \vec{b}_{2}$

$\bar{X} \bar{Y}$

$\varepsilon_{\mathrm{Al}}$

$\varepsilon_{\text {air }}$

$\overrightarrow{\mathbf{G}}$

$\mathbf{f}_{\mathbf{f}}$

$\mathbf{r}$

PML
Transverse Electric

When labeled on the band diagrams this is a point of symmetry in the reciprocal lattice.

When labeled on the band diagrams this is a point of symmetry in the reciprocal lattice.

When labeled on the band diagrams this is a point of symmetry in the reciprocal lattice.

Primitive translation vectors of the lattice

Integer numbers

Reciprocal lattice vectors

Periodicity plane

Dielectric constant of alumina rod

Dielectric constant of air

Reciprocal lattice vector

Filing factor

Alumina rod radius

Perfectly matched layer 
VNA

$S_{11}, S_{12}, S_{22}, S_{21}$

GPIB

$\mathbf{s}$

W

$\mathrm{dB}$

$\mathrm{GHz}$

$\mu \mathrm{m}$

$\mathrm{dBi}$
Vector network analyser

Scattering parameters

General purpose interface bus

Scaling factor

Waveguide channel width

Decibel

GigaHetz

Micrometer

Decibel "Isotropic" 


\section{Introduction}

In the last few decades, a growing demand for the development of fast, efficient circuits has become the leading driver in the integrated circuit industry. There are many advantages for designing switching elements using optical components, such as a decreased switching time, less cross-talk, increased reliability, increased fault tolerance, flexible service provisioning, etc. However, this industry has recently begun to slow down, as research has been restricted due to two primary problems:

i. Miniaturization of optic circuits resulting in an increase of resistance, ultimately leading to a high level of power dissipation.

ii. High speed and sensitivity for signal synchronization between different circuit components.

To overcome these problems, various scientists and scholars ${ }^{5-9}$ have begun to focus on the flow of light and on finding a suitable artificial periodic structure (photonic crystal) to cultivate photons efficiently at the microscale or nanoscale.

In 1987 , two research papers ${ }^{5-6}$ were published that determined the basic idea of electromagnetic modes at optical frequencies in multidimensional (1-D, 2-D, and 3-D) artificial periodic structures known as photonic crystals (as shown in Figure 1.1). A photonic crystal can be a low-loss periodic dielectric medium that prevents the flow of light in a certain direction for specified frequencies and can be useful in various communication applications. A 1-D dielectric structure is easy to fabricate and can even have an analytical solution with a photonic bandgap (PBG) for propagation along the direction of periodicity. A 2-D dielectric structure has PBG for all directions of 
propagation in a plane of periodicity that is uniform and can extend infinitely in the normal direction. A 3-D dielectric structure has full PBG for all possible directions of wave propagation, but a numerical analysis of such structures requires considerable time and computational capacity.
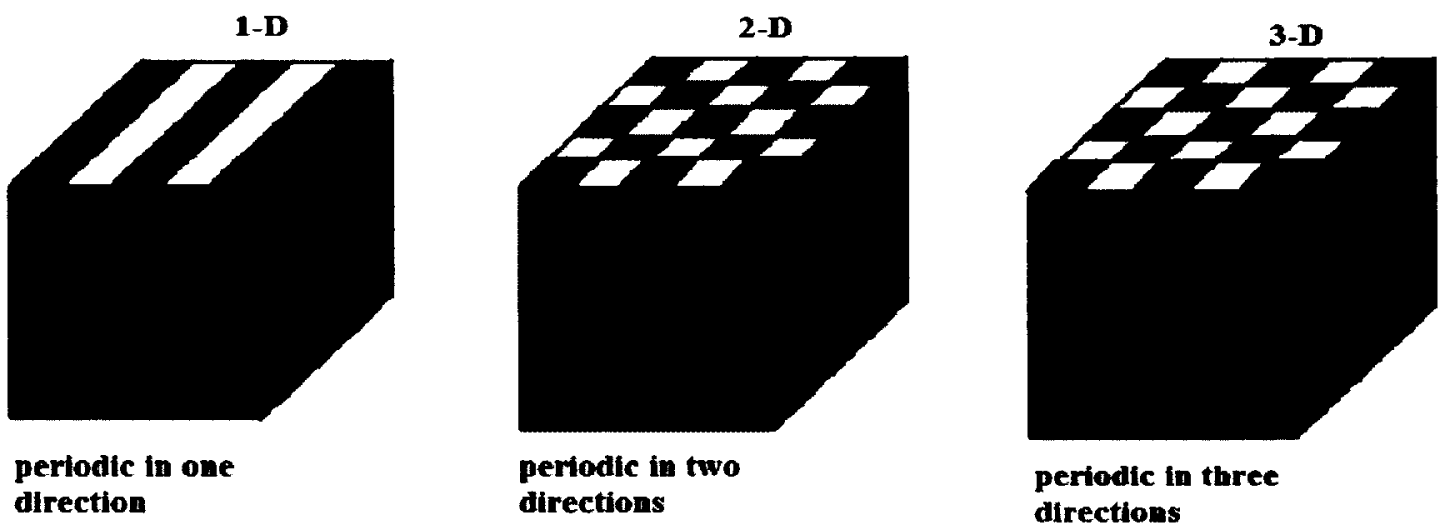

Figure 1.1: Schematic representation of multidimensional (1-D, 2-D, and 3-D) photonic crystals showing the periodicity of photonic crystals in one or more axes. The different colors demonstrate that a photonic crystal contains different materials with different dielectric constants. ${ }^{1}$

In order to design a photonic crystal with specific band behaviour, some key parameters must first be engineered, such as dimensionality, symmetry, topology, lattice parameter, filling factor, refractive index, and scalability. These parameters were determined with the help of Maxwell's equations to examine the behaviour of the photonic crystal. These equations were then solved for multidimensional (1-D, 2-D, and 3-D) ${ }^{9-13,15,17,18}$ dielectric structures, such as face-centered cubic $^{8,18}$ (primitive lattice) vectors (a collection of all points in space reachable from the origin with position vectors but are not orthogonal), a wood pile (an arrangement of dielectric materials using layer- 
by-layer stacking techniques such that layers are perpendicular to the layer below and then repeating the process until the structure is of desired height), and a self-organized structure $^{10}$ with different lattice patterns $s^{15,17,19,20-21,25-30,32,35-38,41,44-46}$ (square, triangular, and honeycomb, or combination of any two as heterostructures) by various computational methods (e.g., the plane wave method, ${ }^{13,19-21,30,32,36,69,71,77}$ the transfer matrix method, ${ }^{22,65}$ the finite difference time domain [FDTD], ${ }^{3-4,31,33,34-37,40,56,64,68,70-76}$ or the Korringa-KohnRostoker method ${ }^{23}$ ).

For the specific dielectric structure under observation, the concept of the Brillouin zone, reciprocal space, dispersion relation, and Bloch wave function were investigated to develop a photonic band structure. The photonic band structure represents the available energy state as a function of propagation direction. It shows a region (bandgap) where a certain frequency range is forbidden for a specified direction and polarization. It gives us information about the propagation properties of electromagnetic radiation within the photonic crystal.

$\mathrm{John}^{6}$ presented an observation for a 3-D disordered dielectric superlattice structure with a high dielectric constant showing strong localization of a photon at a certain frequency interval. Another 3-D dielectric structure ${ }^{7}$ consisting of an alumina rod ( $\varepsilon_{\mathrm{r}}=9.6$, where $\varepsilon_{\mathrm{r}}$ is a relative permittivity of the alumina rod) $0.32 \pm 0.01 \mathrm{~cm}$ in diameter and $15.24 \mathrm{~cm}$ long and a lattice constant of $\mathrm{a}=1.123 \mathrm{~cm}$ was fabricated and tested in the microwave regime to obtain a complete photonic bandgap between $11.7 \mathrm{GHz}$ and 13.5 GHz.

In 1991, Yablonovitch ${ }^{8-9}$ presented two research papers on a 3-D dielectric structure showing a complete photonic bandgap between $13 \mathrm{GHz}$ and $16 \mathrm{GHz}$ for both 
TM and TE polarization. ${ }^{1}$ The TM polarization was characterized with the electric field component $\left(E_{z}\right)$ parallel to the $\mathrm{z}$ direction and the magnetic field component $\left(H_{x}, H_{y}\right)$ in the $(x, y)$ plane as shown in Figure 1.2(a). TE polarization was characterized with the magnetic field component $\left(\mathrm{H}_{\mathrm{z}}\right)$ parallel to the $\mathrm{z}$ direction and the electric field component $\left(E_{x}, E_{y}\right)$ in the $(\mathrm{x}, \mathrm{y})$ plane as seen in Figure 1.2(b).

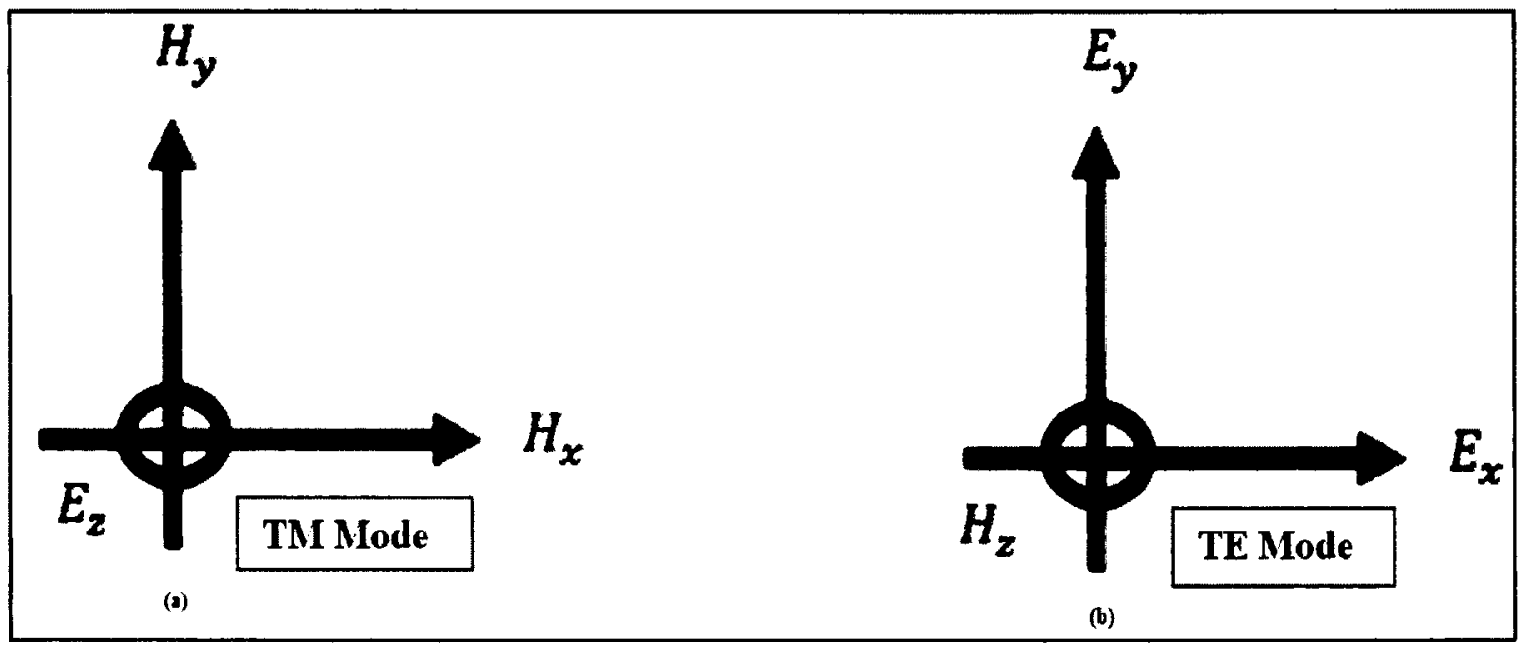

Figure 1.2: TM Polarization (left side): the magnetic field component $\left(H_{x}, H_{y}\right)$ confined to $(\mathrm{x}, \mathrm{y})$ plane and TE Polarization (right side): the electric field component $\left(E_{x}, E_{y}\right)$ confined to $(\mathrm{x}, \mathrm{y})$ plane.

Once a photonic band structure is investigated for a photonic crystal, photons can be controlled and guided within the photonic crystal in a particular direction with the introduction of defects that are either localized or extended, which causes an interruption in the periodicity. A defect design in the photonic crystal could be of any size, shape, or form and can be formed in a wide range of mediums with different dielectric constants. In 1994, Meade ${ }^{59}$ introduced the concept of microcavities and a linear waveguide to 
transmit optical signals between lasers and detectors on an optoelectronic integrated circuit. These two types of defects can be created within the photonic crystal in two ways:

i. By making a cavity in the center of the photonic crystal that forms an optical cage to trap photons, thereby acting like a resonator known as a point defect ${ }^{1-2,14,20,28-}$ 29,31,33-34,59 (a micro-cavity) as shown in Figure 1.3(a),

ii. By carving a tunnel (waveguide channel) through the dielectric material that forms an optical wire to guide the flow of light without scattering, which is known as an extended defect (a line defect ${ }^{1-2,14,16,20,26,33,59,60-61,72,75,77}$ ) as shown in Figure 1.3(b).

If the dielectric is removed to form a defect, then the cavity mode evolves from the dielectric band. Basically, the dielectric band is a low-frequency band where the energy of their modes is concentrated in a high dielectric region. Similarly, if the defect involves the addition of the extra dielectric medium, the cavity mode drops from the air band. The air band is a high-frequency band where the energy of their modes is concentrated in a low dielectric region. 


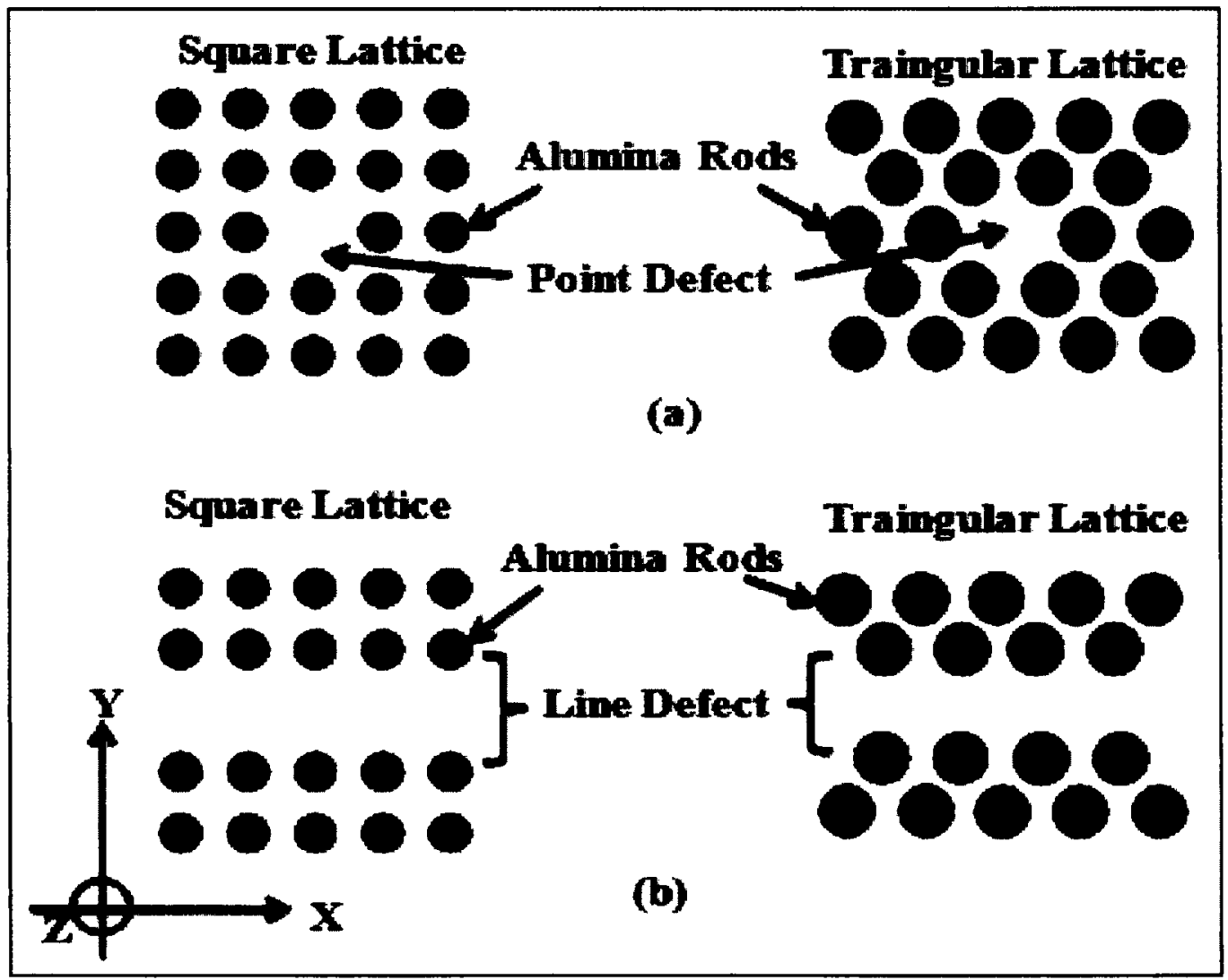

Figure 1.3: Defects in 2-D photonic crystal (square and triangular lattice), (a) a cavity (point) defect (b) an extended (line) defect.

For this thesis, the main work was carried out on a 2-D photonic crystal with a line defect that formed a linear waveguide. ${ }^{14,16,20,26,33,59-61,72,75,77}$ It guided an electromagnetic wave from one location to another through a specified channel (path). Figure 1.3(b) shows a 2-D photonic crystal (square and triangular lattice) linear waveguide formed by removing one or several rows of patterns along one of the directions of the crystal while maintaining their translation symmetry. A triangular lattice possesses large TE and TM gaps that open at a lower filling factor (the ratio of the crosssectional area of the dielectric column to the area of the primitive unit cell of the lattice) 
as compared to a square lattice. Light that propagated in a linear waveguide with a frequency within the photonic band gap was confined to the defect and could be directed along the waveguide channel of air or low dielectric constant. Basically, a linear waveguide must satisfy three criteria in order to achieve optimal performance in many integrated optical circuit applications, such as for maximum transmission through a sharp bend $^{60}$ and resonant cavities ${ }^{33}$ :

i. The waveguide structure must be periodic along the direction of propagation to achieve the well-defined wave number $k$ and thereby propagate without significant reflection.

ii. The waveguide should be single mode in the frequency range of interest.

iii. The guided mode should lie within the photonic band gap of a photonic crystal in order to minimize radiation losses.

The linear waveguides made with an extremely sharp low loss bend operating at 1.5 $\mu \mathrm{m}$ is of significant practical importance for the miniaturization of optoelectronic components and circuits ( $\mathrm{Y}$ branch, $\mathrm{T}$ branch, bends at different angles to form lossless waveguides, power splitter and combiners, Mach Zehnder). ${ }^{1-2,16,26-28,33,34,36-41,43-46}$

In 1996, Mekis $^{60}$ studied a 2-D photonic crystal of dielectric rods (GaAs) in the air and formed a single sharp bend by removing one row in a square array of infinitely long dielectric rods as shown in Figure 1.4. This structure provided $100 \%$ transmission efficiency through the waveguide with a sharp bend corner. 


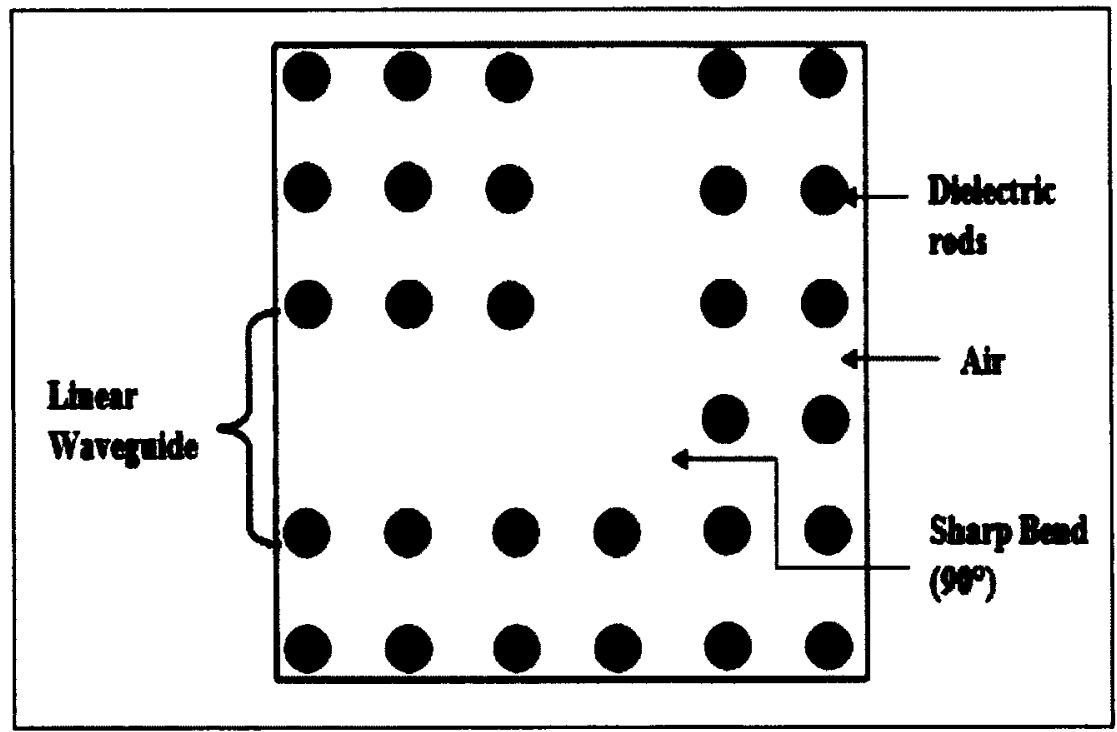

Figure 1.4: A 2-D photonic crystal (square lattice) linear waveguide with a sharp bend corner $\left(90^{\circ}\right)$.

$\operatorname{Lin}^{41}$ experimentally verified the above results in the microwave regime by using the same structure but different dielectric material (e.g., alumina rods). Multidimensional (2-D and 3-D) photonic crystal linear waveguide structures with different lattice patterns (square, triangular, or honeycomb) with different single bend angles $(60,90$, and 120 degrees) were studied theoretically and experimentally both in the optical and microwave regimes by various researchers, ${ }^{27,37-40,42,47,59,68,70-74,76,78}$ showing high transmission efficiency and reduced radiation losses.

After successful demonstration of a linear waveguide with a single bend at a different bend angle, both theoretically and experimentally, a double-bend angle ( $T, L$, and $U$ shapes) approach was introduced and studied with different lattice patterns. $^{26,36,55,62,64-65,69,72-73}$ Figure 1.5 shows a linear waveguide with a double bend ${ }^{26}$ consisting of dielectric rods in air with a square lattice with a low bend angle with TE polarization. In this structure, two bends were introduced along the waveguide where 
input and output waveguide segments were maintained parallel to each other. This structure provides low waveguide loss that finds a potential application in various optoelectronic circuit devices such as Mach Zehnder. ${ }^{26}$ The double-bend angle technique has led to the construction of waveguide branches ( $\mathrm{T}$ junction, $\mathrm{Y}$ branch) that have found wide application in the optical industry.

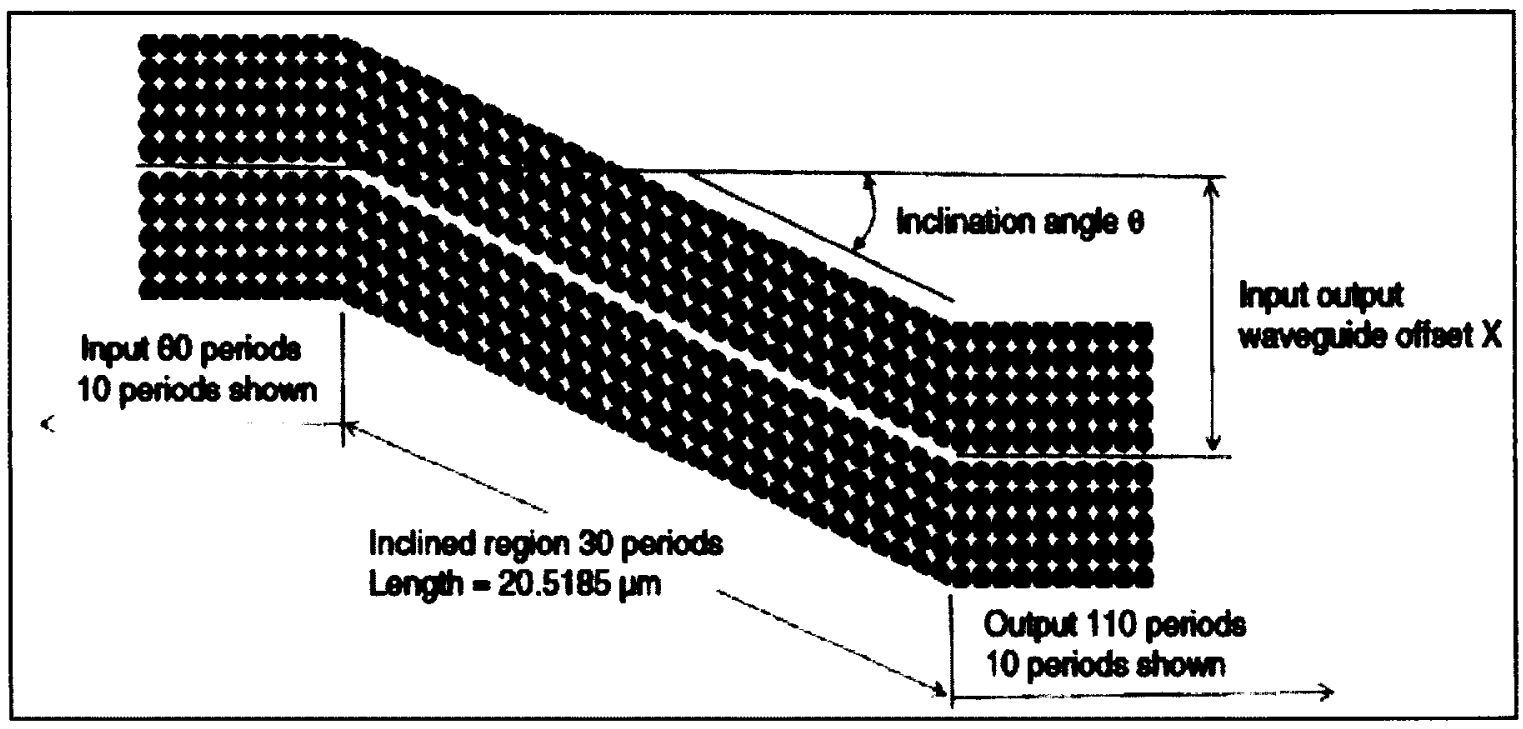

Figure 1.5: A linear waveguide ${ }^{26}$ with double bend where input and output waveguide segments are maintained parallel with an inclined waveguide at angle $(\theta)$ varied from $0^{\circ}$ to $20^{\circ}$.

Various research studies ${ }^{44-46,63-67}$ have been conducted to fabricate high-efficiency $\mathrm{Y}$ and $\mathrm{T}$ branches with large branch (split) angles (60 and 90 degrees each) as shown in Figure 1.6. 


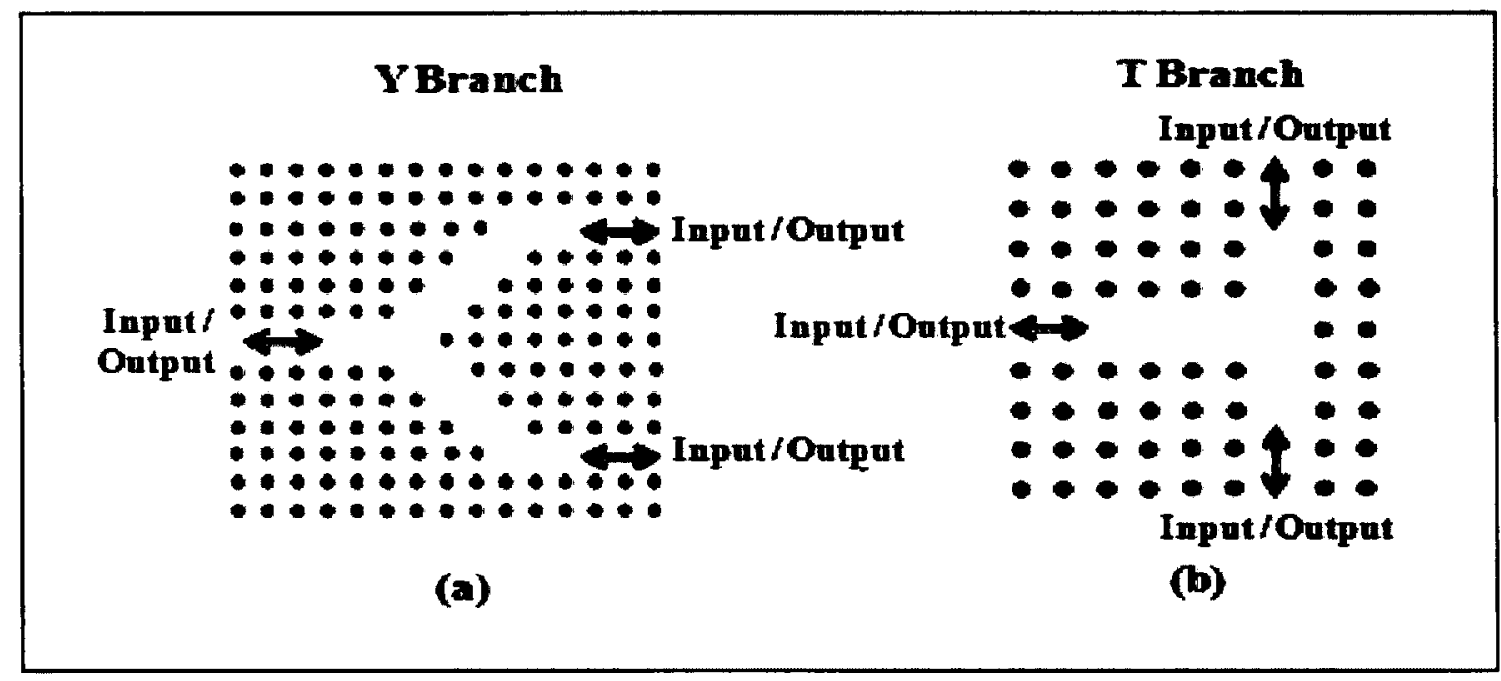

Figure 1.6: A 2-D photonic crystal linear waveguide with a square lattice, (a) $\mathrm{Y}$ branch, (b) $\mathrm{T}$ branch.

These waveguide branches ( $\mathrm{Y}$ and $\mathrm{T}$ ) are used for combining signals from two separate channels, for transmission of the combined signals over a single optical fibre link, and for separation of the individual channels at the receiver. But as these waveguide branches are studied with a large branch angle (split angle) but are impractical to build in the microwave regime. This main factor provides a strong motivation for designing, developing, and fabricating a Y-branch splitter with a small branch angle and high output efficiency.

\subsection{Motivation}

In 2012, Gauthier ${ }^{26}$ demonstrated FDTD-based simulation results for a 2-D photonic crystal waveguide with low loss bends presented between input and output. In addition, a $\mathrm{Y}$ branch with a square lattice for the TE polarization was proposed. The experimental verification of the simulated performance was one of the objectives of this thesis. 
Another research paper ${ }^{55}$ shows a photonic crystal waveguide structure examined theoretically and experimentally for a 2-D 8-, 10-, and 12-fold quasicrystal waveguide at different bend angles between input and output for optical (TE and TM) polarizations. These two studies show a direction for working with a 2-D photonic crystal linear waveguide with a triangular lattice at different waveguide bend angles $(\theta)$ and for designing and fabricating a 2-D Y-branch splitter with low transmission loss.

Note: The structures designed and fabricated both in the optical and regimes are referred to as "photonic crystals" throughout this thesis.

\subsection{Work Performed}

In this thesis, the following work contributes toward the fulfillment of motivation goal in the following ways:

i. Design and fabricate a template of a linear waveguide both theoretically and experimentally to obtain desired photonic band gaps and guided modes at microwave frequencies

ii. Analyze the theoretical and experimental results for a double-bend linear waveguide at different waveguide bend angles, $(\theta)$ varying from 0 to 30 degrees, and determine an optimal angle range that shows low transmission loss

iii. Fabricate a 2-D photonic crystal $Y$ splitter with a triangular lattice (TM polarization) in the microwave regime at the optimal waveguide bend angle $(\theta)$ 
with minimum transmission loss for a single wavelength that is guided through two output ports

The work for this thesis started by designing a 2-D small template using Styrofoam material both for a square and triangular lattice. This structure accommodates a 100 alumina rod. Various values for the lattice constant were selected to find a desirable photonic band gap in the frequency range ( $2 \mathrm{GHz}$ to $14 \mathrm{GHz}$ ). After choosing an optimal lattice constant and other design parameters, a 2-D photonic crystal double-bend linear waveguide with a triangular lattice was designed and fabricated using a plexi-glass sheet in the microwave regime. Both theoretical (optical) and experimental (microwave) measurements were conducted for a linear waveguide at different waveguide bend angles ( $\theta)$ at a particular frequency. After analyzing both theoretical and experimental results for a linear waveguide, a Y-splitter template was designed and fabricated at a small branch (split) angle ( $\theta)$ to obtain maximum efficiency for a single frequency (wavelength) at two output ports.

The software tools used in this thesis work were preexisting. These tools were used to plot photonic band structures using a plane wave method and transmission spectrum using the FDTD method.

\subsection{Contribution}

The main contribution was to design and fabricate a 2-D photonic crystal linear waveguide template with a triangular lattice in the microwave regime. This waveguide was built with plexi-glass material that accommodated 300 alumina rods. A layout 
diagram for a linear waveguide with all dimensions was created manually and followed while fabricating the structure at the machine shop. Further, a Y-branch splitter was fabricated by readjusting the design parameters of the linear waveguide template.

\subsection{Thesis Outline}

To achieve the objective of the study, a 2-D photonic crystal with a triangular lattice, small angle, low-loss $\mathrm{Y}$ splitter was designed and fabricated in the microwave regime. This whole procedure is discussed in the six chapters of the thesis. In Chapter 1, the concept of photonic crystal is briefly discussed, along with potential applications and the contribution of work done. Chapter 2 elaborates the theoretical framework, including Maxwell equations and the scalability concept implemented while covering various parameters from the optical regime to the microwave regime. In Chapter 3, various computational methods, such as a plane wave method and the FDTD method, along with simulation results, are presented. Chapter 4 demonstrates the experimental setup (design and fabrication) of a 2-D photonic crystal linear waveguide template using plexi-glass and the microwave experimental results for the linear waveguide. In Chapter 5, the formation of a double-bend waveguide is described using a dielectric shift technique at different waveguide bend angles. In addition, the design of a Y-branch splitter with a small branch angle is demonstrated in the microwave regime. Chapter 6 provides a conclusion of the thesis and indicates future directions for research. 


\section{Theoretical Framework}

A brief background concept of the photonic crystal with multidimensional structures was presented in the previous chapter. In this chapter, Maxwell's equations are recast in wave equations and provide access to explain the behaviour of photons within the multidimensional (2-D, 3-D) dielectric medium of interest. Further, for 2-D structures, these wave equations were developed for two optical polarizations (TM and TE), and a scalability law was implemented for the TM polarization to change design parameters from an optical to a microwave regime without changing the physical properties of the structure.

A 2-D linear waveguide structure was designed in an optical and a microwave regime and implemented in the microwave regime by placing alumina rods in the air. Numerical methods, such as a plane wave method (PWM) and finite difference time domain (FDTD) were used to theoretically analyze the structure. These methods provide photonic band structures, bandgap maps and transmission spectra that help to analyze the 2-D linear waveguide structure. All theoretical results were obtained with the help of these numerical methods and were verified experimentally by designing and fabricating a 2-D linear waveguide structure for the microwave regime. This leads to the fabrication of a $\mathrm{Y}$ branch splitter in the microwave regime.

\subsection{Maxwell's Equations}

In a photonic crystal, electromagnetic waves are solved with the help of Maxwell's equations. The macroscopic Maxwell equations ${ }^{1}$ are: 


$$
\begin{gathered}
\vec{\nabla} \cdot \vec{D}=\rho \\
\vec{\nabla} \times \vec{H}-\frac{\partial \vec{D}}{\partial t}=\vec{\jmath} \\
\vec{\nabla} \cdot \vec{B}=0 \\
\vec{\nabla} \times \overrightarrow{\mathrm{E}}+\frac{\partial \overrightarrow{\mathrm{B}}}{\partial \mathrm{t}}=0
\end{gathered}
$$

Where, $\overrightarrow{\mathrm{D}}$ and $\overrightarrow{\mathrm{B}}$ are the electric flux and the magnetic induction and $\rho$, as free charge and $\vec{\jmath}$, as current.

In the case of a homogeneous dielectric medium, the relationship between the macroscopic quantities, that is the electric flux $\vec{D}$ and the magnetic induction $\vec{B}$, and the microscopic quantities $\overrightarrow{\mathrm{E}}$ and $\overrightarrow{\mathrm{H}}$ are given by $\overrightarrow{\mathrm{D}}=\varepsilon_{r} \varepsilon_{0} \overrightarrow{\mathrm{E}}$, where $\varepsilon_{r}$ is relative permittivity and $\varepsilon_{0}=8.544187817 \times 10^{-12} \mathrm{~F} / \mathrm{m}$ is permittivity in free space, and, $\overrightarrow{\mathrm{B}}=\mu_{0} \mu_{\mathrm{r}} \overrightarrow{\mathrm{H}}$, where $\mu_{0}=4 \pi \times 10^{-7} \mathrm{H} / \mathrm{m}$ is the vacuum permeability and $\mu_{r} \approx 1$ is the relative magnetic permeability for non-magnetic materials.

For a photonic crystal structure, the following assumptions ${ }^{1}$ were made to solve the eigenvalue equations for band structure computation:

i. Free charges $(\rho)$ and current $(j)$ are neglected.

ii. The field strengths are small enough to be in the linear regime. 
iii. The material is locally macroscopic and isotropic, i.e. dielectric constant ' $\varepsilon(r)$ ' is a scalar.

iv. Only lossless dielectrics are considered for which " $\varepsilon(r)$ " is a real quantity. Considering the following above assumptions, equations (2-1) to (2-4) can be written as:

$$
\begin{gathered}
\vec{\nabla} \cdot \vec{H}(\vec{r}, t)=0 \\
\vec{\nabla} \cdot[\varepsilon(\vec{r}) \vec{E}(\vec{r}, t)]=0 \\
\vec{\nabla} \times \vec{E}(\vec{r}, t)+\mu_{0} \frac{\partial \vec{H}(\vec{r}, t)}{\partial t}=0 \\
\vec{\nabla} \times \vec{H}(\vec{r}, t)-\varepsilon(\vec{r}) \frac{\partial \vec{E}(\vec{r}, t)}{\partial t}=0
\end{gathered}
$$

To get a harmonic solution with a sinusoidal time dependence, the fields can be written in complex form as:

$$
\begin{aligned}
& \vec{E}(\vec{r}, t)=\vec{E}(\vec{r}) e^{j \omega t} \\
& \vec{H}(\vec{r}, t)=\vec{H}(\vec{r}) e^{j \omega t}
\end{aligned}
$$

Where $\omega=$ angular frequency $=2 \pi f ;(f=$ frequency $)$. 
If the time-dependent equations (2-10) and (2-11) are substituted in the equations (2-7) and (2-8), the modified form is obtained:

$$
\begin{aligned}
& \vec{\nabla} \times \overrightarrow{\mathrm{E}}(\overrightarrow{\mathrm{r}})=-j \omega \mu_{0} \overrightarrow{\mathrm{H}}(\overrightarrow{\mathrm{r}}) \\
& \vec{\nabla} \times \overrightarrow{\mathrm{H}}(\overrightarrow{\mathrm{r}})=j \omega \varepsilon(\overrightarrow{\mathrm{r}}) \overrightarrow{\mathrm{E}}(\overrightarrow{\mathrm{r}})
\end{aligned}
$$

For the 3-D structure, the equation for a magnetic field obtained by dividing equation (2-13) by $\varepsilon(\overrightarrow{\mathrm{r}})$, taking the curl $(\vec{\nabla} \times)$ from the left-hand side and using equation (2-12) to eliminate $\overrightarrow{\mathrm{E}}$, an eigenvalue (master) equation for magnetic field results:

$$
\left(\vec{\nabla} \times\left(\frac{1}{\varepsilon(\overrightarrow{\mathrm{r}})} \vec{\nabla} \times \overrightarrow{\mathrm{H}}(\overrightarrow{\mathrm{r}})\right)\right)=\left(\frac{\omega}{\mathrm{c}}\right)^{2} \overrightarrow{\mathrm{H}}(\overrightarrow{\mathrm{r}})
$$

For the electric field, the eigenvalue (master) equation is obtained by taking the curl $(\vec{\nabla} \times)$ from the left-hand side of the equation (2-12), using equation (2-13) and dividing by $\varepsilon(\overrightarrow{\mathrm{r}})$ to eliminate $\overrightarrow{\mathrm{H}}$ :

$$
\frac{1}{\varepsilon(\overrightarrow{\mathrm{r}})} \vec{\nabla} \times \vec{\nabla} \times \overrightarrow{\mathrm{E}}(\overrightarrow{\mathrm{r}})=\left(\frac{\omega}{\mathrm{c}}\right)^{2} \overrightarrow{\mathrm{E}}(\overrightarrow{\mathrm{r}})
$$


In the $(\mathrm{x}, \mathrm{y}, \mathrm{z})$ coordinate system, the electric field and magnetic fields are:

$$
\begin{aligned}
& \vec{E}=E_{x} \hat{x}+E_{y} \hat{y}+E_{z} \hat{z} \\
& \vec{H}=H_{x} \hat{x}+H_{y} \hat{y}+H_{z} \hat{z}
\end{aligned}
$$

Equations (2-7) and (2-16) become:

$$
\vec{\nabla} \times \vec{E}(\vec{r}, t)=\left|\begin{array}{ccc}
\hat{x} & \hat{y} & \hat{z} \\
\frac{\partial}{\partial x} & \frac{\partial}{\partial y} & \frac{\partial}{\partial z} \\
E_{x} & E_{y} & E_{z}
\end{array}\right|=\left(\frac{\partial E_{z}}{\partial y}-\frac{\partial E_{y}}{\partial z}\right) \hat{x}-\left(\frac{\partial E_{z}}{\partial x}-\frac{\partial E_{x}}{\partial z}\right) \hat{y}+\left(\frac{\partial E_{y}}{\partial x}-\frac{\partial E_{x}}{\partial y}\right) \hat{z}
$$

Inserting (2-18) into (2-7) gives a set of scalar equations relating the components of the electric and magnetic fields:

$$
\begin{aligned}
& \left(\frac{\partial \mathrm{E}_{\mathrm{z}}}{\partial \mathrm{y}}-\frac{\partial \mathrm{E}_{\mathrm{y}}}{\partial \mathrm{z}}\right)=-\mu_{0} \frac{\partial \mathrm{H}_{\mathrm{x}}}{\partial \mathrm{t}} \\
& \left(\frac{\partial \mathrm{E}_{\mathrm{z}}}{\partial \mathrm{x}}-\frac{\partial \mathrm{E}_{\mathrm{x}}}{\partial \mathrm{z}}\right)=-\mu_{0} \frac{\partial \mathrm{H}_{\mathrm{y}}}{\partial \mathrm{t}} \\
& \left(\frac{\partial \mathrm{E}_{\mathrm{y}}}{\partial \mathrm{x}}-\frac{\partial \mathrm{E}_{\mathrm{x}}}{\partial \mathrm{y}}\right)=-\mu_{0} \frac{\partial \mathrm{H}_{\mathrm{z}}}{\partial \mathrm{t}}
\end{aligned}
$$

The expansion of the curl in equation (2-8) making use of equation (2-17) gives: 


$$
\vec{\nabla} \times \vec{H}=\left|\begin{array}{ccc}
\hat{\mathbf{x}} & \hat{\mathrm{y}} & \hat{\mathbf{z}} \\
\frac{\partial}{\partial \mathrm{x}} & \frac{\partial}{\partial \mathrm{y}} & \frac{\partial}{\partial \mathrm{z}} \\
\mathrm{H}_{\mathrm{x}} & \mathrm{H}_{\mathrm{y}} & \mathrm{H}_{\mathrm{z}}
\end{array}\right|=\left(\frac{\partial \mathrm{H}_{\mathrm{z}}}{\partial \mathrm{y}}-\frac{\mathrm{H}_{\mathrm{y}}}{\partial \mathrm{z}}\right) \hat{\mathrm{x}}-\left(\frac{\partial \mathrm{H}_{\mathrm{z}}}{\partial \mathrm{x}}-\frac{\partial \mathrm{H}_{\mathrm{x}}}{\partial \mathrm{z}}\right) \hat{\mathrm{y}}+\left(\frac{\partial \mathrm{H}_{\mathrm{y}}}{\partial \mathrm{x}}-\frac{\partial \mathrm{H}_{\mathrm{x}}}{\partial \mathrm{y}}\right) \hat{\mathbf{z}}
$$

When inserted in equation (2-8) results in the following three expressions relating the field components:

$$
\begin{aligned}
& \left(\frac{\partial \mathrm{H}_{\mathrm{z}}}{\partial \mathrm{y}}-\frac{\partial \mathrm{H}_{\mathrm{y}}}{\partial \mathrm{z}}\right)=\varepsilon(\overrightarrow{\mathrm{r}}) \frac{\partial \mathrm{E}_{\mathrm{x}}}{\partial \mathrm{t}} \\
& \left(-\frac{\partial \mathrm{H}_{\mathrm{z}}}{\partial \mathrm{x}}-\frac{\partial \mathrm{H}_{\mathrm{x}}}{\partial \mathrm{z}}\right)=\varepsilon(\overrightarrow{\mathrm{r}}) \frac{\partial \mathrm{E}_{\mathrm{y}}}{\partial \mathrm{t}} \\
& \left(\frac{\partial \mathrm{H}_{\mathrm{y}}}{\partial \mathrm{x}}-\frac{\partial \mathrm{H}_{\mathrm{x}}}{\partial \mathrm{y}}\right)=\varepsilon(\overrightarrow{\mathrm{r}}) \frac{\partial \mathrm{E}_{\mathrm{z}}}{\partial \mathrm{t}}
\end{aligned}
$$

For a 2-D analysis in $(\mathrm{X}, \mathrm{Y})$ and uniform infinite in $\mathrm{Z}$-direction, the derivatives of the magnetic and electric field components with respect to ' $\mathrm{Z}$ ' can be set to zero, $\left[\frac{\partial}{\partial z}=0\right]$. Then the field components decouple into two polarization sets. The next section will investigate the 2-D photonic crystal for both polarizations.

\subsection{2-D Photonic Crystal Wave Equation for TM and TE Polarization}

The 2-D planar, uniform and infinite in the $\mathrm{Z}$ direction structures as shown in Figure 2.1. The left side shows the square array and the right side shows the triangular array with the lattice constant ' $a$ ' in the $(X, Y)$ plane. The gray circles represent a high dielectric 
region, such as the alumina rods, and the white regions represent another medium, such as air of the lower dielectric constant.

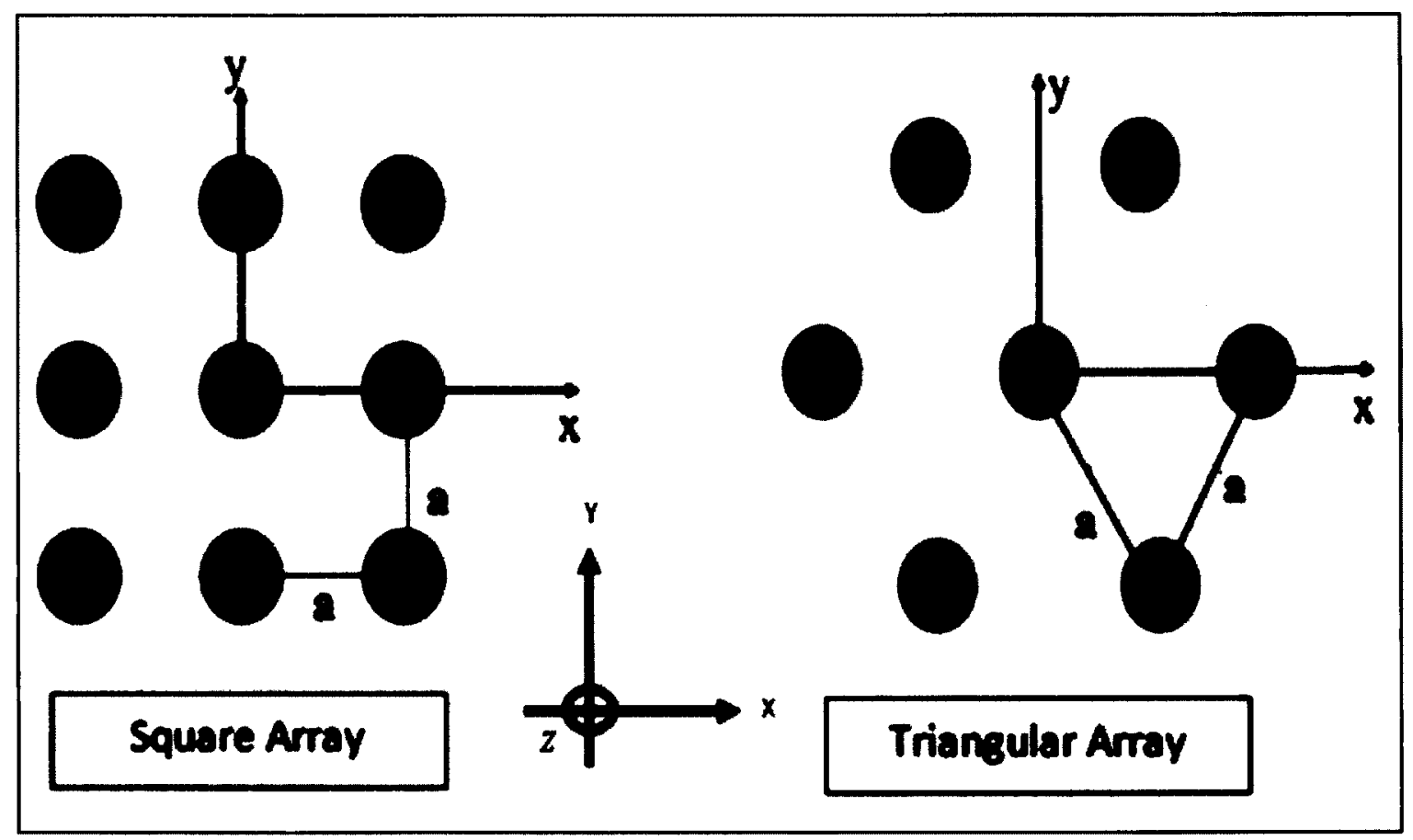

Figure 2.1: Square (left) and triangular (right) lattice photonic crystals. The gray circles represent alumina rods with relative dielectric constant; ' $\varepsilon_{\mathrm{Al}}$ ' and rod radius; ' $r$ '. The lattice constant is ' $a$ '. The space between rods in white represent air with relative dielectric constant; ' $\varepsilon_{\text {alr }}$ '.

The rod axis runs parallel to the $\mathrm{Z}$ coordinate axis. As indicated above, for this particular geometry, the six field components in equations (2-19), (2-20), (2-21), (2-23), (2-24) and (2-25) decouple into two distinct polarizations: either $\left(H_{x}, H_{y}, E_{z}\right)$ or $\left(E_{x}, E_{y}, H_{z}\right)$. The former, in which the magnetic field components $\left(H_{x}, H_{y}\right)$ are confined to the $(\mathrm{X}, \mathrm{Y})$ plane is called the transverse magnetic (TM) polarization'. The latter, in which the 
electric field components $\left(E_{x}, E_{y}\right)$ are confined to the $(\mathrm{X}, \mathrm{Y})$ plane is called the transverse electric (TE) polarization ${ }^{1}$.

\subsubsection{TM Polarization}

For TM polarization, the equations (2-20), (2-21) and (2-25) can be isolated from the original six equations and can be rewritten as:

$$
\begin{gathered}
\left(\frac{\partial \mathrm{E}_{\mathrm{z}}}{\partial \mathrm{y}}\right)=-\mu_{0} \frac{\partial \mathrm{H}_{\mathrm{x}}}{\partial \mathrm{t}} \\
\left(-\frac{\partial \mathrm{E}_{\mathrm{z}}}{\partial \mathrm{x}}\right)=-\mu_{0} \frac{\partial \mathrm{H}_{\mathrm{y}}}{\partial \mathrm{t}} \\
\left(\frac{\partial \mathrm{H}_{\mathrm{y}}}{\partial \mathrm{x}}-\frac{\partial \mathrm{H}_{\mathrm{x}}}{\partial \mathrm{y}}\right)=\varepsilon(\overrightarrow{\mathrm{r}}) \frac{\partial \mathrm{E}_{\mathrm{z}}}{\partial \mathrm{t}}
\end{gathered}
$$

Taking the time derivative of equation (2-28) gives:

$$
\left(\frac{\partial}{\partial \mathrm{t}} \frac{\partial \mathrm{H}_{\mathrm{y}}}{\partial \mathrm{x}}-\frac{\partial}{\partial \mathrm{t}} \frac{\partial \mathrm{H}_{\mathrm{x}}}{\partial \mathrm{y}}\right)=\varepsilon(\overrightarrow{\mathrm{r}}) \frac{\partial^{2} \mathrm{E}_{\mathrm{z}}}{\partial \mathrm{t}^{2}}
$$

Taking the y derivative of equation (2-26): 


$$
\left(\frac{\partial^{2} E_{z}}{\partial y^{2}}\right)=-\mu_{0} \frac{\partial}{\partial y} \frac{\partial H_{x}}{\partial t}
$$

Taking the $\mathrm{x}$ derivative of equation (2-27):

$$
\left(-\frac{\partial^{2} E_{z}}{\partial x^{2}}\right)=-\mu_{0} \frac{\partial}{\partial x} \frac{\partial H_{y}}{\partial t}
$$

By combining equations (2-29), (2-30) and (2-31), gives the scalar wave equation for the z-component of the electric field as:

$$
\frac{1}{\varepsilon(\vec{r})}\left(\frac{\partial^{2} E_{z}}{\partial x^{2}}+\frac{\partial^{2} E_{z}}{\partial y^{2}}\right)=\frac{1}{c^{2}} \frac{\partial^{2} E_{z}}{\partial t^{2}}
$$

Further, evaluating derivatives with respect to time, gives:

$$
\frac{1}{\varepsilon(\vec{r})}\left(\frac{\partial^{2}}{\partial x^{2}}+\frac{\partial^{2}}{\partial y^{2}}\right) E_{z}+\frac{\omega^{2}}{c^{2}} E_{z}=0
$$

Once the electric field component $\left(E_{z}\right)$ is solved using the equation (2-33), the magnetic field components $\left(\mathrm{H}_{\mathrm{x}}, \mathrm{H}_{\mathrm{y}}\right)$ can be obtained using equations (2-26) and (2-27). 


\subsubsection{TE Polarization}

For TE polarization, the equations (2-21), (2-23) and (2-24) can be isolated from the original six equations and rewritten as:

$$
\begin{gathered}
\left(\frac{\partial \mathrm{E}_{\mathrm{y}}}{\partial \mathrm{x}}-\frac{\mathrm{E}_{\mathrm{x}}}{\partial \mathrm{y}}\right)=-\mu_{0} \frac{\partial \mathrm{H}_{\mathrm{z}}}{\partial \mathrm{t}} \\
\left(\frac{\partial \mathrm{H}_{\mathrm{z}}}{\partial \mathrm{y}}\right)=\varepsilon(\overrightarrow{\mathrm{r}}) \frac{\partial \mathrm{E}_{\mathrm{x}}}{\partial \mathrm{t}} \\
\left(-\frac{\partial \mathrm{H}_{\mathrm{z}}}{\partial \mathrm{x}}\right)=\varepsilon(\overrightarrow{\mathrm{r}}) \frac{\partial \mathrm{E}_{\mathrm{y}}}{\partial \mathrm{t}}
\end{gathered}
$$

Equations (2-35) and (2-36) can be written as:

$$
\begin{gathered}
\frac{1}{\varepsilon(\overrightarrow{\mathrm{r}})}\left(\frac{\partial \mathrm{H}_{\mathrm{z}}}{\partial \mathrm{y}}\right)=\frac{\partial \mathrm{E}_{\mathrm{x}}}{\partial \mathrm{t}} \\
\frac{1}{\varepsilon(\overrightarrow{\mathrm{r}})}\left(-\frac{\partial \mathrm{H}_{\mathrm{z}}}{\partial \mathrm{x}}\right)=\frac{\partial \mathrm{E}_{\mathrm{y}}}{\partial \mathrm{t}}
\end{gathered}
$$

Taking the time derivative of equation (2-34) gives:

$$
\left(\frac{\partial}{\partial t} \frac{\partial E_{y}}{\partial x}-\frac{\partial}{\partial t} \frac{E_{x}}{\partial y}\right)=-\mu_{0} \frac{\partial^{2} H_{z}}{\partial t^{2}}
$$


Taking the y derivative of equation (2-37):

$$
\frac{\partial}{\partial y}\left(\frac{1}{\varepsilon(\overrightarrow{\mathrm{r}})}\left(\frac{\partial \mathrm{H}_{\mathrm{z}}}{\partial \mathrm{y}}\right)\right)=\frac{\partial}{\partial \mathrm{y}} \frac{\partial \mathrm{E}_{\mathrm{x}}}{\partial \mathrm{t}}
$$

Taking the $x$ derivative of equation (2-38):

$$
\frac{\partial}{\partial x}\left(\frac{1}{\varepsilon(\vec{r})}\left(-\frac{\partial \mathrm{H}_{z}}{\partial \mathrm{x}}\right)\right)=\frac{\partial}{\partial \mathrm{x}} \frac{\partial \mathrm{E}_{\mathrm{y}}}{\partial \mathrm{t}}
$$

By combining equations (2-39), (2-40) and (2-41), the scalar wave equation for the zcomponent of the magnetic field $\left(\mathrm{H}_{\mathrm{z}}\right)$ is:

$$
\frac{1}{\varepsilon(\overrightarrow{\mathrm{r}})}\left(\frac{\partial^{2} \mathrm{H}_{\mathrm{z}}}{\partial \mathrm{x}^{2}}+\frac{\partial^{2} \mathrm{H}_{\mathrm{z}}}{\partial \mathrm{y}^{2}}\right)=\frac{1}{\mathrm{c}^{2}} \frac{\partial^{2} \mathrm{H}_{\mathrm{z}}}{\partial \mathrm{t}^{2}}
$$

Further, evaluating derivatives with respect to time, gives:

$$
\frac{\partial}{\partial x}\left[\frac{1}{\varepsilon(\vec{r})} \frac{\partial}{\partial x} H_{z}\right]+\frac{\partial}{\partial y}\left[\frac{1}{\varepsilon(\vec{r})} \frac{\partial}{\partial y} H_{z}\right]+\frac{\omega^{2}}{c^{2}} H_{z}=0
$$

Once the magnetic field component $\left(H_{z}\right)$ is found, the electric field components $\left(E_{x}, E_{y}\right)$ can be obtained using equations (2-35) and (2-36). 


\subsection{Scalability Law}

An interesting and important property of electromagnetics is their scalability as there are no fundamental constants with the dimensions of length. That is the Maxwell equations are scale invariant ${ }^{1}$. Through this property, photonic crystal structures can be designed in the optical domain, and models constructed and tested in the microwave regime, at the much larger scale of centimeters.

Recalling the 3-D master equation in the electric field as:

$$
\frac{1}{\varepsilon(\overrightarrow{\mathrm{r}})} \vec{\nabla} \times \vec{\nabla} \times \overrightarrow{\mathrm{E}}(\overrightarrow{\mathrm{r}})=\left(\frac{\omega}{c}\right)^{2} \overrightarrow{\mathrm{E}}(\overrightarrow{\mathrm{r}})
$$

The dielectric system is now scaled by the scaling parameter, such that $\vec{r}^{\prime}=s \vec{r}$ and $\vec{\nabla}^{\prime}=\nabla / \mathrm{s}$. Now, change the variables in the equation $(2-44)$ as:

$$
\frac{1}{\varepsilon\left(\vec{r}^{\prime} / s\right)} s \vec{\nabla}^{\prime} \times s \vec{\nabla}^{\prime} \times \vec{E}\left(\vec{r}^{\prime} / s\right)=\left(\frac{\omega}{c}\right)^{2} \vec{E}\left(\vec{r}^{\prime} / s\right)
$$

By defining $\varepsilon^{\prime}$ as the dielectric constant of the stretched dielectric system, $\varepsilon^{\prime}(\overrightarrow{\mathrm{r}})=\varepsilon\left(\overrightarrow{\mathrm{r}}^{\prime} / \mathrm{s}\right)$ and rewritten as:

$$
\frac{1}{\varepsilon^{\prime}(\overrightarrow{\mathrm{r}})} \nabla^{\prime} \times\left(\nabla^{\prime} \times \overrightarrow{\mathrm{E}}\left(\overrightarrow{\mathrm{r}}^{\prime} / \mathrm{s}\right)\right)=\left(\frac{\omega}{\mathrm{cs}}\right)^{2} \overrightarrow{\mathrm{E}}\left(\overrightarrow{\mathrm{r}}^{\prime} / \mathrm{s}\right)
$$


This equation is also a master equation for the new mode of the scaled system with its frequency given by $\omega^{\prime}=\omega / \mathrm{s}$.

In this thesis, experimental and simulation work was carried out for a 2-D photonic crystal structure with a triangular lattice in TM polarization with a chosen lattice constant, (a) $\mu \mathrm{m}$, from the bandgap map. By inserting a conversion factor, $\mathrm{s}$, the lattice constant transforms with an enlarged lattice constant, $\left(a_{1}\right) \mathrm{cm}$.

For the 2-D structure, the master equation for the electric field is given as:

$$
\frac{1}{\varepsilon(\overrightarrow{\mathrm{r}})}\left(\frac{\partial^{2}}{\partial \mathrm{x}^{2}}+\frac{\partial^{2}}{\partial \mathrm{y}^{2}}\right) \overrightarrow{\mathrm{E}_{\mathrm{z}}}+\frac{\omega^{2}}{c^{2}} \overrightarrow{\mathrm{E}_{\mathrm{z}}}=0
$$

Following similar steps as in the 3-D structure for rescaling the 2-D structure, gets the equation:

$$
\frac{1}{\varepsilon\left(\overrightarrow{\mathrm{r}}^{\prime} / \mathrm{s}\right)}\left(\frac{\partial^{2}}{\partial \mathrm{x}^{2}}+\frac{\partial^{2}}{\partial \mathrm{y}^{2}}\right) \overrightarrow{\mathrm{E}_{\mathrm{z}}}\left(\overrightarrow{\mathrm{r}}^{\prime} / \mathrm{s}\right)+\left(\frac{\omega}{c s}\right)^{2} \overrightarrow{\mathrm{E}_{\mathrm{z}}}\left(\overrightarrow{\mathrm{r}}^{\prime} / \mathrm{s}\right)=0
$$

Therefore, once the properties of a photonic crystal for one lattice constant are calculated, then the whole structure can be rescaled to get the same features at the desired angular frequency, $\omega$. So, to calculate a new lattice constant, $a_{1}$, the following expressions are used:

$$
\omega_{2}=s \omega_{1}
$$




$$
\begin{gathered}
s a_{1}=a \\
\omega_{1} a=\omega_{2} a_{1} \\
\frac{\omega_{1} a}{\omega_{2}}=a_{1}
\end{gathered}
$$

The next chapter will explain the concept of various numerical methods (plane wave method and FDTD method). These methods will help to design the 2-D photonic crystal in the optical regime. Various simulation results are presented and verified experimentally in the following chapters. 


\section{Computational Methods}

In order to simulate a photonic crystal in any dimensional periodicity, different commercial and readily available simulation tools are used. These tools are extremely well known and already described in the various textbooks ${ }^{1,2,3,4}$. Simulation methods, such as Green's function expansion method ${ }^{23,51,52}$, the plane wave method $^{10,11,13,15,17,19,20,29,32,37}$, and the finite difference time domain method ${ }^{26,31,33,34,38}$, are widely used in the study of photonic crystal structures with their own advantages and limitations. Only the plane wave and finite difference time domain methods were used for simulation purposes. The simulation programs developed by my supervisor and I have already been used by many Master's and PhD students in their research work ${ }^{26,31,50}$.

\subsection{Plane Wave Method: Two-Dimensional Photonic Crystal}

To obtain photonic band structures ${ }^{11,18}$, the plane wave method was first implemented on three-dimensional periodic dielectric structures. This method is an adaptation of a classical method and takes advantage of the periodicity of the lattice to calculate band structures in photonic crystals. A two-dimensional photonic crystal is considered for plane wave analysis as all the work throughout the thesis is carried out on this type of structure.

The most significant fact about two-dimensional photonic crystals is that they can control the flow of light in two directions. The eigenvalue calculations in twodimensional structures are much easier than three-dimensional photonic crystals. Due to 
these advantages, they can easily be fabricated to any scale (optical as well as microwave regime). In 1991, the existence of a photonic bandgap in the simple square and triangular lattice two-dimensional photonic crystal was depicted ${ }^{19,21}$. Both (square and triangular) lattices consist of an array of infinitely long dielectric (alumina) rods with a circular cross-section of radius, ' $r$ '. The dielectric columns (alumina rods) of dielectric constant ' $\varepsilon_{\mathrm{Al}}$ ' are assumed to be parallel to the $\mathrm{z}$ axis. The intersections of the axes of these rods with the $(x, y)$ plane form a two-dimensional (square or triangular) lattice with lattice constant ' $\mathrm{a}$ '. The two-dimensional structure is defined through the set of lattice vectors $\vec{R}$ that can be generated by:

$$
\overrightarrow{\mathbf{R}}(\mathbf{m})=\mathbf{m}_{\mathbf{1}} \overrightarrow{\mathbf{a}}_{\mathbf{1}}+\mathbf{m}_{\mathbf{2}} \overrightarrow{\mathbf{a}}_{\mathbf{2}}
$$

where $\vec{a}_{1}, \vec{a}_{2}$ are the primitive translation vectors of the lattice, and $\mathrm{m}_{1}, \mathrm{~m}_{2}$ are any two integers.

Figure 3.1 (a) and (b) represents the primitive lattice vector and reciprocal space of a 2-D photonic crystal with a square lattice of columns of a high dielectric constant, ' $\varepsilon_{\mathrm{Al}}$ ' (black dots) surrounded in a background of low dielectric constant, ' $\varepsilon_{\text {air }}$ ' (white region). 


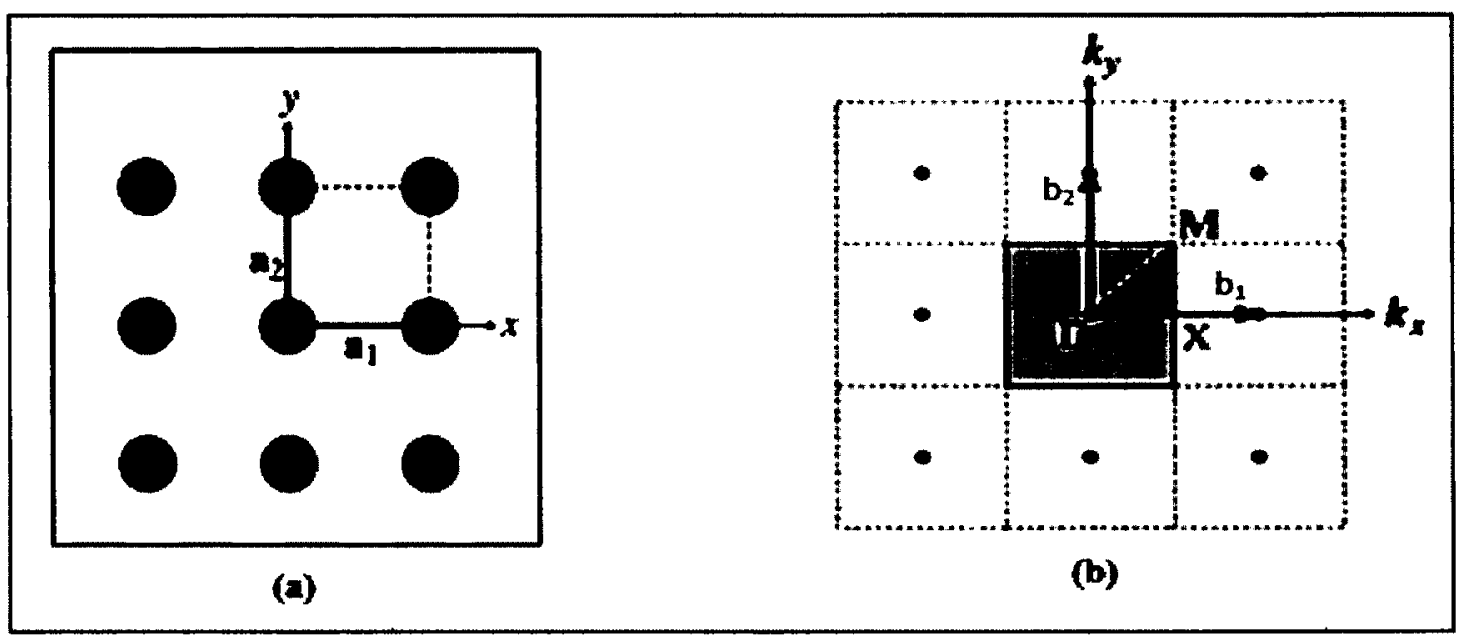

Figure 3.1: A 2-D photonic crystal with square lattice of columns of high dielectric constant, ' $\varepsilon_{A}$ ' (black dots) surrounded in a background of low dielectric constant, ' $\varepsilon_{\text {air }}$ ' (white region) (a) real space arrangement with two primitive lattice vectors $\vec{a}_{1}$, and $\vec{a}_{2} ;$ (b) reciprocal space with two reciprocal lattice vectors $\vec{b}_{1}$ and $\vec{b}_{2}$; the Brillouin zone is outlined by a solid bold border and the black shaded triangular region with points $\Gamma X M$ represents the irreducible $1 / 8$ Brillouin zone.

The area of the primitive unit cell of the lattice equal to $A_{u n i t}=\left|\vec{a}_{1} \times \vec{a}_{2}\right|$. A unit cell is a spatial domain (photonic crystal lattice) that is defined by its shape and the permittivity or refractive index distribution inside the cell.

With the substitution of all possible integer values in equation (3-1), a Bravais lattice was generated that consists of an infinite number of points periodically distributed through the plane. The $\vec{R}$ vectors thus obtained are also called a primitive lattice. The reciprocal lattice is formed by the set of vector $\vec{G}$ such that:

$$
\overrightarrow{\mathbf{G}} \cdot \overrightarrow{\mathbf{R}}=\mathbf{n} \mathbf{2} \boldsymbol{\pi}
$$

where $\mathrm{n}=$ integer and $\vec{G}=$ reciprocal lattice vector for a $2-\mathrm{D}$ photonic crystal that is given by: 


$$
\overrightarrow{\mathbf{G}}=\overrightarrow{\mathbf{b}}_{1}+\mathbf{m} \overrightarrow{\mathbf{b}}_{2}
$$

where $\vec{b}_{1}$ and $\vec{b}_{2}$ are the primitive reciprocal lattice vectors defined by the relation

$$
\vec{a}_{\mathbf{i}} \cdot \vec{b}_{\mathbf{j}}=\mathbf{2} \pi \delta_{\mathbf{j}}
$$

Where $\delta_{\mathrm{ij}}=$ Kronecker delta

$$
\delta_{i j}= \begin{cases}1, & i=j \\ 0, & i \neq j\end{cases}
$$

In the two-dimensional structure, two canonical highly symmetric lattice configurations exist: square and triangular. The primitive lattice vectors of a square lattice can be written as:

$$
\begin{array}{r}
\overrightarrow{\mathbf{a}}_{1}=\mathbf{a} \mathbf{x} \\
\overrightarrow{\mathbf{a}}_{2}=\mathbf{a} \hat{y}
\end{array}
$$

The unit cell of this periodic structure is the region enclosed by the square as shown in Figure 3.1 (a). Using the equation (3-4), the reciprocal primitive lattice vectors were determined as: 


$$
\vec{b}_{1}=\frac{2 \pi}{a} \hat{x}
$$

$$
\vec{b}_{2}=\frac{2 \pi}{a} \hat{y}
$$

In reciprocal space, the reciprocal primitive lattice vectors $\left(\vec{b}_{1}\right.$ and $\left.\vec{b}_{2}\right)$ and Brillouin zone are constructed as outline by a solid line shown in Figure 3.1 (b). Because of the symmetry of a square lattice, $1 / 8$ of the Brillouin zone is considered when calculating the band structure. This region is represented by a black shaded triangular region ГXM as shown in Figure 3.1 (b). All other points in the full Brillouin zone are mapped onto points within the irreducible zone by symmetry operation that include a $90^{\circ}$ rotation and mirror reflection. The $\mathrm{k}$ space points of high symmetry $\vec{\Gamma}, \overrightarrow{\mathrm{X}}$, and $\overrightarrow{\mathrm{M}}$ define the irreducible Brillouin zone and are given as:

$$
\begin{gathered}
\overrightarrow{\mathbf{\Gamma}}=\mathbf{0} \\
\overrightarrow{\mathbf{X}}=\frac{\pi}{\mathbf{a}}[\hat{\mathbf{x}}] \\
\overrightarrow{\mathbf{M}}=\frac{\pi}{\mathbf{a}}[\hat{\mathbf{x}}+\hat{\mathbf{y}}]
\end{gathered}
$$


Figure 3.2 (a), (b) represents the primitive lattice vector and reciprocal space of a 2-D photonic crystal with a triangular lattice of columns of high dielectric constant, ' $\varepsilon_{\mathrm{Al}}$ ' (black dots) surrounded in a background of low dielectric constant, ' $\varepsilon_{\text {air }}$ ' (white region).

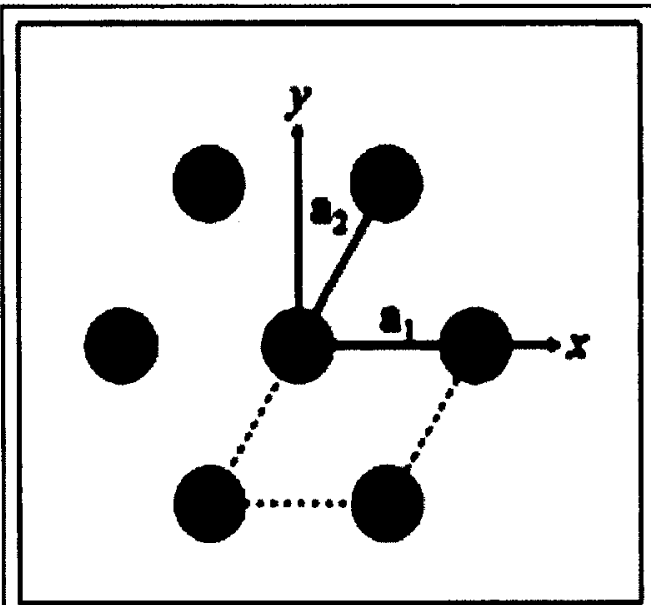

(a)

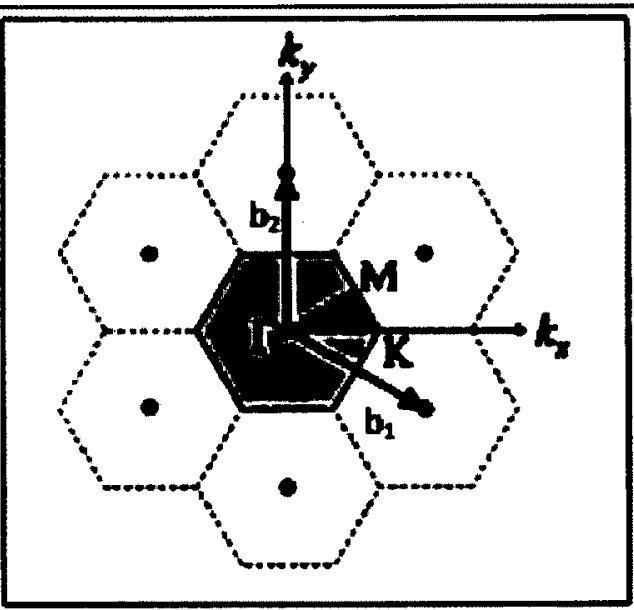

(b)

Figure 3.2: A 2-D photonic crystal with a triangular lattice of columns of high dielectric constant, ' $\varepsilon_{\mathrm{Al}}$ ' (black dots) surrounded in a background of low dielectric constant, ' $\varepsilon_{\text {air' }}$ ' (white region) (a) real space arrangement with two primitive lattice vectors $\vec{a}_{1}$ and $\vec{a}_{2} ;$ (b) reciprocal space with two reciprocal lattice vector $\left(\vec{b}_{1}\right.$ and $\left.\vec{b}_{2}\right)$; the Brillouin zone outlined by a solid bold border and the shaded triangular region with points ГKM represent the irreducible $1 / 12$ Brillouin zone.

The primitive lattice vectors of a triangular lattice can be written as:

$$
\begin{gathered}
\overrightarrow{\mathbf{a}}_{1}=\mathbf{a} \hat{\mathbf{x}} \\
\overrightarrow{\mathbf{a}}_{2}=\frac{\mathbf{a}}{2}[\hat{\mathbf{x}}+\sqrt{3} \hat{y}]
\end{gathered}
$$


The unit cell of this periodic structure is the region enclosed by the triangular as shown in Figure 3.2 (a). Using the equation (3-4), the reciprocal primitive lattice vectors are:

$$
\begin{gathered}
\vec{b}_{1}=\frac{2 \pi}{a} \hat{x}-\frac{\hat{y}}{\sqrt{3}} \\
\vec{b}_{2}=\frac{4 \pi}{\sqrt{3} a} \hat{y}
\end{gathered}
$$

In reciprocal space, the reciprocal primitive lattice vectors $\left(\vec{b}_{1}\right.$ and $\left.\vec{b}_{2}\right)$ and Brillouin zone are constructed as outlined by a solid line shown in Figure 3.2 (b). Because of the symmetry of the triangular lattice, $1 / 12$ of the Brillouin zone is considered when calculating the band structure. This region is represented by a black shaded triangular region $Г K M$ as shown in Figure 3.2 (b). The $\mathrm{k}$ space points of high symmetry $\vec{\Gamma}, \overrightarrow{\mathrm{M}}$, and $\overrightarrow{\mathrm{K}}$ define the irreducible Brillouin zone and are given as:

$$
\begin{gathered}
\overrightarrow{\boldsymbol{r}}=\mathbf{0} \\
\overrightarrow{\mathbf{M}}=\frac{\pi}{\mathrm{a}}\left[\hat{\mathbf{x}}+\frac{\hat{y}}{\sqrt{3}}\right]
\end{gathered}
$$




$$
\overrightarrow{\mathbf{K}}=\frac{4 \pi}{\sqrt{3} a} \hat{\mathbf{x}}
$$

To proceed with the plane wave method analysis for a photonic crystal, recalling the master equation (2-14), we write,

$$
\vec{H}(\vec{r})=\sum_{n} \hat{e}(n) \vec{H}(n) e^{i \vec{k} \cdot \vec{r}}
$$

Where $\hat{e}(n) ; n=1,2$ are unit polarization vectors for a given plane wave vector $\mathrm{k}$ in the Brillouin zone. Therefore, for transverse fields, we have,

$$
\mathrm{k} \cdot \hat{\mathrm{e}}(\mathrm{n})=0
$$

Let,

$$
f(\vec{r})=\frac{1}{\varepsilon(\vec{r})}
$$

Since, $\varepsilon(\vec{r})$ is a periodic function of the position vector $\vec{r}$, $f(\vec{r})$ will be periodic with $r$ as well. In terms of reciprocal lattice vector $\vec{G}$ as:

$$
f(\vec{r})=\sum_{G} f(\vec{G}) e^{i \vec{G} \cdot \vec{r}}
$$

Substitution of the equation (3-20) and (3-23) into equation (2-34), we have: 


$$
\begin{aligned}
& \sum_{n, \vec{G}}(\vec{k}+\vec{G}) \times f(\vec{G})[\vec{k} \times \hat{e}(n)] \vec{H}(n) e^{i(\vec{k}+\vec{G}) \cdot \vec{r}} \\
& =-\left(\frac{\omega}{c}\right)^{2} \sum_{n} \hat{e}(n) \vec{H}(n) e^{i \vec{k} \cdot \vec{r}}
\end{aligned}
$$

Multiplying both sides of equation (3-24) by $\hat{\mathrm{e}}(\hat{\mathrm{n}}) \mathrm{e}^{-\mathrm{i} \overrightarrow{\mathrm{k}}^{\prime} \cdot \overrightarrow{\mathrm{r}}}$ and then integrating over all space to get:

$$
\begin{aligned}
& \sum_{n, \vec{G}}(\vec{k}+\vec{G}) \times f(\vec{G})[k \times \hat{e}(n)] \cdot \hat{e}(\hat{n}) \vec{H}(n) \delta_{\vec{k}^{\prime}, \vec{k}+\vec{G}} \\
&=-\left(\frac{\omega}{c}\right)^{2} \sum_{n} \hat{e}(n) \cdot \hat{e}(\hat{n}) \vec{H}(n) \delta_{k, k} \\
& \sum_{n, \vec{G}}\left(\vec{k}^{\prime}\right) \times f(\vec{G})\left[\left(\vec{k}^{\prime}-\vec{G}\right) \times \hat{e}\left(\vec{k}^{\prime}-\vec{G}, n\right)\right] \cdot \hat{e}(\hat{n}) \vec{H}\left(\vec{k}^{\prime}-\vec{G}, n\right) \\
&=-\left(\frac{\omega}{c}\right)^{2} \sum_{n} \hat{e}(n) \cdot \hat{e}(\hat{n}) \vec{H}(n)
\end{aligned}
$$

Noting that $\hat{e}(n) \cdot \hat{e}(\hat{n})=\delta_{n, n}$, we obtain,

$$
\begin{aligned}
& \sum_{n, \vec{G}}\left(\vec{k}^{\prime}\right) \times f(\vec{G})\left[\left(\vec{k}^{\prime}-\vec{G}\right) \times \hat{e}\left(\vec{k}^{\prime}-\vec{G}, n\right)\right] \cdot \hat{e}(\hat{n}) \vec{H}\left(\vec{k}^{\prime}-\vec{G}, n\right) \\
& =-\left(\frac{\omega}{c}\right)^{2} \sum_{n} \delta_{n, h}, \vec{H}\left(\vec{k}^{\prime}, \hat{n}\right)
\end{aligned}
$$

Simplifying the right-hand side of the equation (3-27), 


$$
\begin{aligned}
& \sum_{n, \vec{G}}\left(\vec{k}^{\prime}\right) \times f(\vec{G})\left[\left(\vec{k}^{\prime}-\vec{G}\right) \times \hat{e}\left(\vec{k}^{\prime}-\vec{G}, n\right)\right] \cdot \hat{e}(\hat{n}) \vec{H}\left(\vec{k}^{\prime}-\vec{G}, n\right) \\
& =-\left(\frac{\omega}{c}\right)^{2} \vec{H}\left(\vec{k}^{\prime}, \hat{n}\right)
\end{aligned}
$$

Let $\overrightarrow{\mathrm{k}}^{\prime} \rightarrow \overrightarrow{\mathrm{k}}+\overrightarrow{\mathrm{G}}^{\prime}$,

$$
\begin{aligned}
& \sum_{n, \vec{G}}\left(\vec{k}+\vec{G}^{\prime}\right) \times f(\vec{G})\left[\left(\vec{k}+\vec{G}^{\prime}-\vec{G}\right) \times \hat{e}\left(\vec{k}+\vec{G}^{\prime}-\vec{G}, \hat{n}\right)\right] \\
& \cdot \hat{e}(\hat{n}) \overrightarrow{\vec{H}}\left(\vec{k}+\vec{G}^{\prime}-\vec{G}, n\right) \\
& =-\left(\frac{\omega}{c}\right)^{2} \vec{H}\left(\vec{k}+\vec{G}^{\prime}, \hat{n}\right)
\end{aligned}
$$

Let, $\overrightarrow{\mathrm{G}}^{\prime}-\overrightarrow{\mathrm{G}} \rightarrow \overrightarrow{\mathrm{G}}^{\prime}$

$$
\begin{aligned}
& \sum_{n, \vec{G}^{\prime}}\left[\hat{e}\left(\vec{k}+\vec{G}^{\prime \prime}, \hat{n}^{\prime}\right) \cdot\left(\vec{k}+\vec{G}^{\prime}\right)\right] \times f\left(\vec{G}^{\prime}-\vec{G}^{\prime \prime}\right)\left[\left(\vec{k}+\vec{G}^{\prime \prime}\right) \times \hat{e}\left(\vec{k}+\vec{G}^{\prime \prime}, \dot{n}\right)\right] \vec{H}\left(\vec{k}+\vec{G}^{\prime \prime}, \hat{n}^{\prime}\right) \\
& =-\left(\frac{\omega}{c}\right)^{2} \vec{H}\left(\vec{k}+\vec{G}^{\prime \prime}, \hat{n}\right)
\end{aligned}
$$

To match the results presented in the literature ${ }^{\prime}$, let $\overrightarrow{\mathrm{G}}^{\prime} \rightarrow \overrightarrow{\mathrm{G}}$ and $\overrightarrow{\mathrm{G}}^{\prime \prime} \rightarrow \overrightarrow{\mathrm{G}}^{\prime}$ and renaming $\mathrm{n} \rightarrow \mathrm{n}$ and $\mathrm{n} \rightarrow$ ń, we have, 


$$
\begin{aligned}
& \sum_{n}\left[\vec{G}^{\prime}\right. \\
& \left.\left.=-\left(\frac{\hat{e}}{c}\right)^{2} \vec{k}+\vec{G}^{\prime}, n\right) \cdot(\vec{k}+\vec{G})\right] \times f\left(\vec{G}-\vec{G}^{\prime}\right)\left[\left(\vec{k}+\vec{G}^{\prime}\right) \times \hat{e}\left(\vec{k}+\vec{G}^{\prime}, \hat{n}\right)\right] \overrightarrow{\vec{H}}\left(\vec{k}+\vec{G}^{\prime}, \hat{n}\right) \\
&
\end{aligned}
$$

Using vector identity as:

$$
\vec{A} \cdot(\vec{B} \times \vec{C} \times \vec{D})=(\vec{A} \times \vec{B}) \cdot(\vec{C} \times \vec{D})
$$

In equation (3.31) gives:

$$
\begin{aligned}
& \sum_{n^{\prime} \vec{G}^{\prime}}[(\vec{k}+\vec{G}) \times \hat{e}(\vec{k}+\vec{G}, n)] \cdot\left[\left(\vec{k}+\vec{G}^{\prime}\right) \times \hat{e}\left(\vec{k}+\vec{G}^{\prime}, \hat{n}\right)\right] f\left(k+\vec{G}^{\prime}\right) \vec{H}\left(\vec{k}+\vec{G}^{\prime}, \hat{n}\right) \\
& =\left(\frac{\omega}{c}\right)^{2} \vec{H}(\vec{k}+\vec{G}, n)
\end{aligned}
$$

We have obtained a linear equation ${ }^{1}$ as:

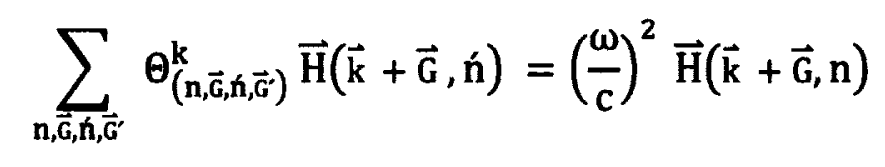

where,

$$
\theta_{(n, G, n, G)}^{k}=[(\vec{k}+\vec{G}) \times \hat{e}(\vec{k}+\vec{G}, n)] \cdot\left[\left(\vec{k}+\vec{G}^{\prime}\right) \times \hat{e}\left(\vec{k}+\vec{G}^{\prime}, \hat{n}\right)\right] f\left(\vec{G}-\vec{G}^{\prime}\right)
$$

Equation (3-35) is used to find the eigenvalues for a three-dimensional photonic crystal. For a given $\overrightarrow{\mathrm{k}}$ and $\mathrm{N}$ reciprocal lattice vector $\vec{G}_{1,2,3, \ldots \ldots . N}$, three-dimensional, linear, Hermitian matrix $\Theta$ will be of dimension $2 N \times 2 N$ with following matrix structure: 


$$
\left[\begin{array}{cccccc}
\Theta_{\left(n_{1}, G_{1}, n_{1}, G_{1}\right)}^{\mathrm{k}} & \Theta_{\left(n_{1}, G_{1}, n_{2}, G_{1}\right)}^{\mathrm{k}} & \Theta_{\left(n_{1}, G_{1}, n_{1}, G_{2}\right)}^{\mathrm{k}} & \Theta_{\left(n_{1}, G_{1}, n_{2}, G_{2}\right)}^{\mathrm{k}} & \cdots & \Theta_{\left(n_{1}, G_{1}, n_{2}, G_{N}\right)}^{\mathrm{k}} \\
\Theta_{\left(n_{2}, G_{1}, n_{1}, G_{1}\right)}^{\mathrm{k}} & \Theta_{\left(n_{2}, G_{1}, n_{2}, G_{1}\right)}^{\mathrm{k}} & \Theta_{\left(n_{2}, G_{1}, n_{1}, G_{2}\right)}^{\mathrm{k}} & \Theta_{\left(n_{2}, G_{1}, n_{2}, G_{2}\right)}^{\mathrm{k}} & \cdots & \Theta_{\left(n_{2}, G_{1}, n_{2}, G_{N}\right)}^{\mathrm{k}} \\
\Theta_{\left(n_{1}, G_{2}, n_{1}, G_{1}\right)}^{\mathrm{k}} & \left.\Theta_{\left(n_{1}, G_{2}, n_{2}, G_{1}\right)}^{\mathrm{k}}\right) & \Theta_{\left(n_{1}, G_{2}, n_{1}, G_{2}\right)}^{\mathrm{k}} & \Theta_{\left(n_{1}, G_{2}, n_{2}, G_{2}\right)}^{\mathrm{k}} & \cdots & \Theta_{\left(n_{1}, G_{2}, n_{2}, G_{N}\right)}^{\mathrm{k}} \\
\Theta_{\left(n_{2}, G_{2}, n_{1}, G_{1}\right)}^{\mathrm{k}} & \Theta_{\left(n_{2}, G_{2}, n_{2}, G_{1}\right)}^{\mathrm{k}} & \Theta_{\left(n_{2}, G_{2}, n_{1}, G_{2}\right)}^{\mathrm{k}} & \Theta_{\left(n_{2}, G_{2}, n_{2}, G_{2}\right)}^{\mathrm{k}} & \cdots & \Theta_{\left(n_{2}, G_{2}, n_{2}, G_{N}\right)}^{\mathrm{k}} \\
\vdots & \vdots & \vdots & \vdots & \ddots & \vdots \\
\Theta_{\left(n_{2}, G_{N}, n_{1}, G_{1}\right)}^{\mathrm{k}} & \Theta_{\left(n_{2}, G_{N}, n_{2}, G_{1}\right)}^{\mathrm{k}} & \Theta_{\left(n_{2}, G_{N}, n_{1}, G_{2}\right)}^{\mathrm{k}} & \Theta_{\left(n_{2}, G_{N}, n_{2}, G_{2}\right)}^{\mathrm{k}} & \cdots & \Theta_{\left(n_{2}, G_{N}, n_{2}, G_{N}\right)}^{\mathrm{k}}
\end{array}\right]
$$

In two-dimensional analysis, the electromagnetic waves are resolved with two polarizations, namely transverse magnetic (TM) and transverse electric (TE). These polarizations are relative to the plane of periodicity of the photonic crystal and make several simplifications to the k-dependent Hermitian matrix $\theta$ given in equation (3-35). Only TM polarization is considered for this thesis work. In 2-D, a simplified form ${ }^{19}$ of the Hermitian matrix $\Theta$ to compute eigenvalues for TM polarization is:

$$
\boldsymbol{\theta}_{(\mathrm{TM})}^{\mathbf{k}}=|\overrightarrow{\mathbf{k}}+\overrightarrow{\mathbf{G}}|\left|\overrightarrow{\mathbf{k}}+\overrightarrow{\mathbf{G}}^{\prime}\right| \mathbf{f}\left(\overrightarrow{\mathbf{G}}-\overrightarrow{\mathbf{G}}^{\prime}\right)
$$

A 2-D photonic crystal structure was simulated with the help of equation (3-37). Departmental researchers ${ }^{26}$ develop these tools to compute photonic band structures and photonic bandgap maps.

General steps carried out by a simulator tool for plane wave analysis are:

i. Define the real space lattice vector and lattice constant for a 2-D photonic crystal.

ii. Define various dielectric lattice parameters, such as the dielectric constant of the dielectric column ' $\varepsilon_{A l}$ ' provided by the manufacturer and the dielectric background ' $\varepsilon_{\text {air }}$ ', rod radius, ' $r$ ' which is constant. 
iii. Specify the filling factor ' $f_{f}$ ' for the lattice under review and the area of the primitive unit cell calculated.

iv. Generate primitive reciprocal space lattice vector for the photonic crystal.

v. The reciprocal vector, ' $\vec{G}$ ' used belongs to a discrete approximation in a circular region in k-space bounded by some maximum value (largest reciprocal vector allowed for desired number of plane waves)

vi. The reciprocal space trajectory and number of steps along each $\mathrm{k}$-space trajectory are defined.

vii. These trajectories trace out the perimeter of the Brillouin zone between points of high symmetry.

viii. The Hermitian matrix $\Theta_{(T M)}^{\mathrm{k}}$ considered for each $\mathrm{k}$-space point is solved to obtain the eigen frequencies. The collection of these eigen frequencies (TM mode) as a function of $\mathrm{k}$-space defines the photonic band structure.

\subsubsection{Photonic Band Structure for a 2-D Photonic Crystal: TM Polarized Mode}

Primitive lattice vectors, reciprocal space, and Brillouin zone are already discussed for a triangular lattice in the previous section. The irreducible portion of the Brillouin zone defines the k-space trajectory to be followed while calculating the photonic band structure. The periodic real reciprocal dielectric function $f(r)$ can be expanded in a Fourier series in terms of lattice reciprocal vectors as: 


$$
\mathbf{f}(\overrightarrow{\mathbf{r}})=\sum_{\overrightarrow{\mathbf{G}}} \mathbf{f}(\overrightarrow{\mathbf{G}}) \mathbf{e}^{\mathbf{i} \overrightarrow{\mathbf{G}} \cdot \overrightarrow{\mathbf{r}}}
$$

$f(\vec{G})=$ Fourier expansion coefficient. The compact form of the Fourier expansion coefficient in k-space can be written as:

$$
f_{\text {triangular }}(\vec{G})= \begin{cases}\frac{1}{\varepsilon_{\text {air }}}+\left(\frac{1}{\varepsilon_{\mathrm{Al}}}-\frac{1}{\varepsilon_{\text {alr }}}\right) f_{\mathrm{f}}, & \overrightarrow{\mathbf{G}}=0 \\ \left(\frac{1}{\varepsilon_{\mathrm{Al}}}-\frac{1}{\varepsilon_{\mathrm{alr}}}\right) \mathrm{I}(\overrightarrow{\mathrm{G}}), & \overrightarrow{\mathbf{G}} \neq \mathbf{0}\end{cases}
$$

where, $f_{\mathrm{f}}$ is the filling factor for the triangular lattice of dielectric columns and is given by:

$$
\mathbf{f}_{\mathbf{f}}=\frac{\mathbf{A}_{\mathbf{c}}}{\mathbf{A}_{\text {unit }}}
$$

$\mathbf{A}_{\mathbf{c}}$ is the cross sectional area of the dielectric column;

$\mathbf{A}_{\text {unit }}$ is the area of the primitive unit cell of the lattice

I (G) is a geometry factor and is defined as:

$$
I(G)=2 f_{f} \frac{l_{1}(G R)}{G R} ;
$$

$\mathbf{J}_{\mathbf{1}}$ is the first order Bessel function of the first kind. 
For a 2-D photonic crystal with a triangular lattice, a simulation run was conducted to obtain a photonic band structure as shown in Figure 3.3. The dielectric constant of the dielectric columns were considered as $\varepsilon_{\mathrm{Al}}=9.2$, while the background dielectric as air, with a dielectric constant, $\varepsilon_{\text {air }}=1$. The filling factor was taken to be $f_{f}=$ 0.0642. This value of filling factor corresponded to columns of radius, $r=0.143 \mathrm{a}$. The reciprocal vectors used in the calculation are chosen from all those reciprocal vectors contained within a circular region in k-space. A total of 961 plane waves were used to obtain the resulting photonic band structure. This photonic band structure shows three ranges of forbidden normalized frequencies $(0.36-0.55),(0.65-0.72)$ and $(0.92-0.96)$ as depicted in Figure 3.3.

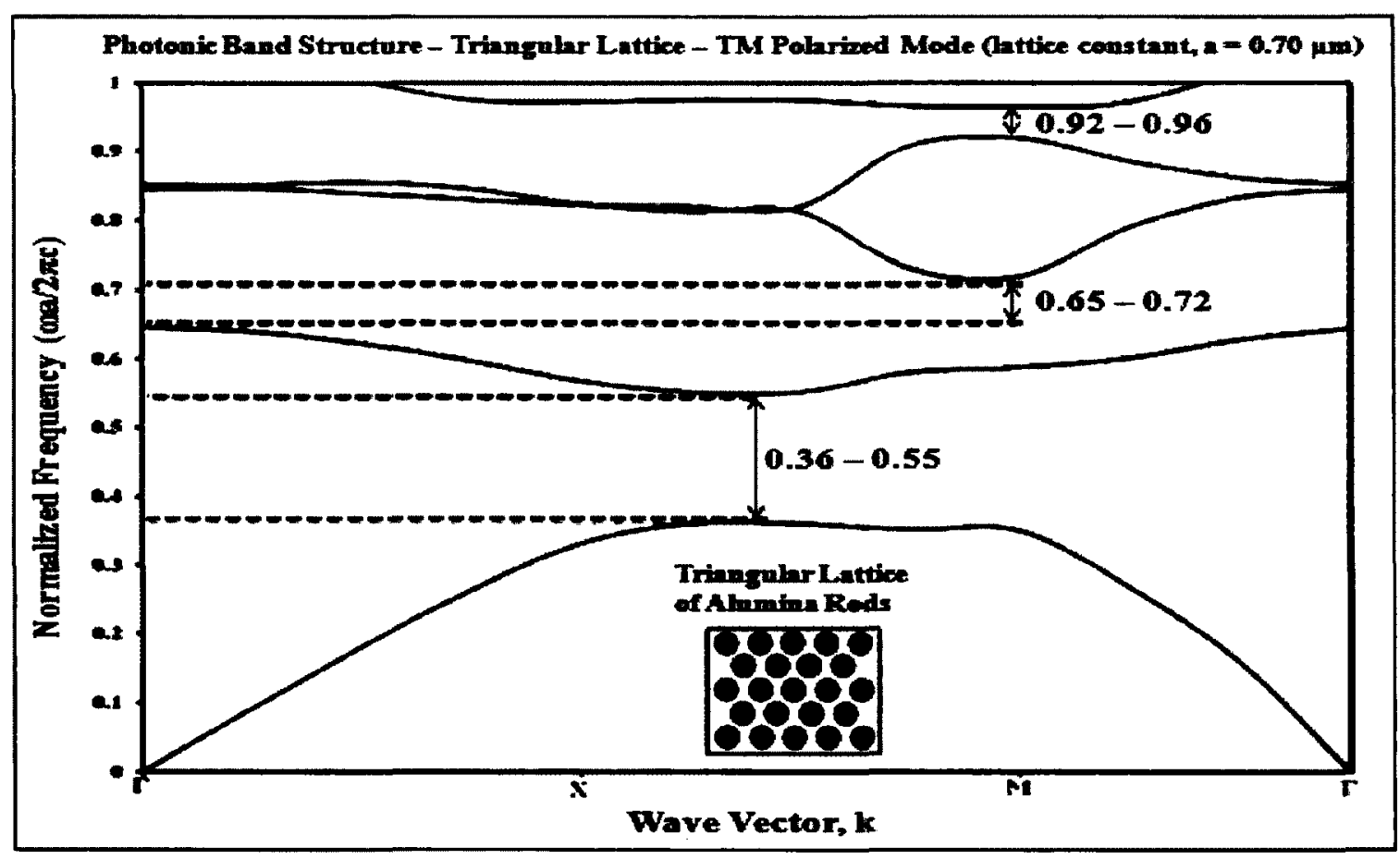

Figure 3.3: The photonic band structure of the two-dimensional triangular lattice of dielectric columns (TM polarized mode). The photonic bandgap indicated in the figure shows the range of forbidden frequencies. Inset shows arrangement of alumina rods in a triangular lattice. 


\subsubsection{Photonic Bandgap Maps:}

While designing photonic crystal devices, it is necessary to determine the photonic crystal parameters (photonic bandgap) from known frequency characteristics. In order to solve this problem, photonic bandgap maps are created with the help of a plane wave simulation tool. The photonic bandgap map is a visualization of the lower- and upperband edges for the gaps of different orders and polarization. The band above the photonic bandgap is commonly referred to as the air band, and the band below a photonic bandgap is referred to as the dielectric band ${ }^{1}$. It displays a region of a complete photonic bandgap for a range of frequency that is the intersection of the TE and TM gaps. After constructing photonic bandgap maps, what minimum and maximum values of lattice constants with a higher and lower edge of photonic bandgaps should be considered? What is the optimal lattice constant that maximizes the gap size as a function of the index contrast? To answer these questions, photonic bandgap maps for a 2-D photonic crystal with a triangular lattice for both TM and TE polarizations are examined.

Consider a 2-D photonic crystal consisting of parallel columns (alumina rod) arranged in a triangular lattice in transverse magnetic (TM) polarized mode. The dielectric columns (alumina rods) have a constant radius, ' $r=0.1 \mu \mathrm{m}$ '. A plane wave simulator tool using 961 plane waves generates all photonic bandgap maps by varying lattice constant, ' $a$ '. The gap maps show gaps as a function of the lattice constant for a fixed dielectric ratio of 9.2:1. Appendix A provides brief information about the location and size of the photonic bandgap map in the transverse electric (TE) polarized mode for a 
2-D photonic crystal with a triangular lattice, and also some plots showing the location of the complete bandgaps outlined for both TM and TE polarizations.

Figure 3.4 shows a plot (photonic bandgap map) between the normalized frequency, $\left(\frac{\omega \mathrm{a}}{2 \pi \mathrm{c}}\right)$, versus different values of the lattice constant, ' $\mathrm{a}$ ' $(0.22 \mu \mathrm{m}-0.88 \mu \mathrm{m})$ for a triangular lattice of alumina rods, $\varepsilon_{\mathrm{Al}}=9.2 \mathrm{in} \mathrm{TM}$ polarization. In the microwave regime, angular frequency, ' $\omega$ ', and lattice constant, ' $a$ ', must be scaled to the desired levels, using the scalability law explained in Chapter 2, Section 2.3. As the value of the lattice constant increases, the bandgap size increases that are almost closing at lower values of ' $a$ ' and continues until the ' $a=0.88 \mu \mathrm{m}$ '.

From this plot, a particular value of the lattice constant, ' $a=0.70 \mu \mathrm{m}$ ' is considered that has two band gaps at normalized frequencies; $0.36-0.55$ (corresponding to the range of wavelength, $\lambda=1.27 \mu \mathrm{m}$ to $1.98 \mu \mathrm{m}$ ) with a gap/mid-gap ratio of $40 \%$ and second gap at $0.65-0.72$ (corresponding to the range of wavelength, $\lambda=0.97 \mu \mathrm{m}$ to $1.09 \mu \mathrm{m}$ ) with a gap/mid-gap ratio of $11 \%$ as shown by arrowhead lines (black). This chosen value of the lattice constant is further used to design and fabricate a 2-D photonic crystal linear waveguide with triangular lattice both in the optical and microwave regimes. 


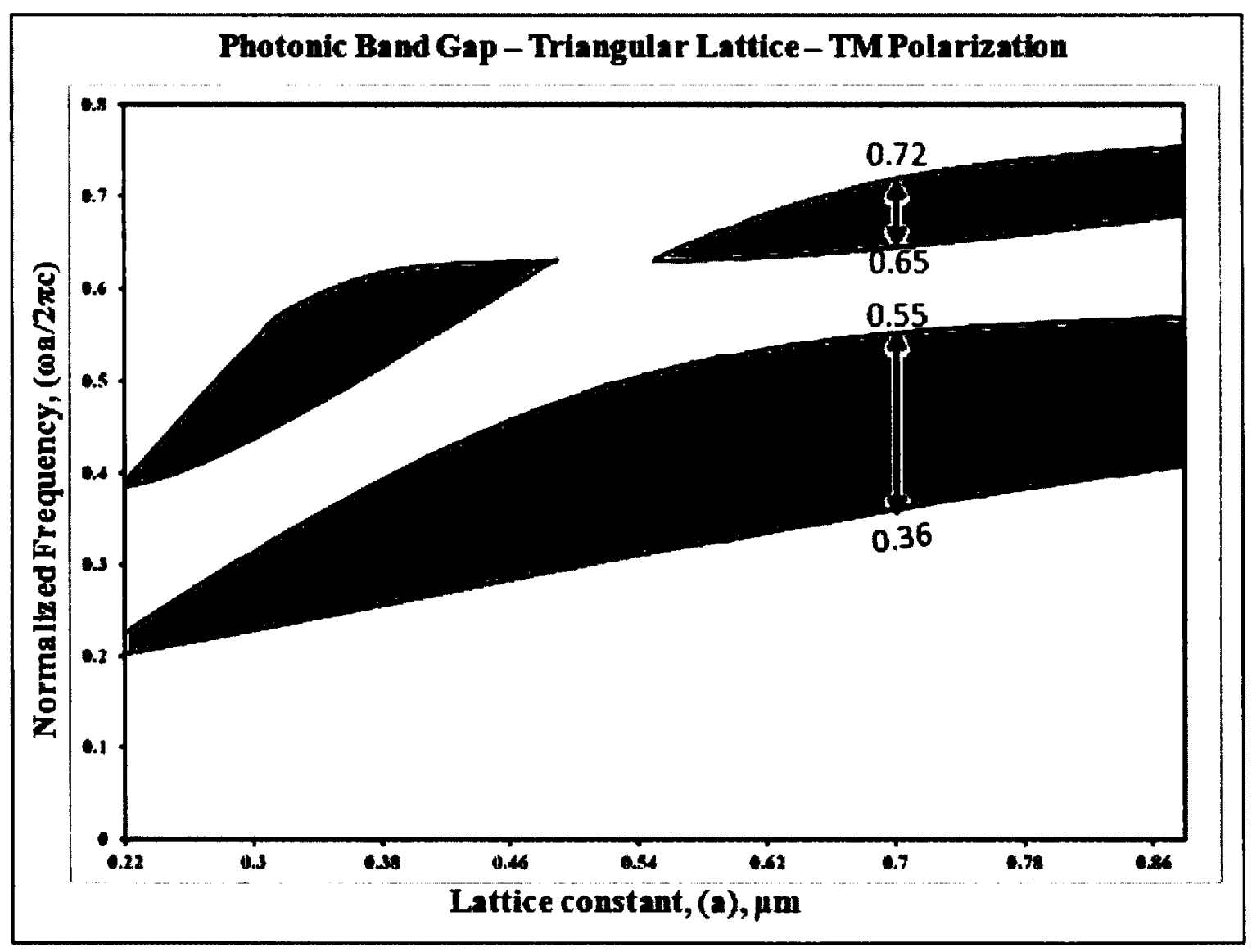

Figure 3.4: Plot of normalized frequency, $\left(\frac{\omega a}{2 \pi c}\right)$ versus lattice constant ' $a$ '. Photonic bandgaps (black) are shown for a two-dimensional photonic crystal (TM polarization) with triangular lattice and dielectric rods $\left(\varepsilon_{\mathrm{N}}=9.2\right)$ with a background as air $\left(\varepsilon_{\text {air }}=1\right)$. Rod radius is constant, ' $r=0.1 \mu \mathrm{m}$ ' and the chosen value of the lattice constant, ' $a=0.70 \mu \mathrm{m}$ '.

From this photonic bandgap map, a chosen value of lattice constant, ' $a=0.70 \mu \mathrm{m}$ ' was considered to perform optical analysis with the supercell technique and FDTD method, and later, using scalability law, constructing a 2-D triangular lattice template in the microwave regime. This value of lattice constant showed a complete bandgap for both TE and TM polarizations as discussed in Appendix A. 
In the next sub-section, a 2-D photonic crystal with triangular lattice is evaluated by a supercell technique based on plane wave analysis. With this technique, a single mode waveguide is designed.

\subsubsection{Supercell Technique:}

The standard plane wave expansion method is limited to the calculation of band structure for large photonic crystals, such as cavities and waveguides. In the supercell technique, the unit cell is replaced by an extended unit cell while preserving the periodicity. In this method, a 2-D photonic crystal structure with a triangular lattice (TM polarization) is considered as shown in Figure 3.5 with a linear waveguide (W1) by removing one row of rods in $\mathrm{X}$ direction, regarded as the supercell. The construction of this supercell then repeats along the different spatial directions. Thus, the artificial structure is defined to consist of periodic defects separated by a region of perfect photonic crystal. The eigen frequencies and the eigen functions of the defect mode are calculated. 


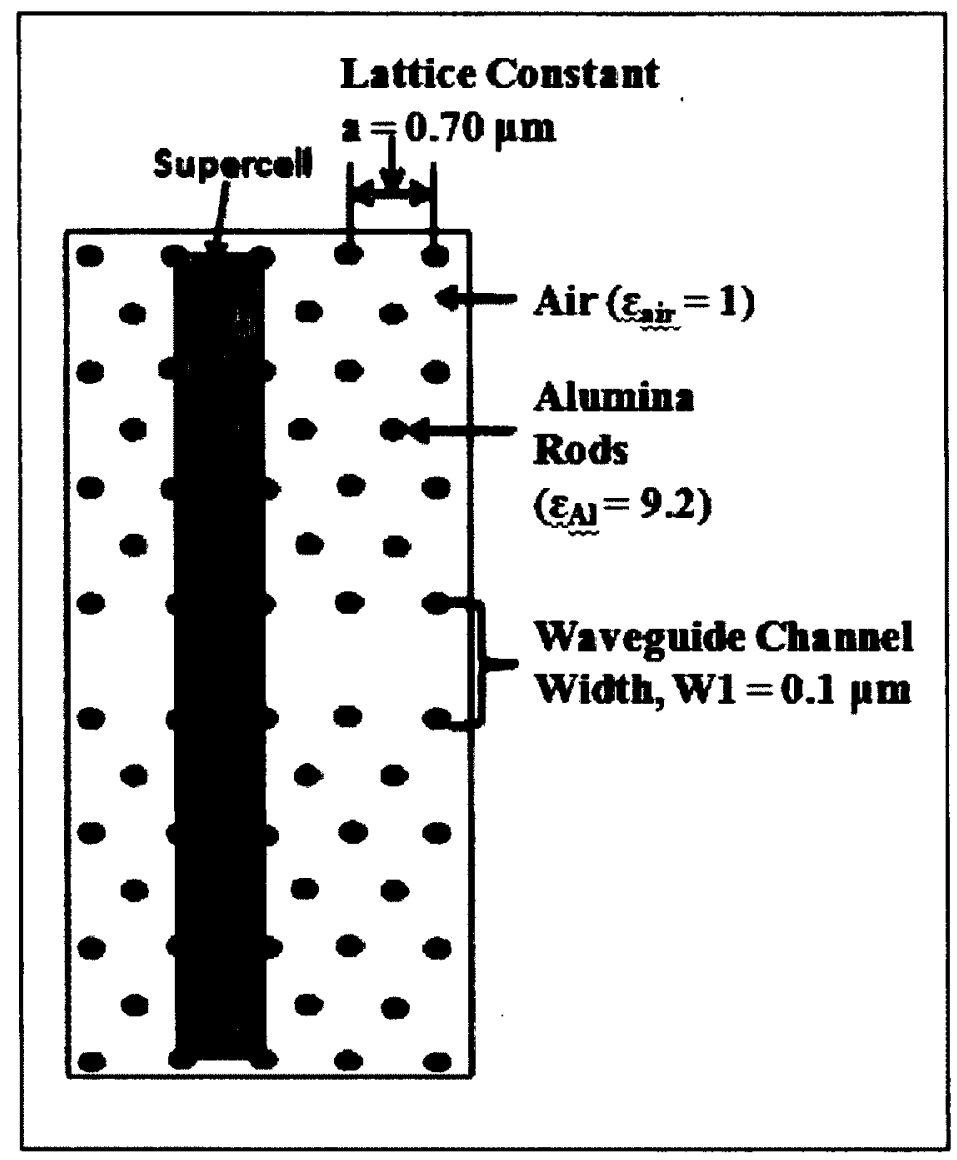

Figure 3.5: An array of 2-D triangular lattice photonic crystal with a line defect in the $\mathrm{X}$ direction. The shaded region represents the supercell.

Figure 3.6 shows the $\Gamma-X$ portion of the dispersion curve computed using 6,561 plane waves. A single band line at normalized frequencies of $0.58-0.74$ is observed in the original bandgap and forms the mode of the linear waveguide. 


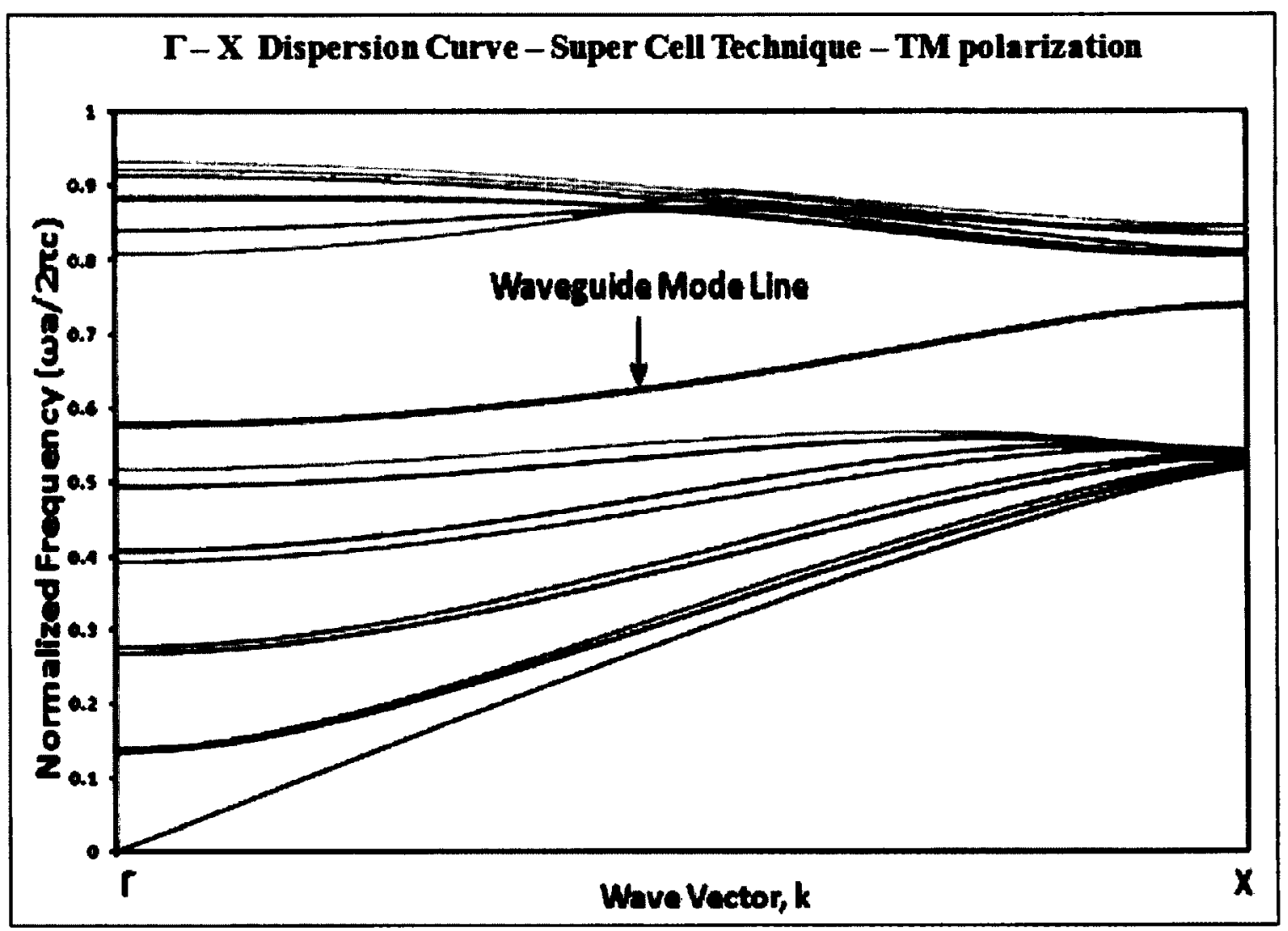

Figure 3.6: $\Gamma-\mathrm{X}$ dispersion curve of 2-D line defect waveguide photonic crystal with triangular lattice having lattice constant, $\mathrm{a}=0.70 \mu \mathrm{m}, \mathrm{TM}$ polarization obtained with the supercell technique. Single guided modes at normalized frequencies between $0.58-0.74$ within the photonic bandgap is present.

For this range of normalized frequencies, further investigation was carried out by implementing another simulation method known as the finite difference time domain (FDTD) method. All plane wave method results were verified by FDTD. In the next section, the FDTD method is presented and simulations of a 2-D photonic crystal with a triangular lattice (TM polarization) having the same parameters as specified in the plane wave method are obtained. 


\subsection{Finite Difference Time Domain (FDTD) Method:}

The FDTD method has been one of the most widely used computational techniques for solving electromagnetic boundary problems ${ }^{26,31,33,34,38}$. In 1966, K.Yee ${ }^{56}$ introduced the FDTD method that formulated Maxwell's equations in the time domain. In this method, Maxwell's equations were fully discretized in both space and time by approximating a centered two point finite difference. A few of the main advantages that make FDTD a powerful and versatile simulation tool are: flexibility and capability of analyzing complex scattering structures, easy implementation, visualizing the time varying fields with the volume of space, handling non-linear, frequency dependent, and obtaining broad spectral information by a single run. It requires a large memory space as it is linearly proportional to the volume of the simulated structure.

The FDTD simulation tool developed by our departmental researchers ${ }^{26}$ offer appropriate boundary conditions and sources. When the source is excited and set to be a continuous wave, it is propagated through the structure by time stepping through the entire grid repeatedly. Some boundary reflection took place in the computational domain. J.P. Berenger ${ }^{57}$ introduced the perfectly matched layer (PML) to eliminate/suppress these unwanted reflections. In PML, the field is exponentially decaying so it propagates to the grid edge. Throughout the coming sub-sections, the development of the FDTD method is followed closely as outlined in the book by Dennis M. Sullivan ${ }^{4}$. 


\subsubsection{Formulation of Finite Difference Time Domain (FDTD) Method:}

Maxwell's electromagnetic curl equations are the main root for the development of the FDTD algorithm. When there are no charges, no current and the medium is nonmagnetic, that is $\varepsilon_{\mathrm{r}}$ real and $\mu_{\mathrm{r}}=1$, then Maxwell's equations can be written as:

$$
\begin{aligned}
& \frac{\partial \overrightarrow{\mathrm{E}}}{\partial \mathrm{t}}=\frac{1}{\varepsilon_{0}}(\vec{\nabla} \times \overrightarrow{\mathrm{H}}) \\
& \frac{\partial \overrightarrow{\mathrm{H}}}{\partial \mathrm{t}}=-\frac{1}{\mu_{0}}(\vec{\nabla} \times \overrightarrow{\mathrm{E}})
\end{aligned}
$$

In general, the electric field is many orders of magnitude larger than the magnetic field. To adjust for the imbalance, the electric field is recalled ${ }^{4}$ :

$$
\begin{gathered}
\widetilde{E}=\sqrt{\frac{\varepsilon_{0}}{\mu_{0}}} \overrightarrow{\mathrm{E}} \\
\frac{\partial \widetilde{\mathrm{E}}}{\partial \mathrm{t}}=\frac{1}{\varepsilon_{\mathrm{r}} \sqrt{\varepsilon_{0} \mu_{0}}}(\vec{\nabla} \times \overrightarrow{\mathrm{H}}) \\
\frac{\partial \overrightarrow{\mathrm{H}}}{\partial \mathrm{t}}=-\frac{1}{\sqrt{\varepsilon_{0} \mu_{0}}} \vec{\nabla} \times \widetilde{\mathrm{E}}
\end{gathered}
$$


While formulating the FDTD algorithm, equations (3-45) and (3-46) are discretized in the time and space domain. These two equations provide the six field component; $\widetilde{\mathrm{E}}_{\mathbf{x}}, \widetilde{\mathrm{E}}_{\mathbf{y}}, \widetilde{\mathrm{E}}_{\mathrm{z}}, \mathrm{H}_{\mathbf{x}}, \mathrm{H}_{\mathbf{y}}, \mathrm{H}_{\mathbf{z}}$ as:

$$
\begin{aligned}
& \frac{\partial \widetilde{\mathrm{E}}_{\mathrm{x}}}{\partial \mathrm{t}}=\frac{1}{\varepsilon_{\mathrm{r}} \sqrt{\varepsilon_{0} \mu_{0}}}\left(\frac{\partial \mathrm{H}_{\mathrm{z}}}{\partial \mathrm{y}}-\frac{\partial \mathrm{H}_{\mathrm{y}}}{\partial \mathrm{z}}\right) \\
& \frac{\partial \widetilde{\mathrm{E}}_{\mathrm{y}}}{\partial \mathrm{t}}=\frac{1}{\varepsilon_{\mathrm{r}} \sqrt{\varepsilon_{0} \mu_{0}}}\left(\frac{\partial \mathrm{H}_{\mathrm{x}}}{\partial \mathrm{z}}-\frac{\partial \mathrm{H}_{\mathrm{z}}}{\partial \mathrm{x}}\right) \\
& \frac{\partial \widetilde{\mathrm{E}}_{\mathrm{z}}}{\partial \mathrm{t}}=\frac{1}{\varepsilon_{\mathrm{r}} \sqrt{\varepsilon_{0} \mu_{0}}}\left(\frac{\partial \mathrm{H}_{\mathrm{y}}}{\partial \mathrm{x}}-\frac{\partial \mathrm{H}_{\mathrm{x}}}{\partial \mathrm{y}}\right) \\
& \frac{\partial \mathrm{H}_{\mathrm{x}}}{\partial \mathrm{t}}=\frac{1}{\sqrt{\varepsilon_{0} \mu_{0}}}\left(\frac{\partial \tilde{\mathrm{E}}_{\mathrm{z}}}{\partial \mathrm{y}}-\frac{\partial \tilde{\mathrm{E}}_{\mathrm{y}}}{\partial \mathrm{z}}\right) \\
& \frac{\partial \mathrm{H}_{\mathrm{y}}}{\partial \mathrm{t}}=\frac{1}{\sqrt{\varepsilon_{0} \mu_{0}}}\left(\frac{\partial \tilde{\mathrm{E}}_{\mathrm{x}}}{\partial \mathrm{z}}-\frac{\partial \tilde{\mathrm{E}}_{\mathrm{z}}}{\partial \mathrm{x}}\right) \\
& \frac{\partial \mathrm{H}}{\sqrt{\varepsilon_{0} \mu_{0}}}\left(\frac{1}{\partial \mathrm{x}}-\frac{\partial \tilde{\mathrm{E}}_{\mathrm{x}}}{\partial \mathrm{y}}\right)
\end{aligned}
$$

In a three-dimensional structure, the six components are coupled. This thesis work is concerned with the 2-D photonic crystal, thus the formulation of the FDTD algorithm is restricted to the $(\mathrm{X}, \mathrm{Y})$ axis. The 2-D formulation is highly suitable for 
analyzing the planar optical properties of the photonic crystal. Equation $3.47(a-f)$ is decoupled into 2 sets; transverse magnetic (TM) with components $\left(H_{x}, H_{y}, \tilde{E}_{z}\right)$ and transverse electric (TE) with $\left(\tilde{E}_{x}, \tilde{E}_{y}, H_{z}\right)$. Again, only TM polarization is considered and requires only the discretization of $(3.47 \mathrm{c}),(3.47 \mathrm{~d})$ and $(3.47 \mathrm{e})$. Rewriting these equations with the $Z$ dependence removed ${ }^{4}$ as:

$$
\begin{gathered}
\frac{\partial \widetilde{\mathrm{E}}_{\mathrm{z}}}{\partial \mathrm{t}}=\frac{1}{\varepsilon_{\mathrm{r}} \sqrt{\varepsilon_{0} \mu_{0}}}\left(\frac{\partial \mathrm{H}_{\mathrm{y}}}{\partial \mathrm{x}}-\frac{\partial \mathrm{H}_{\mathrm{x}}}{\partial \mathrm{y}}\right) \\
\frac{\partial \mathrm{H}_{\mathrm{x}}}{\partial \mathrm{t}}=-\frac{1}{\sqrt{\varepsilon_{0} \mu_{0}}}\left(\frac{\partial \tilde{\mathrm{E}}_{\mathrm{z}}}{\partial \mathrm{y}}\right) \\
\frac{\partial \mathrm{H}_{\mathrm{y}}}{\partial \mathrm{t}}=\frac{1}{\sqrt{\varepsilon_{0} \mu_{0}}}\left(\frac{\partial \tilde{\mathrm{E}}_{\mathrm{z}}}{\partial \mathrm{x}}\right)
\end{gathered}
$$

The grid used to discretize the fields is known as the Yee cell introduced by $\mathrm{K} . \mathrm{Yee}^{56}$. This grid is represented in a spatial formation of field variables as shown in Figure 3.7. The grid is defined such that $\Delta x=\Delta y$ with the $\widetilde{\mathrm{E}}_{\mathrm{z}}$ field component placed on the (i, j) grid points; the $H_{x}$ component is displaced by 0.5 on the $j$ 's and the $H_{y}$ is displaced by 0.5 on the i's. 


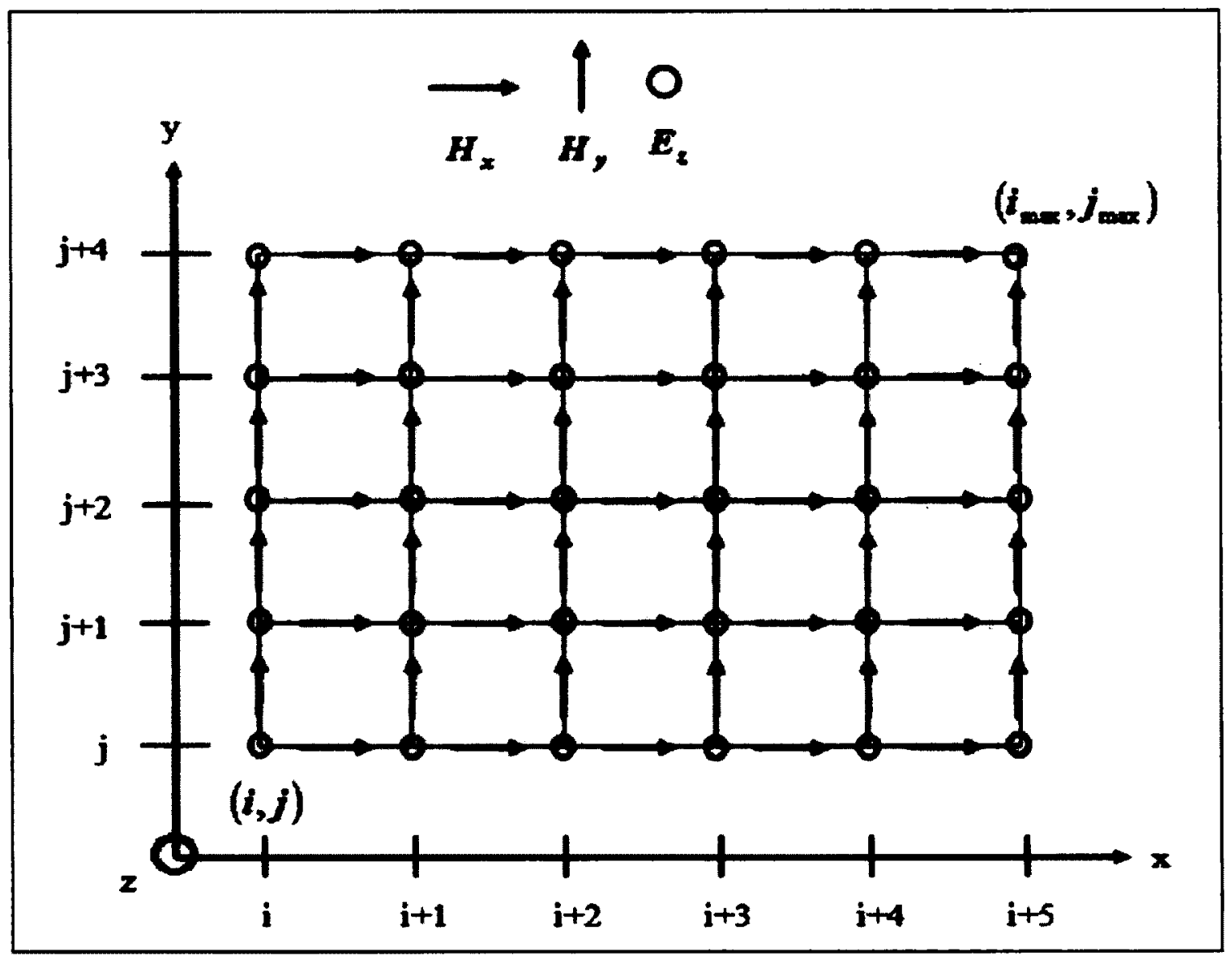

Figure 3.7: Spatial arrangement of field variables in the FDTD grid for two dimensions (TM polarized).

The $\mathrm{H}_{\mathbf{x}}$ magnetic field component points in the $\mathbf{x}$ direction and is specified at grid points $\mathrm{i}$ and at midway points between the $\mathrm{j}$ grid points. Thus,

$$
H_{x} \rightarrow H_{x}(i, j+0.5)
$$

The $\mathrm{H}_{\mathrm{y}}$ magnetic field component points in the $\mathrm{y}$ direction and is specified at grid points $\mathrm{j}$ and at midway points between the $\mathrm{i}$ grid points. Thus,

$$
\mathrm{H}_{\mathrm{y}} \rightarrow \mathrm{H}_{\mathbf{y}}(\mathrm{i}+0.5, \mathrm{j})
$$

In the spatial discretization process, the nearest neighbor values are used to determine derivatives. In the temporal discretization process, present and text time 
values are used to determine the derivatives. Also, temporal integrals can be evaluated by keeping track of a field summation over time. The $\tilde{E}_{z}$ and $\left(H_{x}, H_{y}\right)$ field components are incremented by $\Delta t$ time steps. The discretization grid and time step process allows the time and spatial derivatives of equations (3-48), (3-49) and (3-50) to be reconstructed into the finite difference formulation. The $\tilde{E}_{z}$ field component is represented as;

$$
\frac{\widetilde{E}_{z}(i, j, n+0.5)-(i, j, n-0.5)}{\Delta t}=\frac{1}{\sqrt{\varepsilon_{0} H_{0}}}\left(\frac{H_{y}(i+0.5, j, n)-H_{y}(i-0.5, j, n)}{\Delta x}\right)-\frac{1}{\sqrt{\varepsilon_{0} \mu_{0}}}\left(\frac{H_{x}(i, j+0.5, n)-H_{x}(i, j-0.5, n)}{\Delta x}\right)
$$

The next value of $\tilde{E}_{z}$ can be isolated (3-51) and expressed in terms of known field components that are 0.5 time intervals earlier for $\mathrm{H}$ and 1 time interval earlier for $\mathrm{E}$.

$$
\widetilde{E}_{z}(i, j, n+0.5)=\widetilde{E}_{z}(i, j, n-0.5)+\frac{\Delta t}{\Delta x \sqrt{\varepsilon_{0} \mu_{0}}}\left[H_{y}(i+0.5, j, n)-H_{y}(i-0.5, j, n)-\left(H_{x}(i, j+0.5, n)-H_{y}(i, j+0.5, n)\right)\right]
$$

The $\left(\mathrm{H}_{\mathrm{x}}\right)$ and $\left(\mathrm{H}_{\mathbf{y}}\right)$ components can be discretized in space and time as:

$$
\begin{aligned}
& H_{x}(i, j+0.5, n+1)=H_{x}(i, j+0.5, n)-\frac{\Delta t}{\Delta x \sqrt{\varepsilon_{0} \mu_{0}}}\left[\widetilde{E}_{z}(i, j+1, n+0.5)-\widetilde{E}_{z}(i, j, n+0.5)\right] \\
& H_{y}(i+0.5, j, n+1)=H_{y}(i+0.5, j, n)+\frac{\Delta t}{\Delta x \sqrt{\varepsilon_{0} \mu_{0}}}\left[\widetilde{E}_{z}(i+1, j, n+0.5)-\widetilde{E}_{z}(i, j, n+0.5)\right]
\end{aligned}
$$

Equations (3-52), (3-53) and (3-54) all contain the constant $\frac{\Delta t}{\Delta x \sqrt{\varepsilon_{0} \mu_{0}}}$ known as the Courant Factor. It has been shown by many authors of FDTD books ${ }^{4}$ that stability is 
ensured when this constant is set less than or equal to 0.5 . This implies that once we discretized the computational spatial domain into grids of spacing $\Delta x$ we can determine the time interval $\Delta t$ that ensures stability, $\Delta t=\frac{\Delta x}{2 \sqrt{\varepsilon_{0} \mu_{0}}}$.

\subsubsection{Perfectly Matched Layer (PML):}

To limit the computational region the edge effects must be considered. They should absorb the out-going EM field by suppressing the spurious back reflected fields regardless of the polarization, propagation direction and frequency. The most common form of absorbing boundary condition used in FDTD simulation is the perfectly matched layer (PML) ${ }^{4}$, which may be placed about the periphery of the computation grid ${ }^{57}$. The computational domain is surrounded by a lossy material that absorbs the unwanted reflected energy such that the field is decaying exponentially inside the PML region. The PML is easy in concept:

i. Match impedances between grid points at the boundary;

ii. Increase the absorption as the wave propagates further into the PLM layer.

A small mathematical model saves system resources and speeds up the computational process. To match the impedances, the reflection coefficient, $\Gamma$ should be made equal to zero as:

$$
\Gamma=\frac{\eta_{A}-\eta_{B}}{\eta_{A}+\eta_{B}}=0
$$


To achieve this $\eta_{A}=\eta_{B}$, the impedance in the PML equals the impedance in the background medium of the computational grid. The PML is characterized by fictitious permittivities, $\varepsilon_{F m}^{*}(m)$, and permeabilities, $\mu_{F m}^{*}(m)$, in each of the propagation directions, $m=(x, y, z)$; then, impedance matching requires that:

$$
\sqrt{\frac{\varepsilon_{\mathrm{Pm}}^{*}}{\mu_{\mathrm{Fm}}^{*}}}=1
$$

The other condition to satisfy is that the fictitious permittivity and permeability in one direction must be the inverse of those in the other direction, when considering the direction normal to the boundary and the directions in the plane of the boundary. For 2-D TM analysis in the (X, Y) plane, the permittivities and permeabilities may be written in complex form as:

$$
\varepsilon_{\mathrm{Fm}}^{*}=\varepsilon_{\mathrm{Fm}}+\frac{\sigma_{\mathrm{Em}}}{j \omega \mu_{0}} \quad \text { for }(\mathrm{m}=\mathrm{x} \text { or } \mathrm{y})
$$

where, $\varepsilon_{\mathrm{Fm}}^{*}=$ permittivity of PML $; \varepsilon_{\mathrm{Fm}}=$ permittivity of background, and:

$$
\mu_{\mathrm{Fm}}^{*}=\mu_{\mathrm{Fm}}+\frac{\sigma_{\mathrm{Hm}}}{j \omega \mu_{0}} \quad \text { for }(\mathrm{m}=\mathrm{x} \text { or } \mathrm{y})
$$

where, $\mu_{\mathrm{Fm}}^{*}=$ permeability of PML; $\mu_{\mathrm{Fm}}=$ permeability of background; $\sigma_{\mathrm{Em}}=\sigma_{\mathrm{Hm}}=$ material conductivity. 
After introducing PML, equations (3-48), (3-49) and (3-50) for TM polarization consisting of field components $\tilde{E}_{z}$ and $\left(H_{x}, H_{y}\right)$ can be recast as:

$\widetilde{E}_{z}(i, j, n+0.5)$

$$
\begin{aligned}
& =g i 3(i) \cdot g j 3(j) \cdot \widetilde{E}_{z}(i, j, n-0.5)+\frac{\Delta t}{\Delta x \sqrt{\varepsilon_{0} \mu_{0}}} \cdot g i 2(i) \cdot g j 2(j) \\
& \cdot\left[H_{y}(i+0.5, j, n)-H_{y}(i-0.5, j, n)\right. \\
& \left.-\left(H_{x}(i, j+0.5, n)-H_{y}(i, j-0.5, n)\right)\right]
\end{aligned}
$$

$$
\text { where, } \begin{aligned}
g i 3(i) & =\left(\frac{1-\frac{\sigma_{\mathrm{p}}(\mathrm{i}) \Delta t}{2 \varepsilon_{0}}}{1+\frac{\sigma_{\mathrm{D}}(1) \Delta \mathrm{t}}{2 \varepsilon_{0}}}\right) ; g j 3(j)=\left(\frac{1-\frac{\sigma_{\mathrm{p}}(j) \Delta t}{2 \varepsilon_{0}}}{1+\frac{\sigma_{\mathrm{D}}() \Delta \mathrm{t}}{2 \varepsilon_{0}}}\right) ; \\
\operatorname{gi} 2(\mathrm{i}) & =\left(\frac{1}{1+\frac{\sigma_{\mathrm{D}}(1) \Delta \mathrm{t}}{2 \varepsilon_{0}}}\right) ; g j 2(j)=\left(\frac{1}{1+\frac{\sigma_{\mathrm{D}}(1) \Delta \mathrm{t}}{2 \varepsilon_{0}}}\right)
\end{aligned}
$$

$$
\begin{aligned}
\mathrm{H}_{\mathrm{x}}(\mathrm{i}, \mathrm{j}+0.5, \mathrm{n}+1) \\
\quad=f j 3(\mathrm{j}+0.5) \cdot \mathrm{H}_{\mathrm{x}}(\mathrm{i}, \mathrm{j}+0.5, \mathrm{n})-f j 2(j \\
\quad+0.5)\left(\frac{\Delta \mathrm{t}}{\Delta \mathrm{x} \sqrt{\varepsilon_{0} \mu_{0}}} \operatorname{curl}_{\mathrm{j}}\left(\widetilde{\mathrm{E}}_{\mathrm{z}}(\mathrm{n}+0.5)\right)\right. \\
\left.+\operatorname{gi1}(\mathrm{i}) \sum_{\mathrm{f}=0}^{\mathrm{n}} \operatorname{curl}_{\mathrm{j}}\left(\widetilde{\mathrm{E}}_{\mathrm{z}}(\mathrm{f}+0.5)\right)\right)
\end{aligned}
$$

where, $f j 3(j+0.5)=\left(\frac{1-\frac{\sigma_{D}(j+0.5) \Delta t}{2 \varepsilon_{0}}}{1+\frac{\sigma_{D}(j+0.5) \Delta t}{2 \varepsilon_{0}}}\right) ; f j 2(j+0.5)=\left(\frac{1}{1+\frac{\sigma_{D}(j+0.5) \Delta t}{2 \varepsilon_{0}}}\right)$ 


$$
g i 1(i)=\frac{\sigma_{\mathrm{D}}(\mathrm{i}) \Delta \mathrm{t}}{\varepsilon_{0}} \frac{\Delta \mathrm{t}}{\Delta \mathrm{x} \sqrt{\varepsilon_{0} \mu_{0}}}
$$

$$
\begin{aligned}
H_{y}(i+0.5, j, n & +1) \\
= & f i 3(i+0.5) \cdot H_{y}(i+0.5, j, n)+f i 2(i+0.5) \\
& \cdot\left[\frac{\Delta t}{\Delta x \sqrt{\varepsilon_{0} \mu_{0}}} \operatorname{curl}_{i}\left(\widetilde{E}_{z}(n+0.5)\right)+g j 1(j) \sum_{f=0}^{n} \operatorname{curl}_{i}\left[\widetilde{E}_{z}(f+0.5)\right]\right]
\end{aligned}
$$

$$
\begin{gathered}
\text { Where, } f i 3(\mathrm{i}+0.5)=\left(\frac{1-\frac{\sigma_{\mathrm{D}}(i+0.5) \Delta t}{2 \varepsilon_{0}}}{1+\frac{\sigma_{\mathrm{D}}(1+0.5) \Delta t}{2 \varepsilon_{0}}}\right) ; f i 2(\mathrm{i}+0.5)=\left(\frac{1}{1+\frac{\sigma_{\mathrm{D}}(1+0.5) \Delta \mathrm{t}}{2 \varepsilon_{0}}}\right) \\
g j 1(j)=\frac{\sigma_{\mathrm{D}}(\mathrm{j}) \Delta \mathrm{t}}{\varepsilon_{0}} \frac{\Delta \mathrm{t}}{\Delta x \sqrt{\varepsilon_{0} \mu_{0}}}
\end{gathered}
$$

\subsubsection{Transmission Spectrum:}

The optical properties of a photonic crystal can be determined by a transmission spectrum obtained with the help of FDTD analysis. The transmission spectrum indicates the wavelengths that can propagate through the photonic crystal structure and also show bandgap regions where certain wavelengths are excluded. Figure 3.8 shows the optical layout in the FDTD simulator used to obtain the transmission spectra of the 2-D photonic crystal with an array of triangular lattices (TM polarization). This triangular array contains 39 periods in the $\mathrm{X}$ direction and 15 periods in the $\mathrm{Y}$ direction, having overall dimensions of $10 \mu \mathrm{m} * 25 \mu \mathrm{m}$ with 150 points per micron. The width of the PML is 
considered as $2 \mu \mathrm{m}$. The source line on the left side produced a short pulse of $4 \mathrm{fs}$ in duration, such that the waves propagate from left to the right towards the detection point (FFT point).

The short pulse duration provides a suitable wavelength, $\lambda=0.8 \mu \mathrm{m}$ to $2.5 \mu \mathrm{m}$. The transform point records the time variation of all the fields. The Fourier transform of this variation is given by:

$$
E(f)=\sum_{n=0}^{T_{n}} E(n \Delta t) e^{-j 2 \pi f \Delta t}
$$

where, $T_{n}$ is the number of time iterations and $\Delta t$ is the time increment per iteration. Using $c=\lambda f$, the field amplitude as a function of wavelength is obtained.

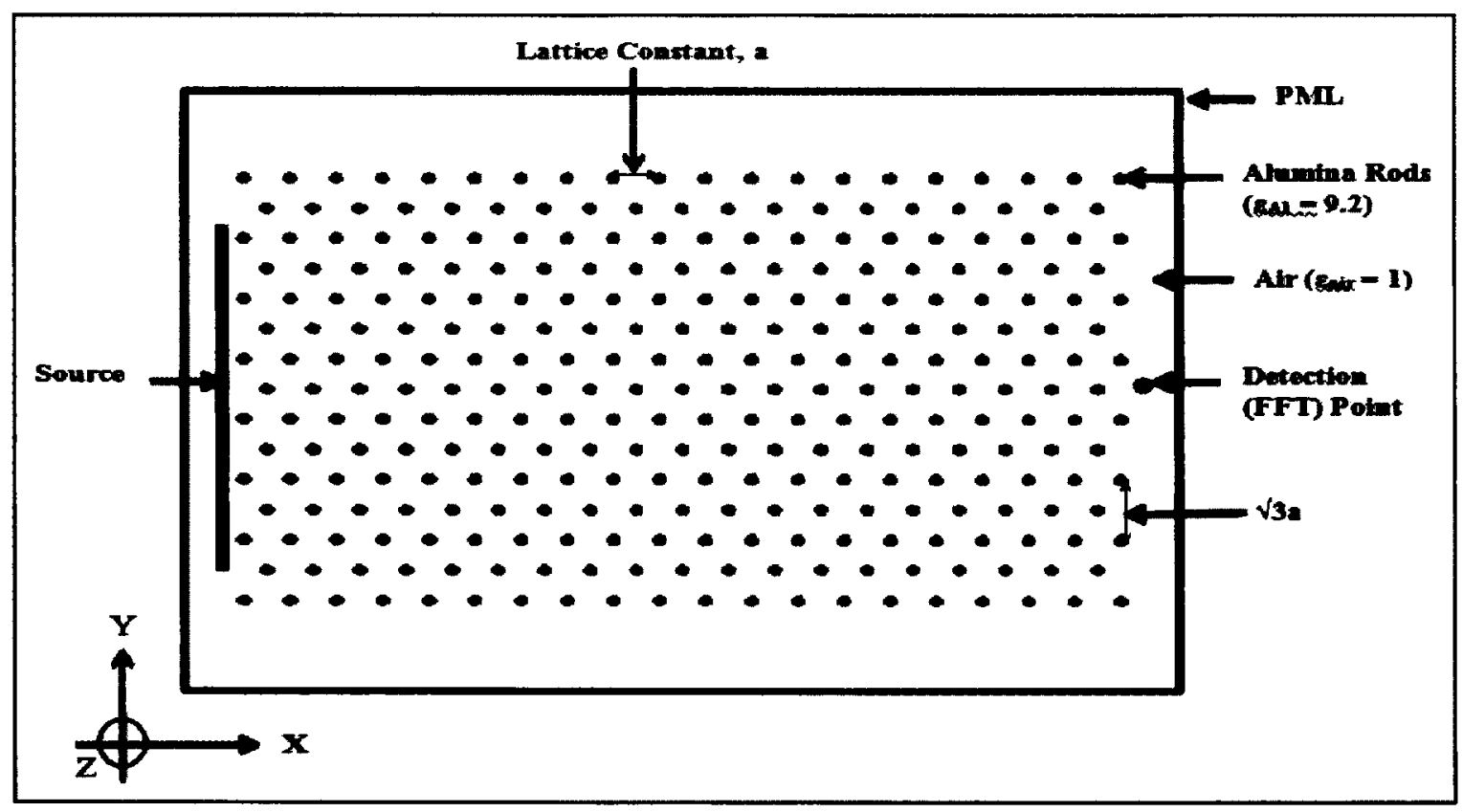

Figure 3.8: A 2-D triangular lattice photonic crystal place in the FDTD environment in order to determine the transmission spectrum. Alumina rod (black dots), radius, $r=0.1 \mu \mathrm{m}$ with dielectric constant, $\left(\varepsilon_{\mathrm{Al}}=9.2\right)$ with a background (white) as air $\left(\varepsilon_{\text {air }}=1\right.$ ) and lattice constant, $a=0.70 \mu \mathrm{m}$. 
Figure 3.9 shows the transmission spectrum of the 2-D photonic crystal with an array of alumina rod arranged in a triangular lattice (TM polarization). After analyzing this spectrum, there is an existence of photonic bandgap from $1.28 \mu \mathrm{m}-2.02 \mu \mathrm{m}$, which is in good agreement with plane wave expansion results as shown in Figure 3.3 and Figure 3.4.

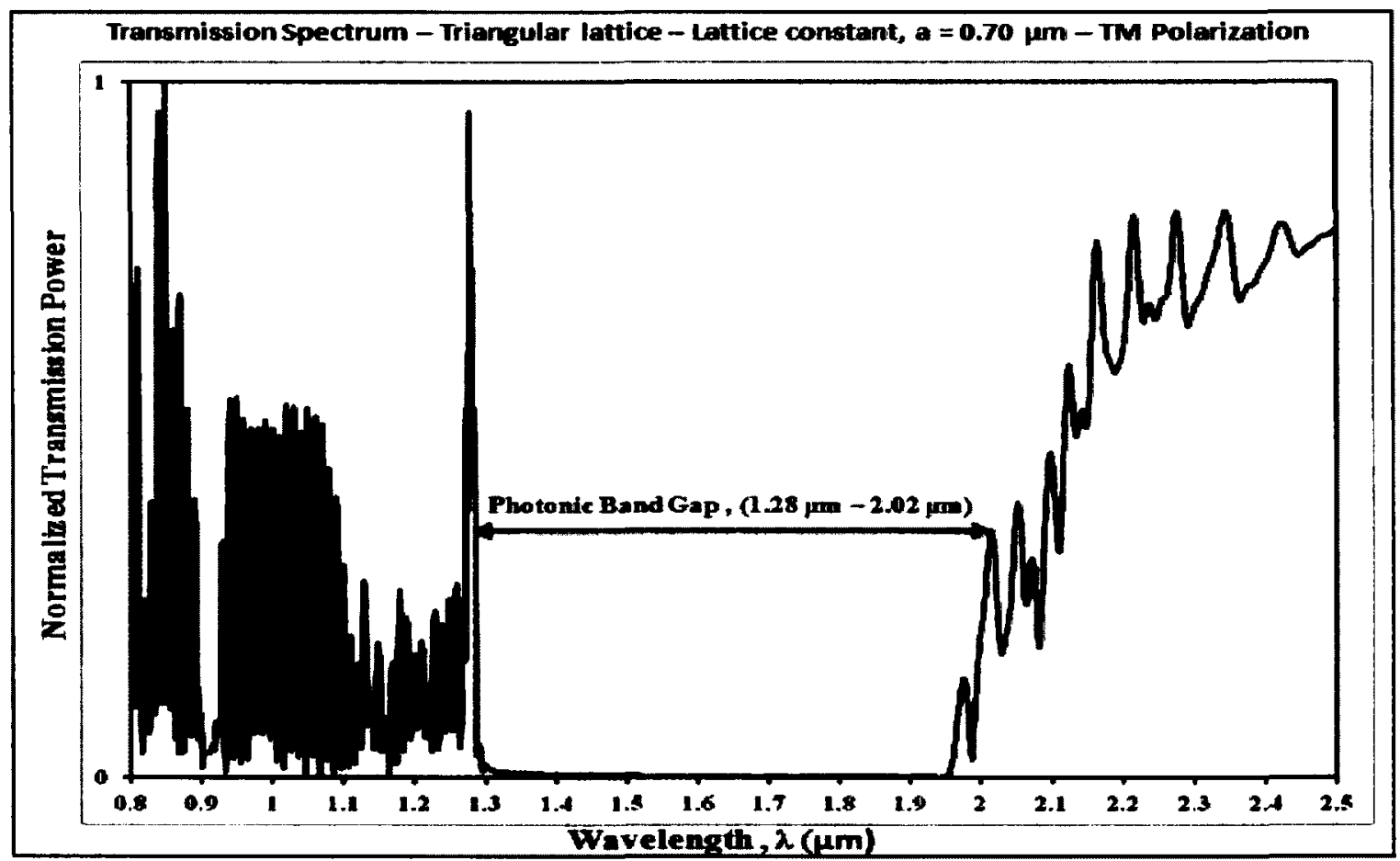

Figure 3.9: Transmission spectrum obtained from the FDTD simulator for a triangular array photonic crystal (TM polarization) after 100,000 iterations. A bandgap obtained at wavelength range of, $\lambda=1.28 \mu \mathrm{m}$ to $2.02 \mu \mathrm{m}$.

Figure 3.10 shows the optical layout in the FDTD simulator used to obtain the transmission spectra of the 2-D linear waveguide with an array of triangular lattices (TM polarization). This triangular array contains a total of 39 periods in the $\mathrm{X}$ plane and 15 periods in the $\mathrm{Y}$ direction with the center row $\left(8^{\text {th }}\right)$ of alumina rods removed. The source 
line on the left side produced a short pulse, such that the waves propagate from left to right towards the detection point (FFT point). The transform point records the time variation of all fields and this results in the formation of the transmission spectrum.

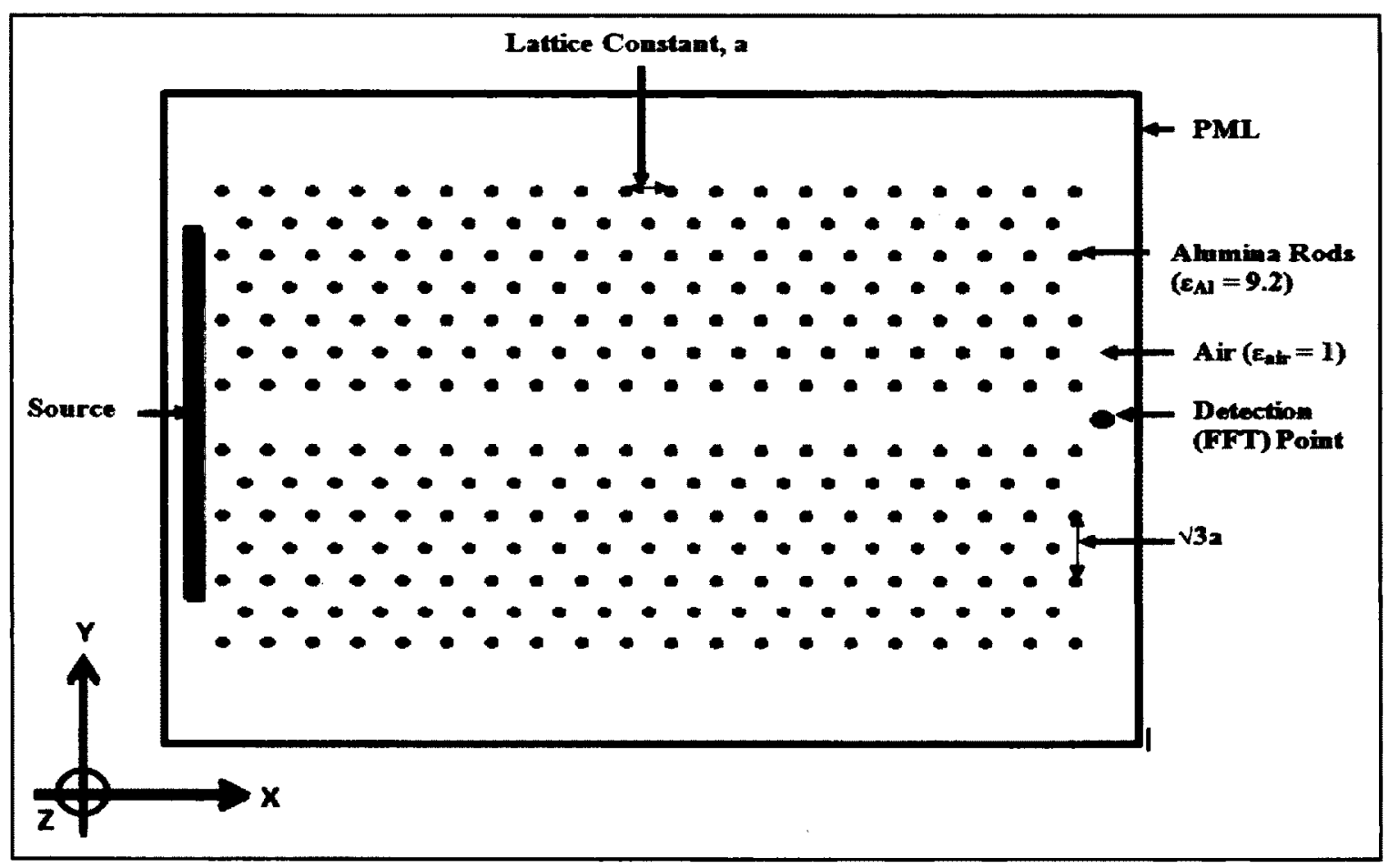

Figure 3.10: A 2-D linear waveguide formed by removing the $8^{\text {th }}$ row of alumina rods in the triangular lattice. Alumina rod (black dots), radius, $\mathrm{r}=0.1 \mu \mathrm{m}$ with dielectric constant, $\left(\varepsilon_{\mathrm{Al}}=9.2\right)$ with a background (white) as air $\left(\varepsilon_{\mathrm{air}}=1\right)$ and lattice constant, $\mathrm{a}=0.70 \mu \mathrm{m}$.

Figure 3.11 shows the transmission spectrum of the 2-D photonic crystal waveguide with an array of alumina rods arranged in a triangular lattice (TM polarization). After analyzing this spectrum, there is a certain range of wavelength, $\lambda=$ $1.35 \mu \mathrm{m}$ to $1.85 \mu \mathrm{m}$ that is getting through this waveguide channel that shows excellent agreement with the results obtained using the supercell technique (plane wave method) (refer to Figure 3.6). 


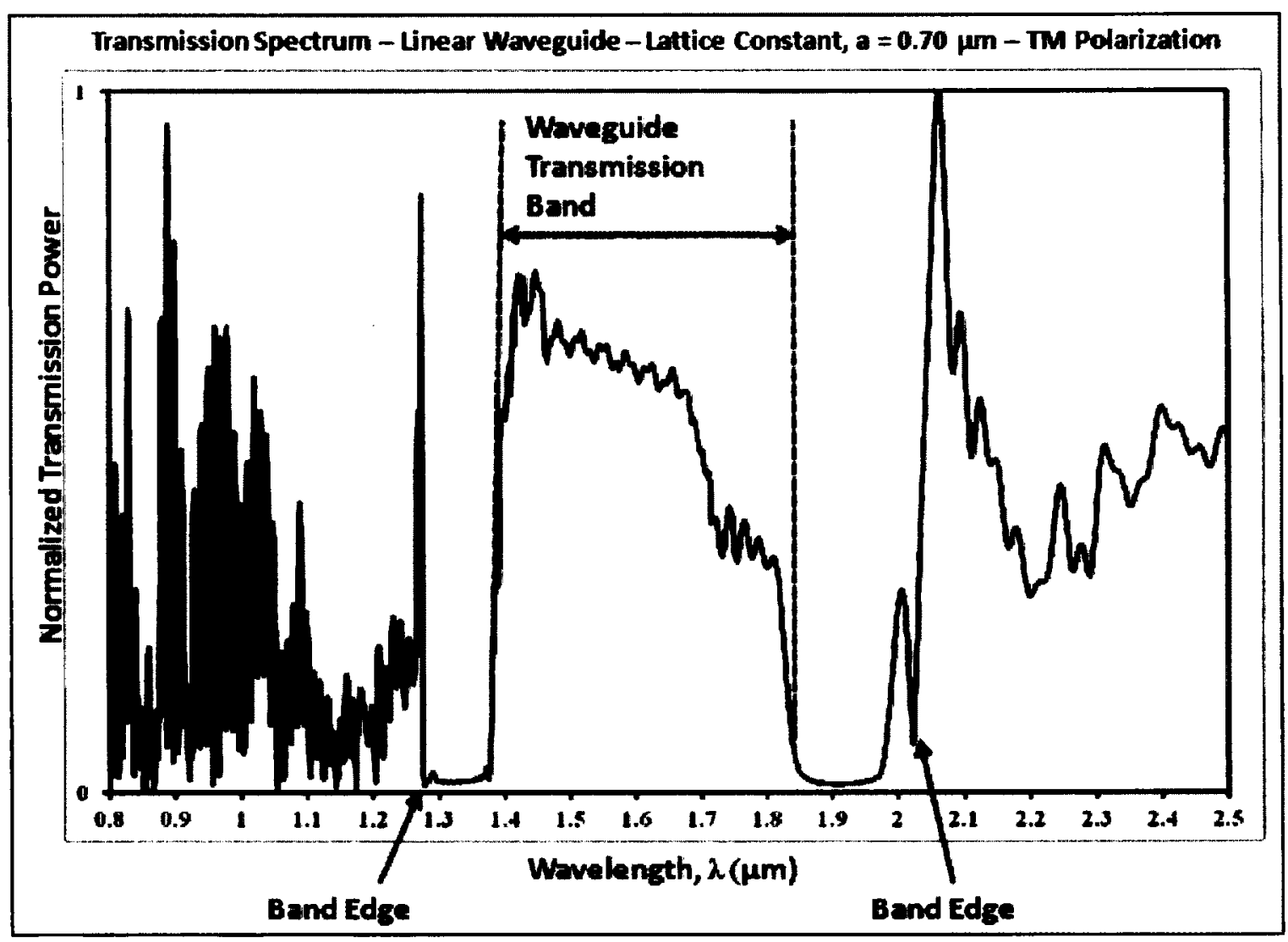

Figure 3.11: Transmission spectrum for a 2-D linear waveguide with triangular lattice obtained from the FDTD simulator after 100,000 iterations (TM polarization). The spectrum shows the presence of high transmission within the original bandgap region indicating that one or more modes can be propagated.

Now, PWM and FDTD results are satisfying the photonic bandgap. FDTD results also matches the supercell results showing a certain range of wavelength getting through the linear waveguide. It is good enough to set a microwave experimental demonstration to verify these simulation results.

In the following chapter, a 2-D photonic crystal linear waveguide with a triangular lattice is designed, and fabricated in a microwave regime to perform various experimental measurements. The scalability law is implemented for the conversion of the parameters from the optical to the microwave regime. 


\section{Experimental Setup}

In this chapter, the microwave experimental setup will be discussed with a short description of the various components and material used during the measurements, such as network analysis, horn antenna, alumina rod specifications and triangular lattice template design.

The scalability laws suggest that a linear electromagnetic system will have the same characteristics (except scaling factor, ' $s$ ') if the geometry is scaled. Using this property of scalability, many experimental demonstrations have been conducted and tested on photonic crystals with different dimensionalities (1D, 2D, 3D) and different defect formations (line/point) with either E-Field or H-Field or both polarizations at microwave frequencies of the electromagnetic spectrum ${ }^{9}, 14,15,17,20,25,28,27,28,41,42,45,46,55$.

A very common type of symmetry used for the 2-D photonic crystal is a triangular lattice that shows a large bandgap and has a substantial advantage in terms of compactness, stability and fabrication.

\subsection{Vector Network Analyzer (VNA)}

An HP 8720 ES S-parameter Vector Network Analyzer (VNA) - $(50 \mathrm{MHz}-20 \mathrm{GHz})$ was used for all microwave experimental measurements. The VNA measures the magnitude and phase characteristics of various components, such as amplifiers, splitters, filters, attenuators, cables, and antennas. It compares the incident signal that leaves the analyzer with either the signal that is transmitted through the test device or the signal that is reflected back into the input. Two types of measurements are carried out by the 
network analyzer, namely transmission measurements and reflection measurements. Figure 4.1 (a) and (b) describes the type of measurements (transmission and reflection) that can be made and the inset shows a pictorial view of the VNA.

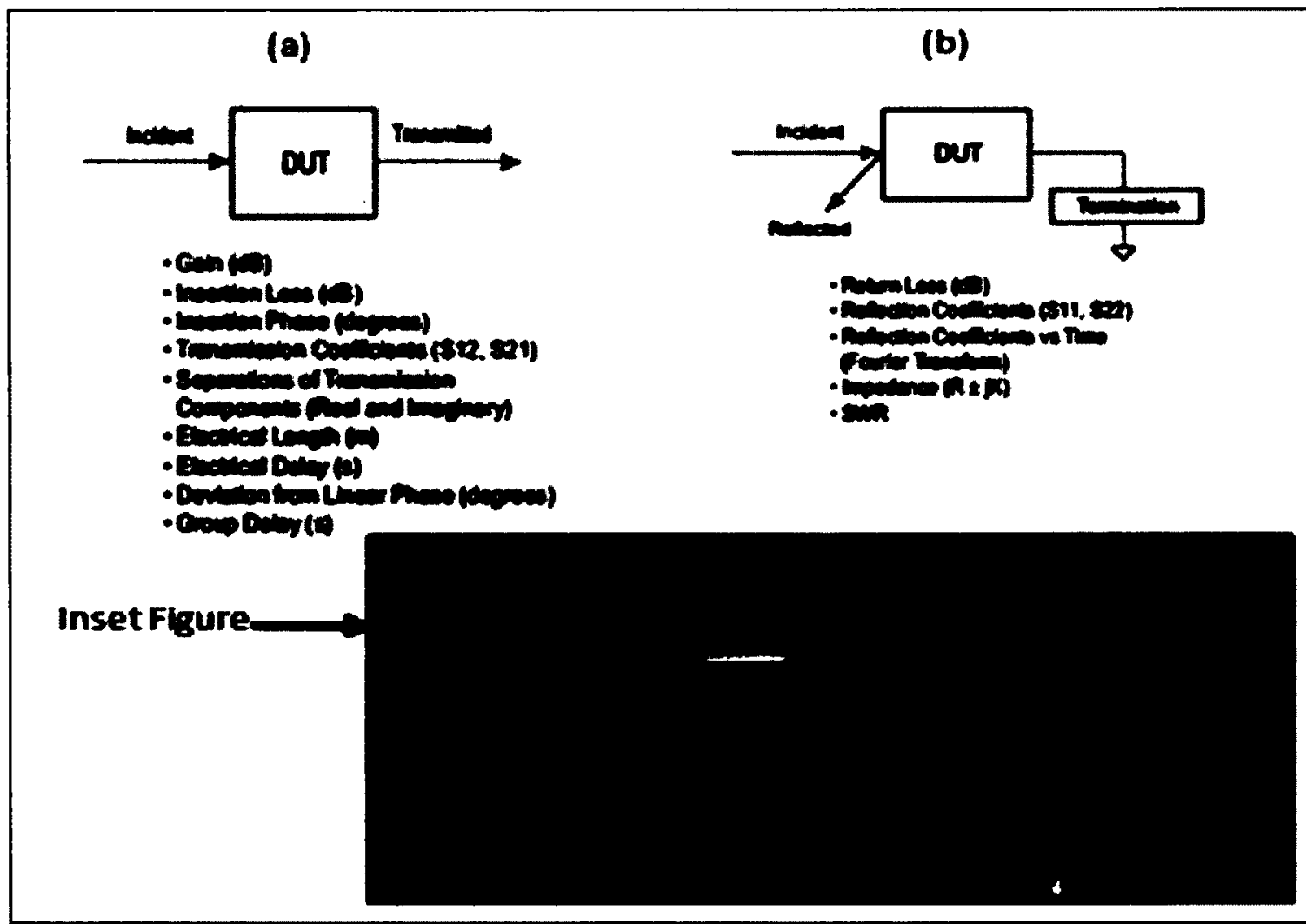

Figure 4.1: Measurements performed with the VNA: (a) transmission measurements; (b) reflection measurements; the inset shows the pictorial view of the HP 8720 ES S-parameter Vector Network Analyzer $-(50 \mathrm{MHz}-20 \mathrm{GHz})$

In a two-port network, transmission measurements include various parameters, such as insertion loss, insertion phase, transmission coefficients, electrical delays, and group delay that help to determine the electrical behavior of particular devices under testing. Similarly, reflection measurements include return loss, reflection coefficient, 
impedance, and standing wave ratio (SWR) that also determine the electrical behavior of the device under testing. The S-parameters play an important role to determine various properties of the device under testing. Figure 4.2 illustrates the four S-parameters for a two-port network as:

- $\mathrm{S}_{11}-$ Forward reflection (power reflected from port 1 to port 1)

- $S_{21}-$ Forward transmission (power transmitted from port 1 to port 2)

- $\mathrm{S}_{22}-$ Reverse reflection (power transmitted from port 2 to port 2)

- $\mathrm{S}_{12}-$ Reverse transmission (power reflected from port 2 to port 1)

\section{S21 Forward Transmission}

S11 Forward Reflection Port 2

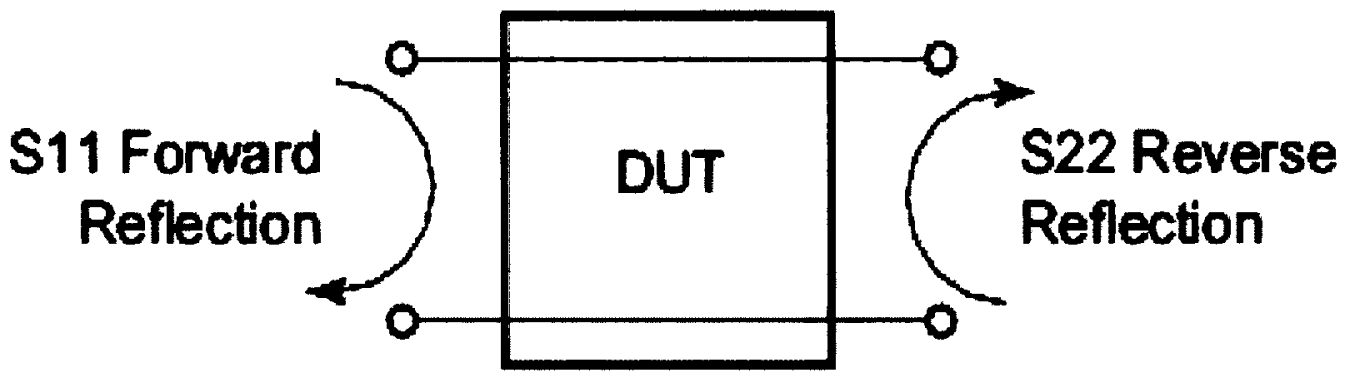

S12 Reverse Transmission

Figure 4.2: Two-port S-parameters $\left(S_{11}, S_{21}, S_{22}, S_{12}\right)$ for the Vector Network Analyzer (VNA) 
In this thesis, only the forward transmission coefficient $\left(\mathbf{S}_{21}\right)$ was measured while performing microwave measurements on a 2-D photonic crystal model. This parameter helps to create a transmission spectrum between transmitted power $(\mathrm{dB})$ and frequency $(\mathrm{GHz})$ that shows the transmission properties of a device under testing at different frequencies.

\subsubsection{VNA Calibration Procedure:}

A full two-port calibration was done as per the instructions provided by the manufacturer ${ }^{58}$ to remove various errors, such as directivity error, source match error, and isolation error, both in forward and in reverse measurements. For every microwave measurement, the following calibration procedure was followed:

i. Turn on network analyzer and set screen display for four $\mathrm{S}$-parameters.

ii. After waiting for two hours, start the calibration process with the help of a calibration kit provided by the manufacturer.

iii. Set start $(2 \mathrm{GHz})$ and stop $(14 \mathrm{GHz})$ frequencies to get expected photonic bandgaps at around the frequency range of $8-9 \mathrm{GHz}$.

iv. Set the number of sweep points to 401 . The sweeping mode is an initial cycling between the test ports; then, the measurement stays on the active port for a user defined number of sweeps. After the specified number of sweeps has been executed, the analyzer switches between the test ports and begins the cycle again.

v. On the front panel of the network analyzer, select the calibration menu with full two-port. 
vi. Select reflection from the menu bar and connect a shielded open standard to port 1 as shown in Figure 4.3.

vii. To measure the open standard, when the displayed trace has settled, press 'forward:open'. The analyzer will take a few seconds to measure the standard and then underline the 'OPEN' softkey.

viii. Disconnect the open standard and connect a short standard to port 1 as shown in Figure 4.3.

ix. Wait for the display to settle and then press 'forward:short'. The analyzer will take a few seconds to measure the standard and then underline the 'SHORT' softkey.

x. Disconnect the short standard and connect an impedance matched load to port 1 as shown in Figure 4.3.

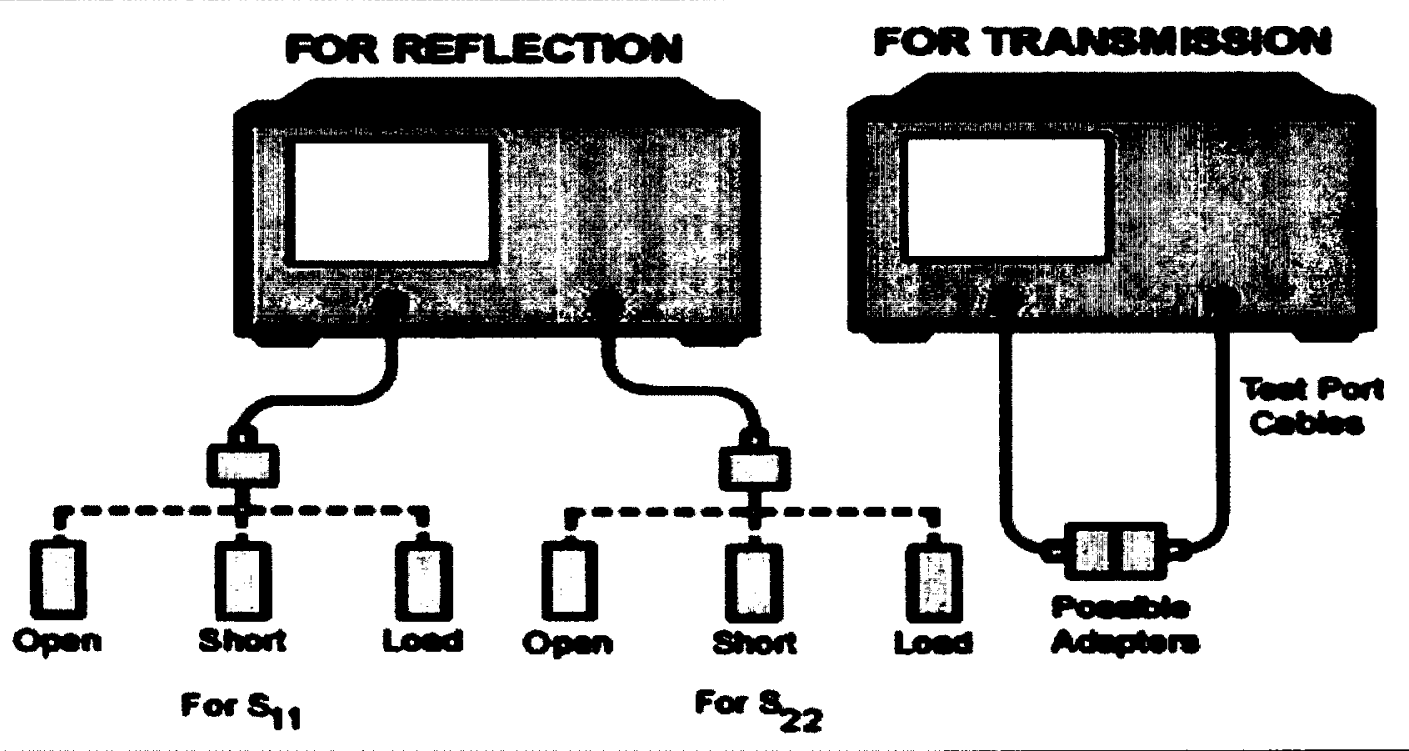

Figure 4.3: Calibration of full two-port network analyzer with standard connection as per the manufacturer's instructions. 
xi. Wait for the display trace to settle and press 'forward:load' and select the type of load (broadband); then press the 'done:loads' softkey. It will take a few seconds to measure the standard load and then underline the 'LOAD' softkey.

xii. Repeat steps (vii) to (xi) for port 2 and use 'reverse:open', 'reverse:short', 'reverse:load' softkeys.

xiii. To compute the reflection correction coefficients, press 'standards done' on the network analyzer panel.

xiv. To start the transmission portion of the correction, press 'transmission' on the control panel of network analyzer.

xv. Make a 'thru' connection between the two points (port 1 and port 2) where two horn antennas will be connected and a device under testing (triangular lattice array template) will be placed in between.

xvi. Wait for the display trace to settle and press, 'DO BOTH FWD+REV'. It will take a few seconds to make each measurement and an underline exists for the softkey label.

xvii. Press 'ISOLATION' and select 'OMIT ISOLATION' as the device under testing will be measured with a dynamic range less than $90 \mathrm{~dB}$.

xviii. To compute the error coefficients, press 'DONE 2-PORT CAL'.

xix. Finally, the network analyzer displays the corrected measurement trace on the display screen. The network analyzer is remotely controlled over a general purpose interface bus (GPIB ) by a computer. Now, all four S-parameter $\left(\mathbf{S}_{11}, \mathbf{S}_{12}\right.$, $\left.S_{21}, S_{22}\right)$ traces will be displayed on the computer screen. 


\subsection{Horn Antenna}

Two horn antennas (H-1498) and (EM-6961) were used as a transmitter and a receiver for experimental purposes as shown in Figure 4.4. These antennas are a broadband device, covering the frequency range from $1 \mathrm{GHz}$ to $18 \mathrm{GHz}$. In spite of the different model numbers, their performance is the same after interchanging their positions with respect to the network analyzer while performing the experiments.

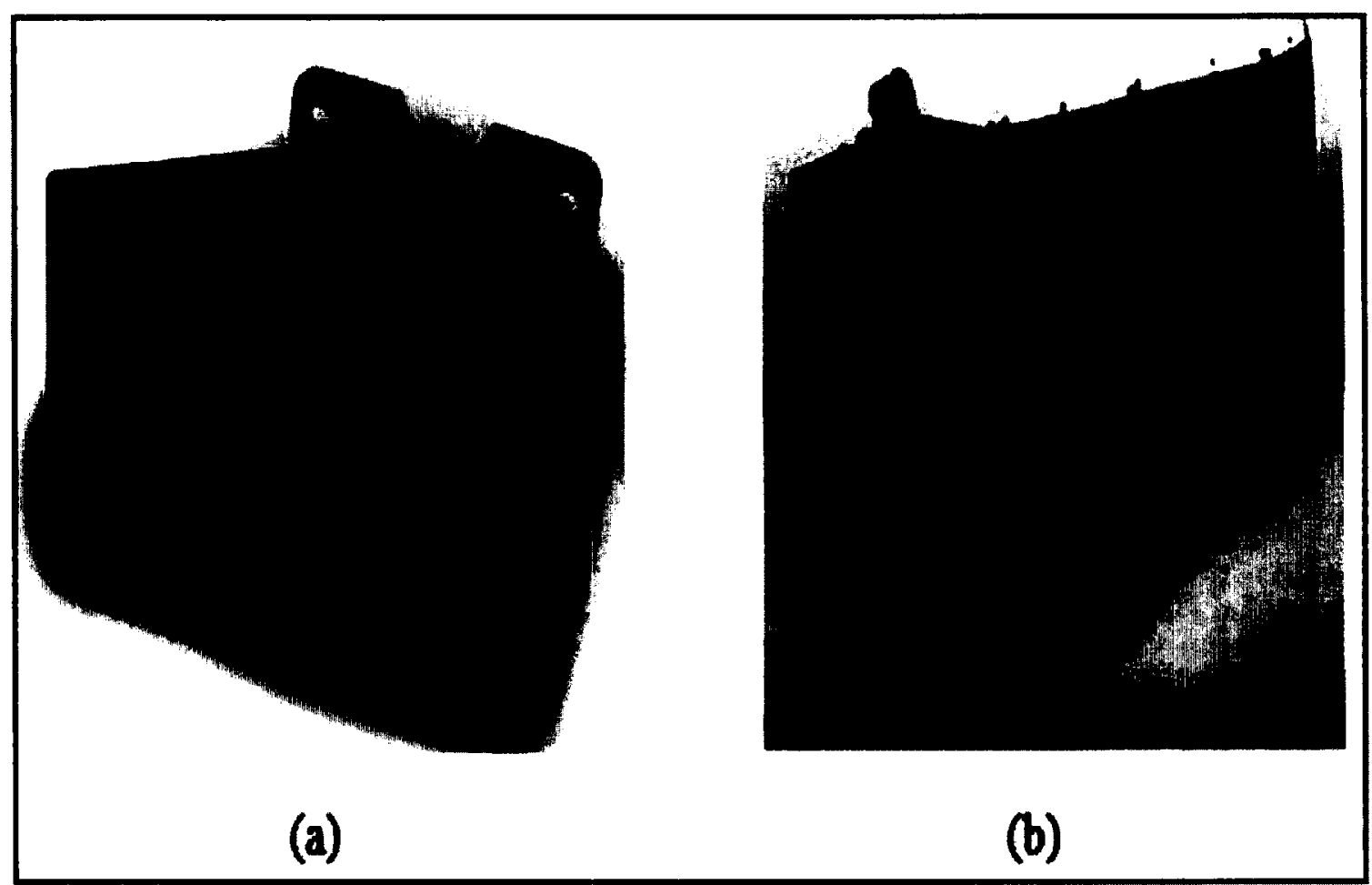

Figure 4.4: Pictorial view of two horn antennas (a) model H-1498 as receiver (b) model EM- 6961 as a transmitter used in the microwave experiments.

Specifications of the two horn antennas used as a transmitter and a receiver for the experiments are provided below in Table 4.1 


\begin{tabular}{|c|c|c|}
\hline Specifications & Model - H-1498 & Model - EM-6961 \\
\hline Description & $\begin{array}{c}\text { Double Ridged } \\
\text { Broadband Horn }\end{array}$ & $\begin{array}{c}\text { Double Ridged } \\
\text { Broadband Horn }\end{array}$ \\
\hline Frequency Range (GHz) & $2-18 \mathrm{GHz}$ & $2-18 \mathrm{GHz}$ \\
\hline Impedance (ohms) & 50 & 50 \\
\hline VSWR & $2.0: 1$ & $<1.5: 1$ \\
\hline Polarization & Linear & $10.7 \mathrm{dBi}$ \\
\hline Gain (referred to isotropic source) & $11 \mathrm{dBi}$ & $280 \times 244 \times 157$ \\
\hline Size (mm) & $99 \times 125 \times 81$ & \\
\hline
\end{tabular}

Table 4.1: Specifications of two horn antennas use for the microwave experiments.

\subsection{Microwave Measurement without a 2 D Photonic Crystal Model}

After calibration, transmission power $\left(\mathrm{S}_{21}\right)-(\mathrm{dB})$ was measured as a function of frequency $(\mathrm{GHz})$ between two horn antennas (transmitter and receiver) without placing a 2-D photonic crystal model in between them as shown in Figure 4.5. The distance between the transmitter and receiver was kept at $300 \mathrm{~mm}$. 


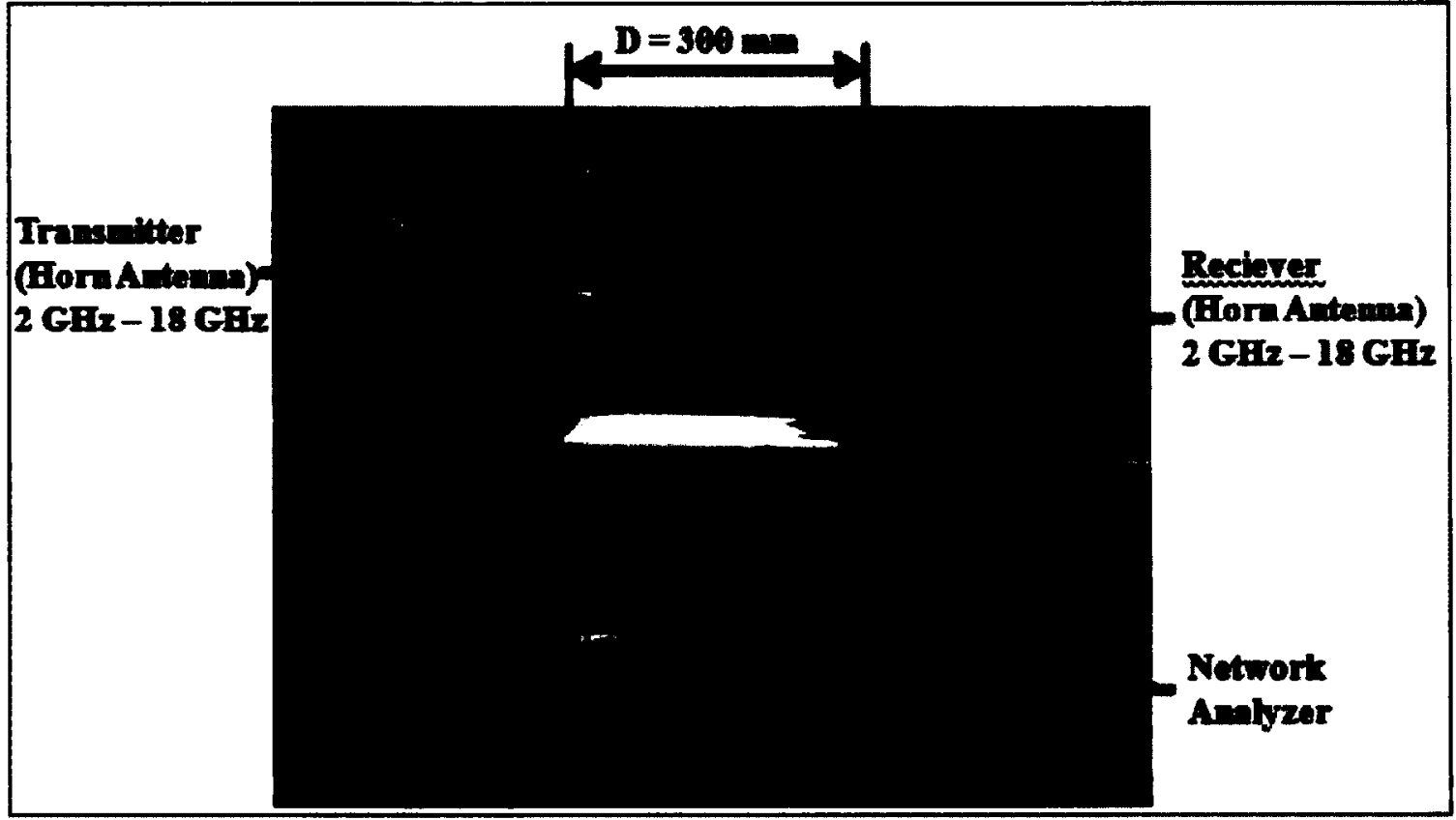

Figure 4.5: Two horn antennas (transmitter and receiver) with a distance of $300 \mathrm{~mm}$ was connected to a 2 port vector network analyzer with TM polarization and transmission power; $S_{21}(d B)$ was obtained without placing a -D photonic crystal model in between them.

All the raw data (S-parameters) are displayed and temporarily saved on a computer monitor with the help of GPIB or can be permanently saved on compact disk. A typical reference transmission curve is shown in Figure 4.6. The curve shows a slight rising part at the start and remains constant throughout the frequency range with an average reading of $-23 \mathrm{~dB}$. This is due to the reflection and interference caused by the surrounding area due to an inadequate presence of microwave absorption material. 


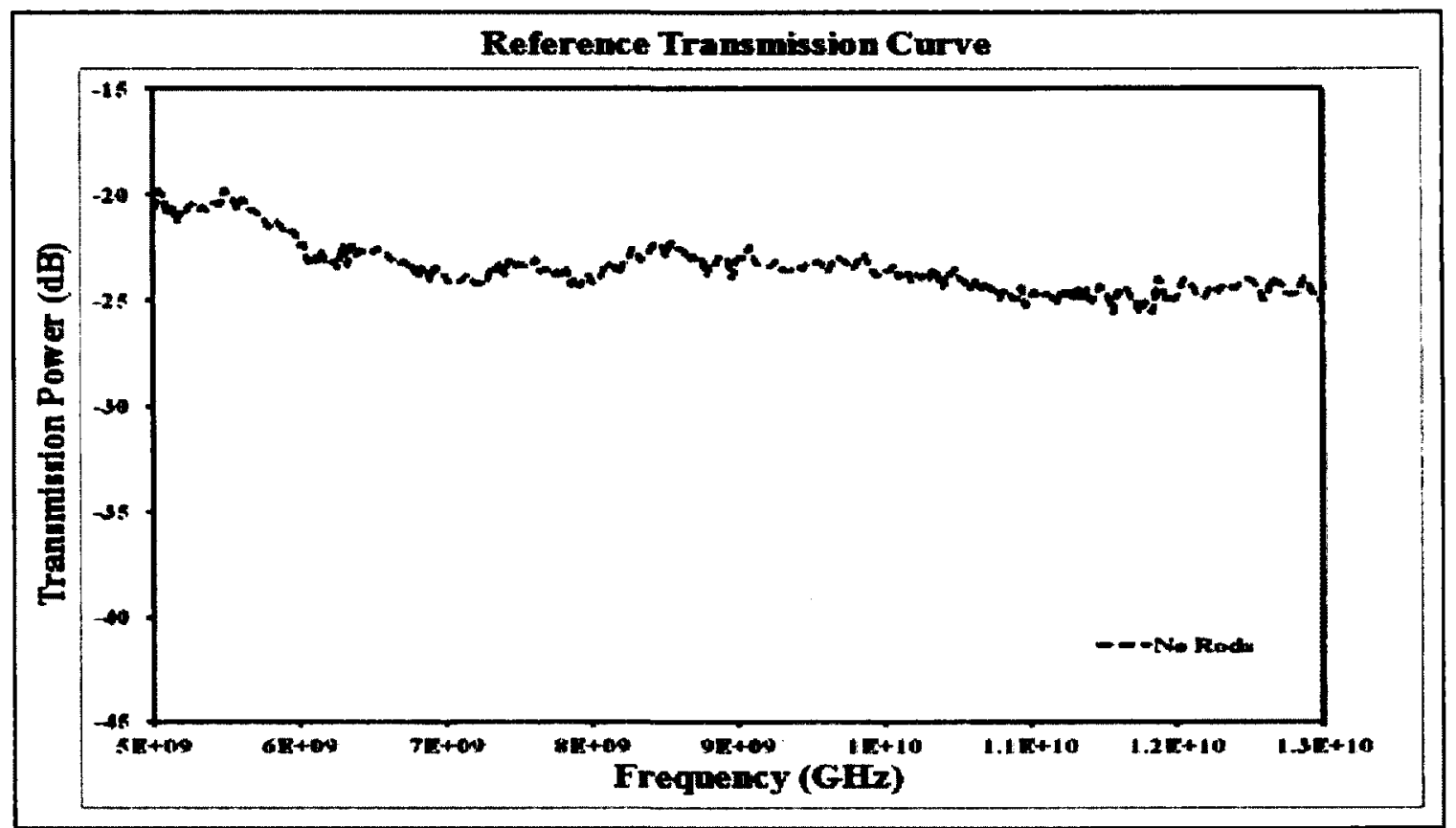

Figure 4.6: A reference transmission curve obtained when the transmitter and receiver (horn antennas) were placed at a distance of $300 \mathrm{~mm}$.

After obtaining a reference transmission curve, the measurement system is ready to analyze the microwave photonic crystal structures.

\subsection{Alumina Rod: Specifications and Characteristics}

Alumina rod specifications and characteristics are described in this section. A 2-D and 3D photonic crystal structure at a microwave frequency regime can be easily built with the availability of alumina rods having different shapes (circular, rectangular, etc.), size, diameter and refractive index. Various researchers $9,25,37,38,41,42,46,47$ performed many experimental demonstrations in the microwave frequency regime to confirm 2-D and 3-D photonic properties. 
Alumina is the most cost-effective and widely used material in the family of engineering fields. Some of the key properties of alumina material are:

i. Hardwearing and resistant

ii. Excellent dielectric properties from $\mathrm{DC}$ to $\mathrm{GHz}$ frequencies

iii. Resists strong acid and alkali attacks

iv. Good thermal conductivity

v. Excellent size and shape capability

vi. High strength and stiffness

vii. Available in purity ranges from $94 \%$ to $99.5 \%$ for the most demanding high temperature applications

With these key properties, alumina is used as a standard material for the manufacturing of hybrid structures ${ }^{48}$. The dielectric constant and dielectric loss of alumina were studied and experimentally verified at different frequencies in microwave applications $^{48,49}$.

For a microwave experimental demonstration, 300 circular alumina rods (AL 95, OD $0.188 " \pm 0.010 ", 12 \pm 0.062 "$ long, $95 \%$, alumina, Anderman Ceramics, USA) were used to verify simulation results obtained from the plane wave and FDTD methods. A brief data sheet provided by Superior Technical Ceramics Corp., an alliance partner of Anderman Ceramics, showing physical, thermal, mechanical and electrical properties is attached to Appendix B.

The outer diameter of each alumina rod was measured with the help of digital calliper and 1,500 readings were recorded. The rod diameter plays a vital role in 
confining the electromagnetic waves through the structure and evaluating wave vector $\vec{k}$ while plotting band structures. The rod diameter was considered for the measurements because the length of the rods is considered infinite, as in a case of a 2-D photonic crystal, and the dielectric constant fluctuation produces small variations in bandgaps. A statistical analysis was performed on the collected data to get the final value of the rod diameter, i.e. $d=0.485 \mathrm{~cm} / 0.191$ inches as shown in Figure 4.7. A bell-shaped curve (Gaussian distribution) was created while doing statistical analysis on the collected data. From this curve, it was observed that there was a difference of $1.68 \%$ from the actual rod diameter provided by the manufacturer. All the measurements and analysis carried out throughout the work were restricted to three decimal places.

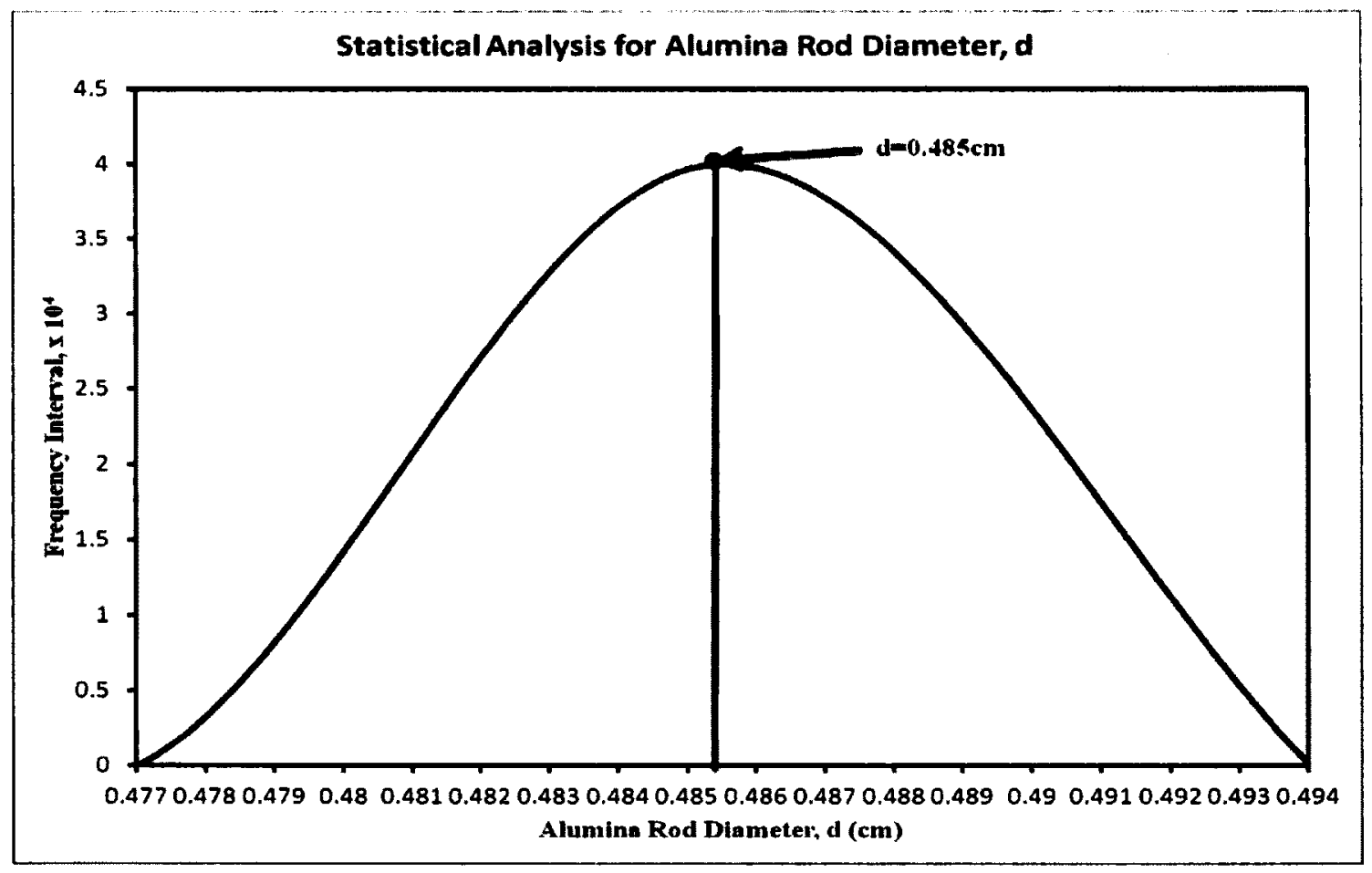

Figure 4.7: Statistical analysis (bell-shaped curve) of the measurements (1,500 readings) made on 300 circular alumina rods with the help of a digital calliper to get the final rod diameter, $\mathrm{d}=0.485 \mathrm{~cm}$. 
Having discussed the specifications and characteristics of the alumina rods, the design parameters for the 2-D photonic crystal model in the microwave regime will be discussed in the next section.

\subsection{Design Parameters for the 2-D Photonic Crystal model - (Microwave Regime):}

The law of scalability as stated in Chapter 2 was implemented for necessary conversion of the parameters from the optical to the microwave and vice versa. In this thesis, for the experimental (microwave regime) design of a 2-D photonic crystal, various parameters were considered as follows (see Table 4.2):

\begin{tabular}{|c|c|}
\hline Frequency range & $2 \mathrm{GHz}$ to $14 \mathrm{GHz}$ \\
\hline Lattice & Triangular \\
\hline Polarization & Transverse Magnetic (TM) \\
\hline Dielectric constant & $9.2( \pm 0.01)$ \\
\hline Alumina rod diameter, $d$ & $4.85 \mathrm{~mm}$ \\
\hline Lattice constant, $a$ & $16.9 \mathrm{~mm}$ \\
\hline Waveguide channel width, $\mathrm{W}$ & $24.45 \mathrm{~mm}$ \\
\hline Scaling factor, 's' & 24176.45 \\
\hline
\end{tabular}

Table 4.2: Parameters used for experimental design in the microwave regime. 
The next section will explain the 2-D photonic crystal template design in the microwave regime.

\subsection{2-D Photonic Crystal Template Design}

A 2-D photonic crystal template was designed and fabricated in the microwave regime using a plexi-glass sheet $(300 \mathrm{~mm} \mathrm{X} 763 \mathrm{~mm} \mathrm{X} 20 \mathrm{~mm})$ with holes drilled in a triangular array. The thickness of $20 \mathrm{~mm}$ was chosen so that alumina rods could fully fit into the holes in a vertical direction ( $\mathrm{Z}$ axis) without requiring any additional support. The plexiglass sheet was cut into three main sections and holes were drilled to accommodate up to 500 alumina rods with a diameter, $d=4.85 \mathrm{~mm}$ and a lattice constant, $a=16.9 \mathrm{~mm}$ as shown in Figure 4.8.

These three sections are:

i. Section 1 - Fixed Part: (17 periods in $\mathrm{Y}$ direction and 19 periods in $\mathrm{X}$ direction)

ii. Section 2 - Moving Part: (17 periods in $\mathrm{Y}$ direction and 32 periods in $\mathrm{X}$ direction)

iii. Section 3 - Fixed Part: (17 periods in $\mathrm{Y}$ direction and 11 periods in $\mathrm{X}$ direction)

To design a 2-D photonic crystal template with a triangular lattice for microwave experiments, out of 62 periods $(529.8 \mathrm{~mm})$, only 35 periods $(262.45 \mathrm{~mm})$ in the $X$ direction and all 17 periods $(239.1 \mathrm{~mm})$ in the $\mathrm{Y}$ direction were used to accommodate 300 alumina rods. A linear waveguide was formed manually with a channel width of $24.45 \mathrm{~mm}$ by removing the ninth row (removing 18 alumina rods) in the $\mathrm{X}$ direction. 


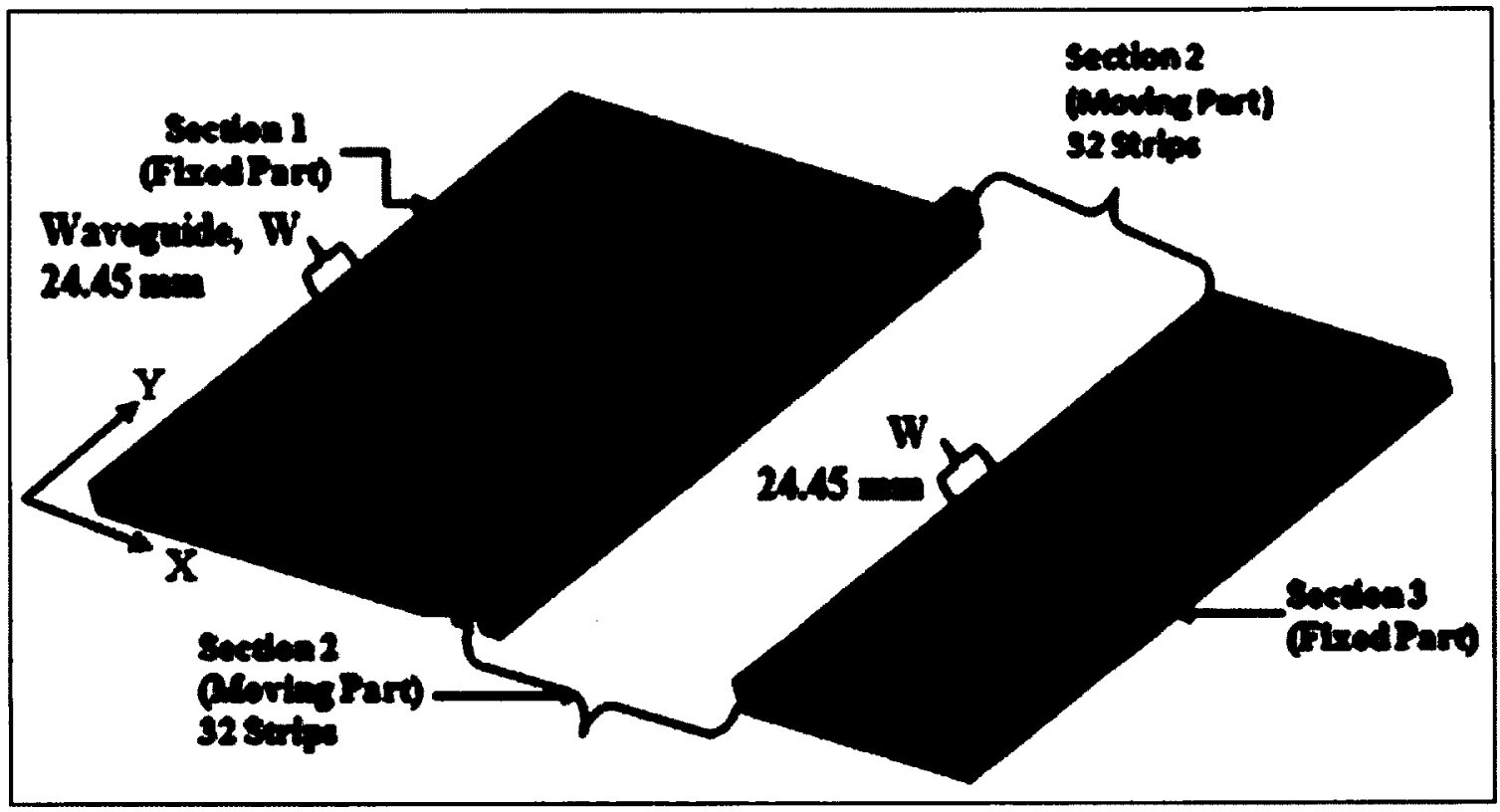

Figure 4.8: A 2-D photonic crystal template with a triangular lattice array consisting of three main sections with overall dimensions of $299.1 \mathrm{~mm} \mathrm{X} 569.8 \mathrm{~mm}$ having a total of 17 periods in the Y direction and 62 periods in the $\mathrm{X}$ direction. The linear waveguide is formed by removing the ninth row in the $\mathrm{X}$ direction, the waveguide channel width, $\mathrm{W}=24.45 \mathrm{~mm}$.

\section{i. Section 1 - Fixed Part:}

The overall dimensions of this plexi-glass section are $299.1 \mathrm{~mm} \mathrm{X} 178.7 \mathrm{~mm}$. This section contains 17 periods $(239.1 \mathrm{~mm})$ in the $\mathrm{Y}$ direction and 19 periods $(158.7 \mathrm{~mm})$ in the $\mathrm{X}$ direction to accommodate up to 152 alumina rods as shown in Figure 4.9. A transmitter (hom antenna) was placed at the starting edge of this section to transmit electromagnetic waves through the 2-D structure. To construct a 2-D photonic crystal structure and perform an experimental measurement, 17 periods in the $\mathrm{Y}$ direction and only 9 periods in the $\mathrm{X}$ direction were considered to accommodate 77 alumina rods (72 
with the linear waveguide). This section was kept fixed along with the transmitter (horn antenna), so that it remained in the same position throughout the experimental process.

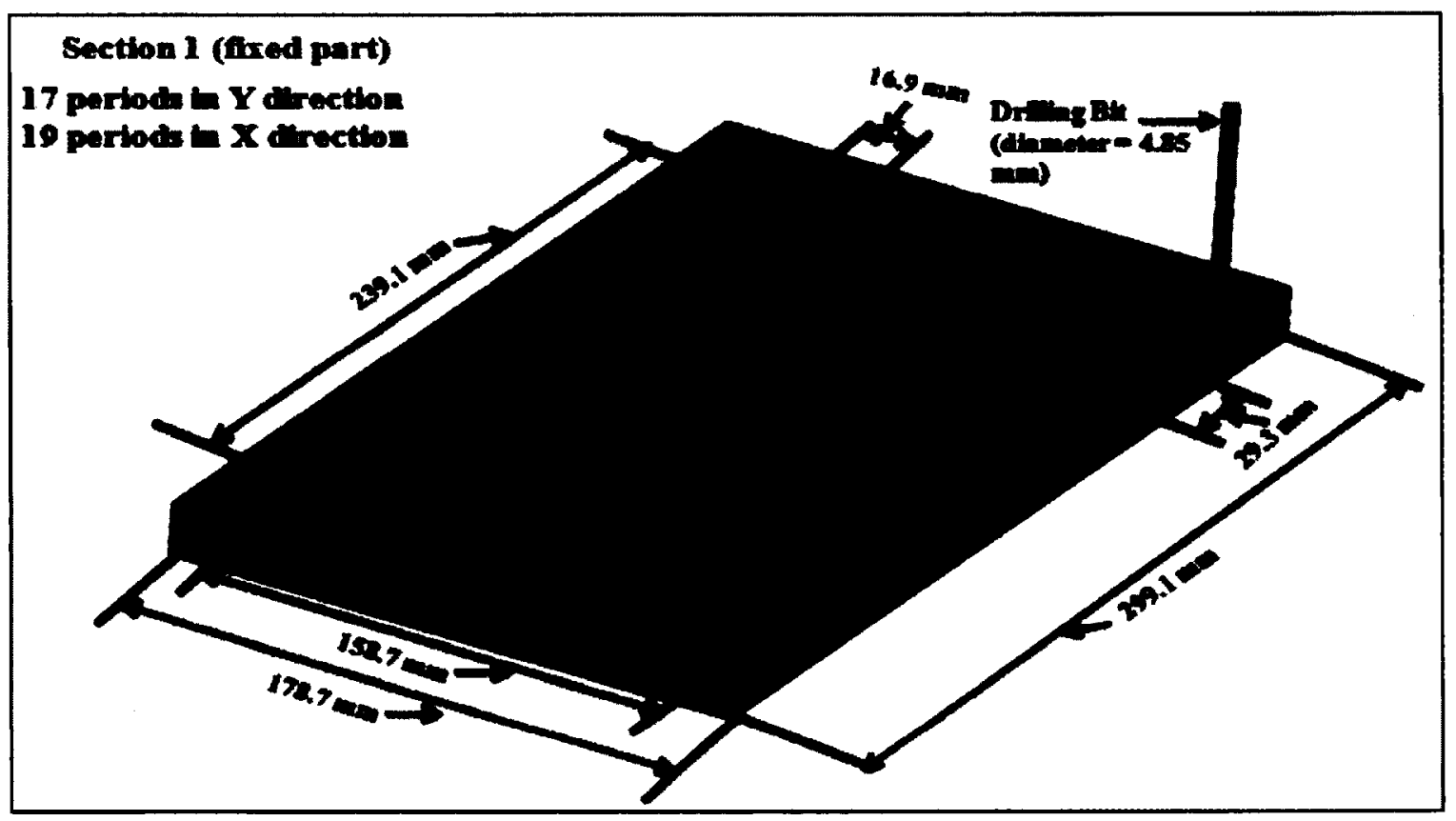

Figure 4.9: Section 1 - Fixed part with overall dimensions of $299.1 \mathrm{~mm} \mathrm{X} 178.7 \mathrm{~mm}$ consisting of 17 periods $(239.1 \mathrm{~mm})$ in the $\mathrm{Y}$ direction and 19 periods $(158.7 \mathrm{~mm})$ in the $\mathrm{X}$ direction to accommodate up to 152 alumina rods.

ii. Section 2-Plexi-glass strips:

This plexi-glass section contains a total of 32 strips having a width of $8.75 \mathrm{~mm}$ and a length of $299.1 \mathrm{~mm}$. There are two types of strips. Strip type 1, total 16 strips, having 8 holes of diameter, $\mathrm{d}=4.85 \mathrm{~mm}$ and the distance from center to center of each hole was $29.3 \mathrm{~mm}$, as shown in Figure 4.10. 


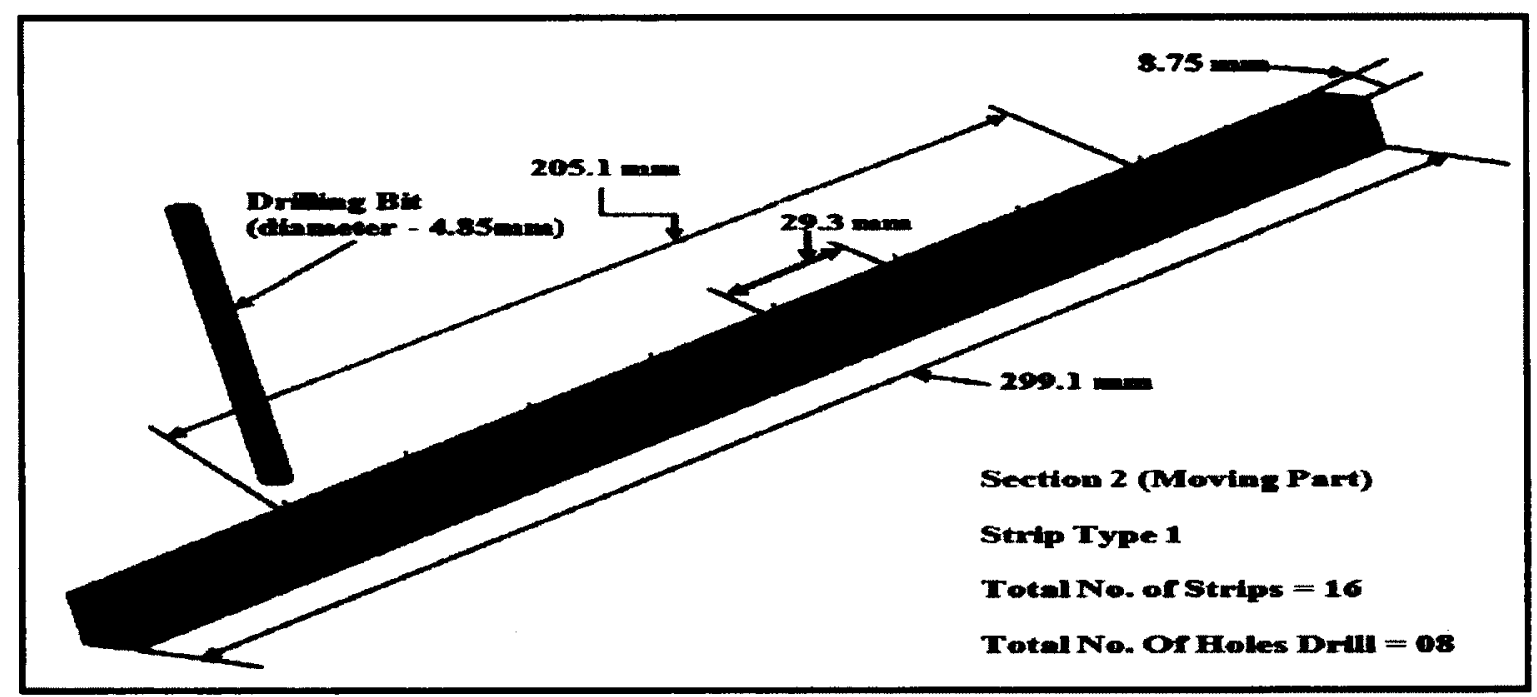

Figure 4.10: One of 16 strips, type 1, containing 8 holes of diameter, $d=4.85 \mathrm{~mm}$ and the distance from center to center of each hole was $29.3 \mathrm{~mm}$. The overall dimensions of the strips are $299.1 \mathrm{~mm} \mathrm{X} 8.75 \mathrm{~mm}$ to accommodate 128 alumina rods.

Strip type 2, total 16 strips, having 9 holes of diameter, $\mathrm{d}=4.85 \mathrm{~mm}$ and the distance from center to center of each hole was $29.3 \mathrm{~mm}$ as shown in Figure 4.11.

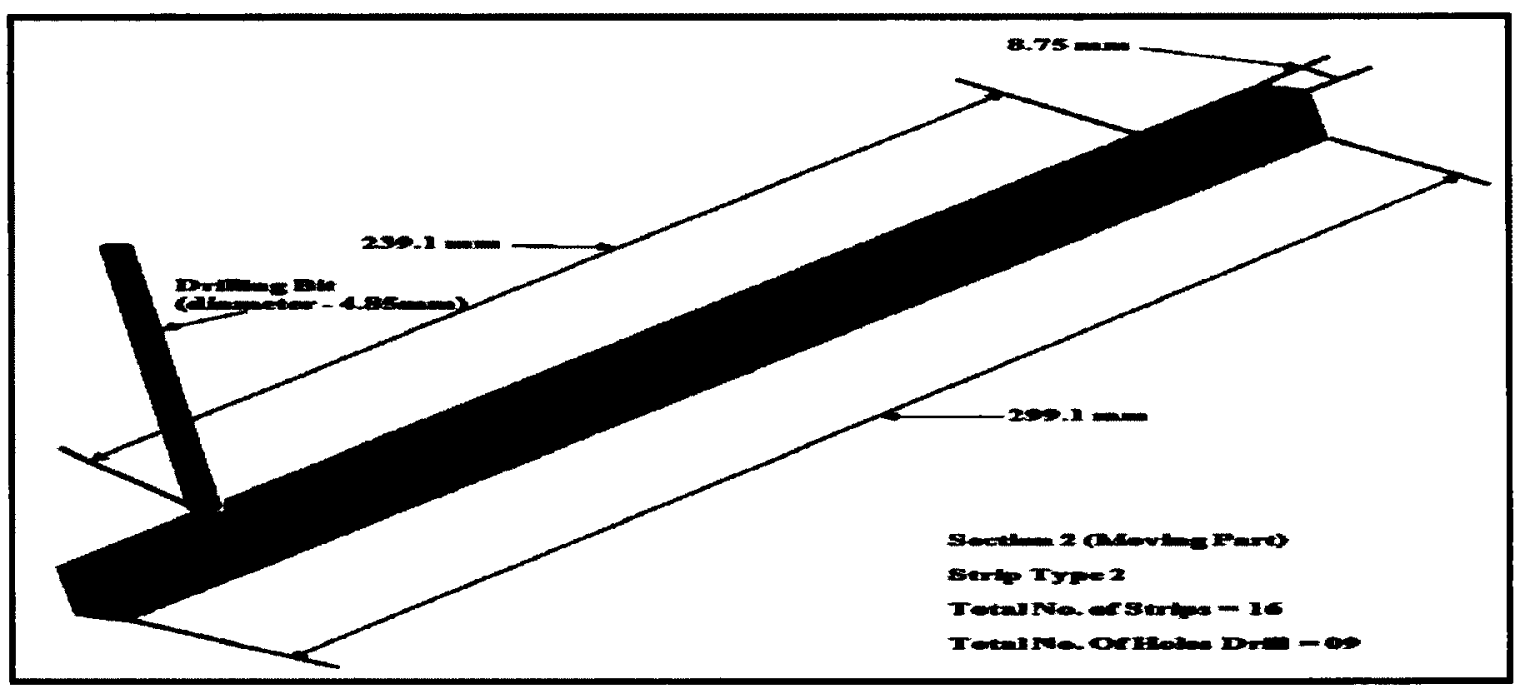

Figure 4.11: One of 16 strips, type 2 , containing 9 holes of diameter, $\mathrm{d}=4.85 \mathrm{~mm}$ and the distance from center to center of each hole was $29.3 \mathrm{~mm}$. The overall dimension of the strips is $299.1 \mathrm{~mm} \mathrm{X} 8.75 \mathrm{~mm}$ to accommodate 144 alumina rods. 
For fabricating a 2-D photonic crystal structure, these two types of strips (type 1 and type 2) were placed alternately. A total of 21 periods in the $\mathrm{X}$ direction and 17 periods in the $Y$ direction were used with overall dimensions of $183.75 \mathrm{~mm} \mathrm{X} 299.1 \mathrm{~mm}$ to accommodate 178 alumina rods ( 168 with the linear waveguide). The strip section of the plexi-glass template can be used to shift the rod array and provide the waveguide bends and $\mathrm{Y}$ branch.

\section{iii. Section 3 - Fixed Part:}

The overall dimensions of this section are $299.1 \mathrm{~mm}$ X $111.1 \mathrm{~mm}$. This section contains 17 periods $(239.1 \mathrm{~mm})$ in the $\mathrm{Y}$ direction and 11 periods $(91.1 \mathrm{~mm})$ in the $\mathrm{X}$ direction to accommodate up to 94 alumina rods as shown in Figure 4.12. To fabricate and perform experimental measurements, 17 periods in the $\mathrm{Y}$ direction and only 5 periods in the $\mathrm{X}$ direction were used to accommodate 43 alumina rods ( 40 with the linear waveguide). This section was moved together with section 2 (plexi-glass strips) and section 1 to form the microwave structure base template. Alumina rods with a rod radius, $r=4.85 \mathrm{~mm}$ were inserted into the holes of the template. In the next section, after arranging the alumina rods in a triangular pattern, microwave measurements were performed by placing a 2-D photonic crystal model between two horn antennas acting as transmitter and receiver for electromagnetic waves, which are connected to a network analyzer. 


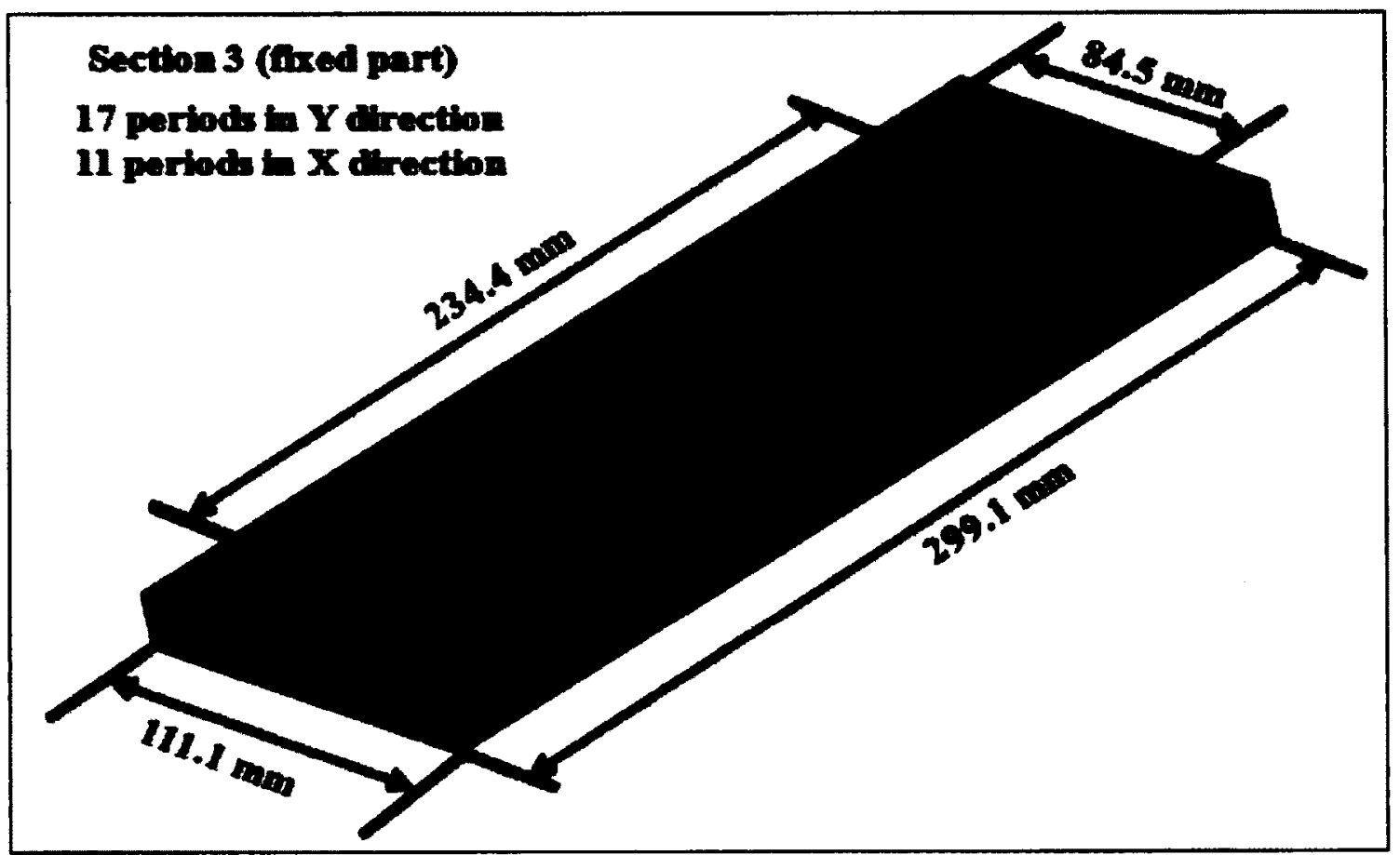

Figure 4.12: Section 3 - A fixed part with overall dimensions of $299.1 \mathrm{~mm}$ X $111.1 \mathrm{~mm}$ consisting of 17 periods $(239.1 \mathrm{~mm})$ in the $\mathrm{Y}$ direction and 11 periods $(91.1 \mathrm{~mm})$ in the $\mathrm{X}$ direction to accommodate up to 94 alumina rods.

4.7 Microwave Measurements for a 2-D Photonic Crystal (Without and With a Waveguide):

A 2-D photonic crystal that contained 17 periods in the $Y$ direction and 35 periods in the $\mathrm{X}$ direction accommodating 298 alumina rods was placed between the two horn antennas. The structure is shown in Figure 4.13. The transmission spectrum $S_{21}(\mathrm{~dB})$ versus frequency $(\mathrm{GHz})$ was obtained with and without the 2-D photonic crystal model as shown in Figure 4.14. 


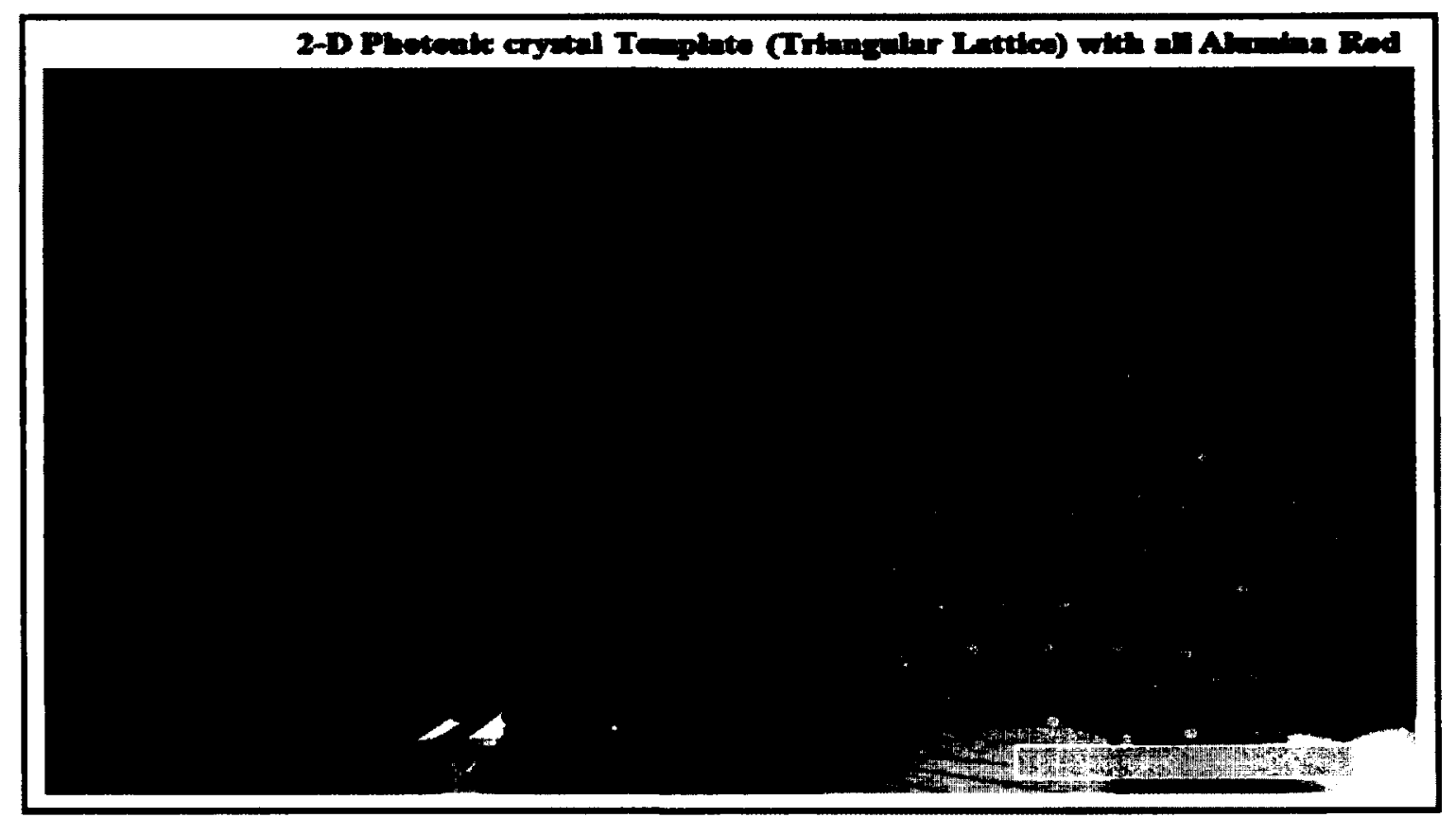

Figure 4.13: Picture of the alumina rods arranged in the triangular lattice using the base templates (lattice constant, $\mathrm{a}=16.9 \mathrm{~mm})$.

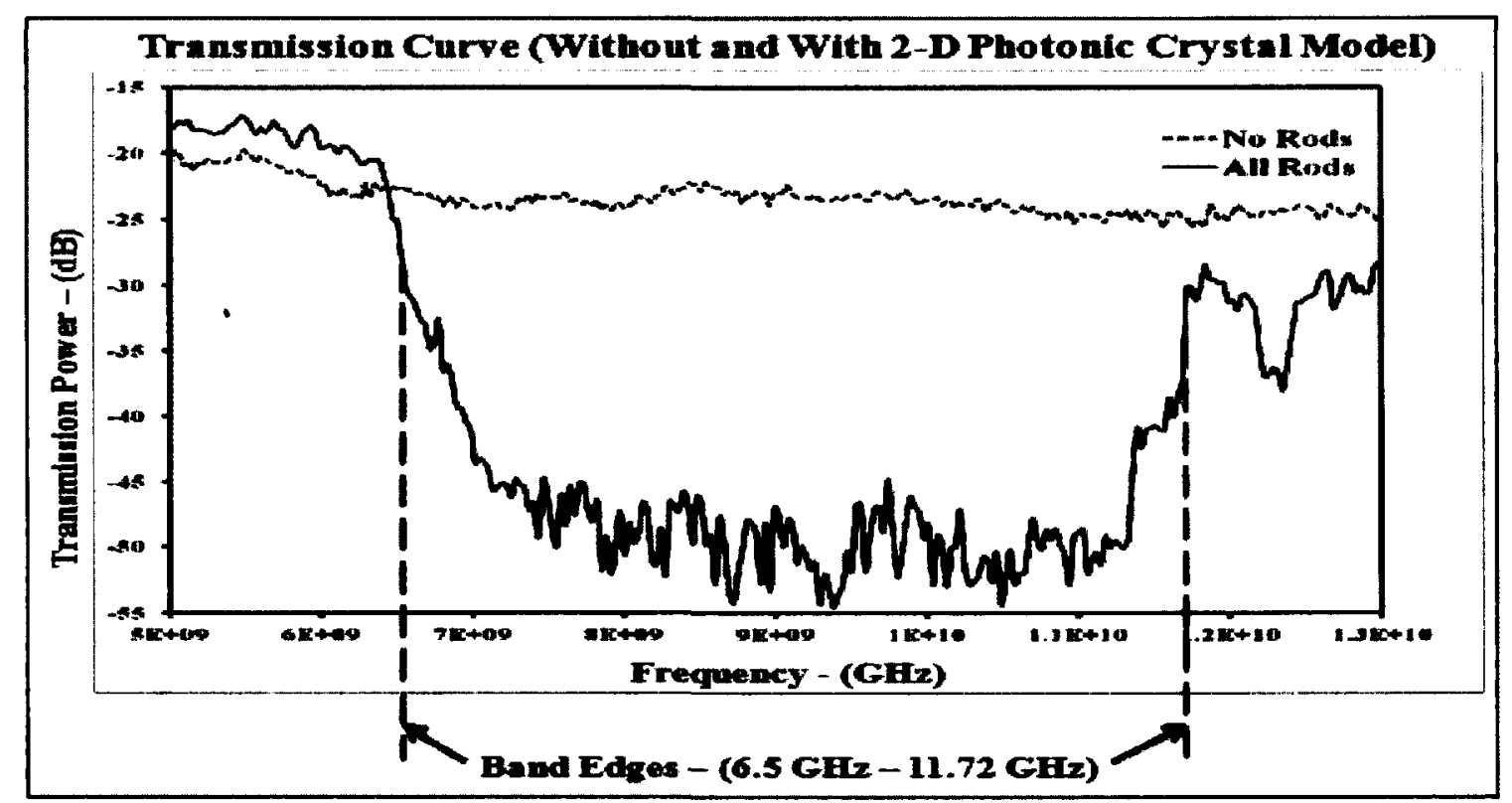

Figure 4.14: Transmission curves with and without a 2-D photonic crystal model present. The comparison of the curves shows the presence of the bandgap from $6.50 \mathrm{GHz}$ to $11.72 \mathrm{GHz}$ when the structure is in place. 
It was found that there is a difference of around $4 \mathrm{~dB}$ between two transmission curves at the low frequency side. This rise is due to the photonic crystal providing some channelling of the electromagnetic waves. The high frequency side shows a lower transmission as scattering losses from the rods dominate over the channelling. The transmission curve with the 2-D photonic crystal structure shows a large photonic bandgap from $6.5 \mathrm{GHz}$ to $11.72 \mathrm{GHz}$. This photonic bandgap is in excellent agreement with those predicted using the plane wave and FDTD methods as discussed in Chapter 3 (refer to Figure 3.4, Figure 3.6 and Figure 3.9).

A line waveguide with width, $\mathrm{W}=24.45 \mathrm{~mm}$ was introduced along the $\mathrm{X}$ direction by removing the ninth row of rods (center row) as shown in Figure 4.15. This provides a channel for bandgap frequency modes to propagate from source to detector through the crystal. The transmitted signal $S_{21}$ is shown in Figure 4.16 along with the full crystal signal showing the bandgap and background reference.

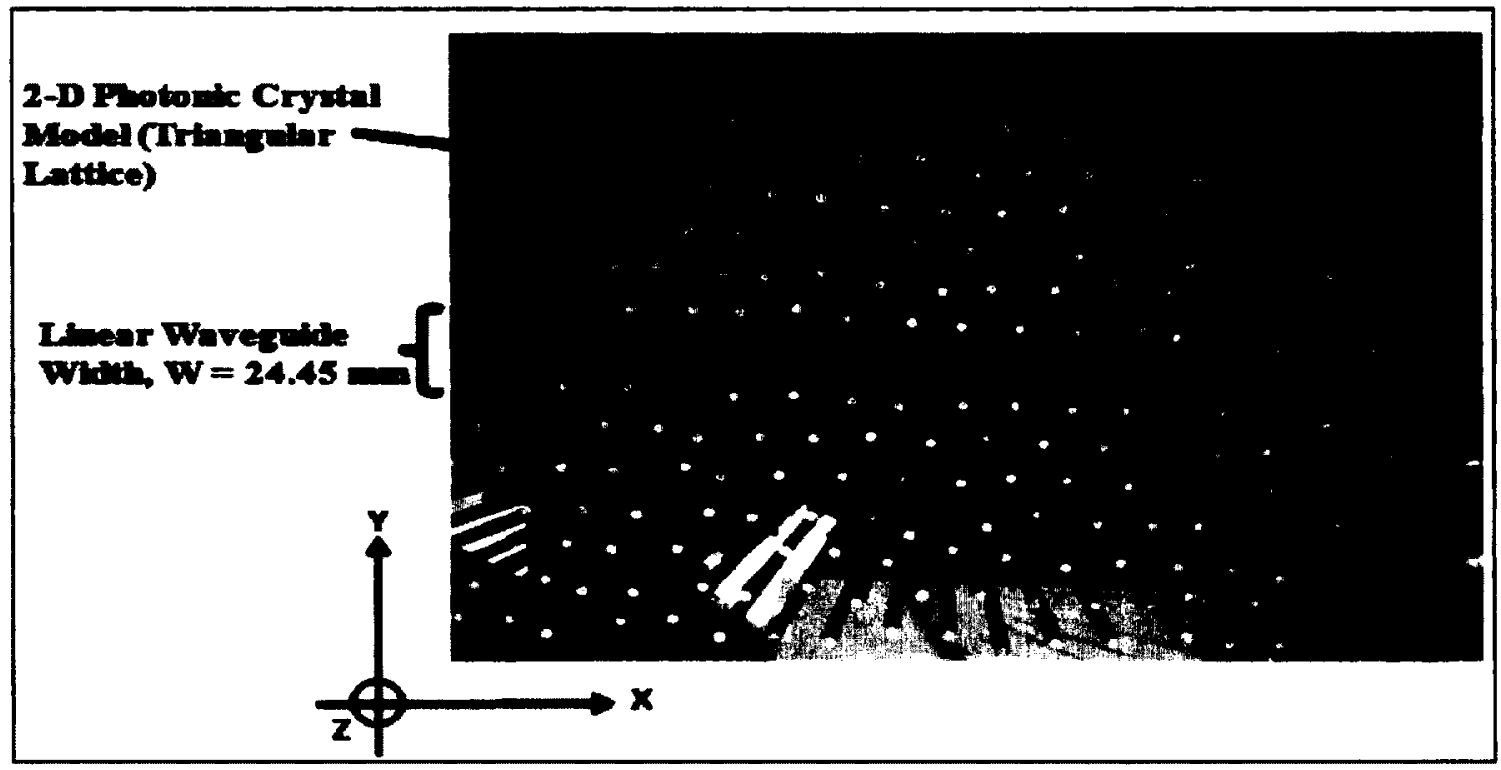

Figure 4.15: Picture of a 2-D linear waveguide within the triangular lattice. The waveguide has width, $\mathrm{W}=$ $24.45 \mathrm{~mm}$. 


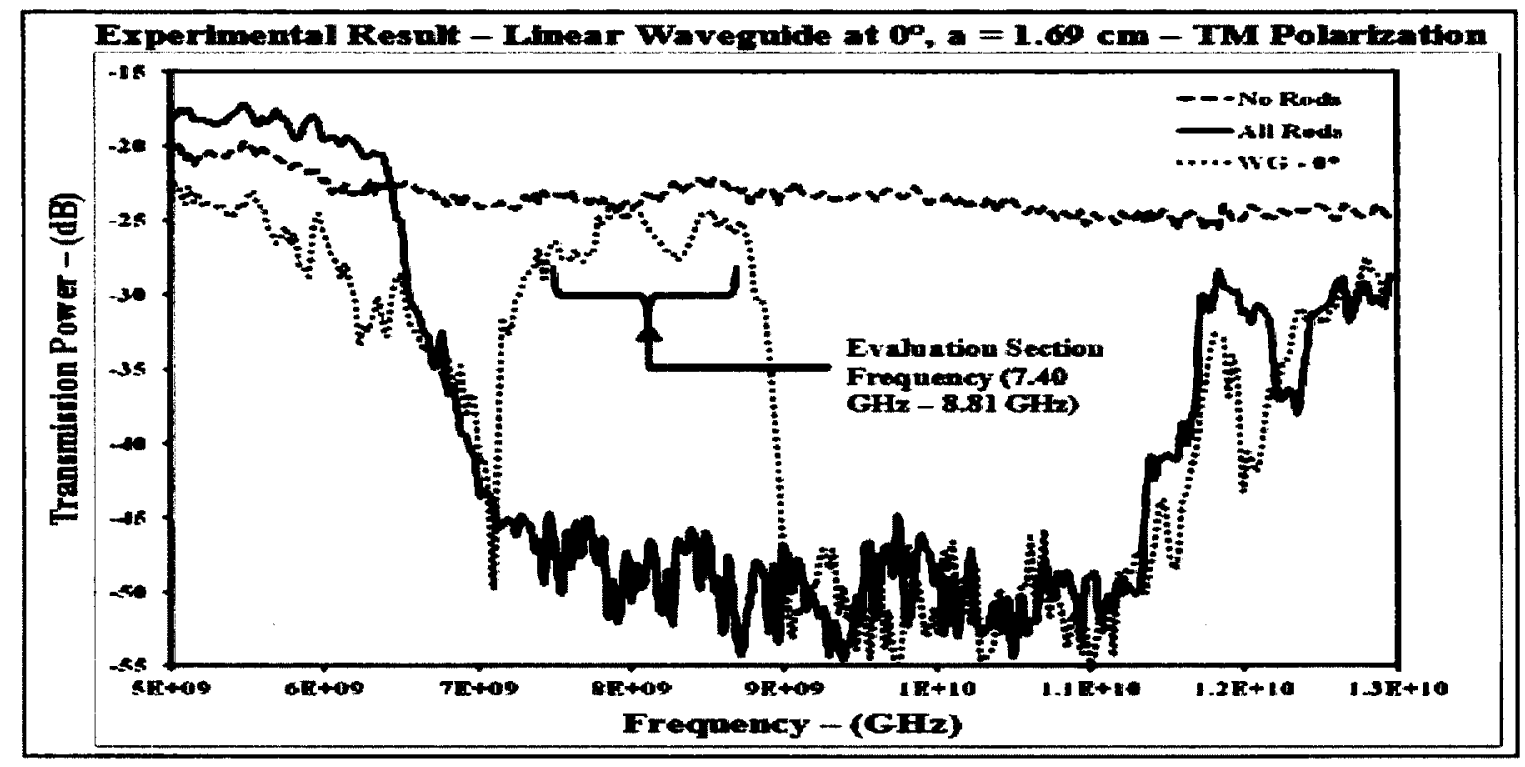

Figure 4.16: Transmission spectrum obtained in the microwave regime when the triangular array is fully populated (bandgap) and when one row of rods is removed (waveguide). The reference trace is obtained by taking a spectrum with the rod array fully removed.

The band of frequencies propagated within the band gap region are identified as existing between $7.40 \mathrm{GHz}$ to $8.81 \mathrm{GHz}$, and given the scalability that exists between microwave and optics, corresponds well with the predicted transmission band computed using the plane wave and FDTD techniques. Figure 4.16 indicates that a suitable test environment has been established in which an optical photonic crystal can be experimentally evaluated at microwave frequencies.

In the next chapter, a brief description of the design and fabrication of a 2-D photonic crystal double bend waveguide, both in the optical and microwave regimes at different waveguide bend angles, $\theta$ varying from $0^{\circ}$ to $30^{\circ}$, will be discussed. Finally, the design of the $\mathrm{Y}$ branch splitter with an optimal bend angle in the microwave regime will be presented with results. 


\section{Double Bend Waveguide Measurements and Y Branch Structure}

In the previous chapters ( 3 and 4 ), a brief description of simulation techniques in the optical regime and experimental setup to perform various measurements in the microwave regime were discussed. The results of both the plane wave and FDTD methods show good agreement for a photonic bandgap. The photonic bandgap obtained for a 2-D photonic crystal, both in the optical and microwave regime, were found to be in good agreement after applying the scalability property. A waveguide was created within a 2-D photonic crystal of a channel width, $\mathrm{W}=24.45 \mathrm{~mm}$ as referenced in Figure 4.15 that has a guided band $(7.40 \mathrm{GHz}$ to $8.81 \mathrm{GHz}$ ) lying inside the TM bandgap ( $6.5 \mathrm{GHz}$ to $11.72 \mathrm{GHz}$ ). The waveguide propagation between simulations and experiments also agrees well through scalability.

This chapter explains the effect of introducing a double bend in the waveguide and is examined both in the optical and microwave regimes. The bending process is extended to a Y branch waveguide splitter.

\subsection{Double Bend Waveguide Formation}

The basic waveguide configuration of the previous chapter has been modified to include bends. The photonic crystal waveguide structure with bends present is shown in Figure 5.1. The black region represents the high dielectric alumina rods arranged in a triangular array and the white background is air. A total of 15 periods were used in the $\mathrm{Y}$ direction 
and 39 periods in the $\mathrm{X}$ direction ( 9 periods (input), 21 periods (incremental sheer), 9 periods (output)).

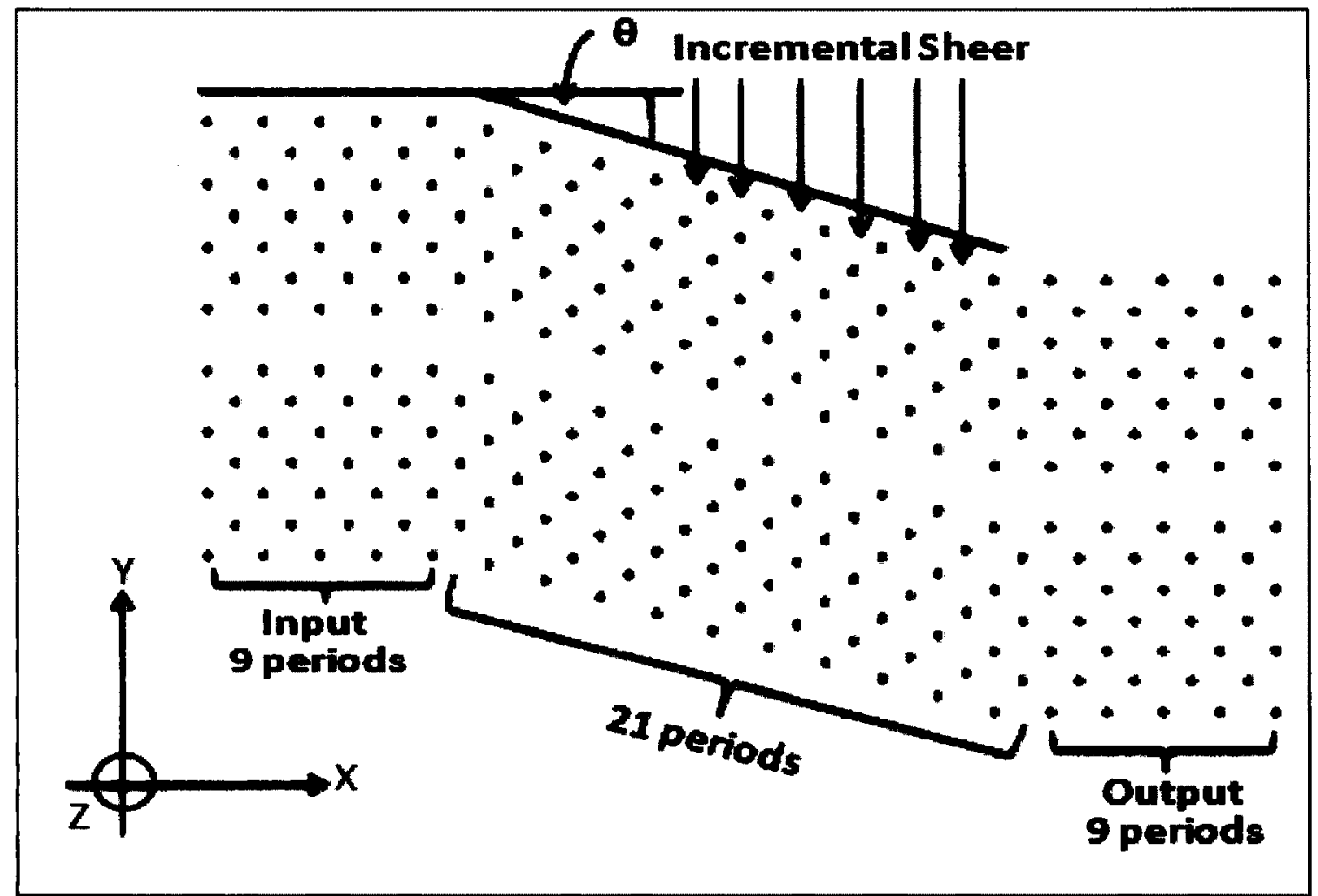

Figure 5.1: The waveguide configuration with double bend present. Lattice is triangular with high dielectric rods shown in black and air in background in white. The bend in the waveguides is parameterized based on the inclination angle between input and shifted photonic crystal segments. 
The double bends in the waveguide are produced by incrementally shifting the rods down over the middle segments of the photonic crystal. In this way, the input and output waveguides remain parallel, but laterally offset by an amount related to the number of periods in the shift region and level of shift. A suitable parameter to monitor the level of the bend in the waveguide is to quote the bend angle between the input waveguide and the shifted waveguide segment $(\theta)$. This bend technique was implemented in the microwave regime using the plexi-glass template structure. In the optical domain, the theoretical analysis was facilitated by specifying the location of each rod.

\subsubsection{FDTD Results}

Transmission spectrums were obtained after doing FDTD analysis of a 2-D photonic crystal structure (TM polarization) using the FDTD simulator (for more details refer to Figures (3.9), (3.10), (3.11), (3.12)).

After analyzing Figures (3.10) and (3.12), a conclusion was drawn that there is an existence of photonic bandgap at wavelength, $\lambda=1.28 \mu \mathrm{m}$ to $2.02 \mu \mathrm{m}$, and there is a guided band (a certain range of wavelength $(1.35 \mu \mathrm{m}$ to $1.85 \mu \mathrm{m})$ that is guided through a waveguide channel. This range of wavelength $(1.35 \mu \mathrm{m}$ to $1.85 \mu \mathrm{m})$ coincides within the range of wavelengths $(1.35 \mu \mathrm{m}$ to $1.74 \mu \mathrm{m})$ obtained with the help of a supercell technique and the plane wave method. After analyzing both simulation results (FDTD and supercell), a single wavelength, $\lambda=1.45 \mu \mathrm{m}$ was chosen for further FDTD simulation because it showed the maximum output power. FDTD simulations were performed on 2-D linear waveguides for different waveguide bend angles, $(\theta)$ varying 
from $1^{\circ}$ to $15^{\circ}$ in $1^{\circ}$ increments and from $16^{\circ}$ to $30^{\circ}$ in $5^{\circ}$ increments at $\lambda=1.45 \mu \mathrm{m}$. The transmission spectrums were obtained and a plot was created showing transmission loss versus waveguide bend angle, $(\theta)$ as shown in Figure 5.2. The transmission loss (dB) was calculated by taking the ratio between the lowest transmission signal obtained for a linear waveguide at $0^{\circ}$ to the transmission signal obtained for the same structure at different waveguide bend angles, $(\theta)$ varying from $1^{\circ}$ to $30^{\circ}$ in $1^{\circ}$ increments at $\lambda=1.45$ $\mu \mathrm{m}$.

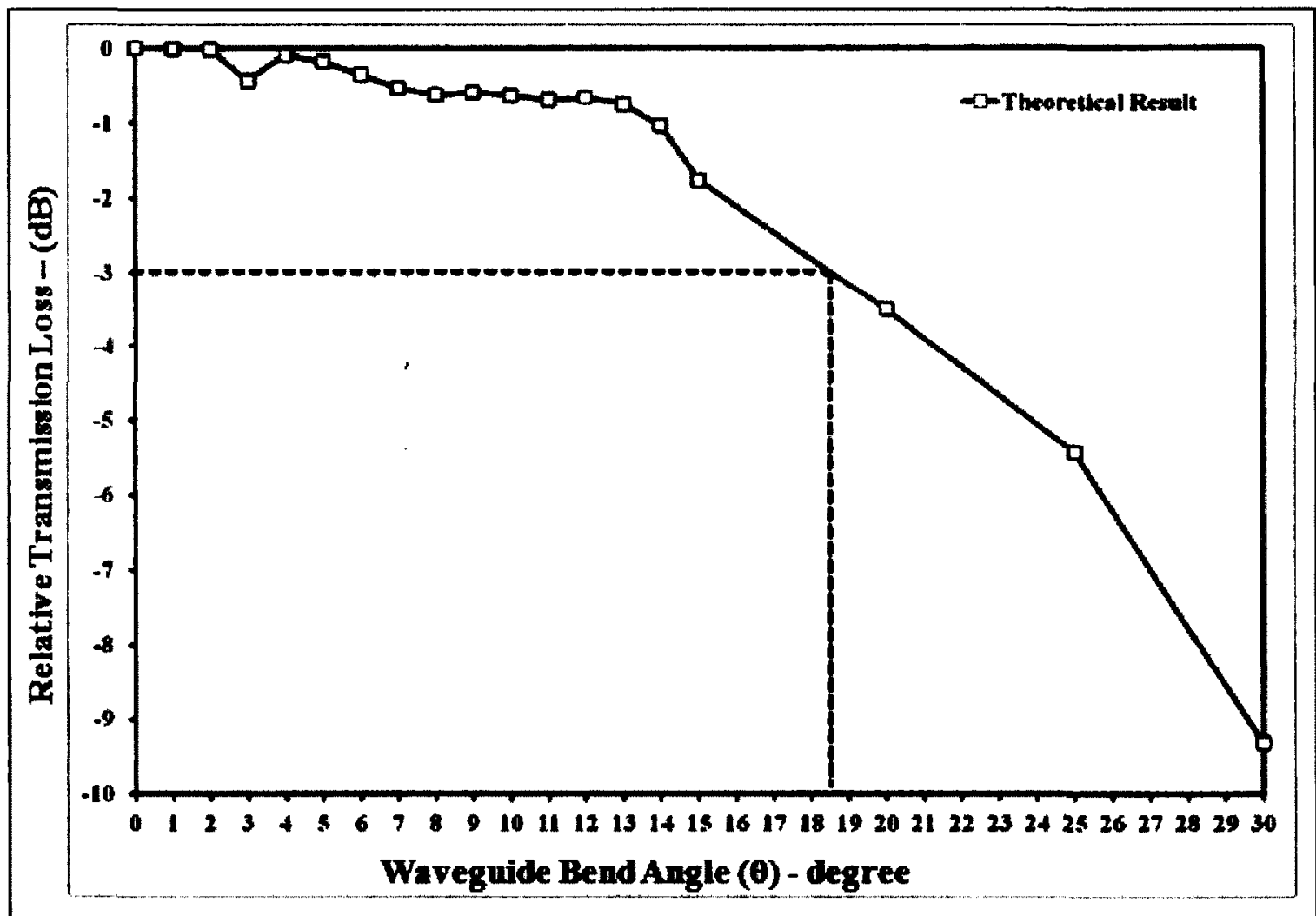

Figure 5.2: Plot of transmission loss (dB) versus waveguide bend angle, $(\theta)$ obtained after analyzing transmission spectrums at wavelength, $\lambda=1.45 \mu \mathrm{m}$, with different waveguide bend angles, $\left(\theta=0^{\circ}\right.$ to $\left.30^{\circ}\right)$. 
The trace indicates that for some angles the transmission power increases with the increasing bend angle. This occurs due to the inclined waveguide forming a cavity between bends and producing a resonator. Below a waveguide bend of $18^{\circ}$ the additional transmission loss is less than $3 \mathrm{~dB}$.

\subsubsection{Microwave Experimental Results:}

A calibration procedure (refer to Subsection (4.1.1)) of network analyzer and complete experimental set up (refer to Section 4.3 and 4.7) were discussed in Chapter 4. By following the experimental procedure, the $S_{21}$ parameter was obtained from the network analyzer with the help of the GPIB connected to a computer. The whole procedure to get the reference transmission curve (without placing an array between the transmitter and receiver) and transmission curve with the 2-D photonic crystal model were obtained as per the directions already discussed in Chapter 4 under Section 4.7. Full spectrums were obtained for each waveguide bend angle and the measurements were repeated five times (full dismount and remounting of experimental setup) in order to perform a statistical analysis on the waveguide propagation properties.

Figure 5.3 shows a transmission spectrum for a linear waveguide at bend angle, $\theta$ $=0^{\circ}$ along with the reference and full crystal spectrums. These traces are produced from Figure 4.16. The transmission drop throughout the bandgap is $20 \mathrm{~dB}$ with a bandwidth of $3.40 \mathrm{GHz}$. There are two transmission windows where the output is at the maximum. A first window lies at a frequency range of $7.88 \mathrm{GHz}$ to $8.03 \mathrm{GHz}$ and the second window lies in frequency ranges from $8.45 \mathrm{GHz}$ to $8.81 \mathrm{GHz}$. 


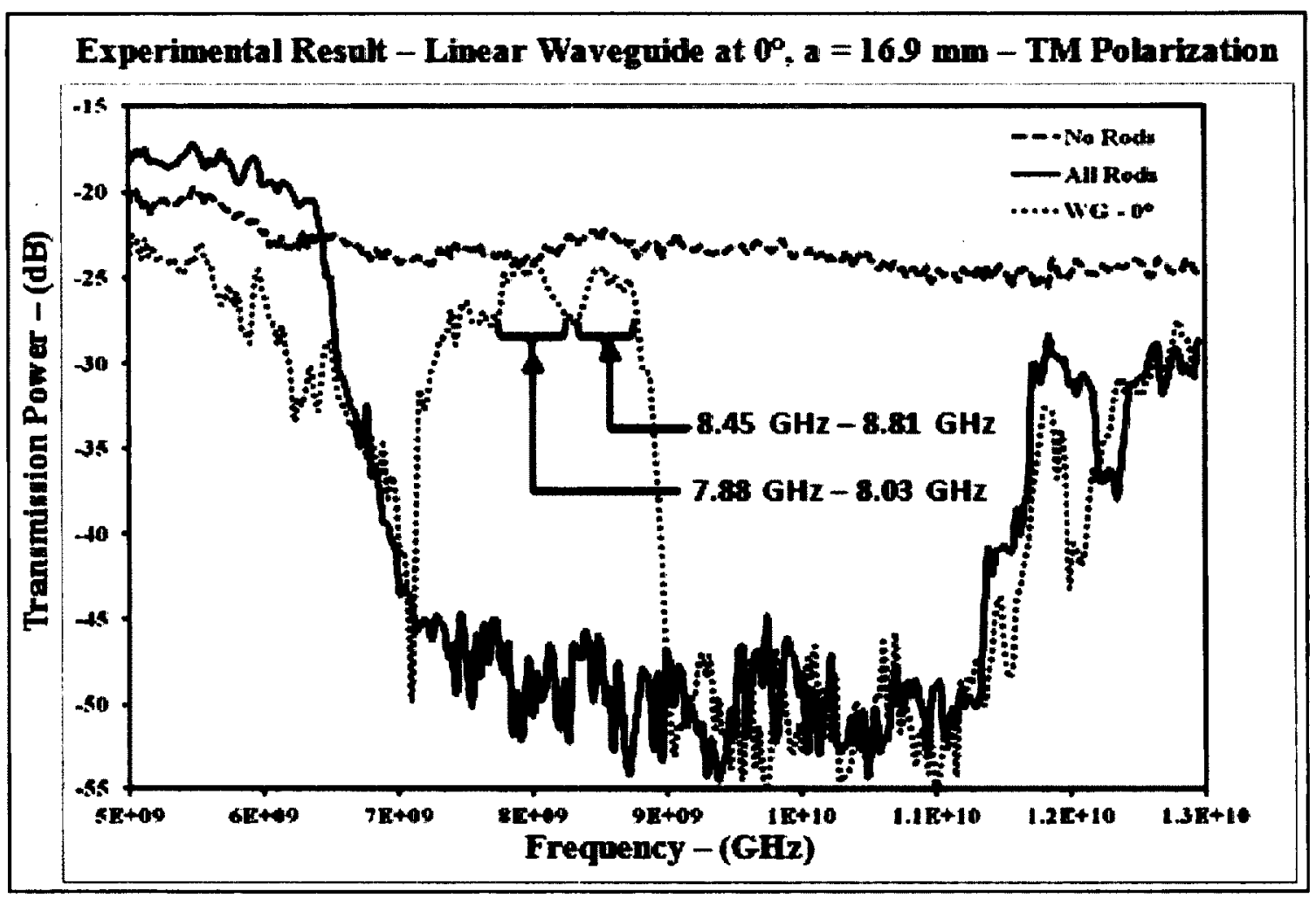

Figure 5.3: Transmission spectrum for a linear waveguide with lattice constant, $a=16.9 \mathrm{~mm}$ at bend angle, $\theta=0^{\circ}$ showing the photonic bandgap $(\mathrm{f}=6.5 \mathrm{GHz}$ to $11.72 \mathrm{GHz}$ ) with a range of frequencies $(\mathrm{f}=7.40$ $\mathrm{GHz}$ to $8.81 \mathrm{GHz}$ ) that is getting through the waveguide channel.

A dielectric shift technique was introduced to form a double bend waveguide with the waveguide bend angle, $\theta$ varying from $1^{\circ}$ to $30^{\circ}$ as referenced in Figure 5.1. The input and output waveguide segments of the array were maintained intact and the central region (incremental shear) had the plexi-glass strips incrementally shifted, such that angular increments of $1^{\circ}$ were introduced. Microwave measurements were made and 
transmission curves (transmission power versus frequency) were obtained for different waveguide bend angles, $\theta\left(1^{\circ}\right.$ to $\left.30^{\circ}\right)$ with $\mathrm{TM}$ polarization.

Figure 5.4 shows a microwave experimental view for a 2-D photonic crystal model consisting of a double bend waveguide at $\theta=10^{\circ}$ with a triangular lattice having a lattice constant, $\mathrm{a}=16.9 \mathrm{~mm}$.

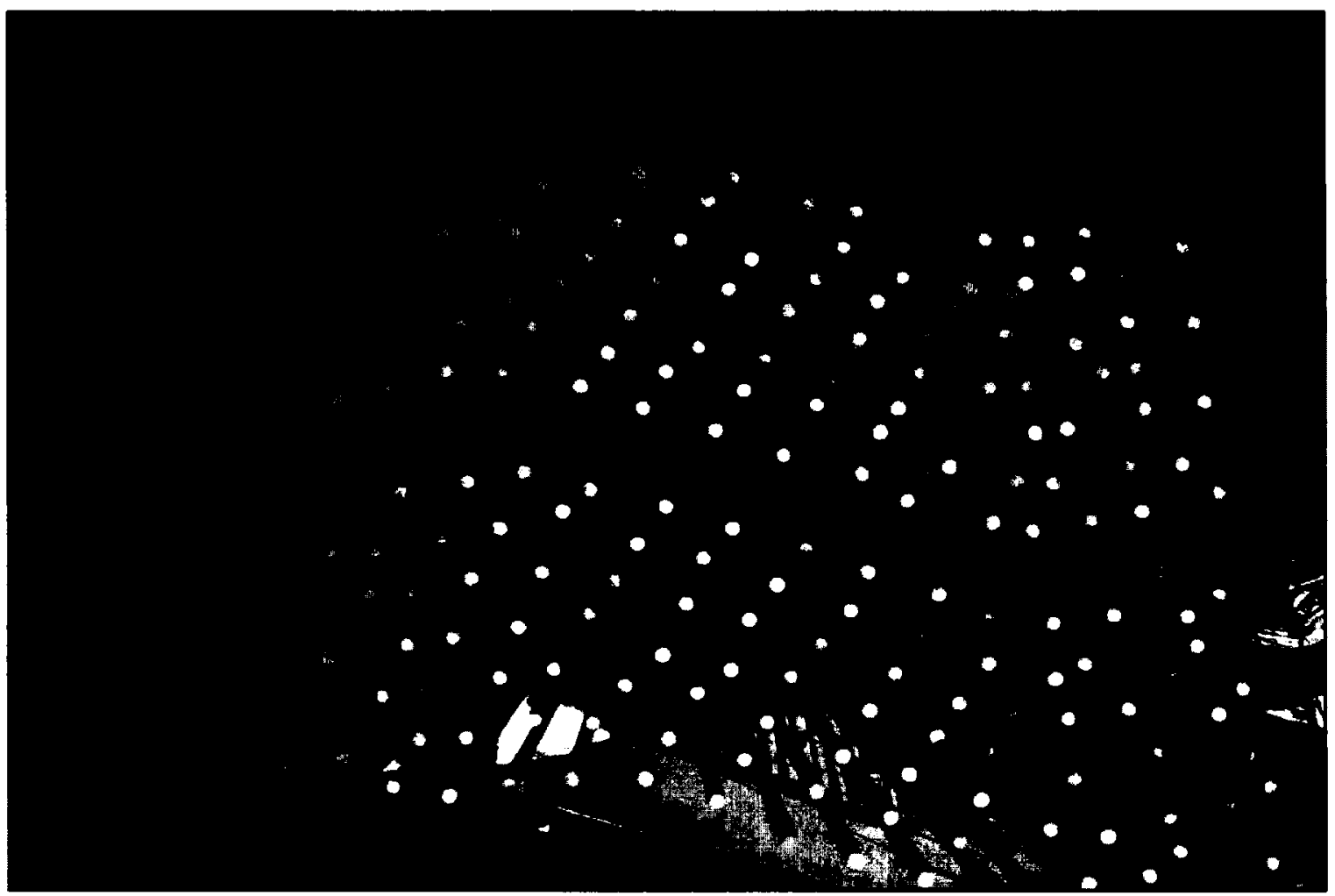

Figure 5.4: Picture of the 2-D photonic crystal model showing double bends at $\theta=10^{\circ}$ with an incremental shear section between the input and output sections.

Figures 5.5 and 5.6 show a full transmission spectra graphical layout where the waveguide offset angle, $\theta$ varied from $1^{\circ}$ to $30^{\circ}$ with the frequency range from $5 \mathrm{GHz}$ to 
$13 \mathrm{GHz}$, and observations were made after analyzing these graphical images. Full page view of these figures can be seen in Appendix C.

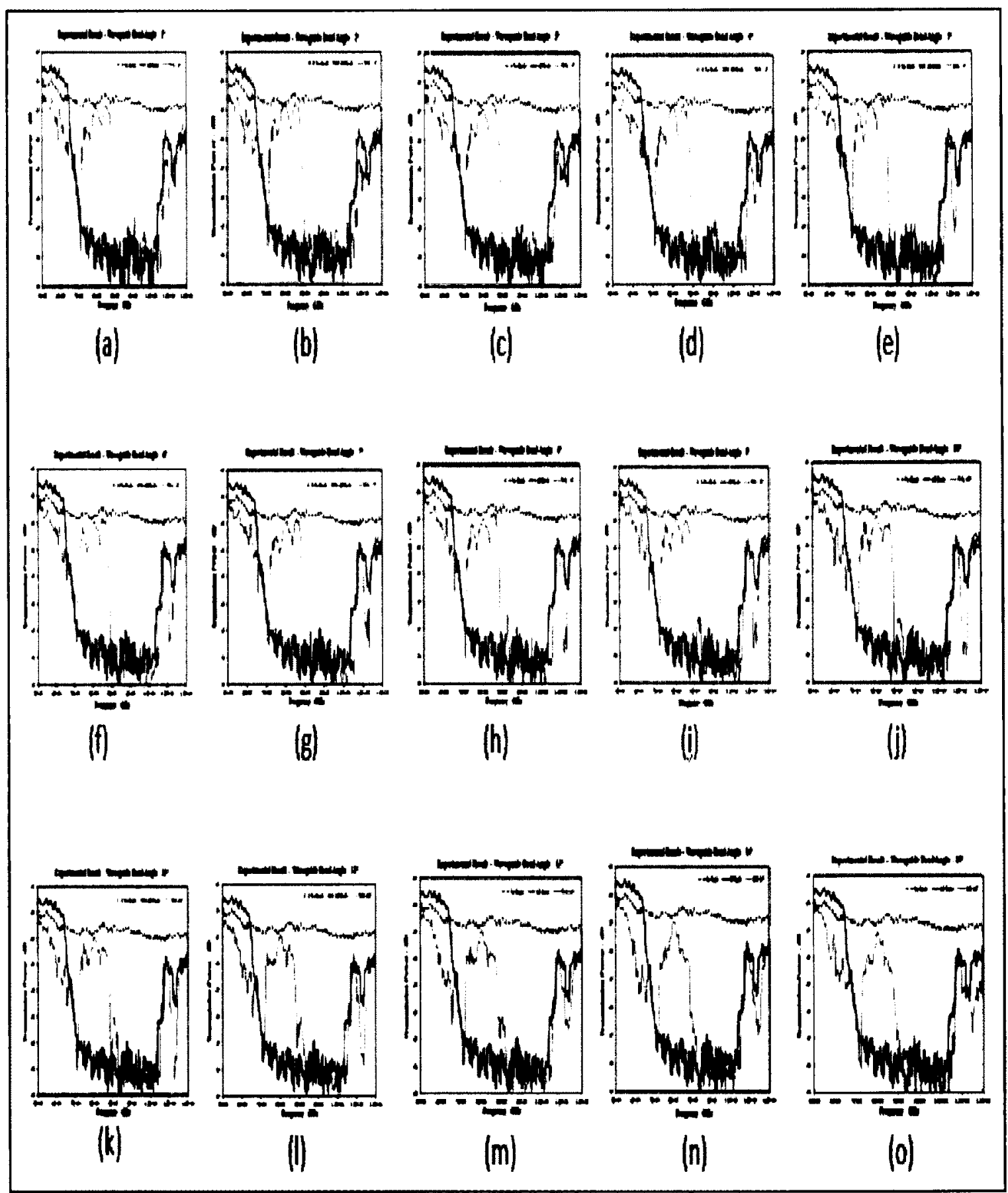

Figure 5.5: (a-o) shows transmission curves (transmission power $(\mathrm{dB})$ versus frequency $(\mathrm{GHz})$ for different waveguide offset angles $\left(1^{\circ}\right.$ to $\left.15^{\circ}\right)$. Refer to Appendix $\mathrm{C}$ for full page view. 


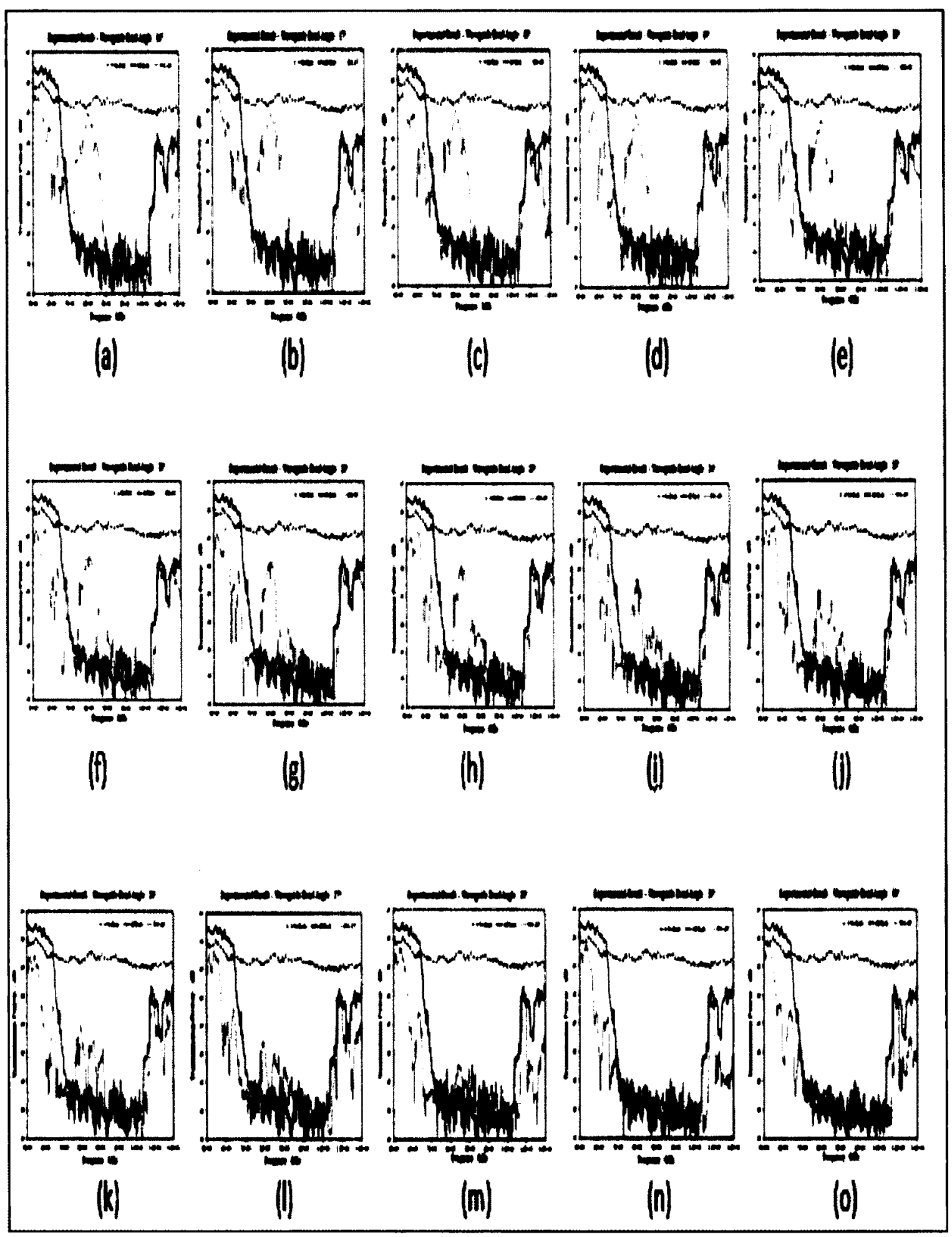

Figure 5.6: (a-o) shows transmission curves (transmission power $(\mathrm{dB})$ versus frequency $(\mathrm{GHz})$ for different waveguide offset angles $\left(16^{\circ}\right.$ to $\left.30^{\circ}\right)$. Refer to Appendix $C$ for full page view. 
The raw data containing the S-parameters were obtained from the network analyzer and stored on a personal computer with the help of GPIB. From these Sparameters, only $S_{21}$ was evaluated and systematically organized to get the transmission spectrums. Note: To record the transmission as a function of the direction of propagation, the middle section (incremental shear section) of a 2-D model was shifted down by increments of $1^{\circ}$ with respect to the normal direction, while keeping the position of the transmitter antennas unchanged.

Figure 5.7 shows a 3-D line plot to summarize Figures 5.5 and 5.6. This graph shows the transmission properties of the linear waveguide at different waveguide bend angles, $\theta$ for frequencies ranging from $7 \mathrm{GHz}$ to $9.26 \mathrm{GHz}$. 


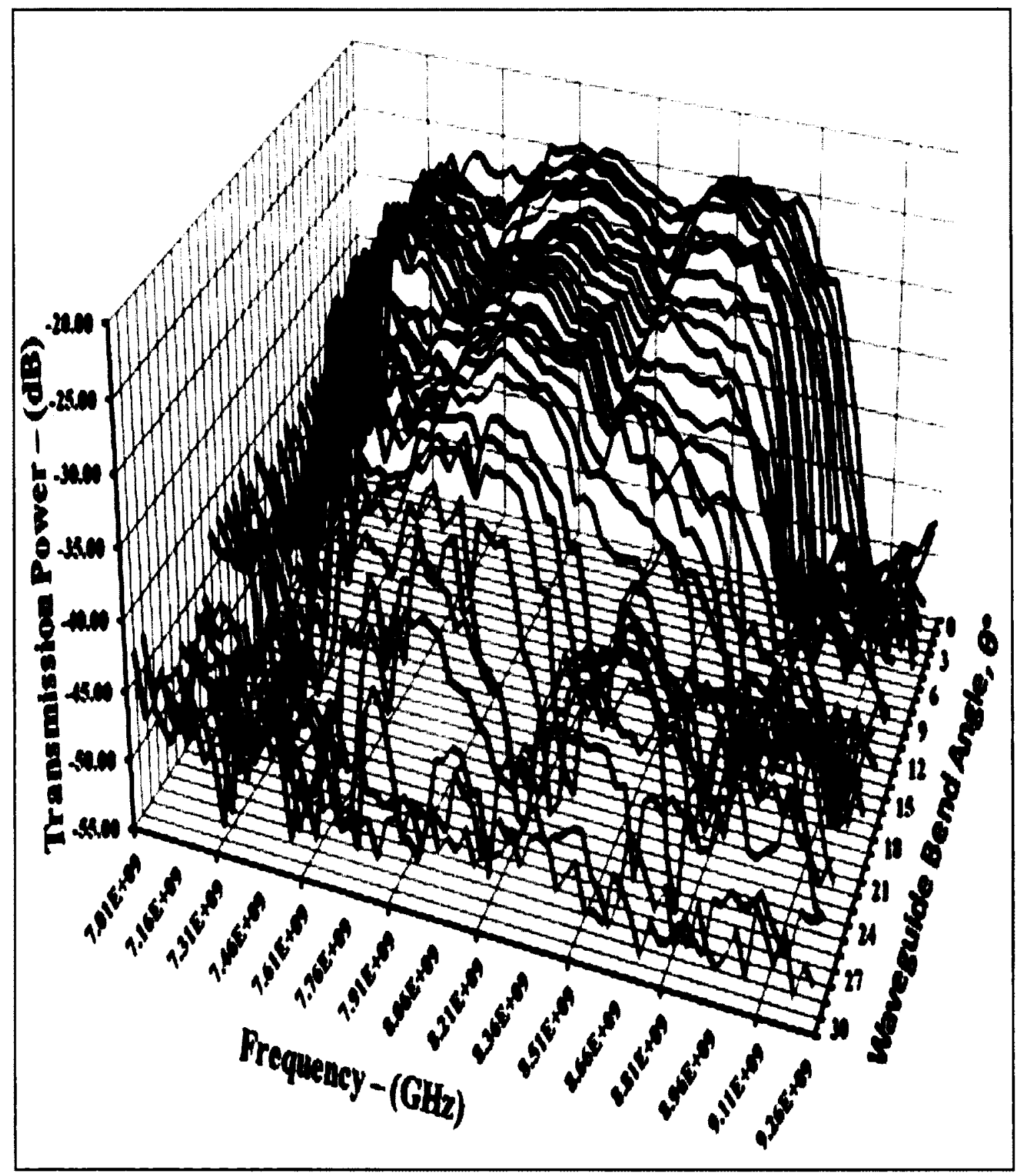

Figure 5.7: 3-D line plot showing the transmission power for a range of frequencies $(7 \mathrm{GHz}$ to $9.26 \mathrm{GHz})$ at different waveguide bend angles, $\theta$ varying from $0^{\circ}$ to $30^{\circ}$. 
As previously indicated in Figure 5.3, two high transmission windows were selected for further evaluation. Figure 5.8 (a), (b) and Figure 5.9 (a), (b) were constructed to see the transmission behavior at selected groups of frequencies at different waveguide bend angles, $\theta$.

The relative transmission loss was calculated by taking the ratio of transmission signal (point) obtained when the 2-D template (fully populated with alumina rods) was placed between the receiver and transmitter at a particular frequency to the transmission signal (point) obtained when a linear waveguide is formed within the template and a waveguide bend is introduced at a particular waveguide bend angle, $\theta$ at a particular frequency. After obtaining the relative transmission loss for five different microwave measurements, the average relative transmission loss was calculated by taking the average of all readings for a particular frequency at a particular waveguide bend angle, $\theta$. 


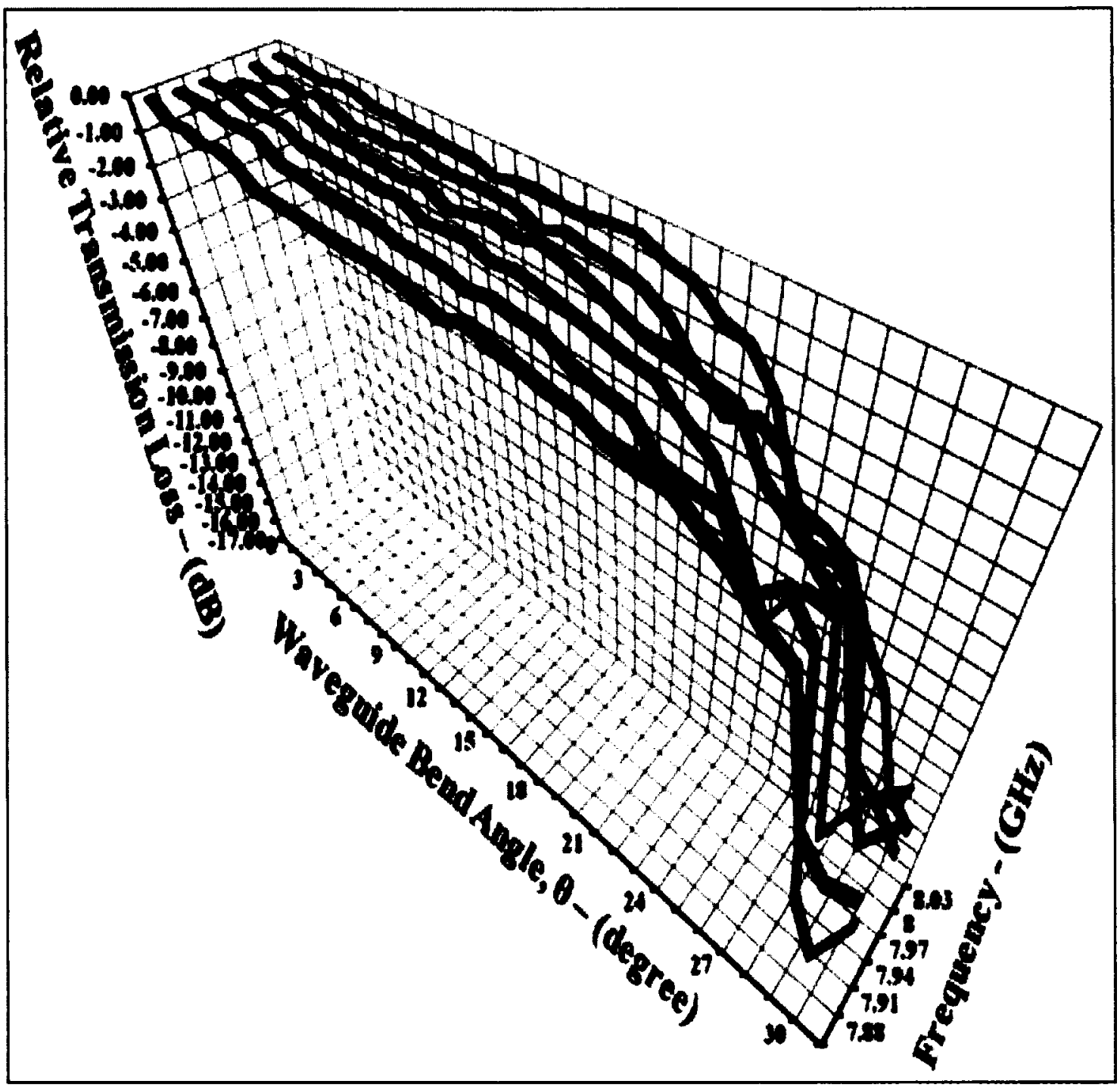

Figure 5.8 (a): 3-D line plot for a frequency range of $7.88 \mathrm{GHz}$ to $8.03 \mathrm{GHz}$ showing a relative transmission loss at different waveguide bend angles, $\theta$, varying from $0^{\circ}$ to $30^{\circ}$. 


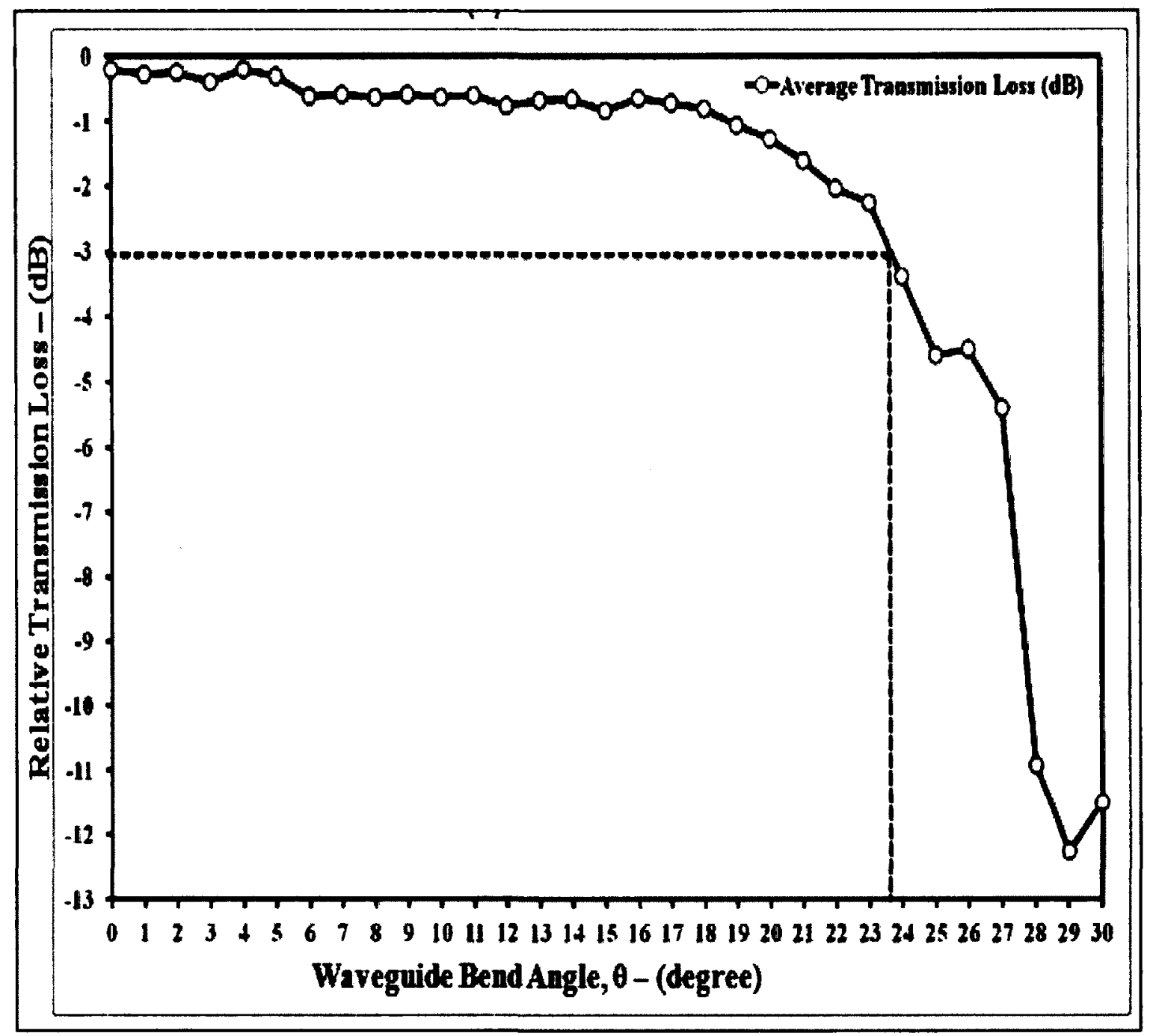

Figure 5.8 (b): Plot showing the average transmission loss for a frequency interval ( $7.88 \mathrm{GHz}$ to $8.03 \mathrm{GHz}$ ) at different waveguide bend angles, $\theta$, varying from $0^{\circ}$ to $30^{\circ}$.

Figure 5.8 (b) shows the average transmission loss of less than $3 \mathrm{~dB}$ up to the waveguide bend angle, $\theta=23^{\circ}$ for a frequency range between $7.88 \mathrm{GHz}$ to $8.03 \mathrm{GHz}$. 


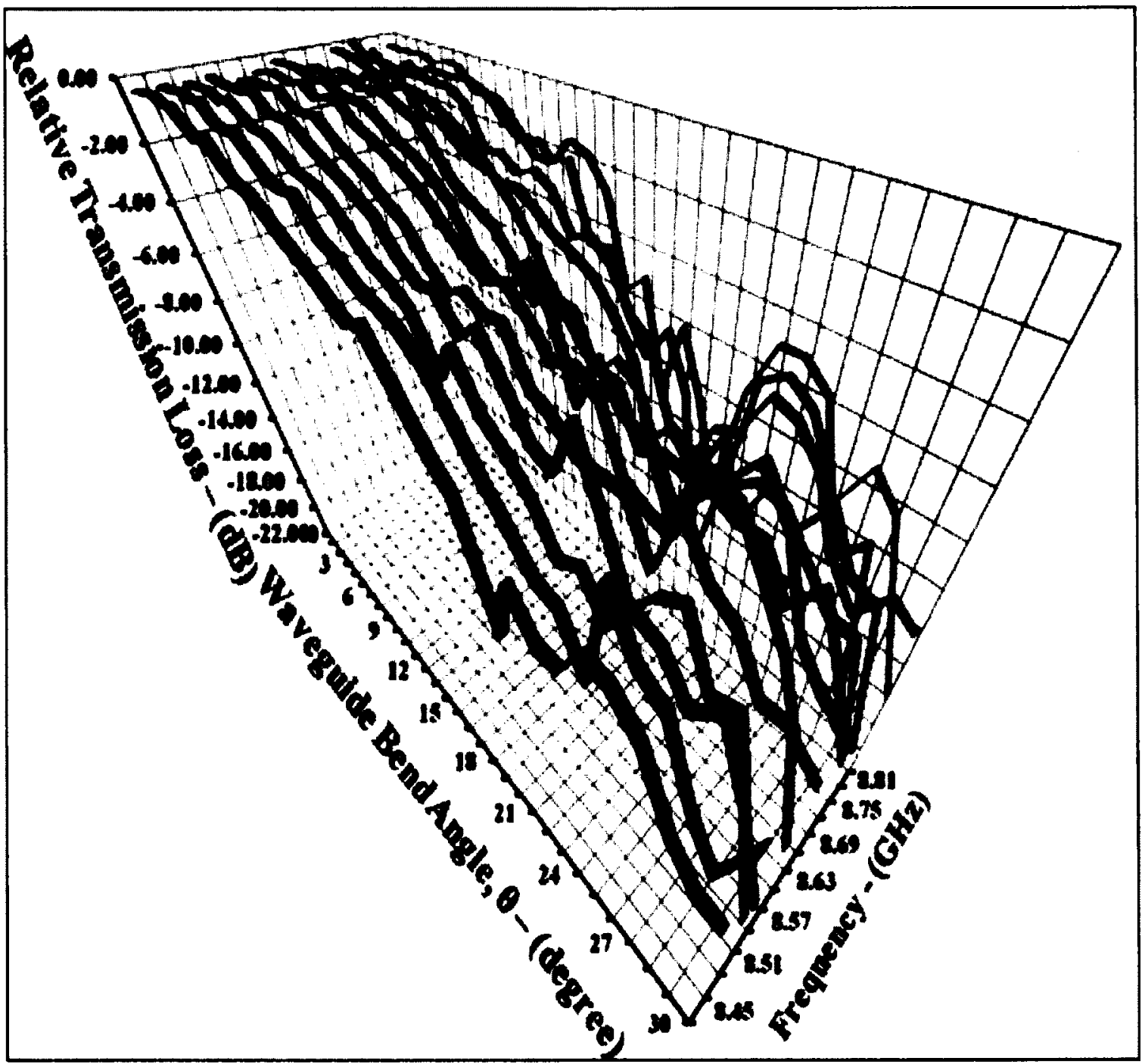

Figure 5.9 (a): 3-D line plot for a frequency range of $8.45 \mathrm{GHz}$ to $8.81 \mathrm{GHz}$ showing a relative transmission loss at different waveguide bend angles, $\theta$, varying from $0^{\circ}$ to $30^{\circ}$. 


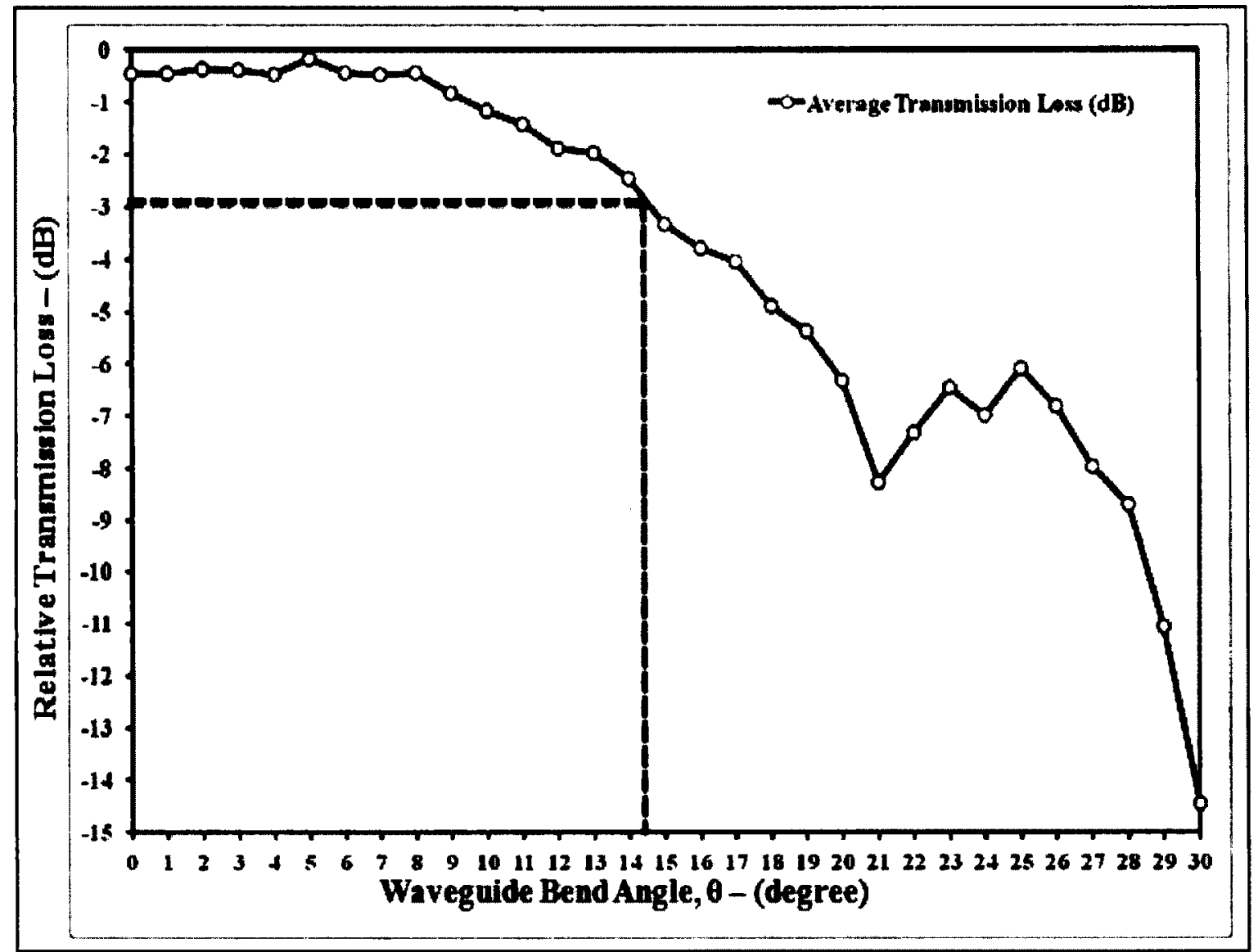

Figure 5.9 (b): Plot showing the average transmission loss for a frequency interval $(8.45 \mathrm{GHz}$ to $8.81 \mathrm{GHz})$ at different waveguide bend angles, $\theta$, varying from $0^{\circ}$ to $30^{\circ}$.

Figure 5.9 (b) shows the average transmission loss of less than $3 \mathrm{~dB}$. up to the waveguide bend angle, $\theta=14^{\circ}$ for a frequency range between $8.45 \mathrm{GHz}$ to $8.81 \mathrm{GHz}$.

The two sets of experimental frequency windows $(7.88 \mathrm{GHz}$ to $8.03 \mathrm{GHz})$ and (8.45 GHz to $8.81 \mathrm{GHz}$ ) were compared with those obtained using FDTD (8.54 GHz to 9.06 GHz). A common set of frequencies $(8.54 \mathrm{GHz}$ to $8.81 \mathrm{GHz}$ ) was selected and considered for further evaluation to find an optimal bend angle to build a $\mathrm{Y}$ branch splitter and see transmission properties at that particular bend angle. 
A 3-D line plot was constructed for a common set of frequencies $(8.54 \mathrm{GHz}$ to $8.81 \mathrm{GHz}$ ) as shown in Figure 5.10 (a) showing the relative transmission loss at different waveguide bend angles $\theta$ varying from $0^{\circ}$ to $30^{\circ}$. This graph shows the transmission behavior at a particular frequency when relative transmission loss increases with the increase of the waveguide bend angle.

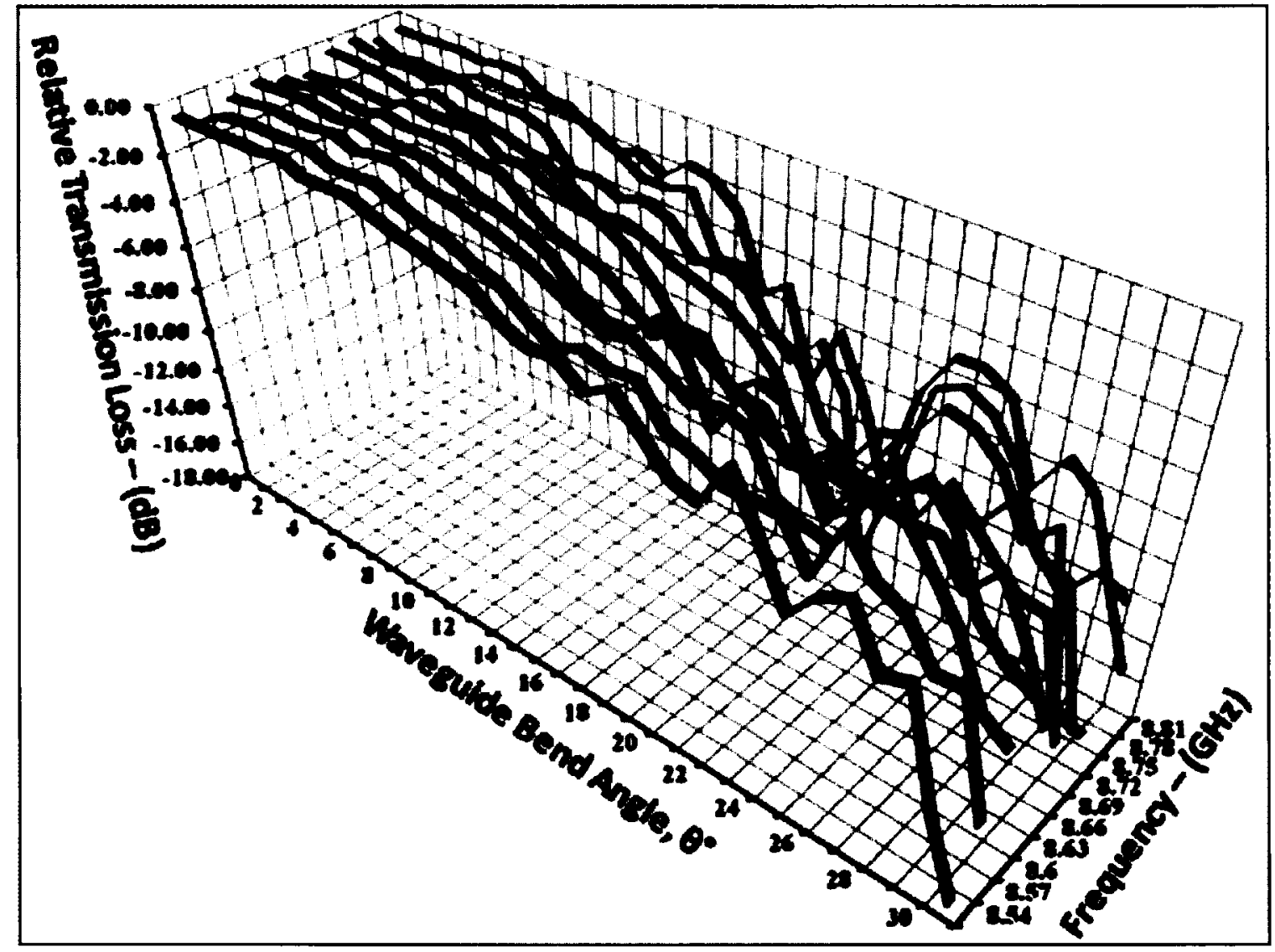

Figure 5.10 (a): 3-D line plot for a frequency range of $8.54 \mathrm{GHz}$ to $8.81 \mathrm{GHz}$ showing a relative transmission loss at different waveguide bend angles, $\theta$, varying from $0^{\circ}$ to $30^{\circ}$.

Figure 5.10 (b) shows a plot between average relative transmission losses versus waveguide bend angle, $\theta$ for a frequency window of $8.54 \mathrm{GHz}$ to $8.81 \mathrm{GHz}$. This graph 
shows the average value of transmission loss at a particular bend angle for a common range of frequencies.

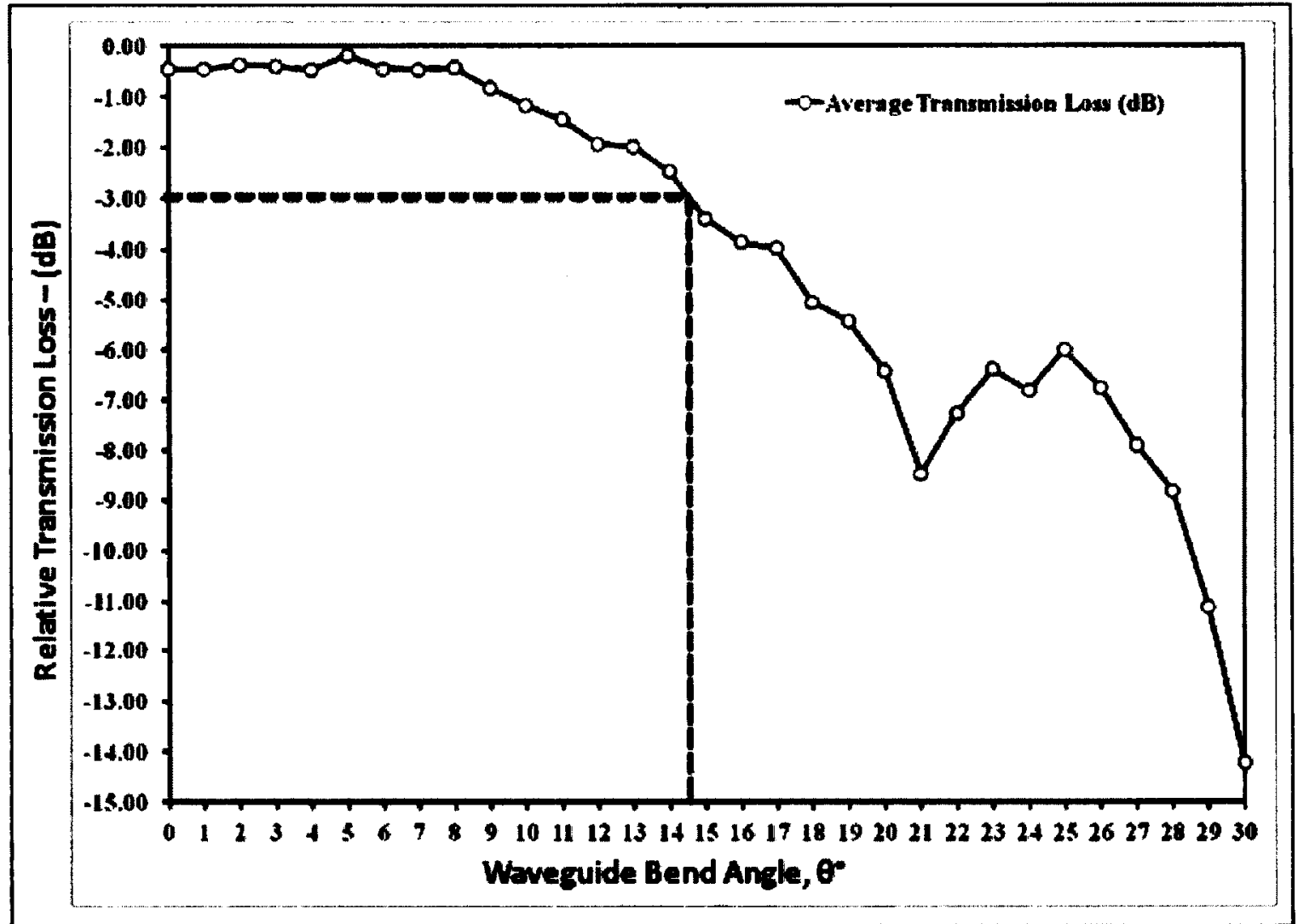

Figure 5.10 (b): Plot showing the average relative transmission loss versus waveguide for a frequency window of $8.54 \mathrm{GHz}$ to $8.81 \mathrm{GHz}$.

Figure 5.11 (a) shows a 3-D line plot showing the transmission efficiency at different waveguide bend angles, $\theta$ for a range of frequency ranging from $8.54 \mathrm{GHz}$ to $8.81 \mathrm{GHz}$. The transmission efficiency at a particular frequency is calculated by taking a ratio between the reference transmission signal (point) at a particular angle, $\theta$ to the transmission signal (point) at that particular angle. 


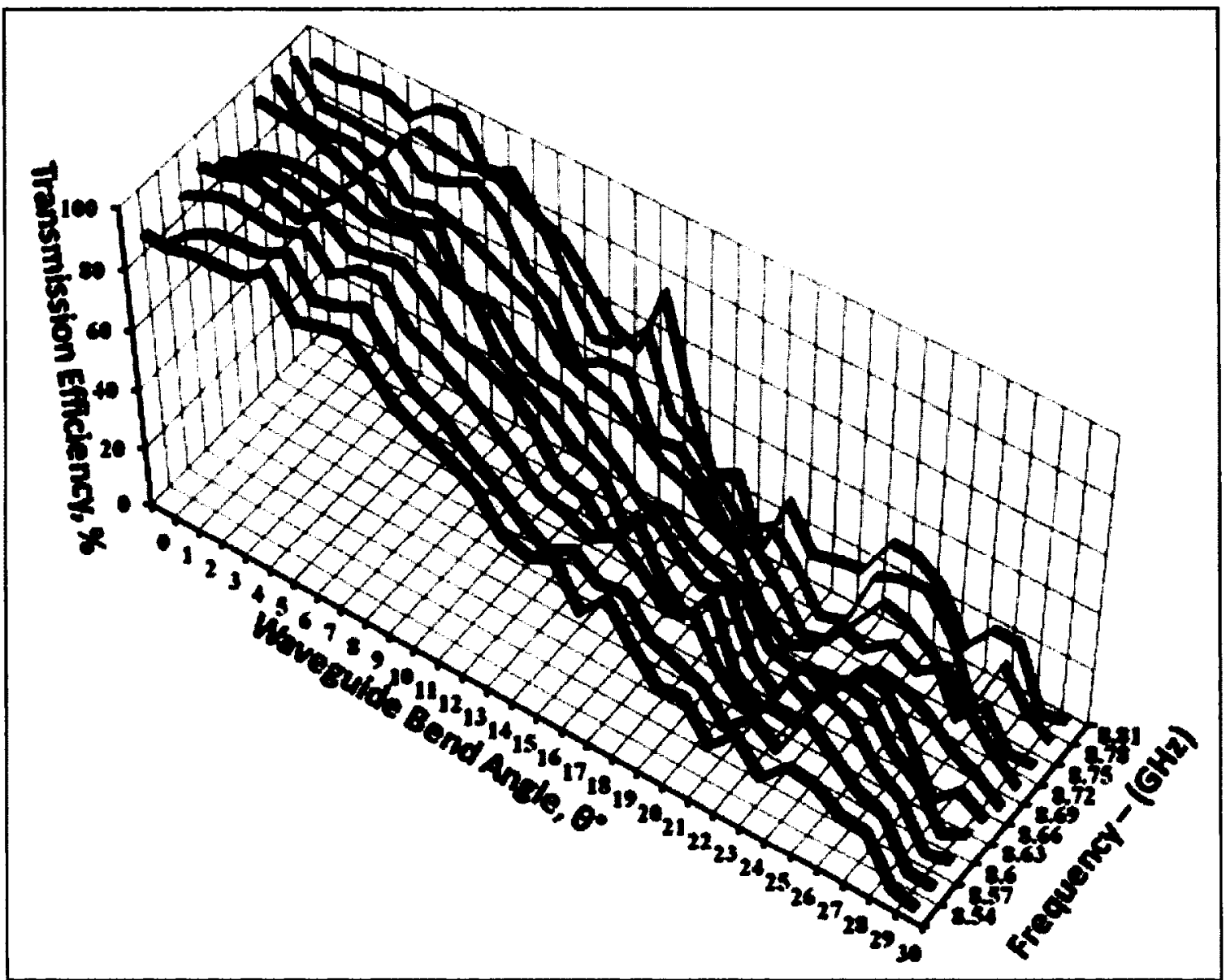

Figure 5.11 (a): 3-D line graph showing transmission efficiency at different waveguide bend angles, $\theta$ for a range of frequency $(8.54 \mathrm{GHz}$ to $8.81 \mathrm{GHz})$.

Figure 5.11 (b) shows the average transmission efficiency over a range of frequencies $(8.54 \mathrm{GHz}$ to $8.81 \mathrm{GHz})$ at different waveguide bend angles, $\theta$ varying from $0^{\circ}$ to $30^{\circ}$. Average transmission efficiency is calculated by taking the average of the transmission efficiency at a particular bend angle for a chosen frequency interval. 


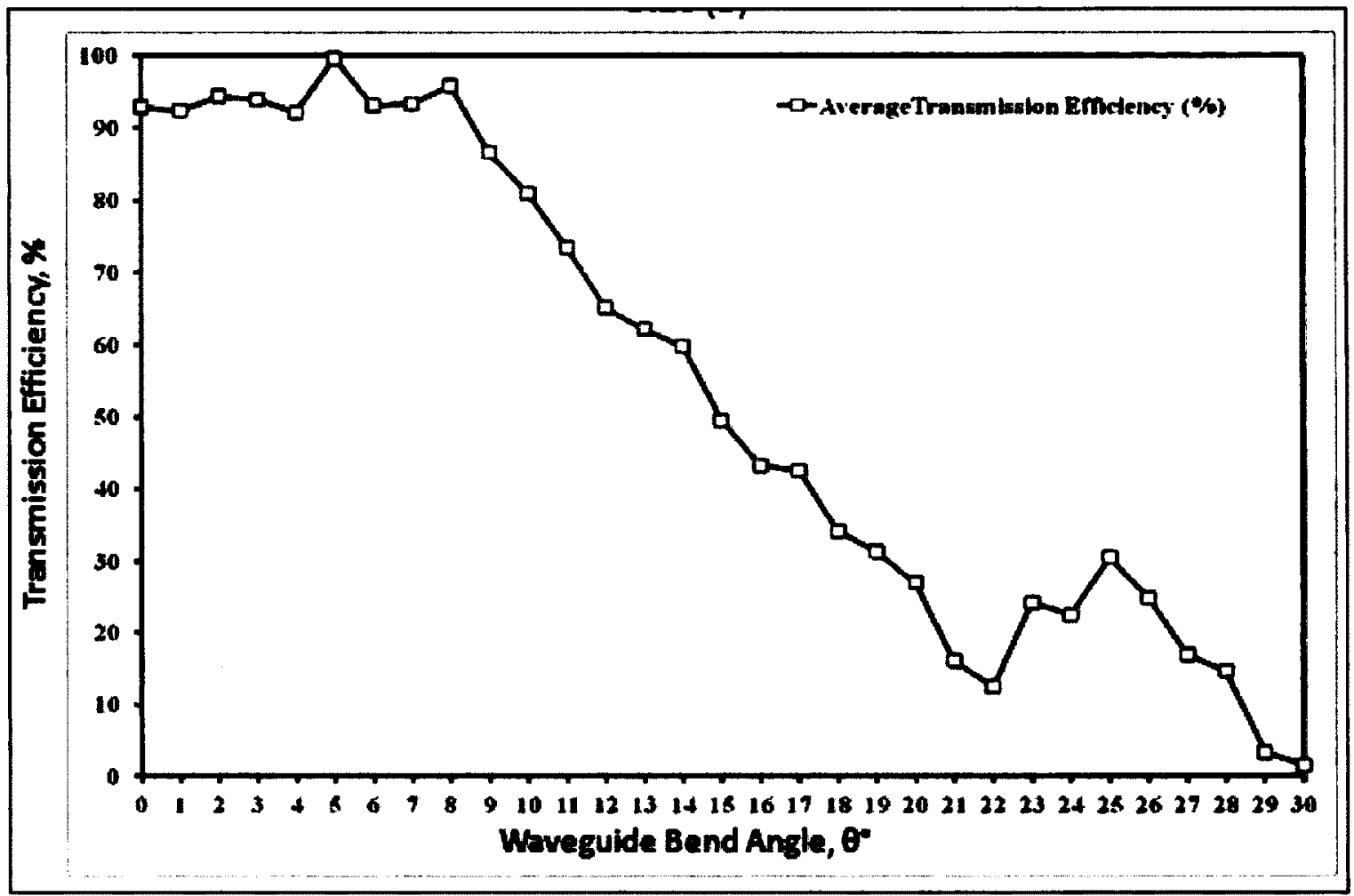

Figure 5.11 (b): Plot showing the average transmission efficiency for a frequency range ( $\mathrm{f}=8.54 \mathrm{GHz}$ to $8.81 \mathrm{GHz})$ at different waveguide bend angles $\left(\theta=0^{\circ}\right.$ to $\left.30^{\circ}\right)$ that are guided through a 2-D photonic crystal linear waveguide in the microwave regime.

From Figure 5.10 (b) and Figure 5.11 (b), the following observations were made:

i. For waveguide bend angle, $\left(\theta=0^{\circ}\right.$ to $\left.8^{\circ}\right)$, the relative transmission efficiency is near or above $95 \%$ with an average relative transmission loss of $0.40 \mathrm{~dB}$.

ii. For waveguide bend angle, $\left(\theta=9^{\circ}\right.$ to $\left.15^{\circ}\right)$, the relative transmission efficiency decreased to $70 \%$ with an average relative transmission loss of $1.88 \mathrm{~dB}$.

iii. For waveguide bend angle, $\left(\theta=16^{\circ}\right.$ to $\left.20^{\circ}\right)$, the relative transmission efficiency rapidly decreased to $35 \%$ with an average relative transmission loss of $4.95 \mathrm{~dB}$. 
iv. For waveguide bend angle, $\left(\theta=21^{\circ}\right.$ to $\left.22^{\circ}\right)$, the relative transmission efficiency rapidly decreased to $14 \%$ with an average relative transmission loss of $7.87 \mathrm{~dB}$.

v. For waveguide bend angle, $\left(\theta=23^{\circ}\right.$ to $\left.25^{\circ}\right)$, the relative transmission efficiency improved and reached $25 \%$ with an average relative transmission loss of $6.4 \mathrm{~dB}$. This occurred due to the inclined waveguide forming a cavity between bends and acting as a resonator.

vi. For waveguide bend angle, $\left(\theta=26^{\circ}\right.$ to $\left.30^{\circ}\right)$, the relative transmission efficiency decreased gradually to $0 \%$ with an average relative transmission loss of $9.75 \mathrm{~dB}$.

From these observations, a range of waveguide offset angles, $\theta$ was considered from $0^{\circ}$ to $15^{\circ}$ because, as the waveguide offset angle increased beyond $15^{\circ}$, the relative transmission efficiency decreased below $50 \%$ and relative transmission loss became more than $3 \mathrm{~dB}$.

A plot as shown in Figure 5.12 was also drawn at a particular frequency point, $f=$ 8.54 GHz with different waveguide bend angles, $\theta$ versus transmission loss. This frequency was chosen from a set of frequencies $(\mathrm{f}=8.54 \mathrm{GHz}$ to $8.81 \mathrm{GHz}$ ) that have been observed theoretically as well as experimentally, and proven to have high transmission efficiency and low transmission loss. This figure illustrates the effect of waveguide bend angles on transmission loss. There are a few peaks at $\theta=22^{\circ}, 26^{\circ}$ where transmission loss tries to decrease. This is because of the resonator formed at the bends of the waveguide. Different plots showing a set of frequencies ( $\mathrm{f}=8.57 \mathrm{GHz}$ to $8.81 \mathrm{GHz}$ ) 
with different waveguide bend angles, $\theta$ versus relative transmission loss in microwave regime are shown in Appendix D.

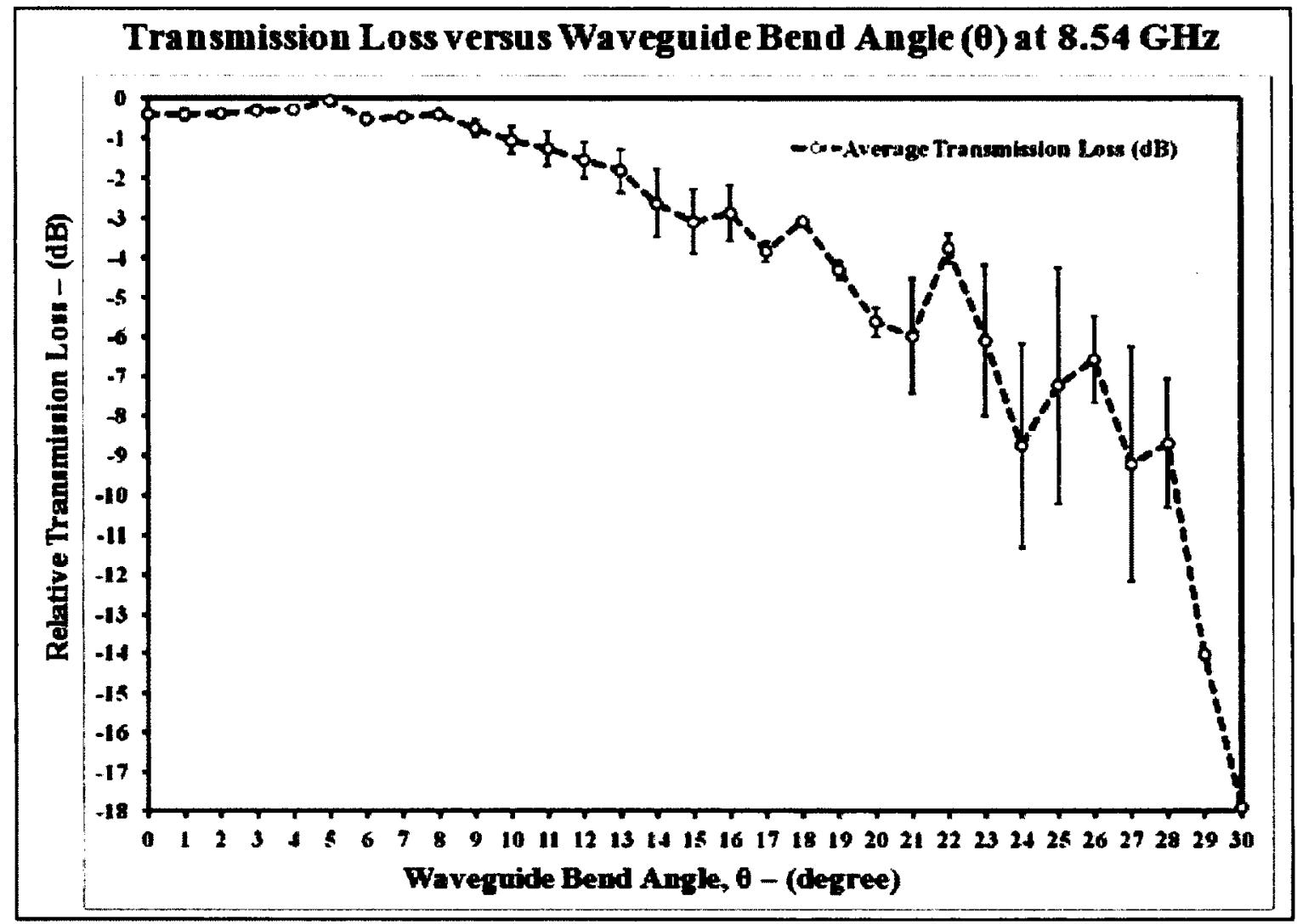

Figure 5.12: Plot relative transmission loss versus waveguide bend angle for the frequency of $8.54 \mathrm{GHz}(\lambda$ $=1.45 \mu \mathrm{m})$

\subsection{Summary of Theoretical and Experimental Results for a Linear Waveguide}

As previously discussed, while performing the FDTD simulation and microwave experiment, a range of waveguide bend angles, $\theta$, were considered from $0^{\circ}$ to $30^{\circ}$. After running the FDTD simulation and performing experimental measurements at a particular frequency, $8.54 \mathrm{GHz}$ (corresponding to wavelength, $\lambda=1.45 \mu \mathrm{m}$ ), a plot was created 
between transmission loss and waveguide bend angle, $\theta$, both for the theoretical and experimental measurements as shown in Figure 5.13.

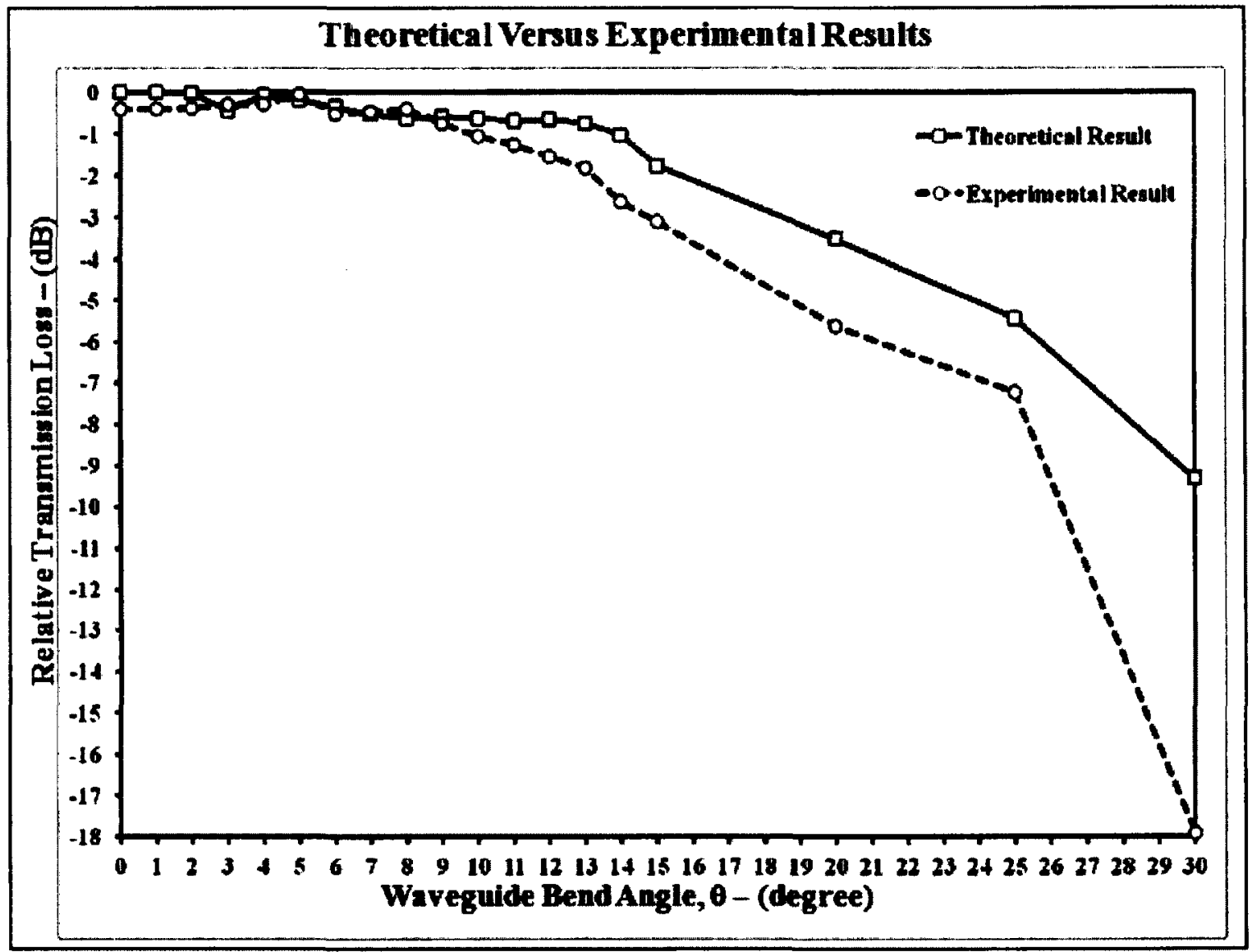

Figure 5.13: Plot of relative transmission loss versus waveguide bend angle for the theoretical predictions and experimental results at a frequency of $8.54 \mathrm{GHz}(\lambda=1.45 \mu \mathrm{m})$.

Both theoretical and experimental results show a very close relation at waveguide bend angle, $\theta=5^{\circ}$ with a percentage error of $1.4 \%,\left[\left(\frac{\mathrm{P}_{\mathrm{T}}-\mathrm{P}_{\mathrm{E}}}{\mathrm{P}_{\mathrm{E}}}\right) \times 100 \%\right]$ where, $\mathrm{P}_{\mathrm{T}}=$ transmitted power (theoretical value) and $P_{E}=$ transmitted power (experimental value). 
This waveguide bend angle at a frequency $(\mathrm{f}=8.54 \mathrm{GHz})$ with low transmission loss is well-suited to design and fabricate a Y branch splitter in the microwave regime.

\subsection{Y splitter}

Having shown that the dielectric shift process can produce low loss bends in the photonic crystal waveguide, the process is applied in the production of a Y branch splitter. In this structure, shown in Figure 5.14, the bend region dielectric is horizontally divided at the center of the waveguide. The dielectric is incrementally shifted upwards above the center line and downwards below the center line. When possible, additional rods are inserted to complete the triangular array and define the bend waveguide section. The two resulting waveguides are then terminated with parallel and well-separated waveguides that serve as the outputs. 


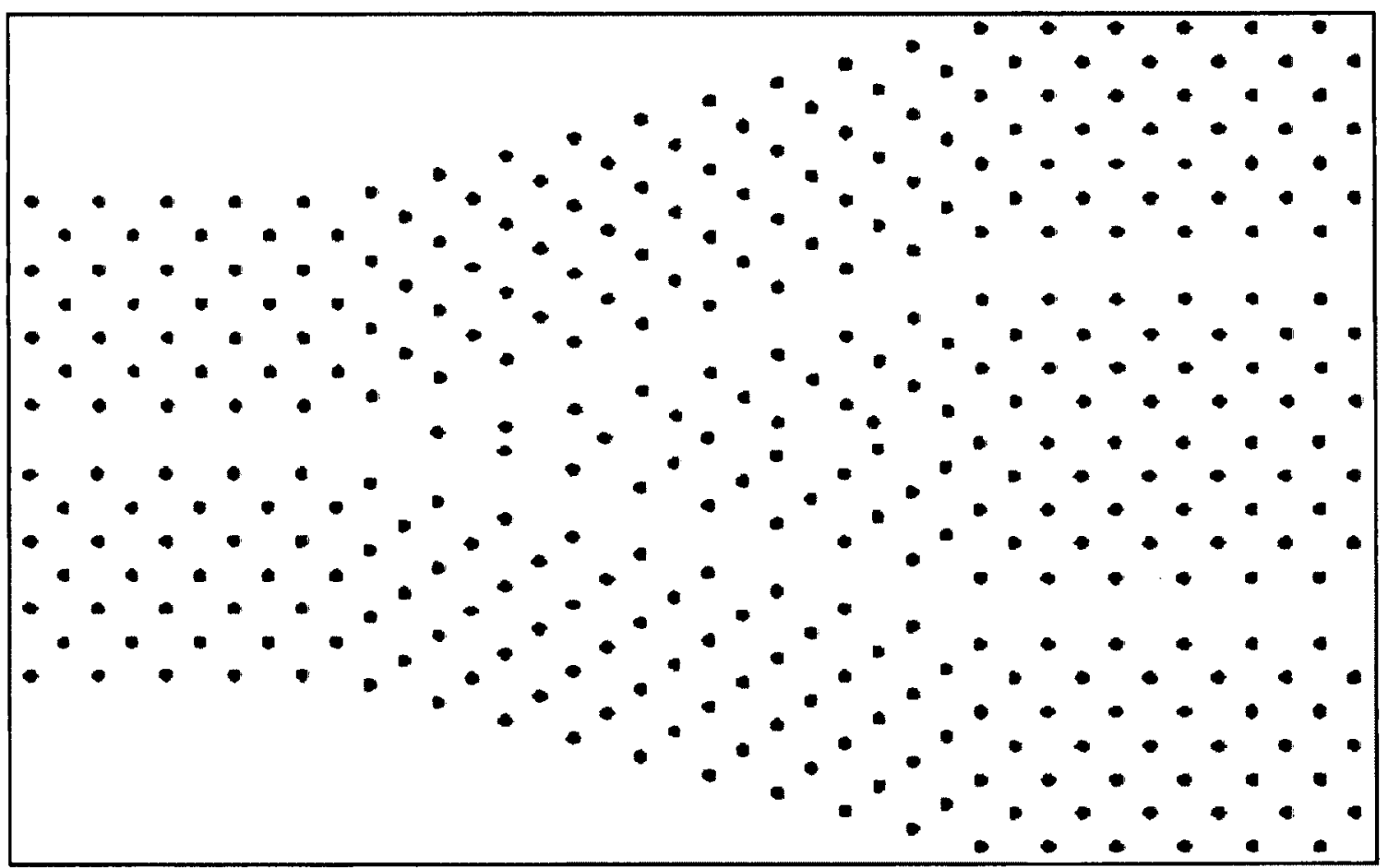

Figure 5.14: Sketch of the $\mathrm{Y}$ branch splitter produced using the increment dielectric shift process.

A Y branch consisting of 1-2 splitter with overall dimensions of $299.1 \mathrm{~mm} \mathrm{X}$ $262.45 \mathrm{~mm}$ as shown in Figure 5.15 was constructed at an angle $\left(\theta=5^{\circ}\right)$ from the same 2-D photonic crystal plexi-glass template. 


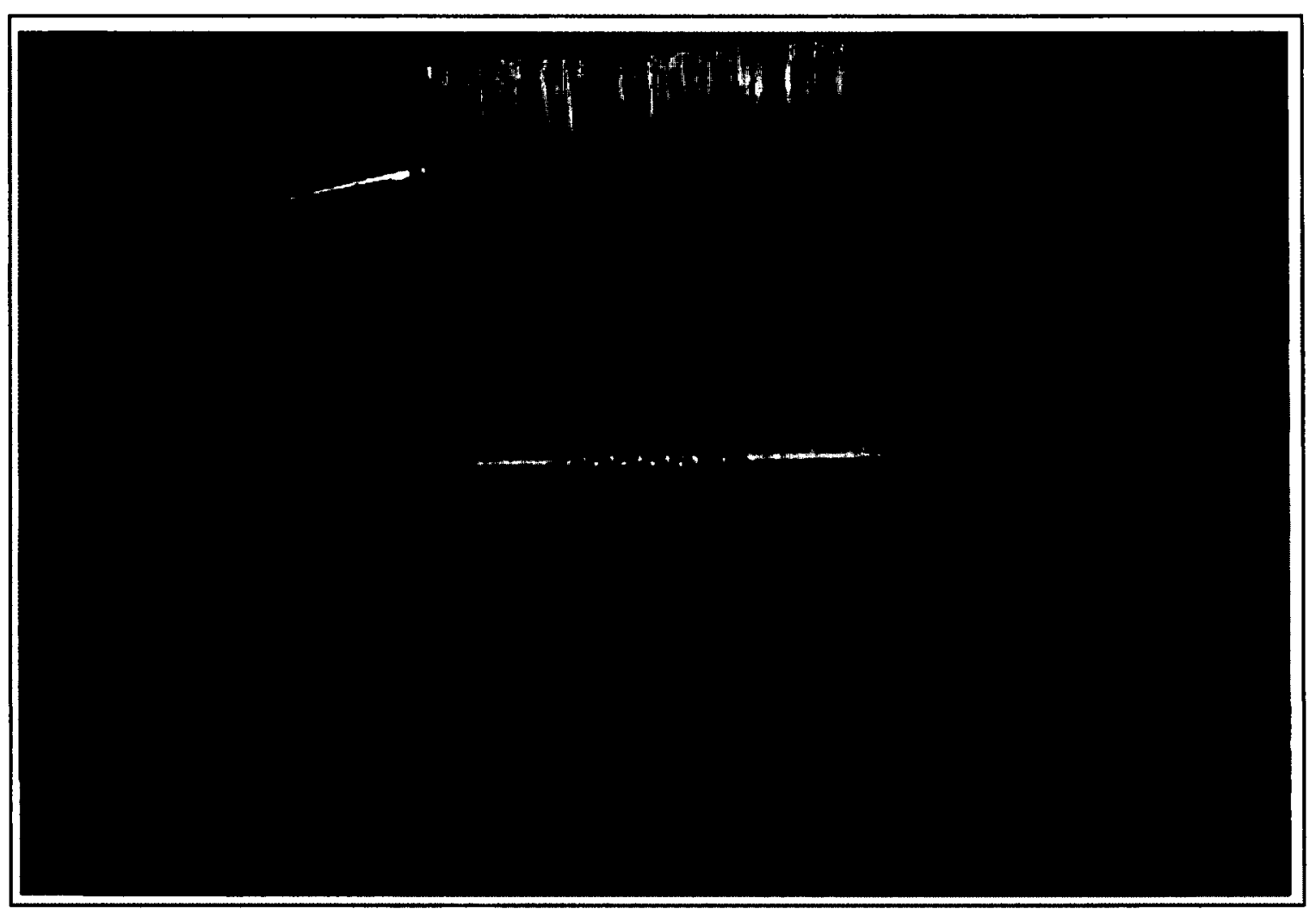

Figure 5.15: A complete microwave experimental setup (side view) of 2-D Y branch (1 X 2 splitter) model with triangular lattice with overall dimensions of $299.1 \mathrm{~mm} \mathrm{X} 262.45 \mathrm{~mm}$ accommodating 234 alumina rods.

A few alterations were made to section 2 (moving part-strip type 1 and 2) of the linear waveguide template by trimming the strip edges on both sides so that the strips could be connected end to end to form a $\mathrm{Y}$ branch. It contains 17 periods in the $\mathrm{Y}$ direction and 31 periods (9-input, 13 - middle, 9 - output) in the $\mathrm{X}$ direction.

The transmitter was kept at the starting edge of the array at the fixed position and the receiver was kept at the end of the structure where it receives the output signal from either output splitter branch as shown in Figure 5.16. The $S_{21}$ parameter was measured 
with the help of a network analyzer and transmission curves were plotted for both output signals received.

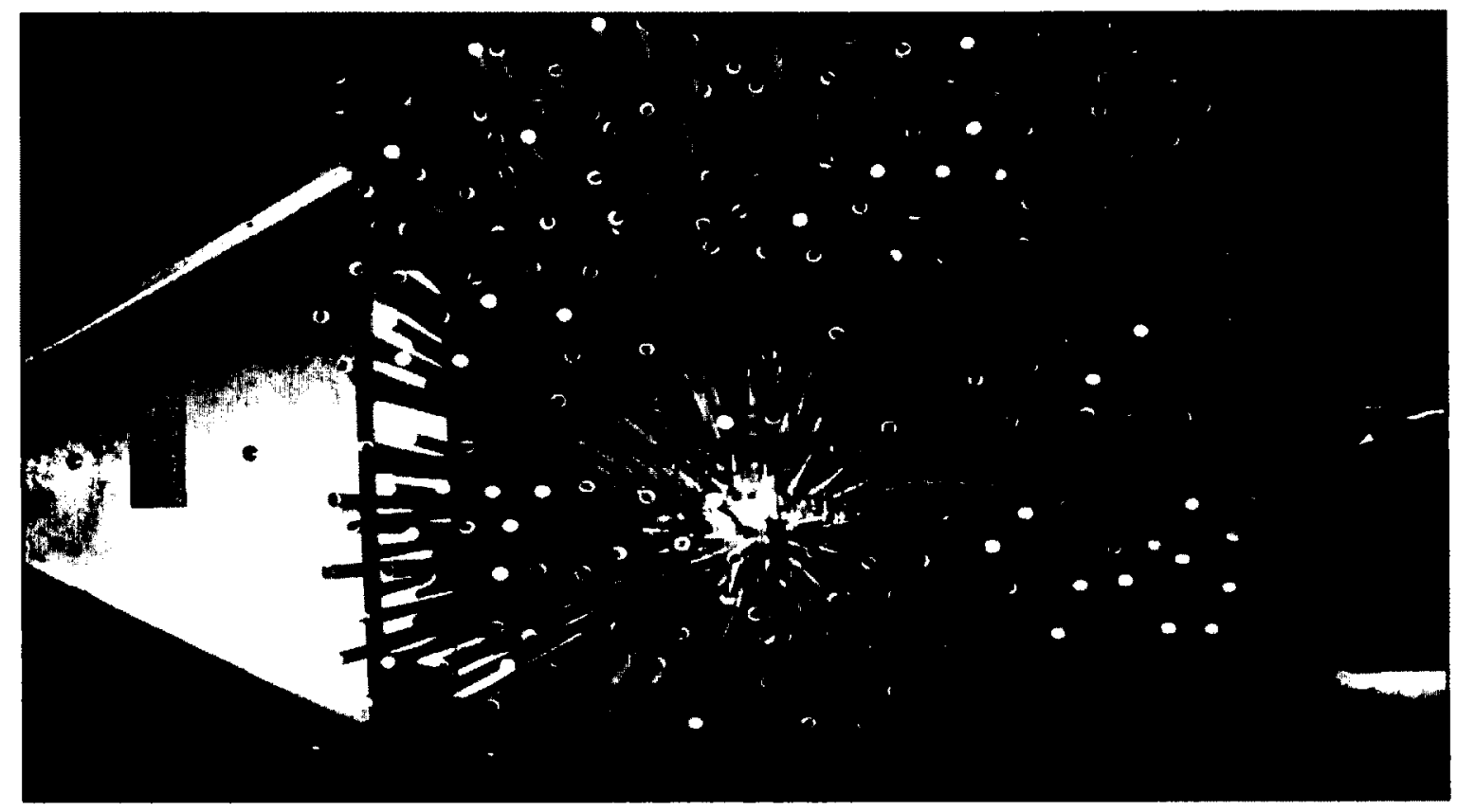

Figure 5.16: Top view of the 2-D photonic crystal Y splitter structure (triangular lattice) with one input and two outputs.

Figure 5.17 shows a transmission spectrum when the bend angle for each waveguide in the $\mathrm{Y}$ branch is $5^{\circ}$. These measurements were obtained for the TM polarization. The overall transmission power drop was $-20 \mathrm{~dB}$. The range of frequency guided through this $\mathrm{Y}$ splitter at two outputs is from $7.73 \mathrm{GHz}$ to $8.81 \mathrm{GHz}$ with a bandwidth of $1.08 \mathrm{GHz}$. 


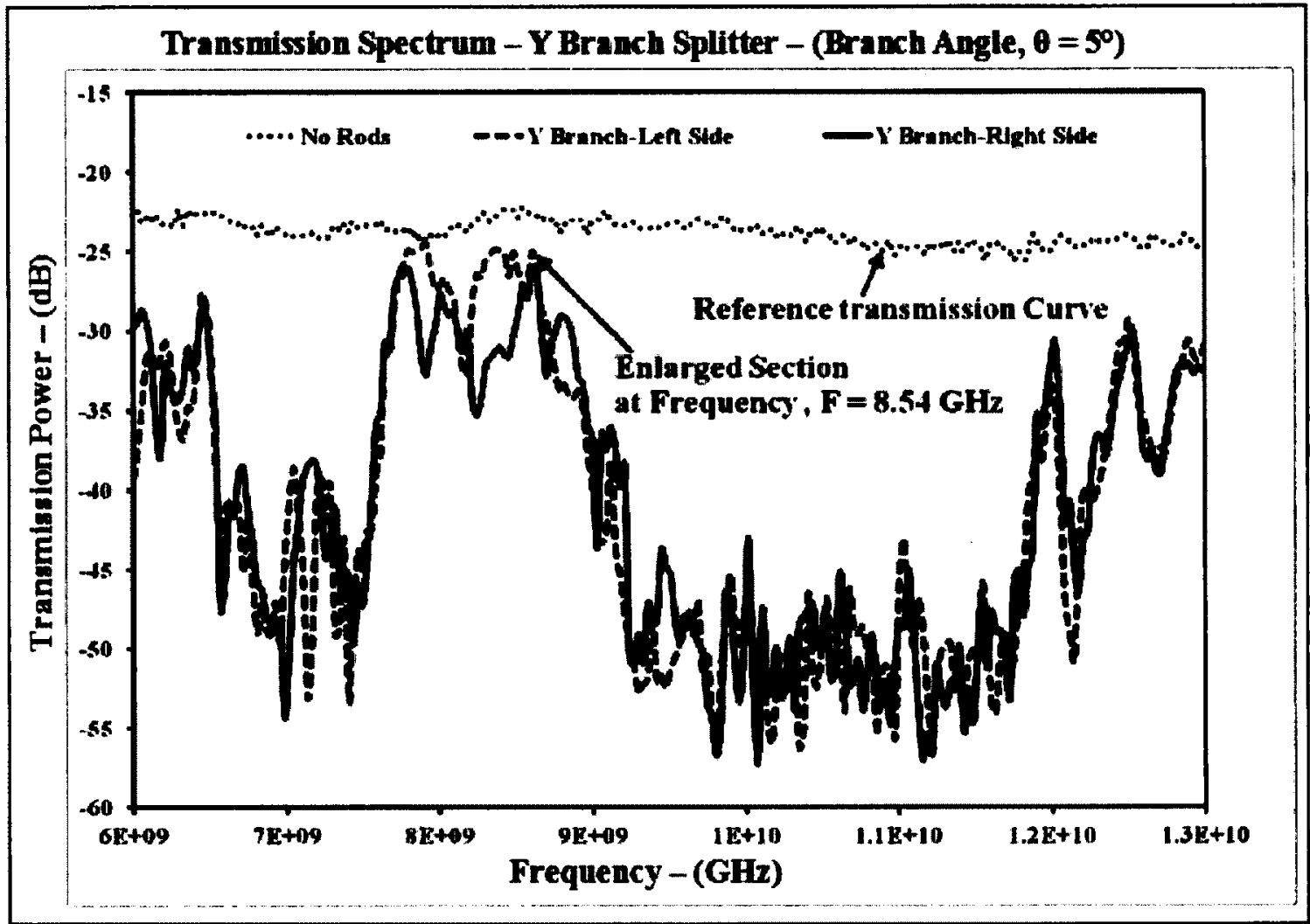

Figure 5.17: Transmission spectrum for a $Y$ splitter designed with a branch angle, $\theta=5^{\circ}$, in the microwave regime. The split ratio is nearly $50 / 50$ with an overall efficiency of $82 \%$

Figure 5.18 shows the enlarged section of the transmission spectrum for a $\mathrm{Y}$ splitter as shown in Figure 5.17. From this spectrum, an observation was made at frequency, $f=8.54 \mathrm{GHz}$. While observing this particular frequency, a conclusion was drawn that the split is nearly $50 / 50$ with an overall transmission efficiency of $82 \%$ as compared to the output transmission efficiency of a linear waveguide at $\theta=5^{\circ}$ at $98 \%$ with percentage difference of $16 \%$. There is equal distribution of the input signal in the Y splitter with the output efficiency of each branch at $41 \%$. So, last but not the least, it was concluded that the transmission loss through the $\mathrm{Y}$ branch splitter is below $1 \mathrm{~dB}$ and 
transmission efficiency is still sufficiently high to consider this structure to be designed in the optical regime.

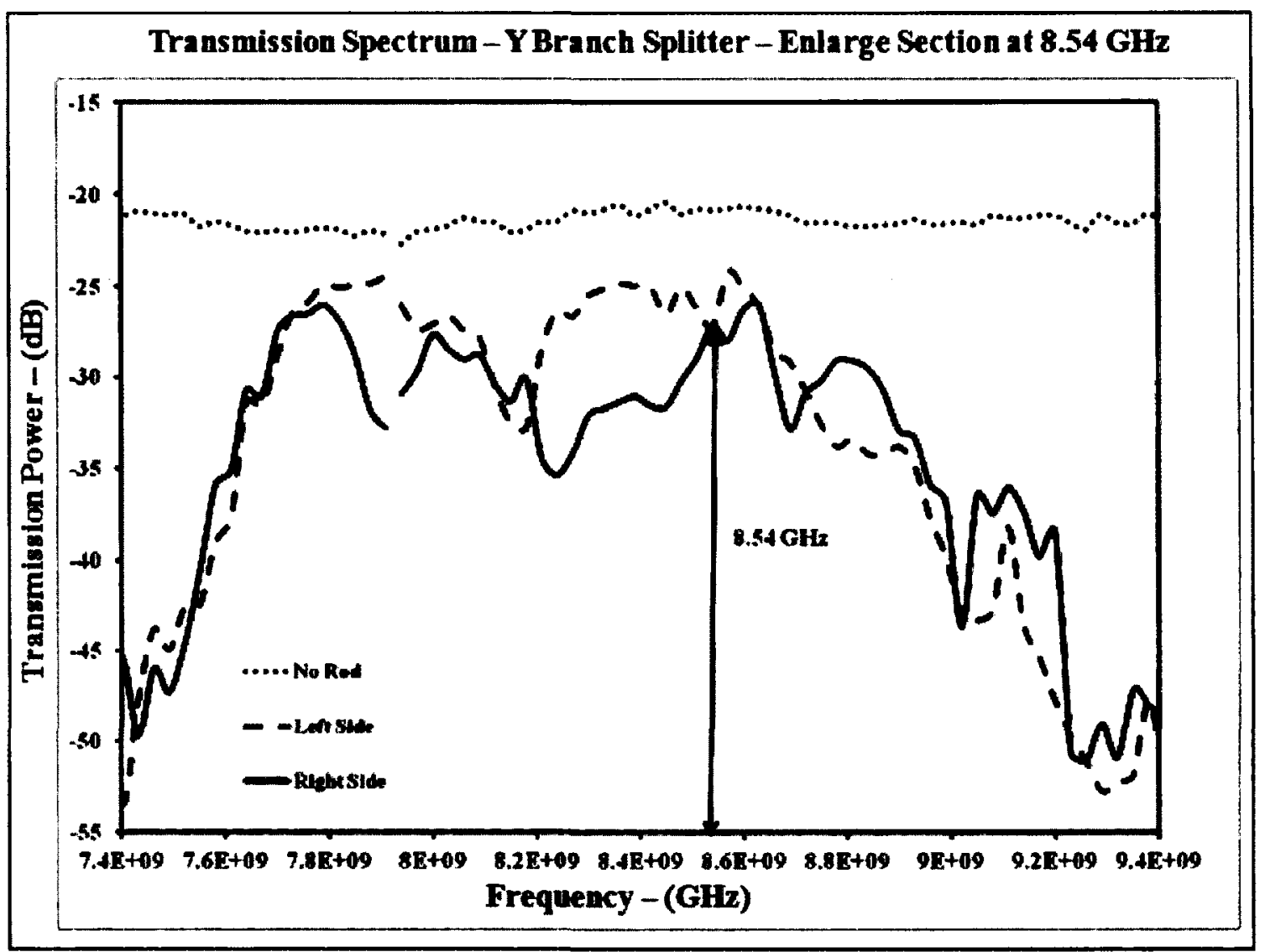

Figure 5.18: Enlarged section (Figure (5.17)) of the transmission spectrum for a $\mathrm{Y}$ splitter at frequency, $\mathrm{f}=$ $8.54 \mathrm{GHz}$ showing two output waveguides with transmission efficiency of $41 \%$ each. 


\section{Conclusions}

The main focus of this thesis was to investigate low loss waveguide bends using a dielectric shift technique to introduce the bends in a photonic crystal waveguide. This investigation led to the designing of a low loss $\mathrm{Y}$ splitter examined in the microwave regime. A summary of the findings is listed below:

i. The plane wave and FDTD methods were used to evaluate 2-D photonic crystal band structures for the TM polarization. A suitable triangular lattice composed of high dielectric rods in air showed a bandgap in the normalized frequency range of 0.36 to 0.55 which corresponds to the $6.5 \mathrm{GHz}$ to $9.80 \mathrm{GHz}$ frequency range in the microwave regime.

ii. In the obtained structure, a 2-D photonic crystal linear waveguide was designed by removing one row of rods in the propagation direction (X). After analyzing theoretical and experimental results, a common range of frequency, $(8.54 \mathrm{GHz}$ to 8.81 GHz) showing high transmission efficiency was considered for further waveguide bend loss investigation.

iii. A plexi-glass template was constructed to hold several hundred alumina rods in a triangular lattice. The template permitted the microwave regime analysis of the waveguide when subjected to the bending. Using a dielectric shift technique, the central portion of the waveguide contained two bends in which the bend angle ranged from $0^{\circ}$ to $30^{\circ}$. It was found that low loss bends of less than $3 \mathrm{~dB}$ are obtained for bend angles of less than $15^{\circ}$. This investigation led to choosing a 
particular waveguide bend angle, $\theta$ as $5^{\circ}$ at which there is a single guided wavelength, $\lambda=1.45 \mu \mathrm{m}$ corresponding to frequency, $\mathrm{f}=8.54 \mathrm{GHz}$ through a waveguide channel showing a higher transmission efficiency (97\%).

iv. This conclusion narrows the path towards one particular direction for designing and fabricating a $\mathrm{Y}$ splitter at a branch angle, $\theta=5^{\circ}$ using the same template with few modifications. The overall transmission efficiency of $82 \%$ with $50 / 50$ split over each branch as compared to the output transmission efficiency of a linear waveguide at $\theta=5^{\circ}$ of $98 \%$ with percentage error of $16 \%$.

With this optical design, one can design these structures in optical regimes that solves the problem of high power dissipation in optic circuits. Also, many components can be interconnected with each other to perform high-speed functions and processes.

\subsection{Potential Application of Y branch Splitter}

The $\mathrm{Y}$ branch splitter finds a potential application in various optoelectronic waveguide devices, such as directional coupler, Mach-Zehnder, switching and optical sensors $^{26,27,39,40,41,42,43,44,45,46}$. 


\subsection{Future Directions}

This thesis was concerned only with the TM polarization. Future experiments can examine the transmission of the TE polarization in asuitable photonic crystal and design low loss bends using the same dielectric shift procedure. Structures can also be extended to include confinement in the out-of-plane direction, resulting in 3-D photonic crystal waveguides with bends. A natural extension of this work is to build and test the structures in the optical domain. 


\section{Appendix A :}

Complete Photonic Bandgap Maps (TM and TE polarization) 


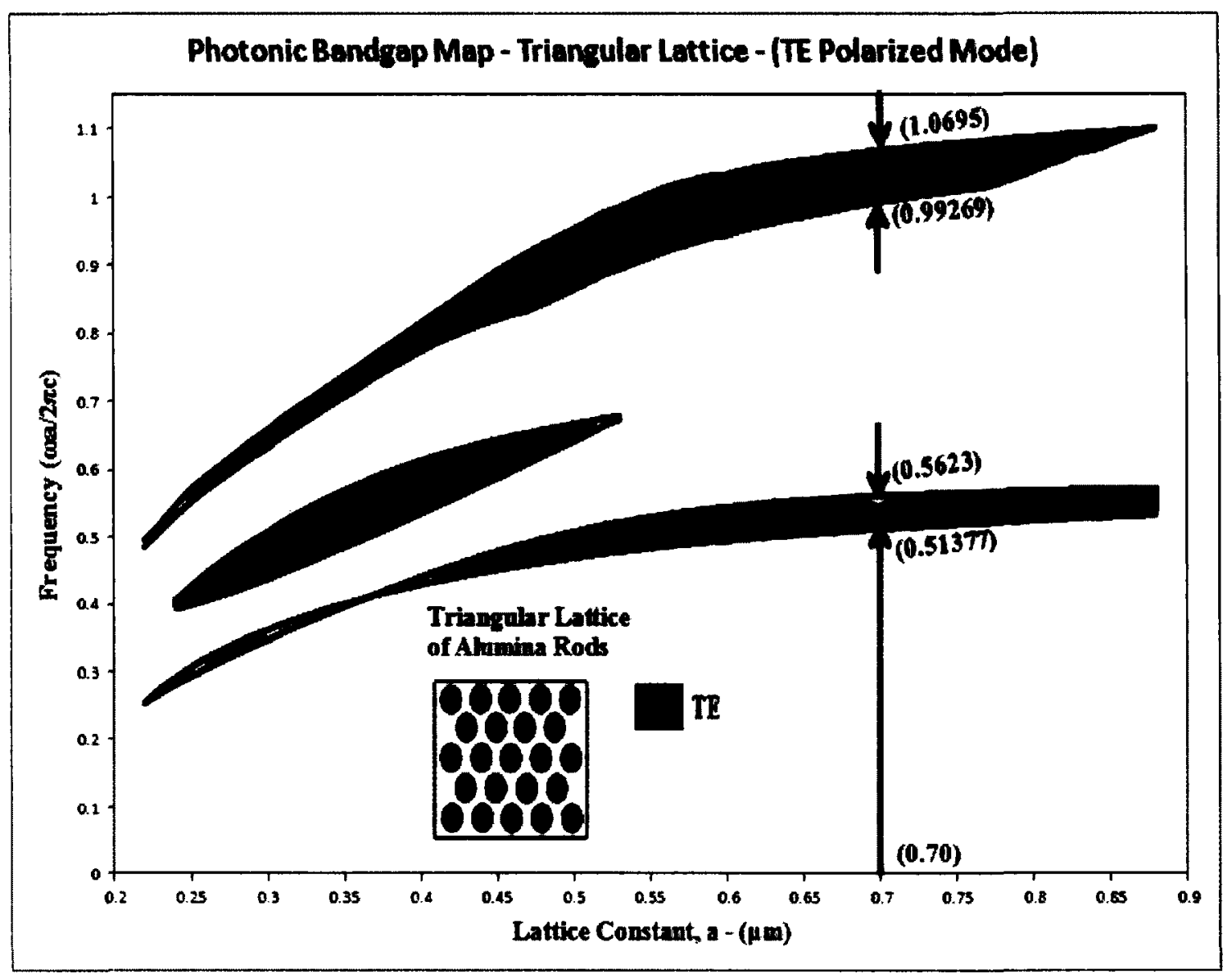

Figure (A.1): Plot of normalized frequency, $\left(\frac{\omega a}{2 \pi c}\right)$ versus lattice constant ' $a$ '. Photonic bandgaps are shown for a two dimensional photonic crystal (TE polarization) with triangular lattice of dielectric rods $\left(\varepsilon_{\mathrm{Al}}=9.2\right)$ with a background as air $\left(\varepsilon_{\text {air }}=1\right)$. The inset shows the triangular lattice formations of dielectric alumina rods. Rod radius is constant, ' $r=0.1 \mu \mathrm{m}$ ' and expected value of chosen lattice constant, ' $a=0.70$ $\mu \mathrm{m}$ ' corresponding to TM polarization. 


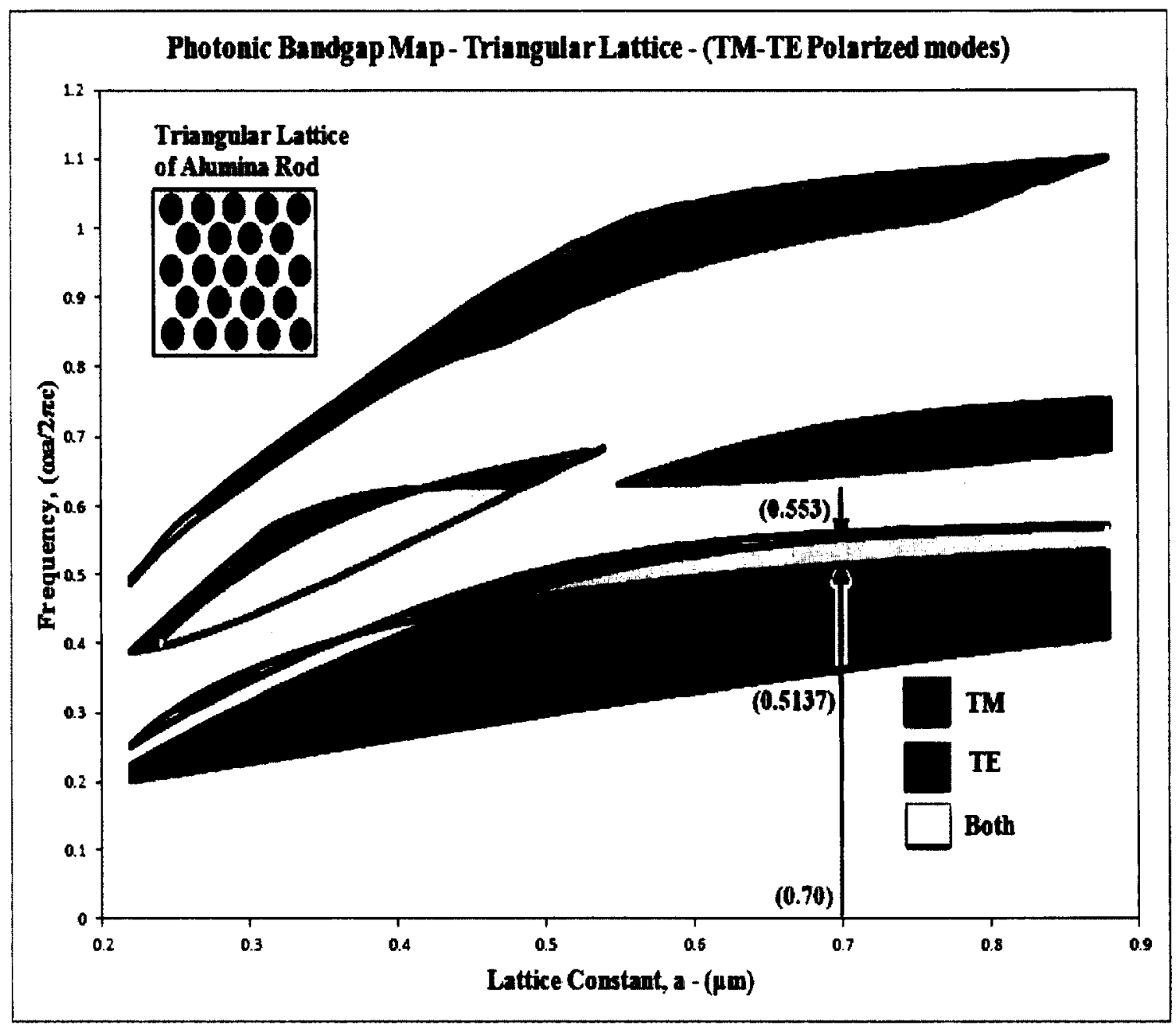

Figure (A.2): Plot of normalized frequency, $\left(\frac{\omega a}{2 \pi c}\right)$ versus lattice constant ' $a$ '. A complete photonic bandgap for a two dimensional photonic crystal (TM and TE polarization) with triangular lattice of dielectric rods $\left(\varepsilon_{\mathrm{Al}}=9.2\right)$ with background as air $\left(\varepsilon_{\text {air }}=1\right)$. The inset shows the triangular lattice formations of dielectric alumina rods. Rod radius is constant, ' $r=0.1 \mu \mathrm{m}$ ' and expected value of chosen lattice constant, ' $a=0.70$ $\mu \mathrm{m}$ ' corresponding to a complete band gap for both TE and TM modes. 


\section{Appendix B:}

Data Sheet for Alumina Rods Showing Physical, Mechanical, Thermal and Electrical Properties 


\section{AL UMINA}

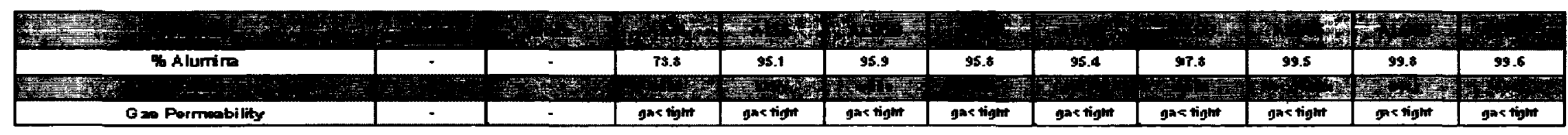

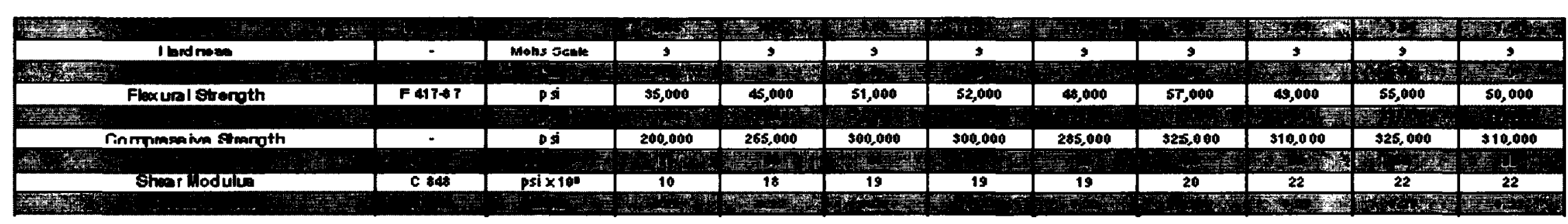

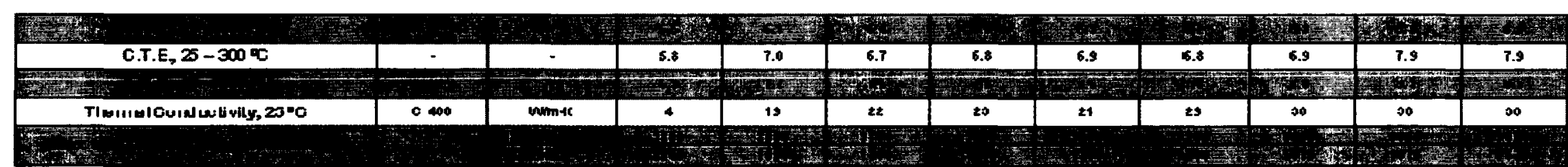

\begin{tabular}{|c|c|c|c|c|c|c|c|c|c|c|c|}
\hline Dialect is ofrergth $\left(12^{\prime \prime}\right.$ thich & D 1AGATA & Wtmil & 225 & 250 & 250 & 250 & 250 & 260 & 270 & 290 & 255 \\
\hline Uieloctric Lionatant & & & nth & 9.2 & 9.0 & 9.1 & 8 & 9.4 & 9.7 & 10.0 & net \\
\hline GE & 0 2520-55 & & $n$ & Q11n & $\operatorname{\theta in}^{\ln k}$ & Q1na & Oin $*$ & Q0: & 0.2 & Da & $n$ \\
\hline Dielectrio Loes & 0.2530 .9 & & $\mathrm{nt}$ & 0.0009 & 0.0007 & 0.0007 & 0.0006 & 0.0000 & $<0.0000$ & $<0.0001$ & nat \\
\hline gork & $0.020 \theta-30$ & & int & 0.12 .5 & 810.6 & 国10.3 & 0.010 .6 & 잉.9.0 & 89.6 & 00.6 & int \\
\hline Yolume Pesietivity, $300^{\circ} \mathrm{C}$ & & & $A E+1$ & $S E+12$ & $1 E+12$ & $3 E+12$ & $1 E+11$ & $B E=1$ & $1 E+15$ & $3 E+12$ & nim \\
\hline
\end{tabular}

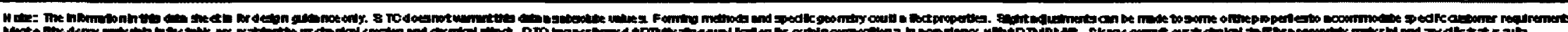

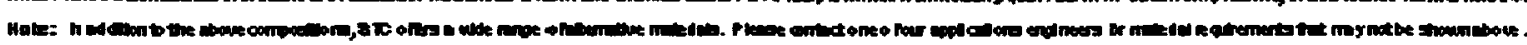

rmestox: insern 11 SUPERIOR TE CTINICAL CRRAMTCS CORP

Figure B.1: Brief data sheet for different alumina rod composition prepared by Superior Technical Ceramics corp. 


\section{Appendix C:}

Experimental Results for Linear Waveguide at Different Waveguide Bend Angle, $\theta$ 


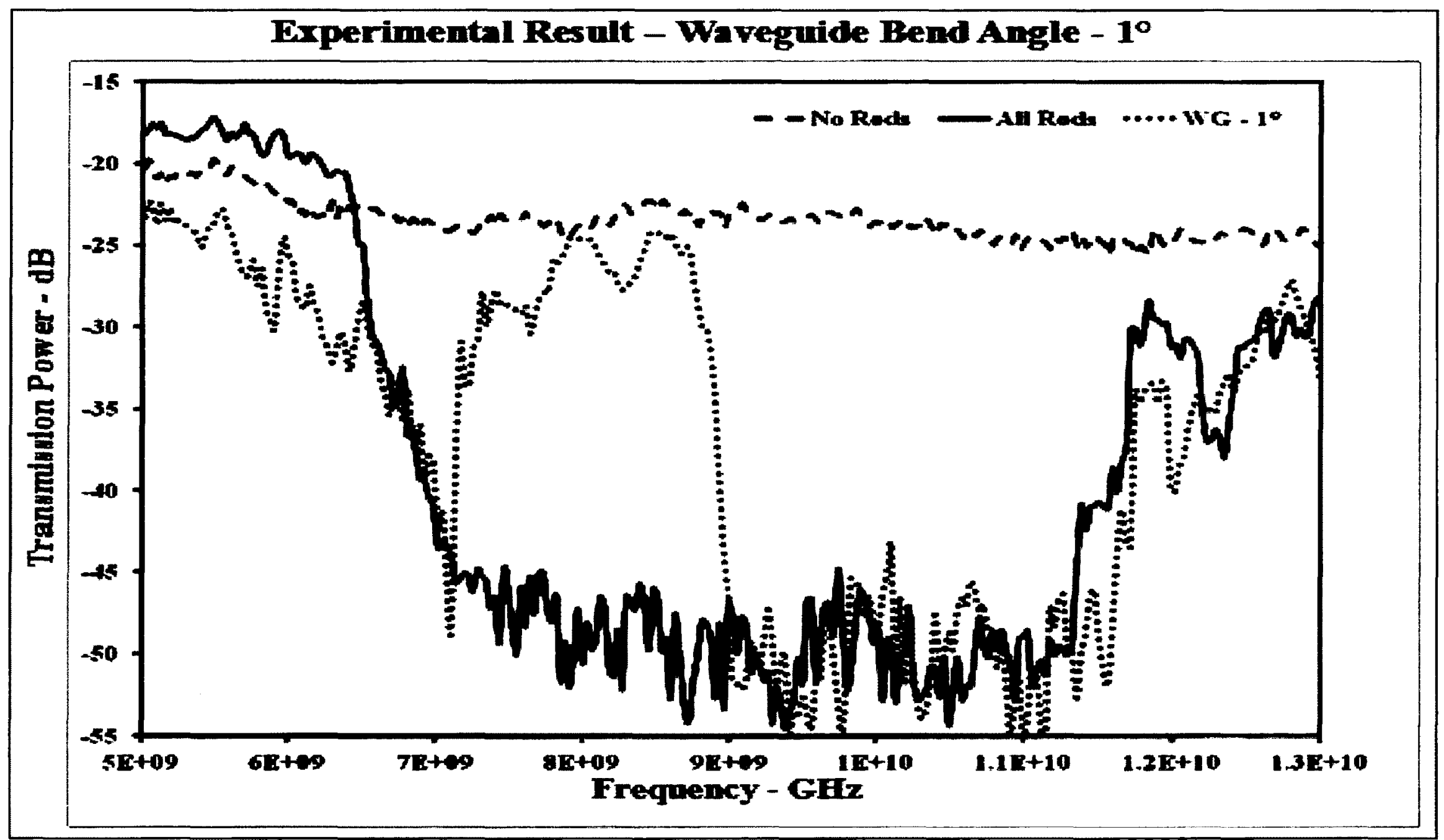

Figure C.1: Transmission spectrum for a linear waveguide with lattice constant, $a=16.9 \mathrm{~mm}$ at bend angle, $\theta=1^{\circ}$ 


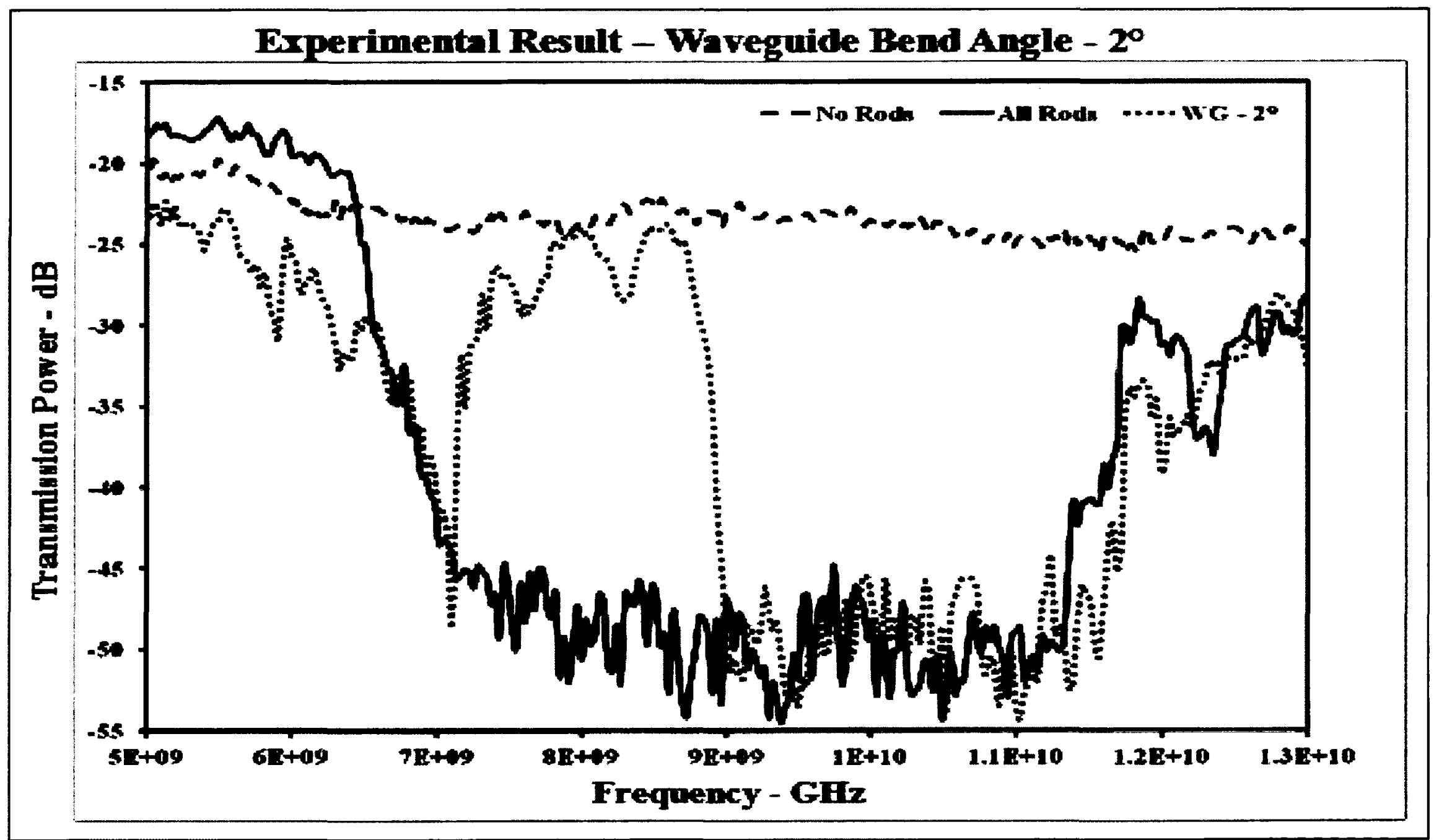

Figure C.2: Transmission spectrum for a linear waveguide with lattice constant, $a=16.9 \mathrm{~mm}$ at bend angle, $\theta=2^{\circ}$ 


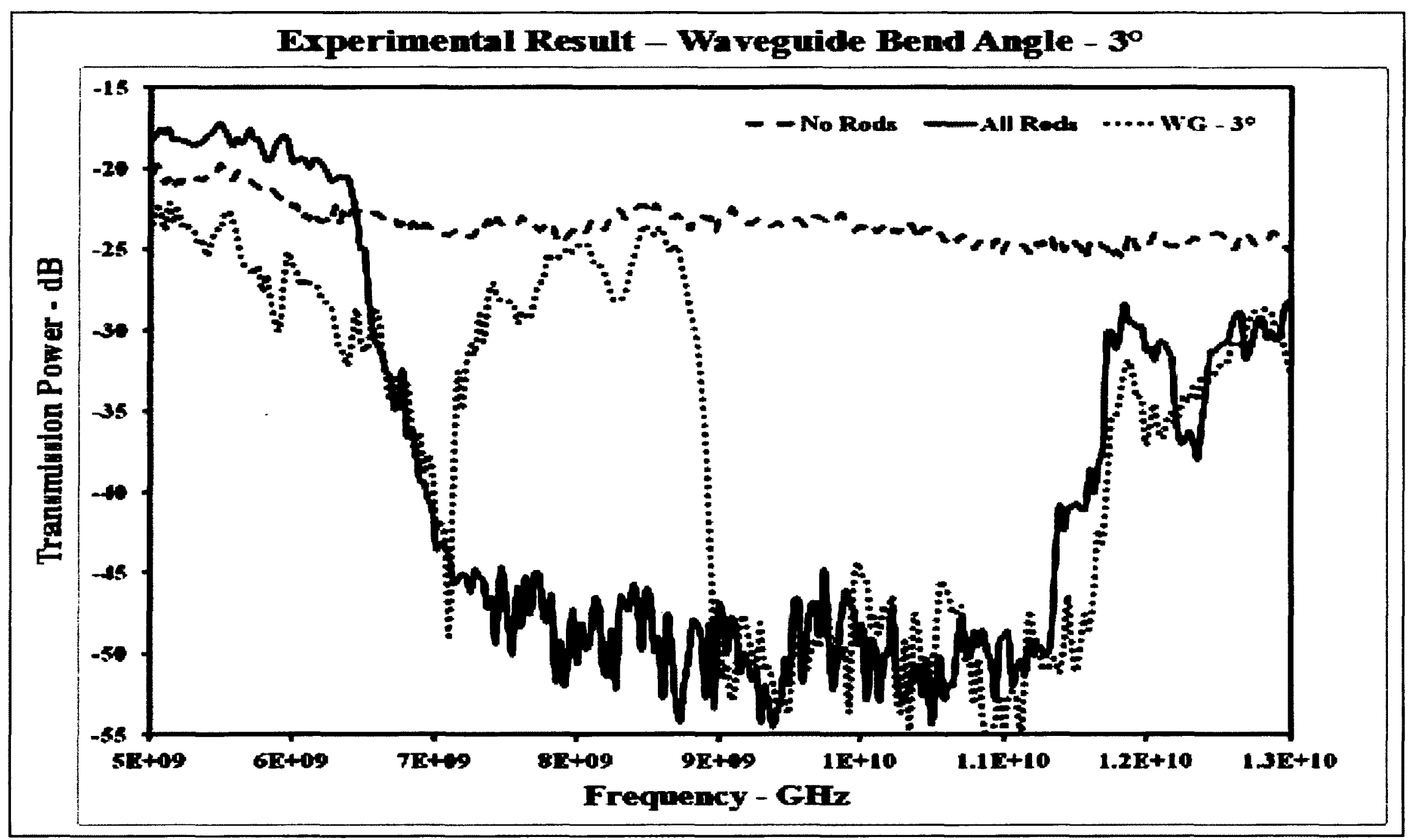

Figure C.3: Transmission spectrum for a linear waveguide with lattice constant, $a=16.9 \mathrm{~mm}$ at bend angle, $\theta=3^{\circ}$ 


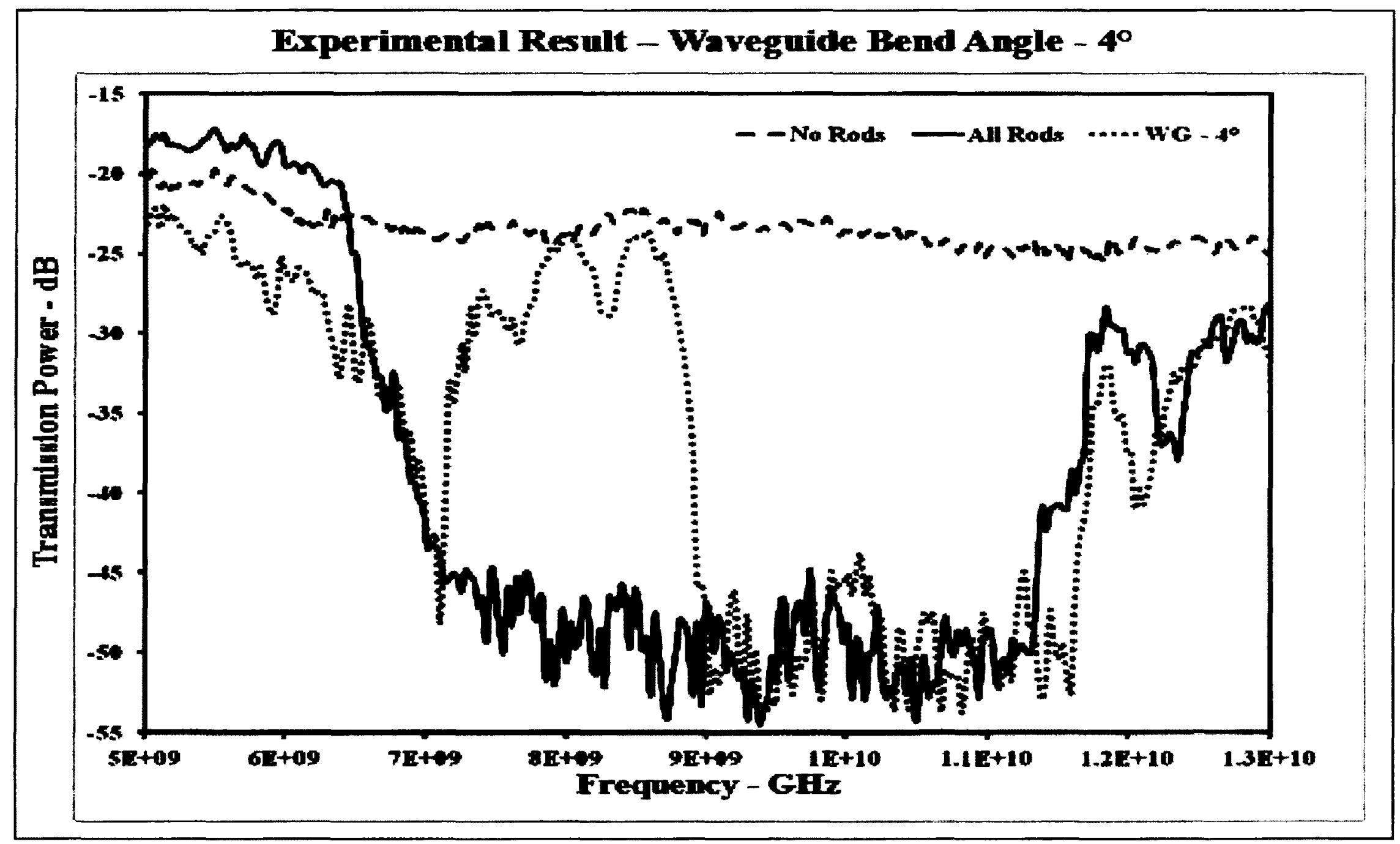

Figure C.4: Transmission spectrum for a linear waveguide with lattice constant, $a=16.9 \mathrm{~mm}$ at bend angle, $\theta=4^{\circ}$ 


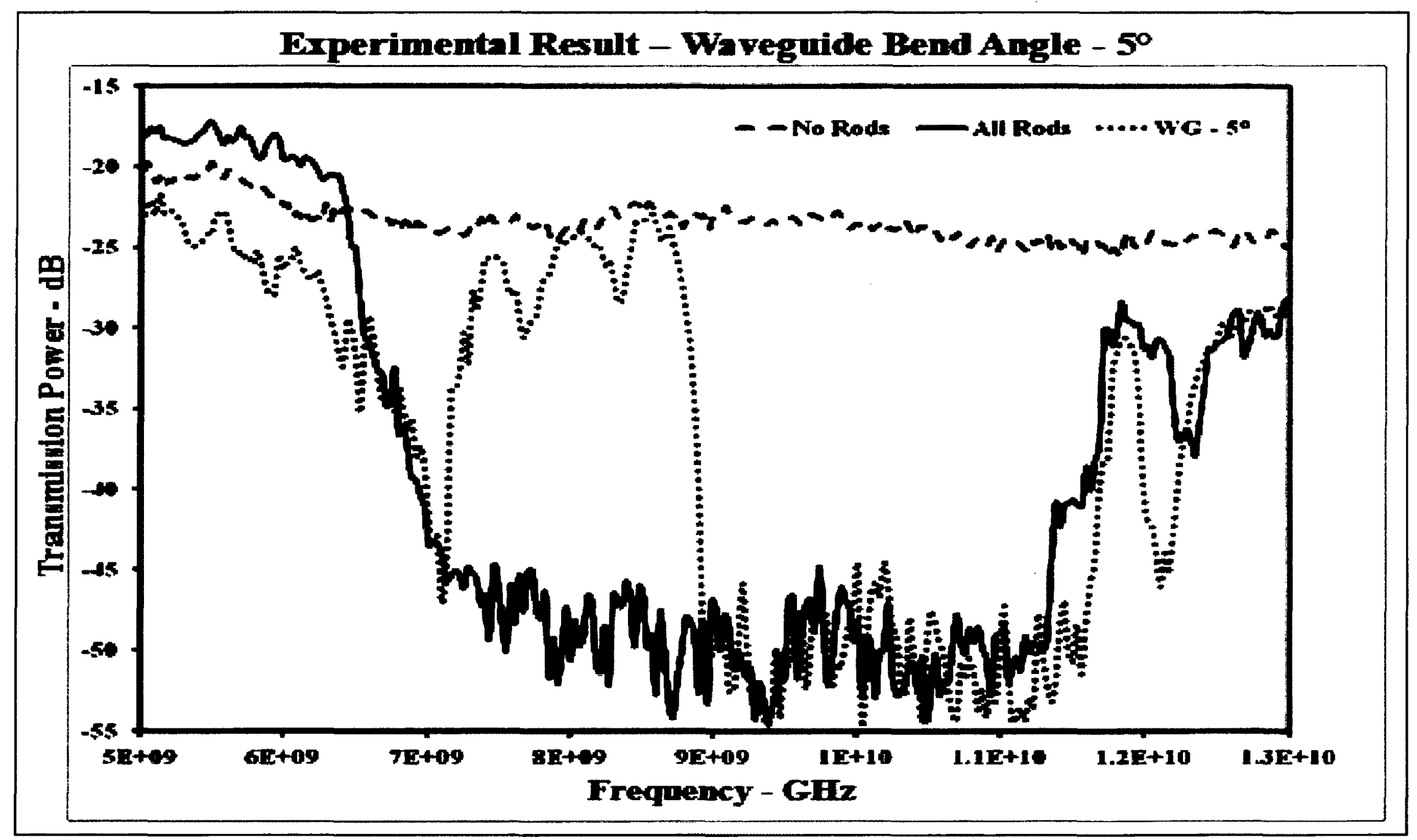

Figure C.5: Transmission spectrum for a linear waveguide with lattice constant, $a=16.9 \mathrm{~mm}$ at bend angle, $\theta=5^{\circ}$ 


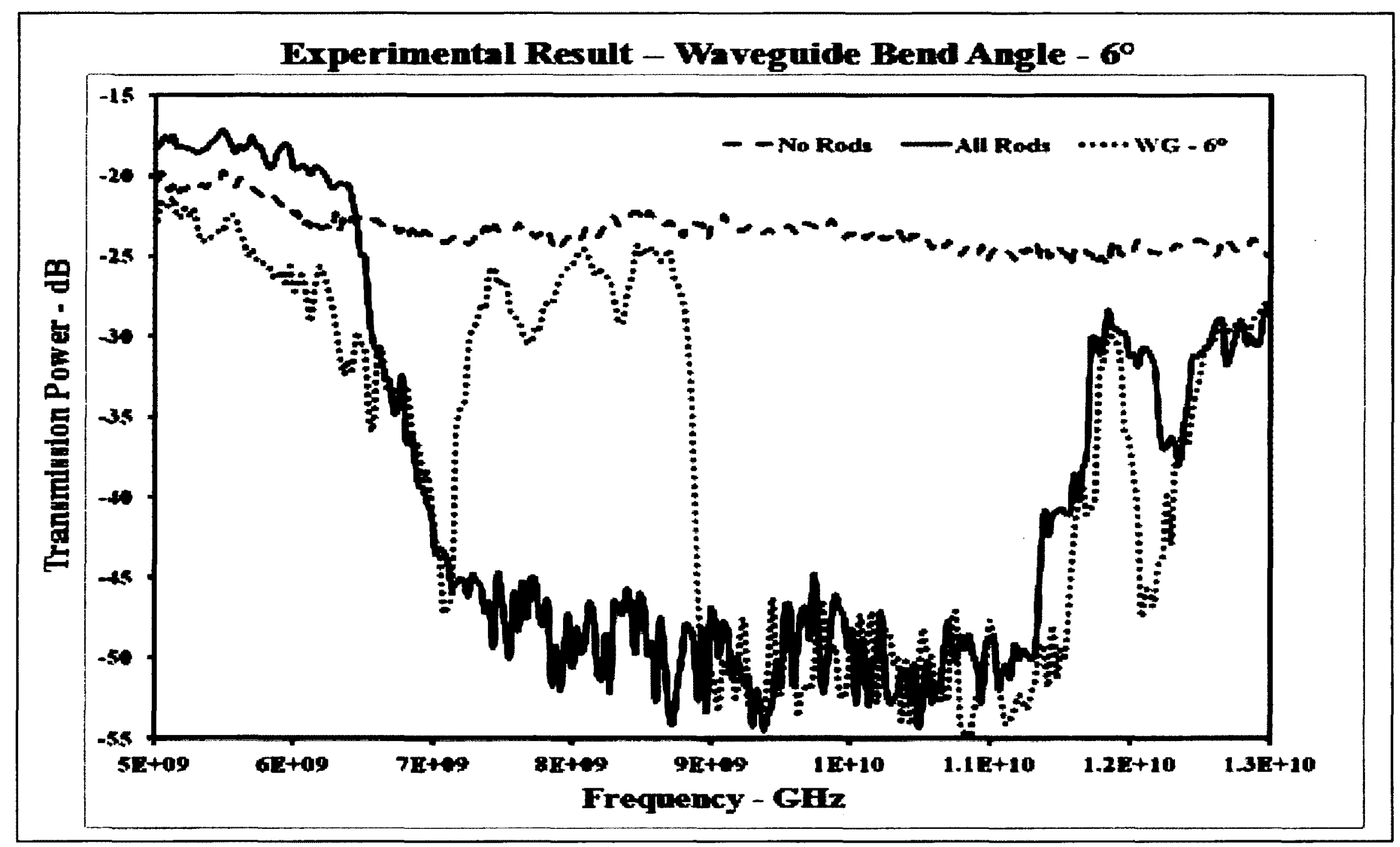

Figure C.6: Transmission spectrum for a linear waveguide with lattice constant, $a=16.9 \mathrm{~mm}$ at bend angle, $\theta=6^{\circ}$ 


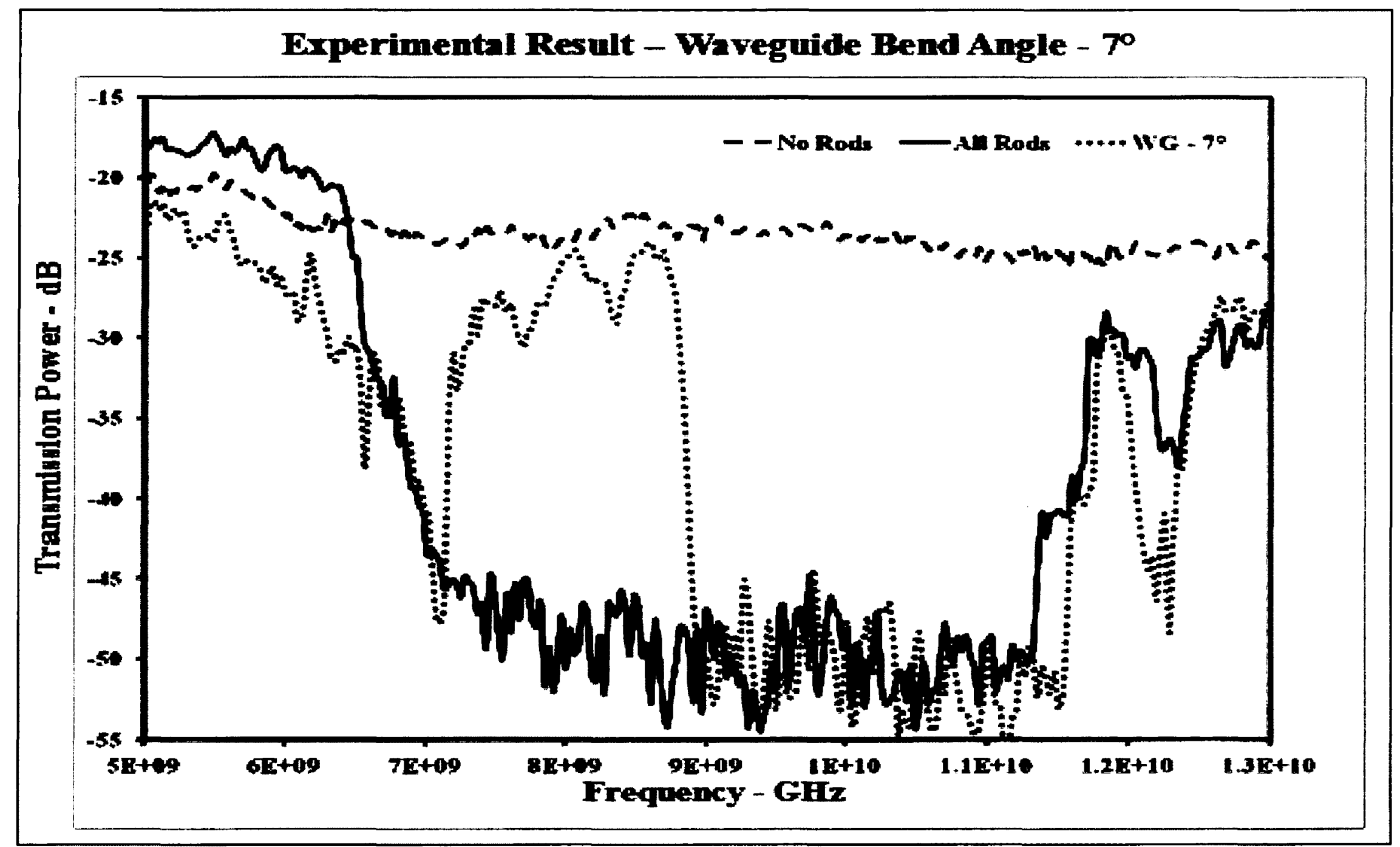

Figure C.7: Transmission spectrum for a linear waveguide with lattice constant, $a=16.9 \mathrm{~mm}$ at bend angle, $\theta=7^{\circ}$ 


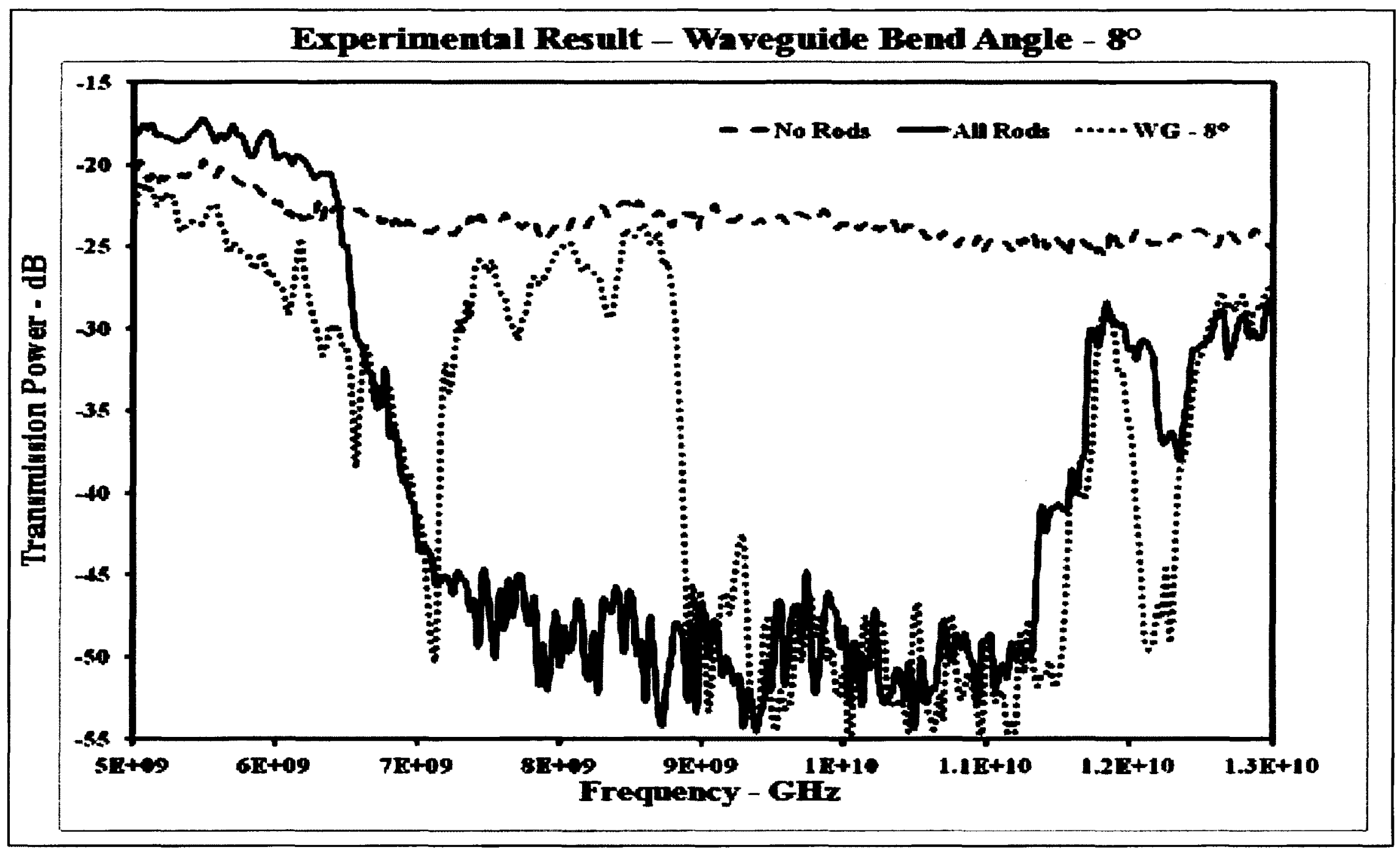

Figure C.8: Transmission spectrum for a linear waveguide with lattice constant, $a=16.9 \mathrm{~mm}$ at bend angle, $\theta=8^{\circ}$

Appendix C

Page 130 


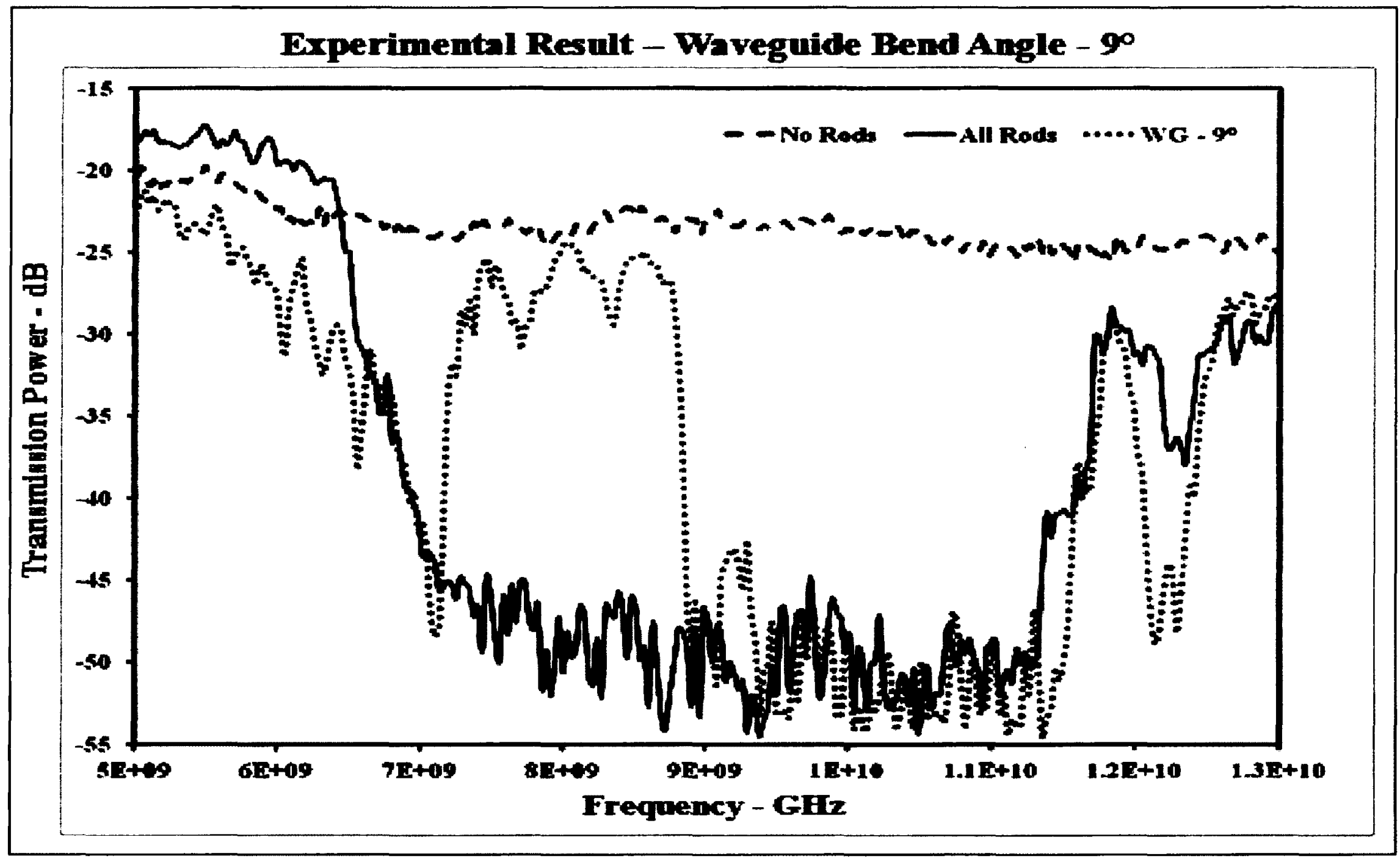

Figure C.9: Transmission spectrum for a linear waveguide with lattice constant, $a=16.9 \mathrm{~mm}$ at bend angle, $\theta=9^{\circ}$ 


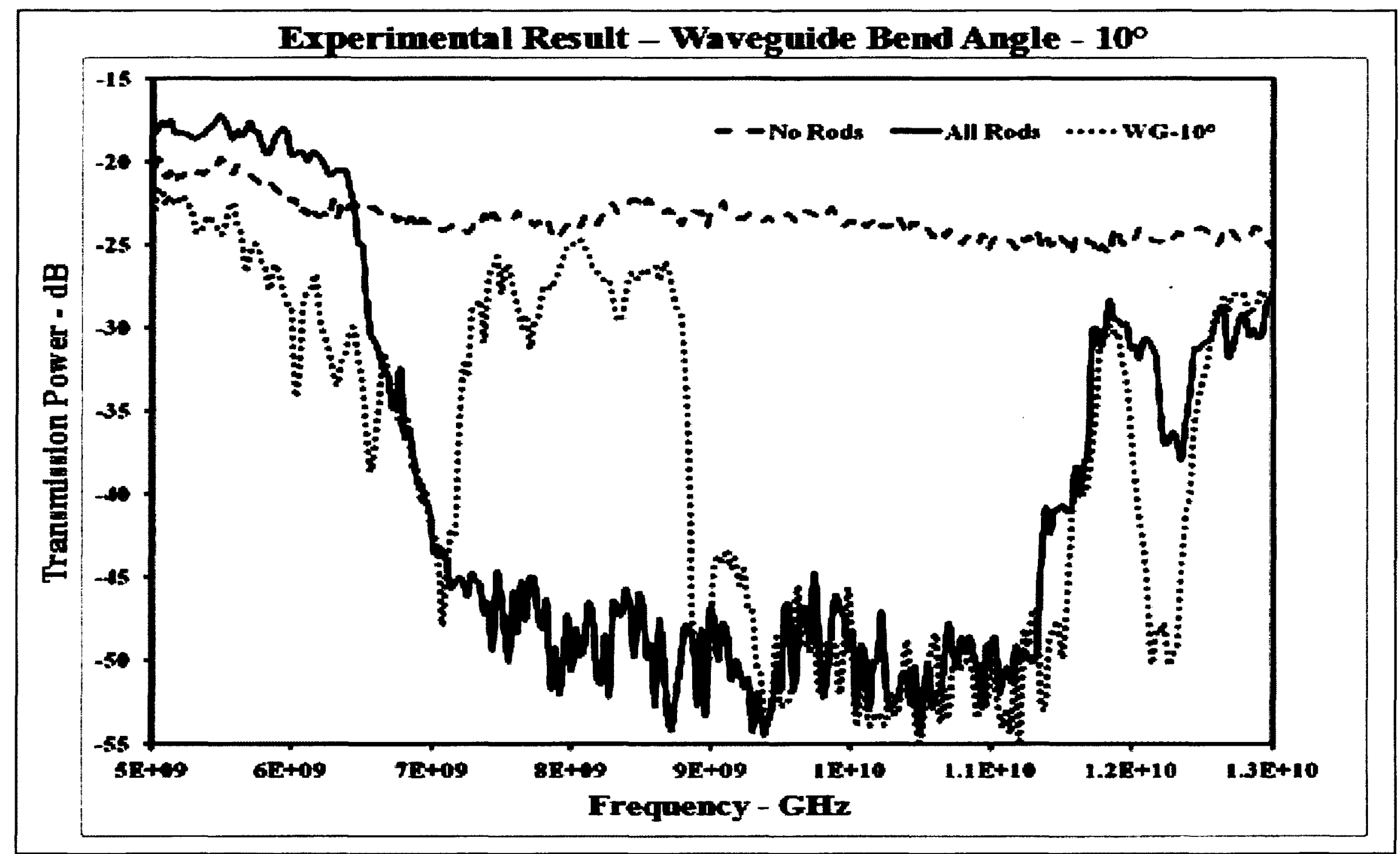

Figure C.10: Transmission spectrum for a linear waveguide with lattice constant, $a=16.9 \mathrm{~mm}$ at bend angle, $\theta=10^{\circ}$ 


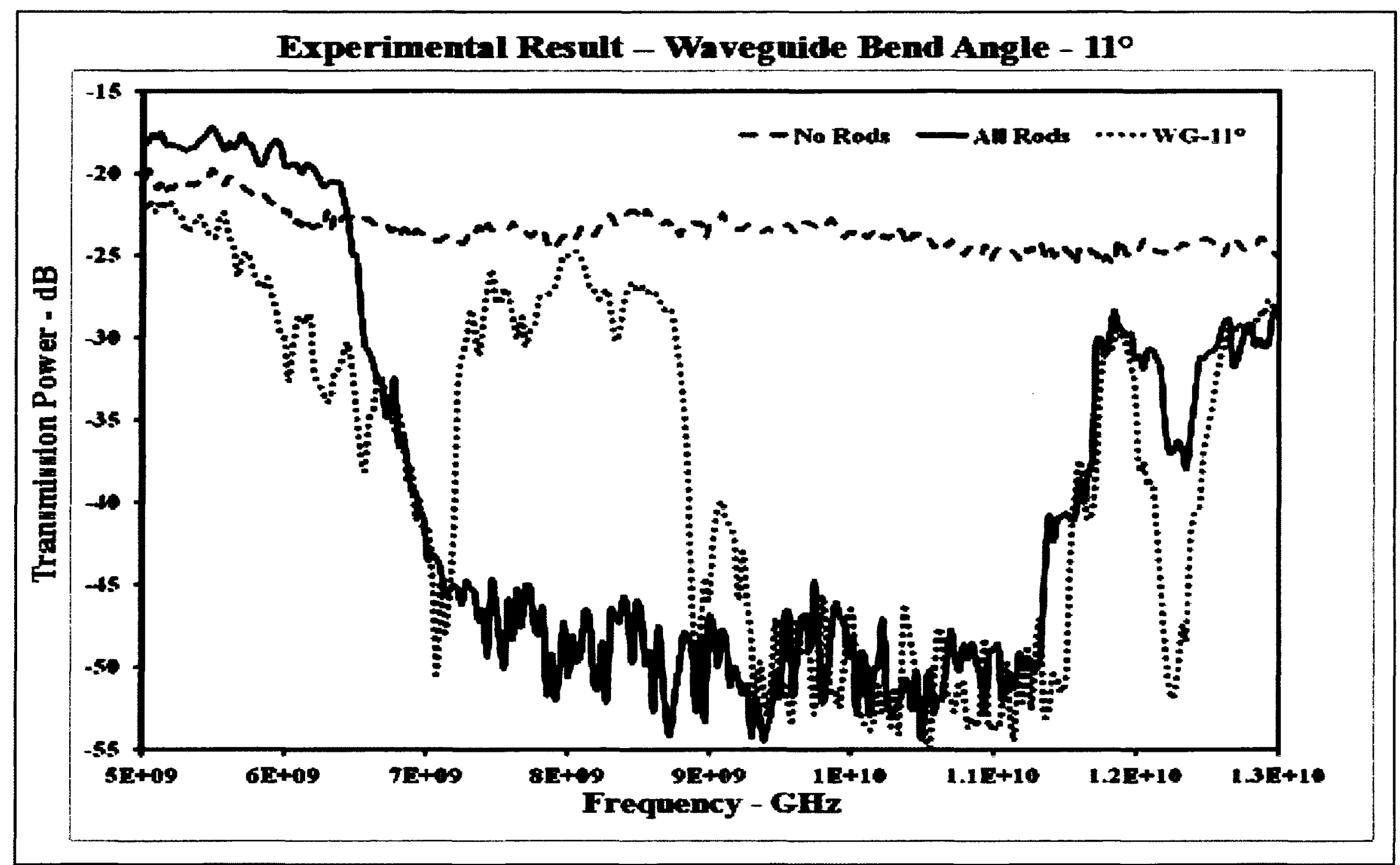

Figure C.11: Transmission spectrum for a linear waveguide with lattice constant, $a=16.9 \mathrm{~mm}$ at bend angle, $\theta=11^{\circ}$ 


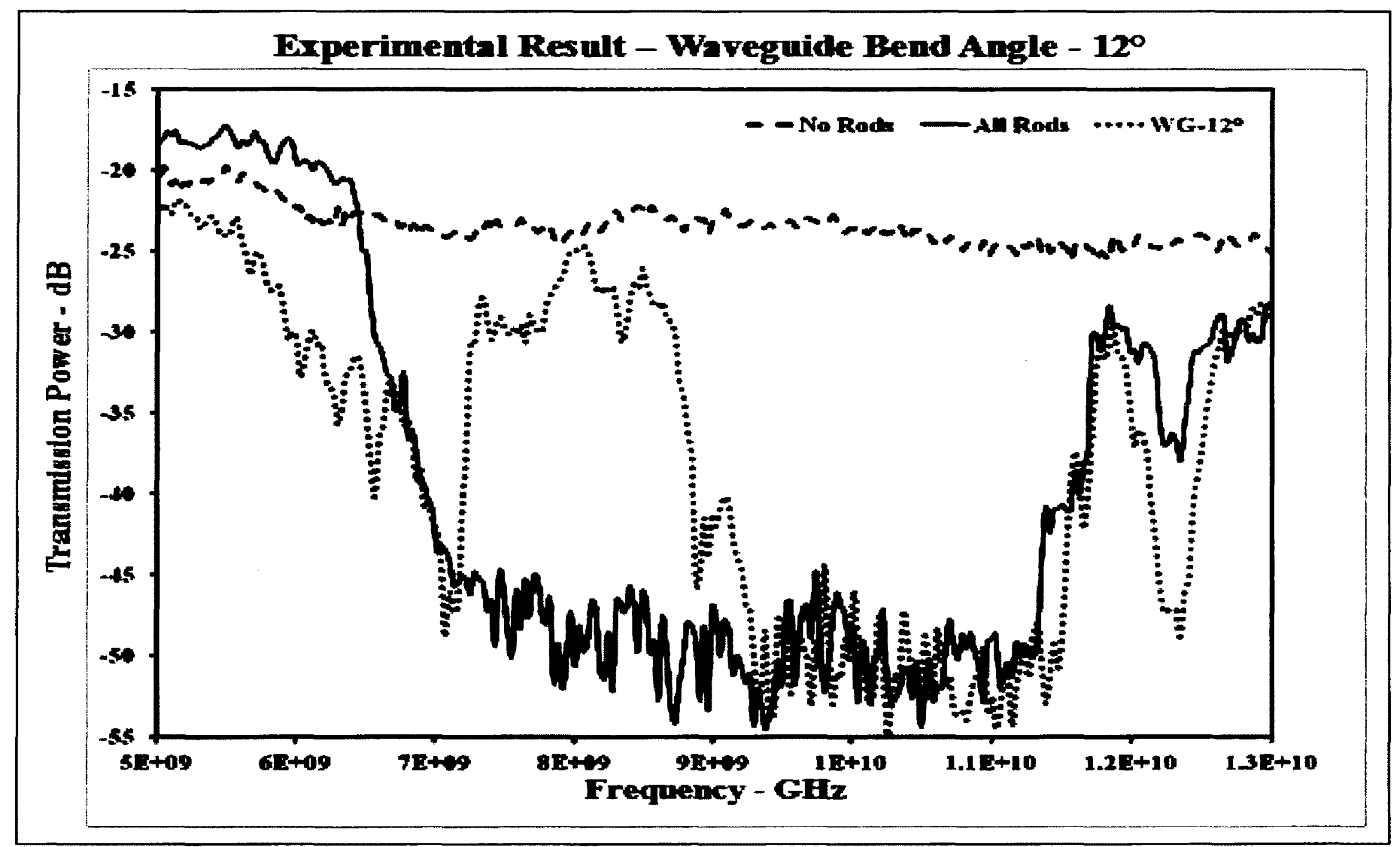

Figure C.12: Transmission spectrum for a linear waveguide with lattice constant, $a=16.9 \mathrm{~mm}$ at bend angle, $\theta=12^{\circ}$ 


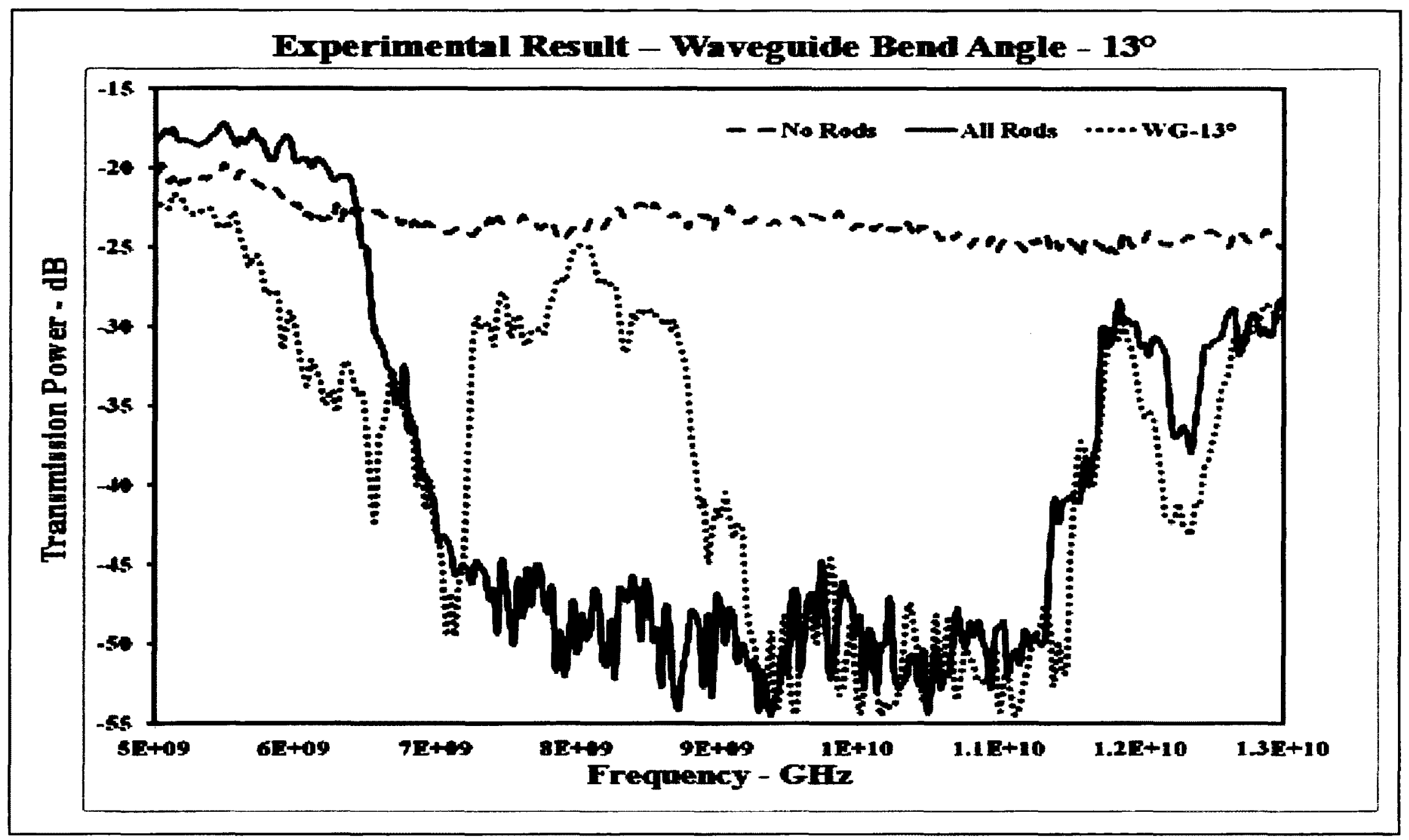

Figure C.13: Transmission spectrum for a linear waveguide with lattice constant, $a=16.9 \mathrm{~mm}$ at bend angle, $\theta=13^{\circ}$ 


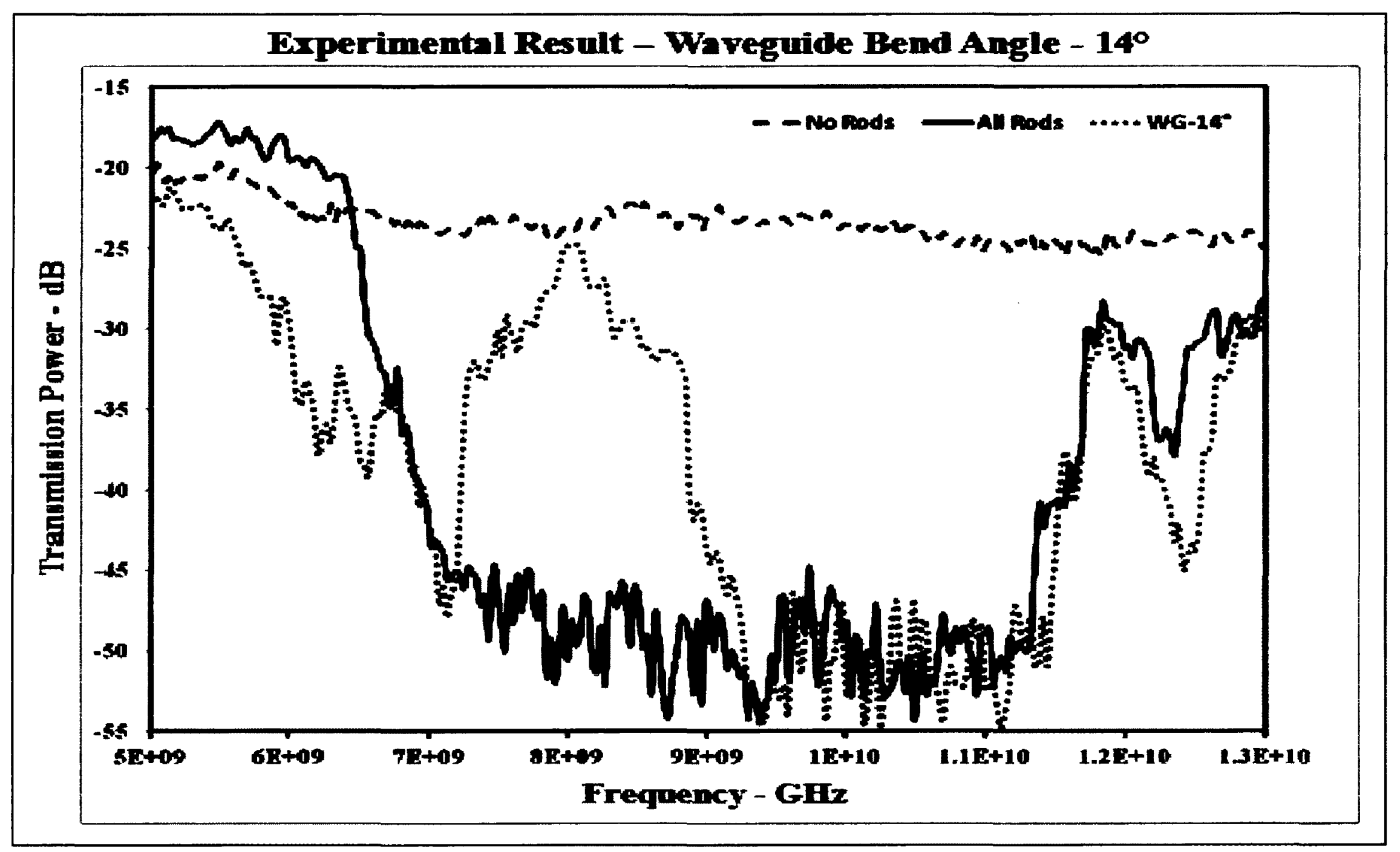

Figure C.14: Transmission spectrum for a linear waveguide with lattice constant, $a=16.9 \mathrm{~mm}$ at bend angle, $\theta=14^{\circ}$ 


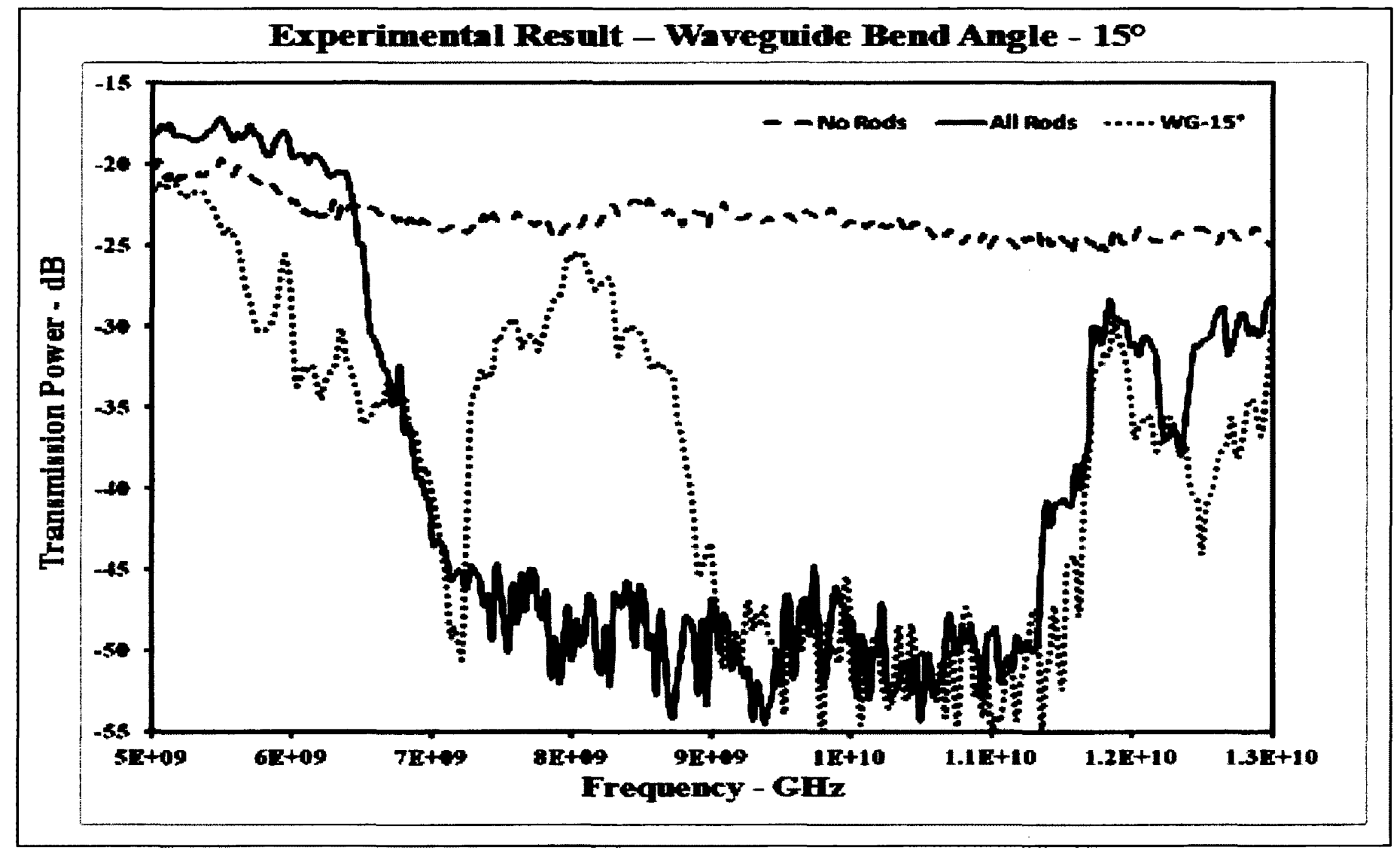

Figure C.15: Transmission spectrum for a linear waveguide with lattice constant, $a=16.9 \mathrm{~mm}$ at bend angle, $\theta=15^{\circ}$ 


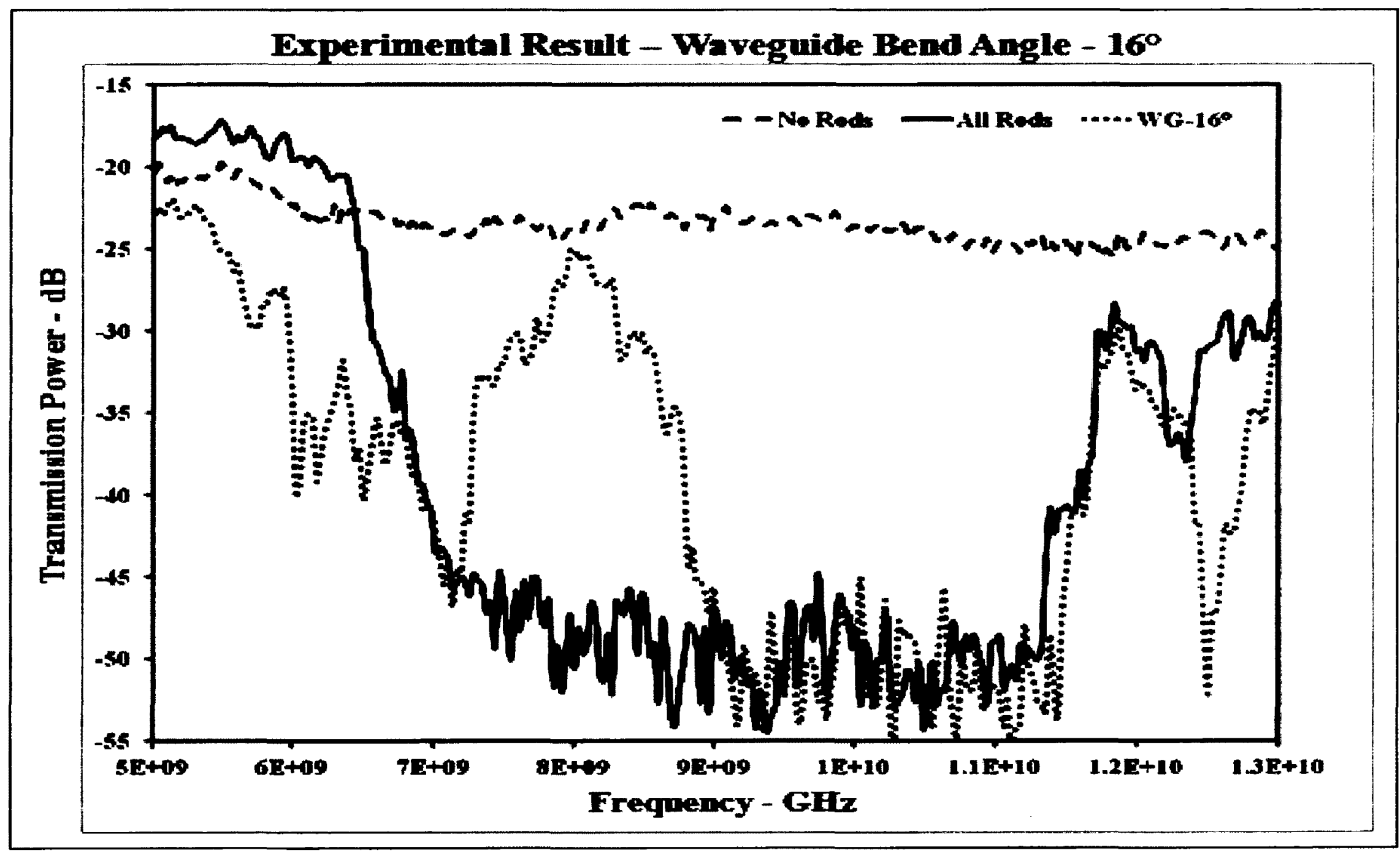

Figure C.16: Transmission spectrum for a linear waveguide with lattice constant, $a=16.9 \mathrm{~mm}$ at bend angle, $\theta=16^{\circ}$

Appendix C

Page 138 


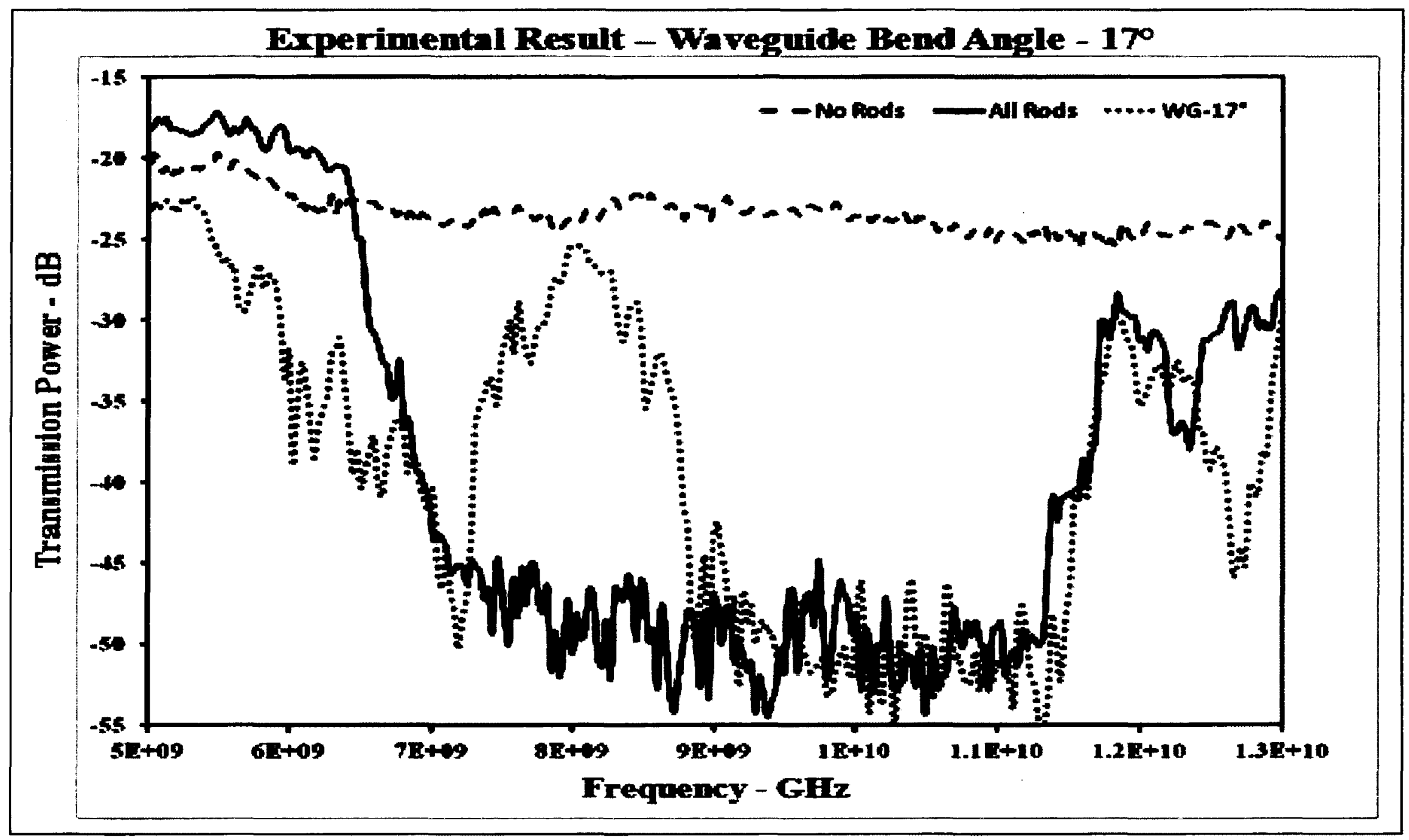

Figure C.17: Transmission spectrum for a linear waveguide with lattice constant, $a=16.9 \mathrm{~mm}$ at bend angle, $\theta=17^{\circ}$ 


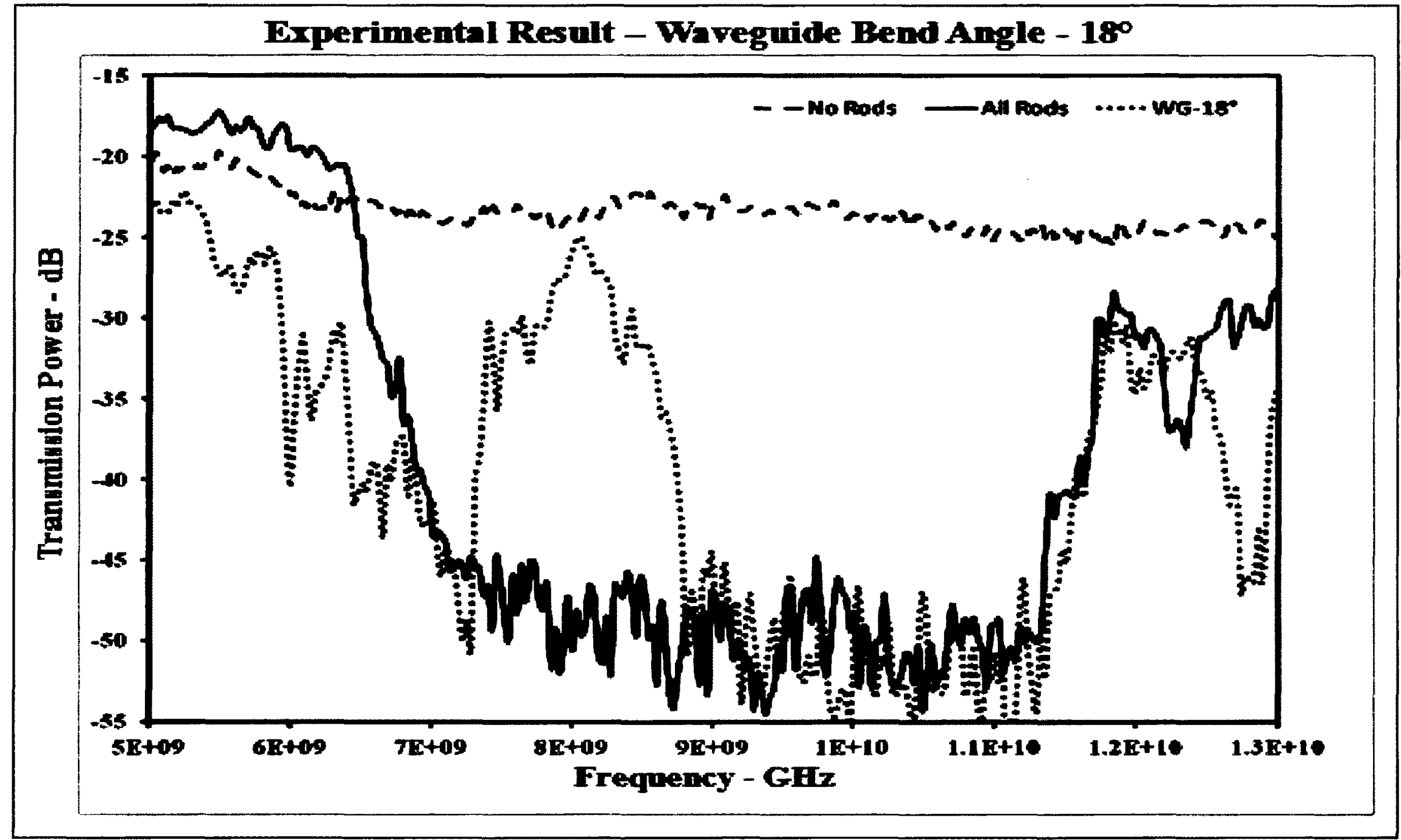

Figure C.18: Transmission spectrum for a linear waveguide with lattice constant, $a=16.9 \mathrm{~mm}$ at bend angle, $\theta=18^{\circ}$ 


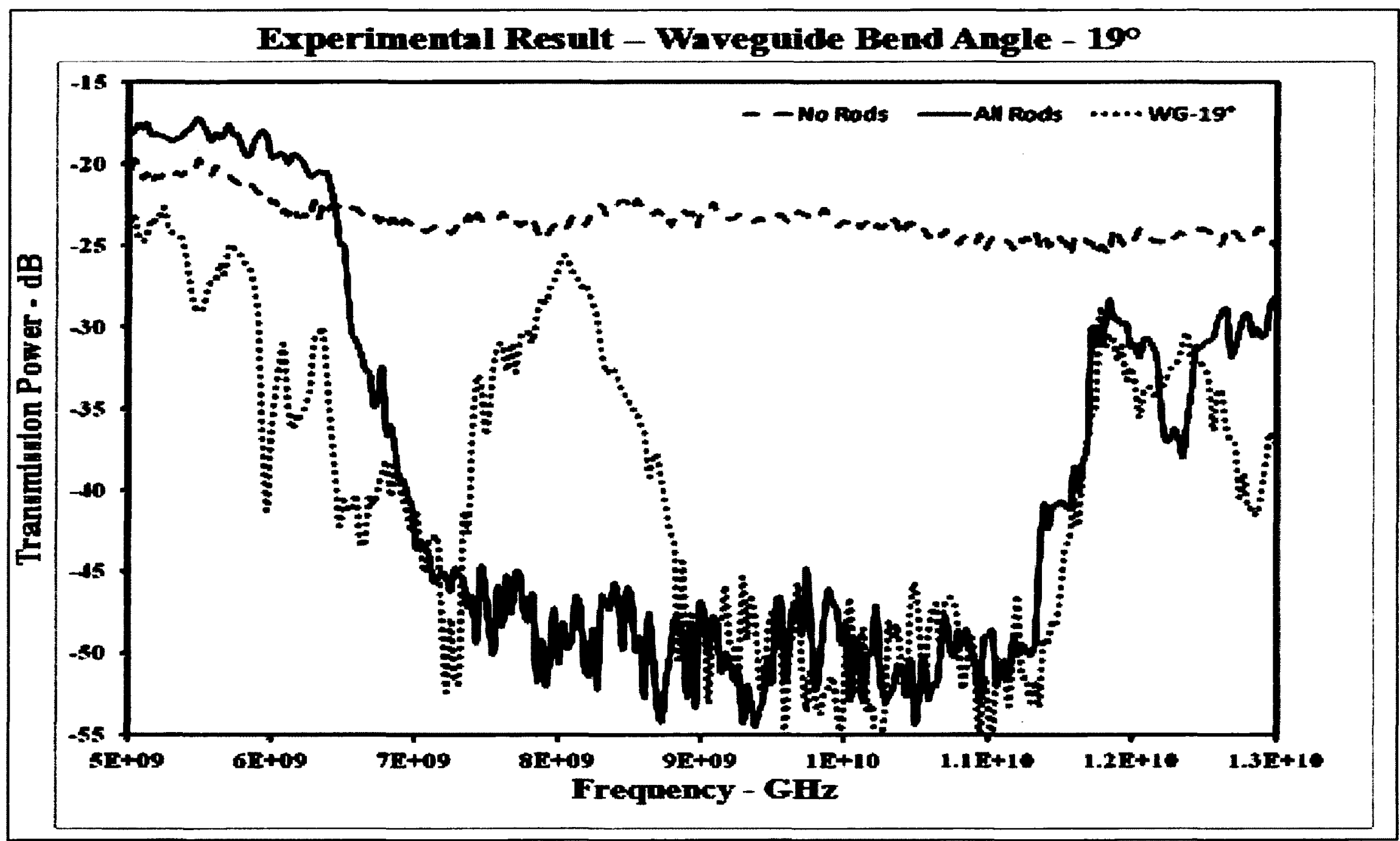

Figure C.19: Transmission spectrum for a linear waveguide with lattice constant, $a=16.9 \mathrm{~mm}$ at bend angle, $\theta=19^{\circ}$ 


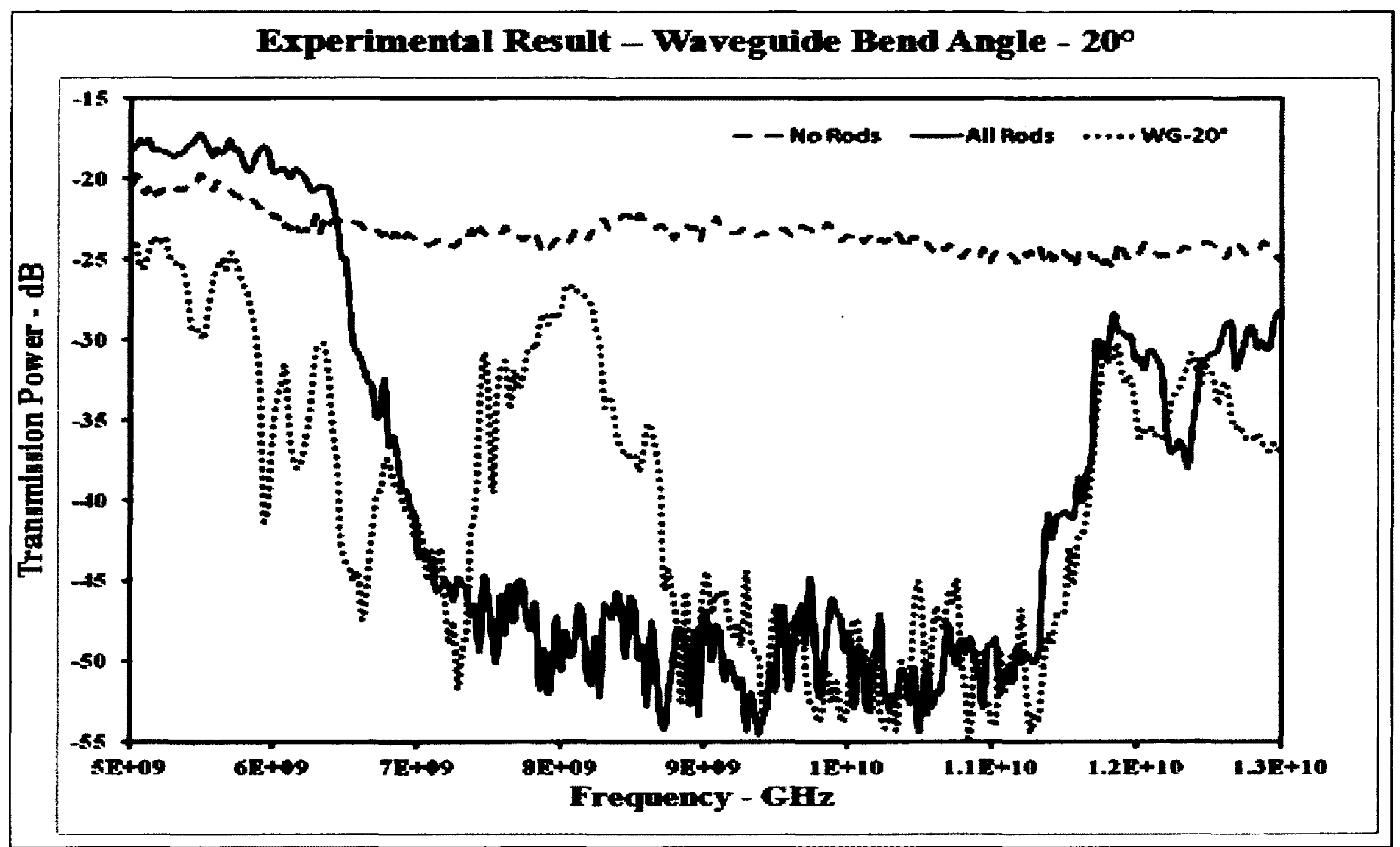

Figure C.20: Transmission spectrum for a linear waveguide with lattice constant, $a=16.9 \mathrm{~mm}$ at bend angle, $\theta=20^{\circ}$ 


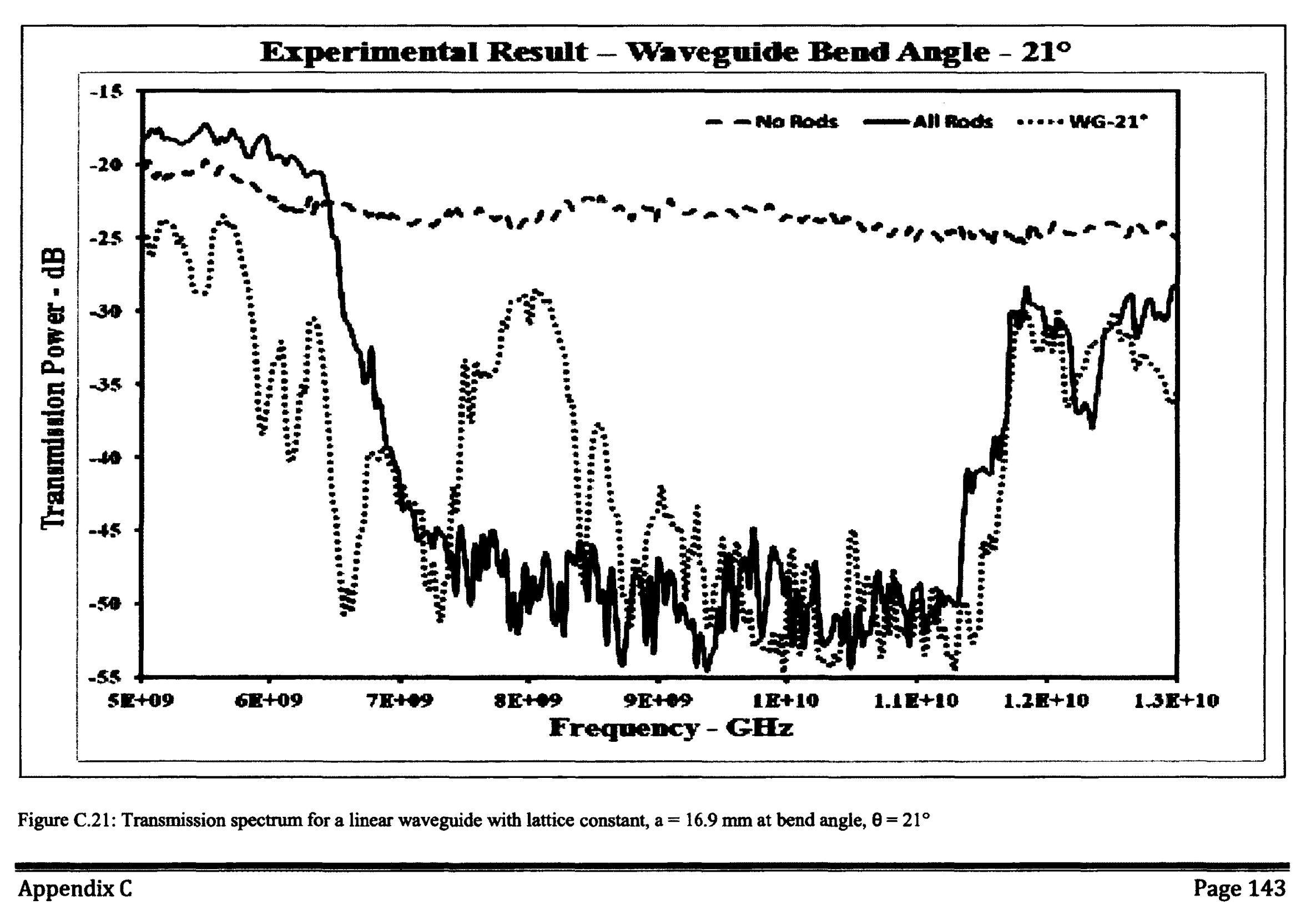




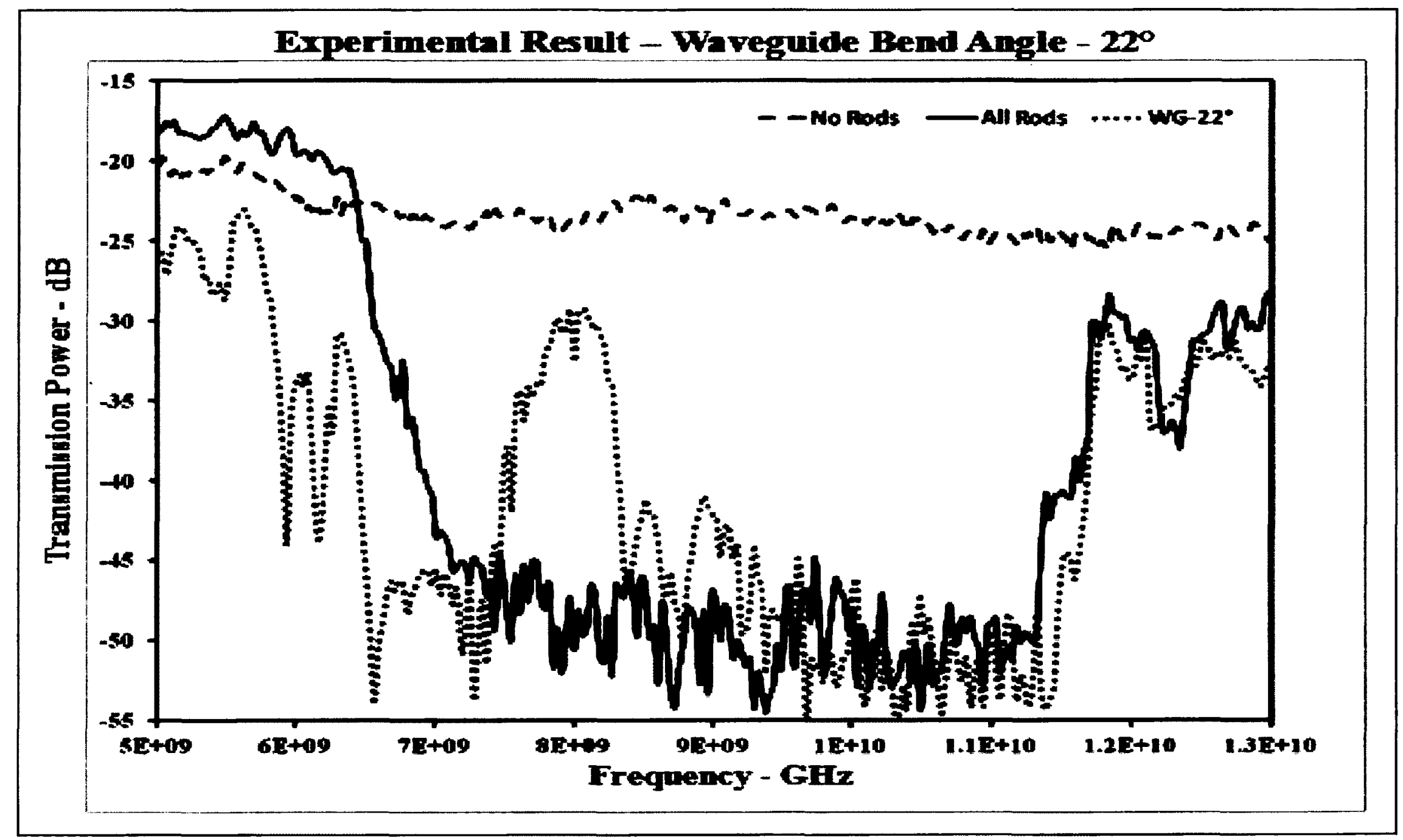

Figure C.22: Transmission spectrum for a linear waveguide with lattice constant, $a=16.9 \mathrm{~mm}$ at bend angle, $\theta=22^{\circ}$ 


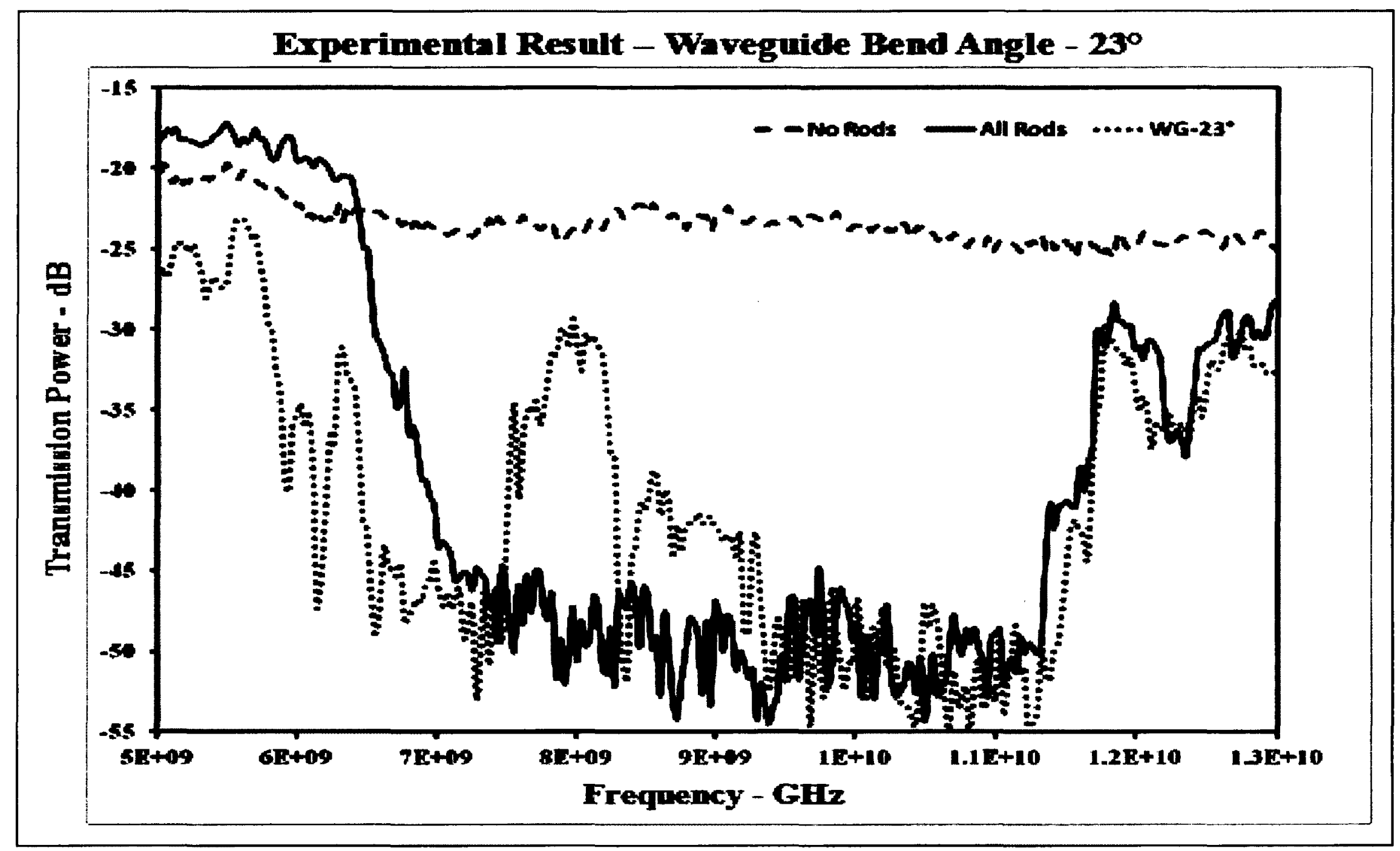

Figure C.23: Transmission spectrum for a linear waveguide with lattice constant, $a=16.9 \mathrm{~mm}$ at bend angle, $\theta=23^{\circ}$ 


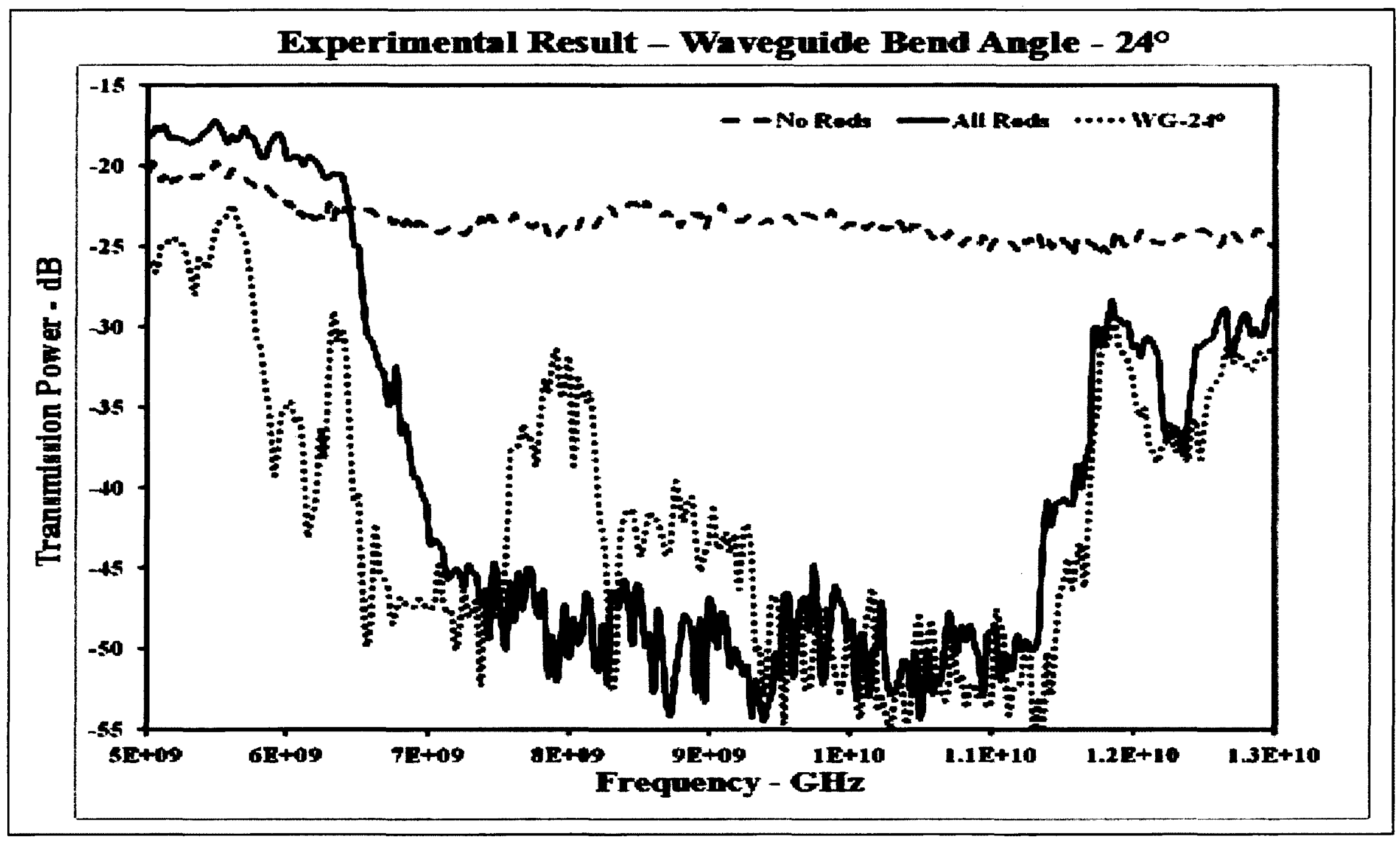

Figure C.24: Transmission spectrum for a linear waveguide with lattice constant, $a=16.9 \mathrm{~mm}$ at bend angle, $\theta=24^{\circ}$ 


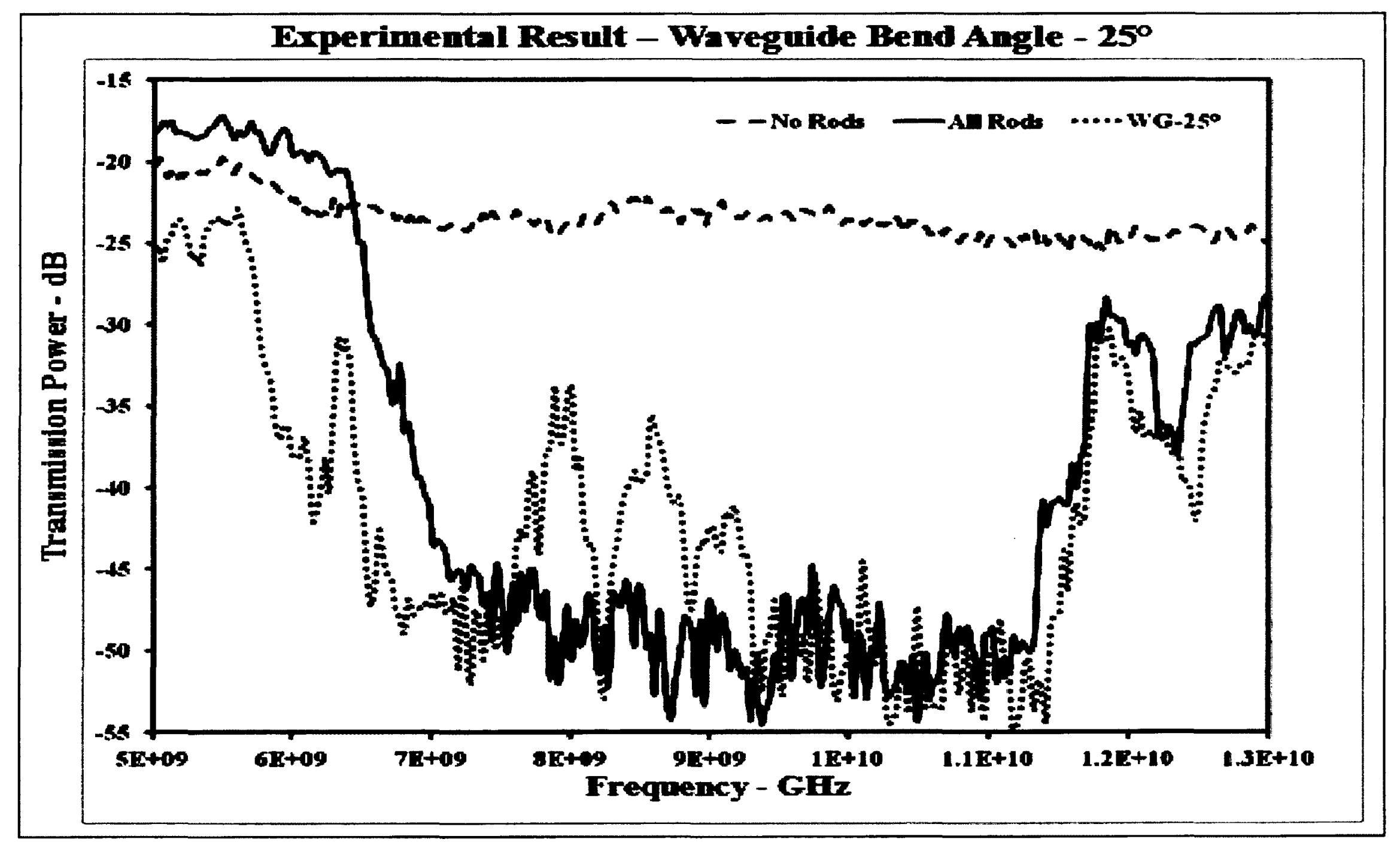

Figure C.25: Transmission spectrum for a linear waveguide with lattice constant, $a=16.9 \mathrm{~mm}$ at bend angle, $\theta=25^{\circ}$ 


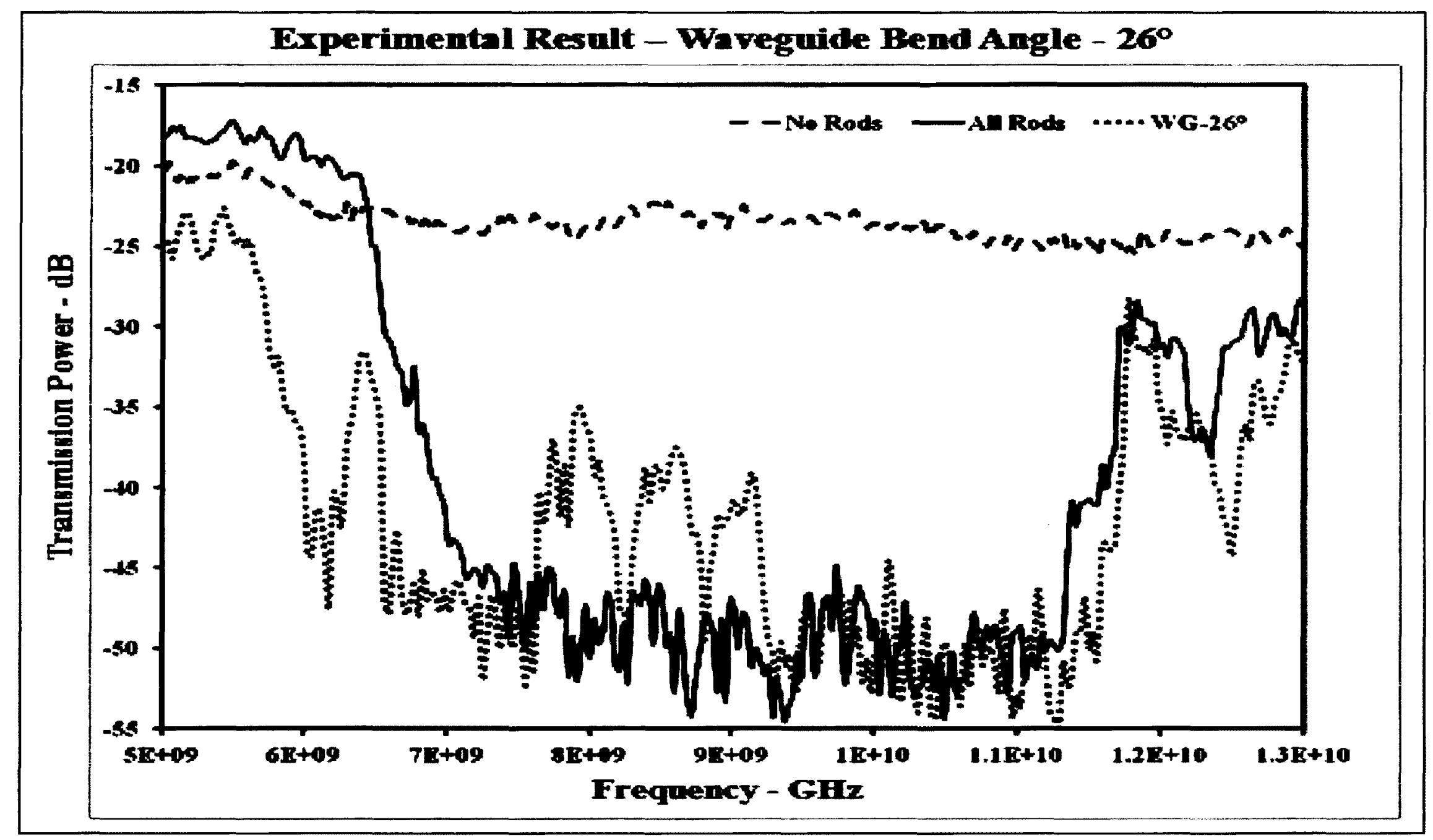

Figure C.26: Transmission spectrum for a linear waveguide with lattice constant, $a=16.9 \mathrm{~mm}$ at bend angle, $\theta=26^{\circ}$ 


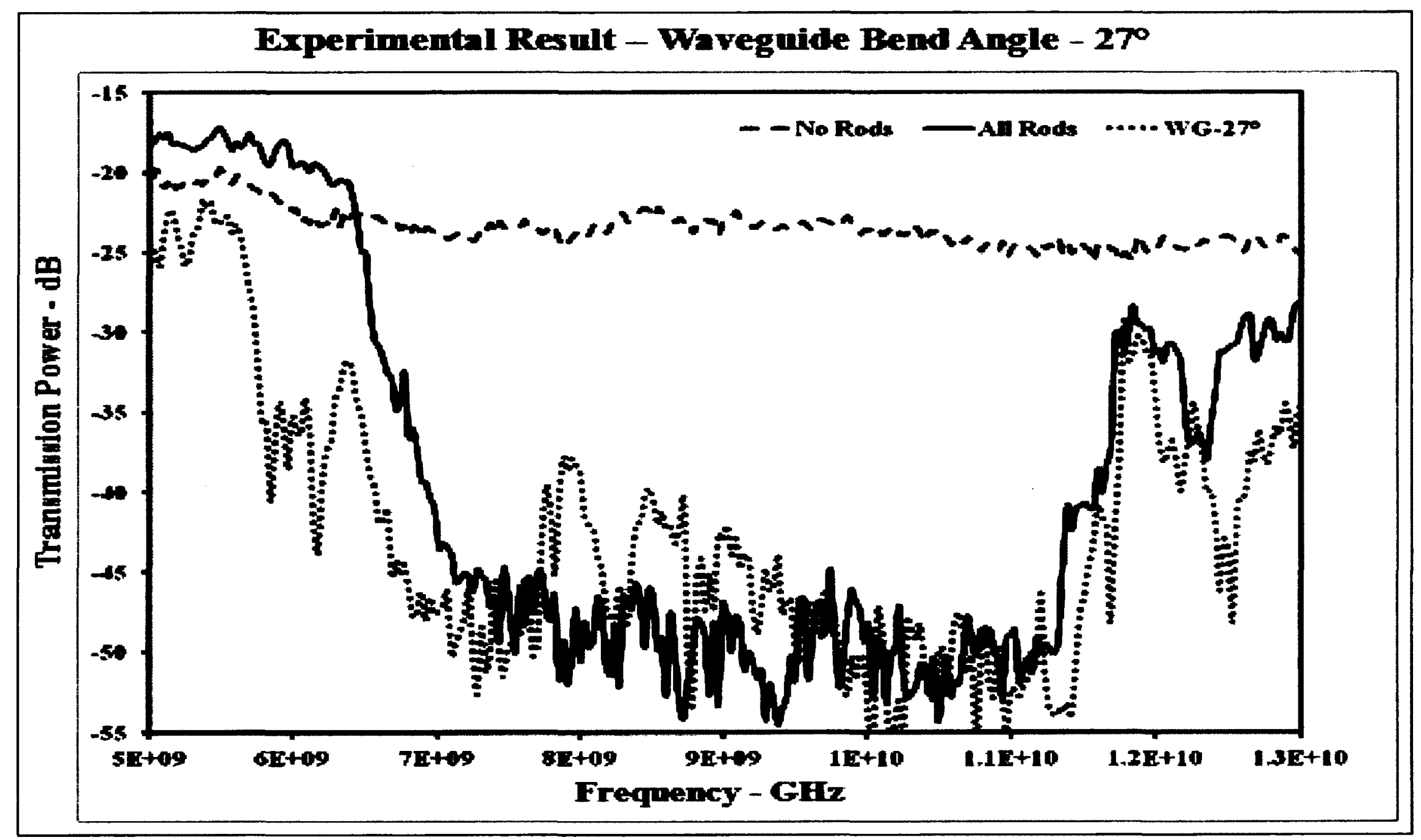

Figure C.27: Transmission spectrum for a linear waveguide with lattice constant, $a=16.9 \mathrm{~mm}$ at bend angle, $\theta=27^{\circ}$ 


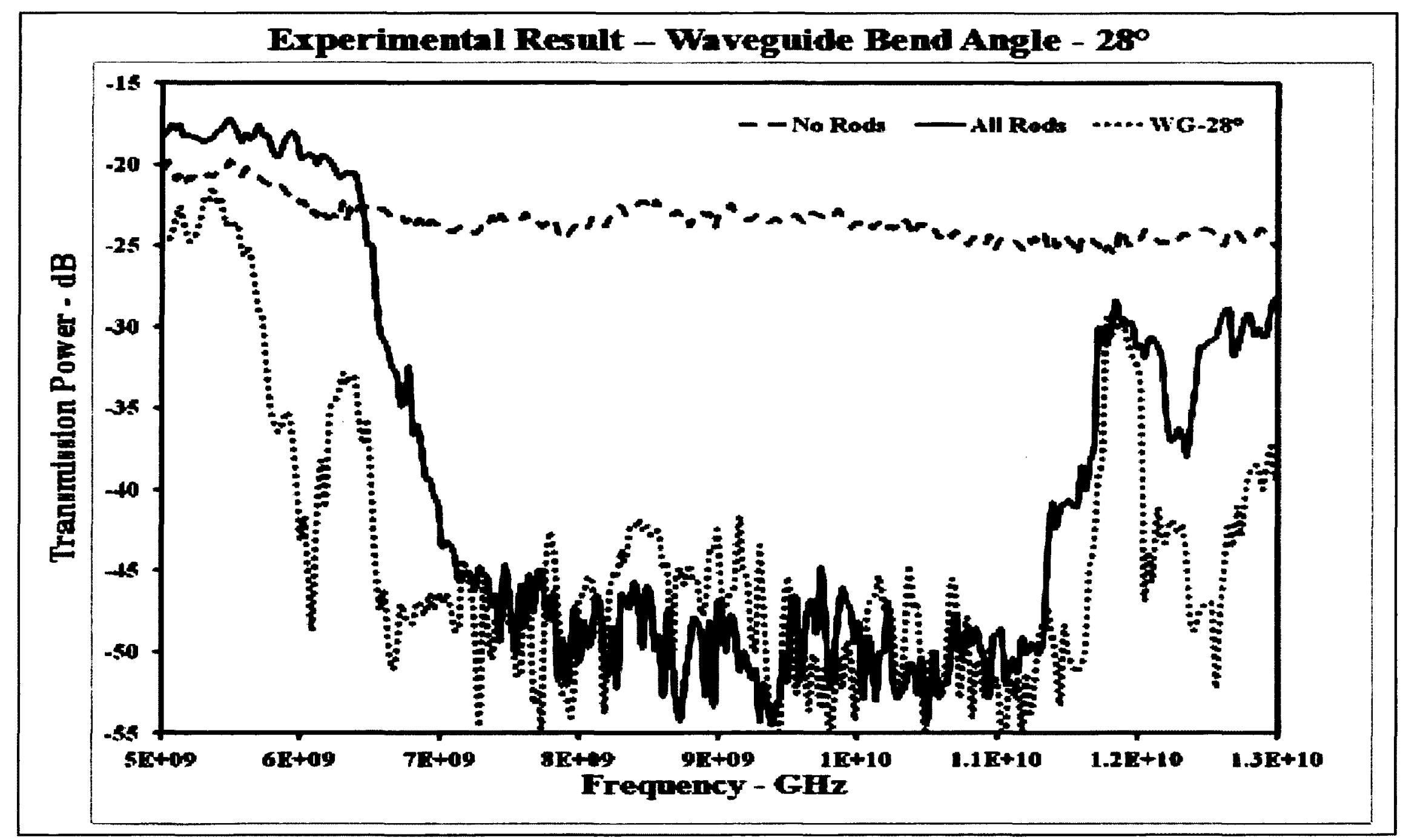

Figure C.28: Transmission spectrum for a linear waveguide with lattice constant, $a=16.9 \mathrm{~mm}$ at bend angle, $\theta=28^{\circ}$ 


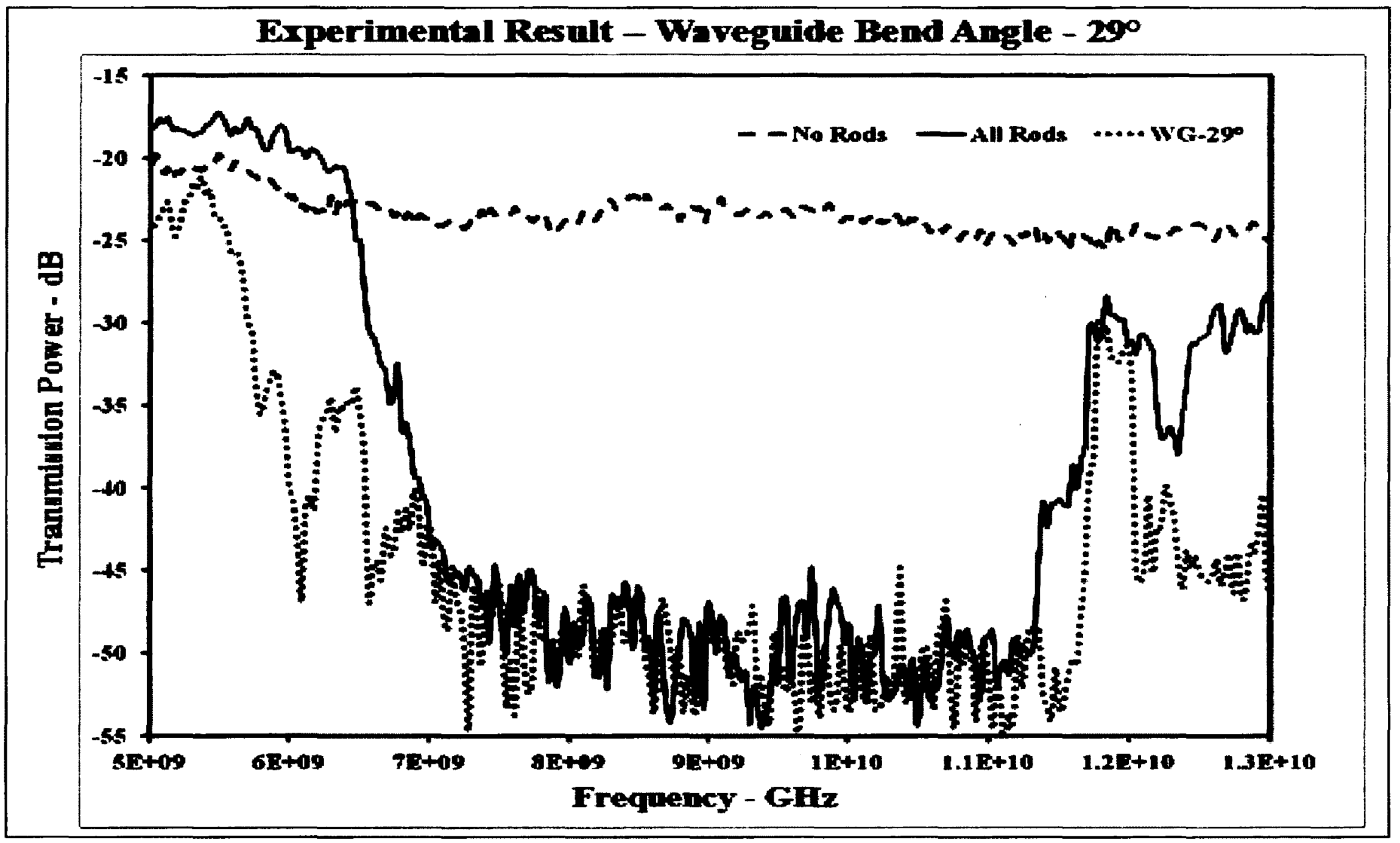

Figure C.29: Transmission spectrum for a linear waveguide with lattice constant, $a=16.9 \mathrm{~mm}$ at bend angle, $\theta=29^{\circ}$ 


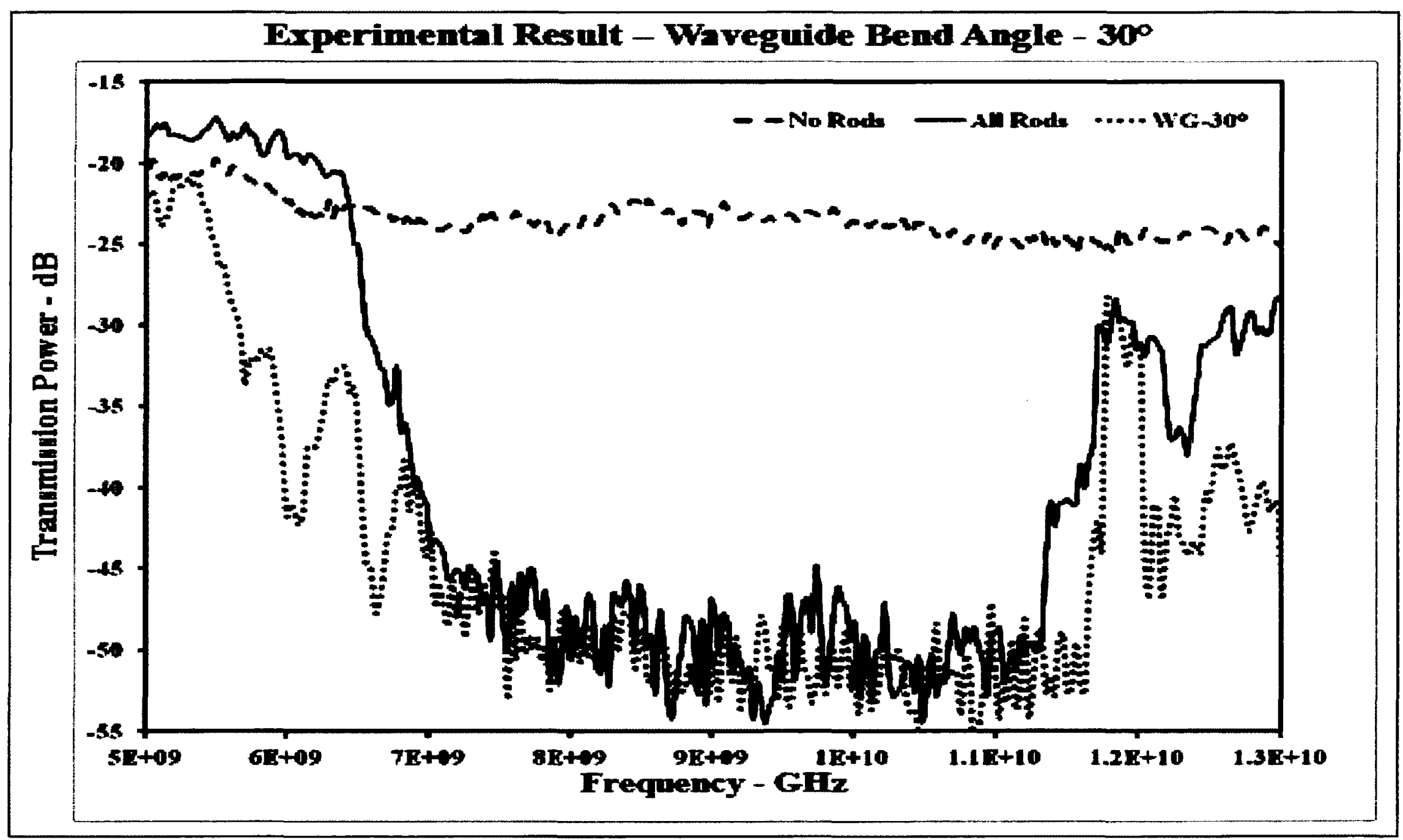

Figure C.30: Transmission spectrum for a linear waveguide with lattice constant, $a=16.9 \mathrm{~mm}$ at bend angle, $\theta=30^{\circ}$ 


\section{Appendix D:}

Relative Transmission Loss Versus Waveguide Bend Angle, $\theta$ for a Set of

\section{Frequencies}

( $f=8.57 \mathrm{GHz}$ to $8.81 \mathrm{GHz})$ 


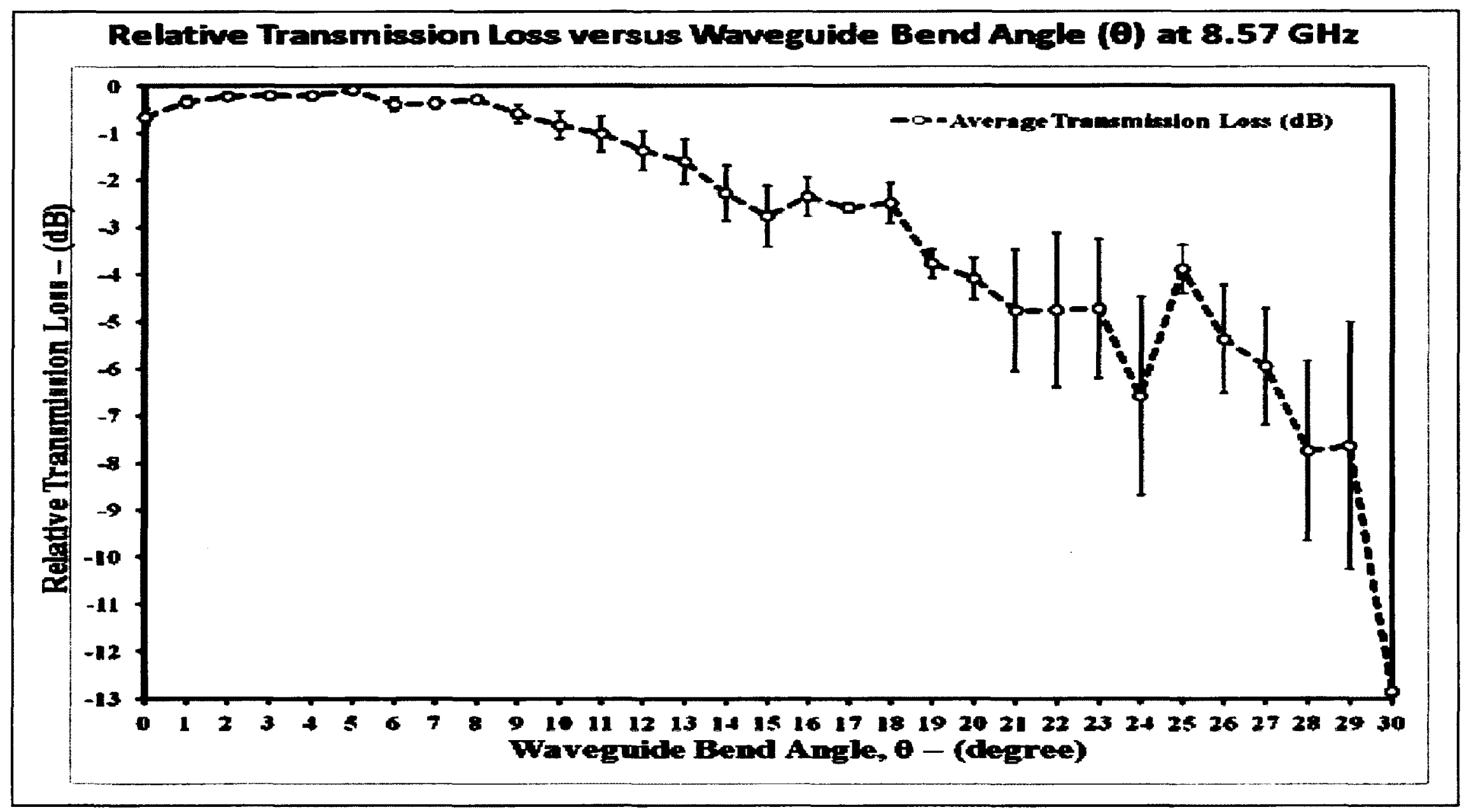

Figure D.1: Plot drawn at a particular frequency point, $\mathrm{f}=8.57 \mathrm{GHz}$ with different waveguide bend angle, $\theta$ versus relative transmission loss in microwave regime. 


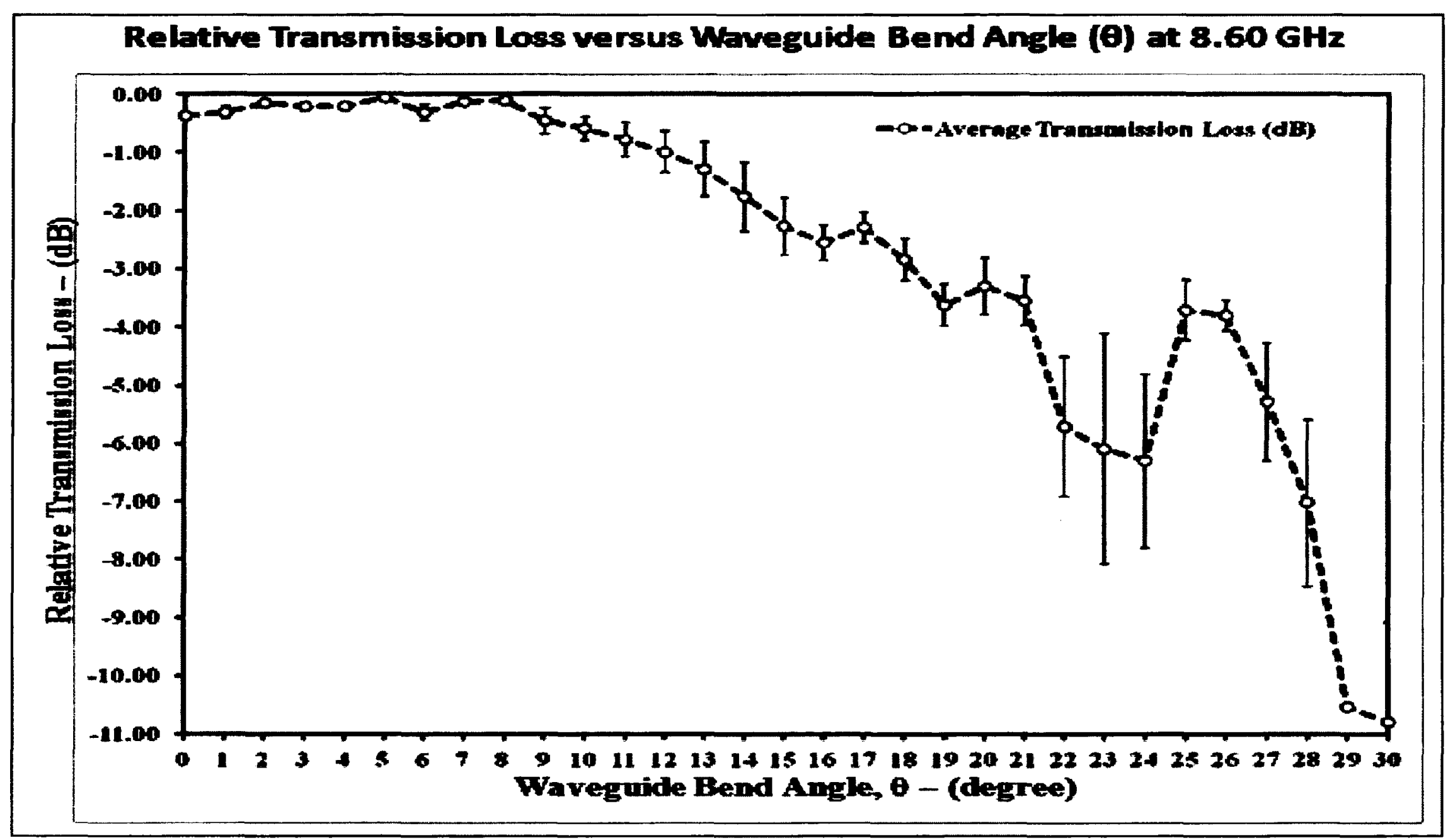

Figure D.2: Plot drawn at a particular frequency point, $\mathrm{f}=8.60 \mathrm{GHz}$ with different waveguide bend angle, $\theta$ versus relative transmission loss in microwave regime. 


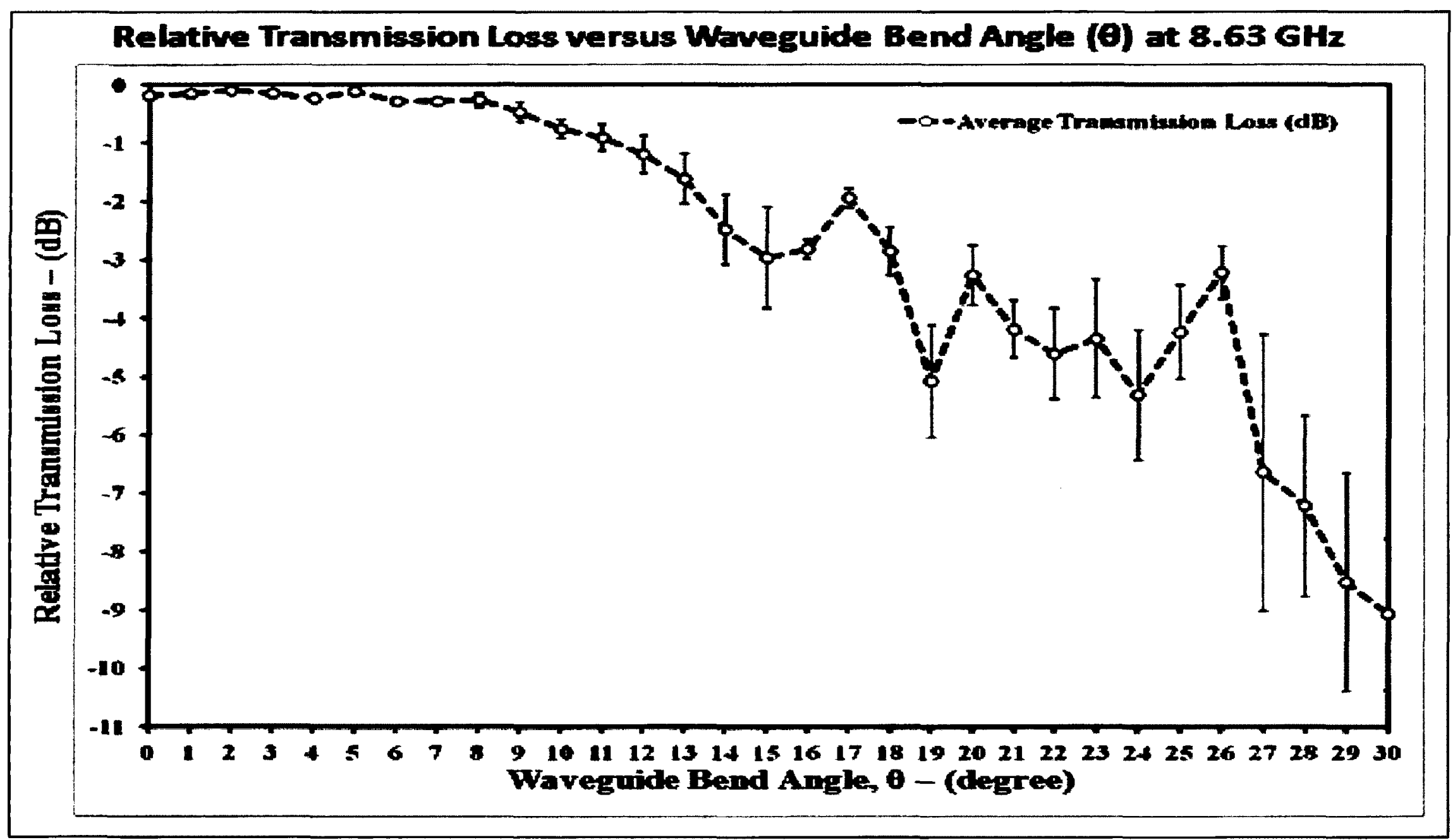

Figure D.3: Plot drawn at a particular frequency point, $f=8.63 \mathrm{GHz}$ with different waveguide bend angle, $\theta$ versus relative transmission loss in microwave regime. 


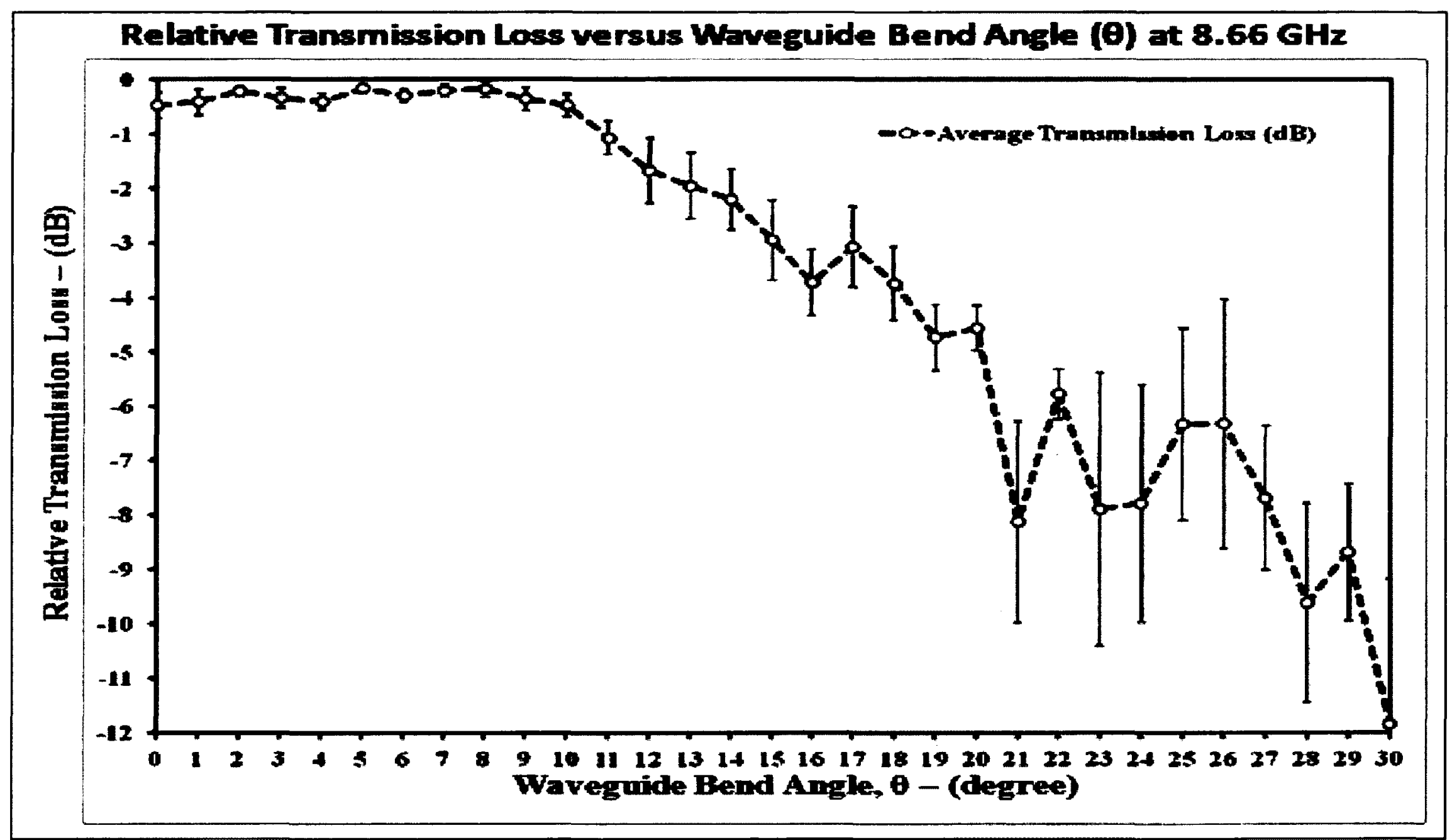

Figure D.4: Plot drawn at a particular frequency point, $\mathrm{f}=8.66 \mathrm{GHz}$ with different waveguide bend angle, $\theta$ versus relative transmission loss in microwave regime. 


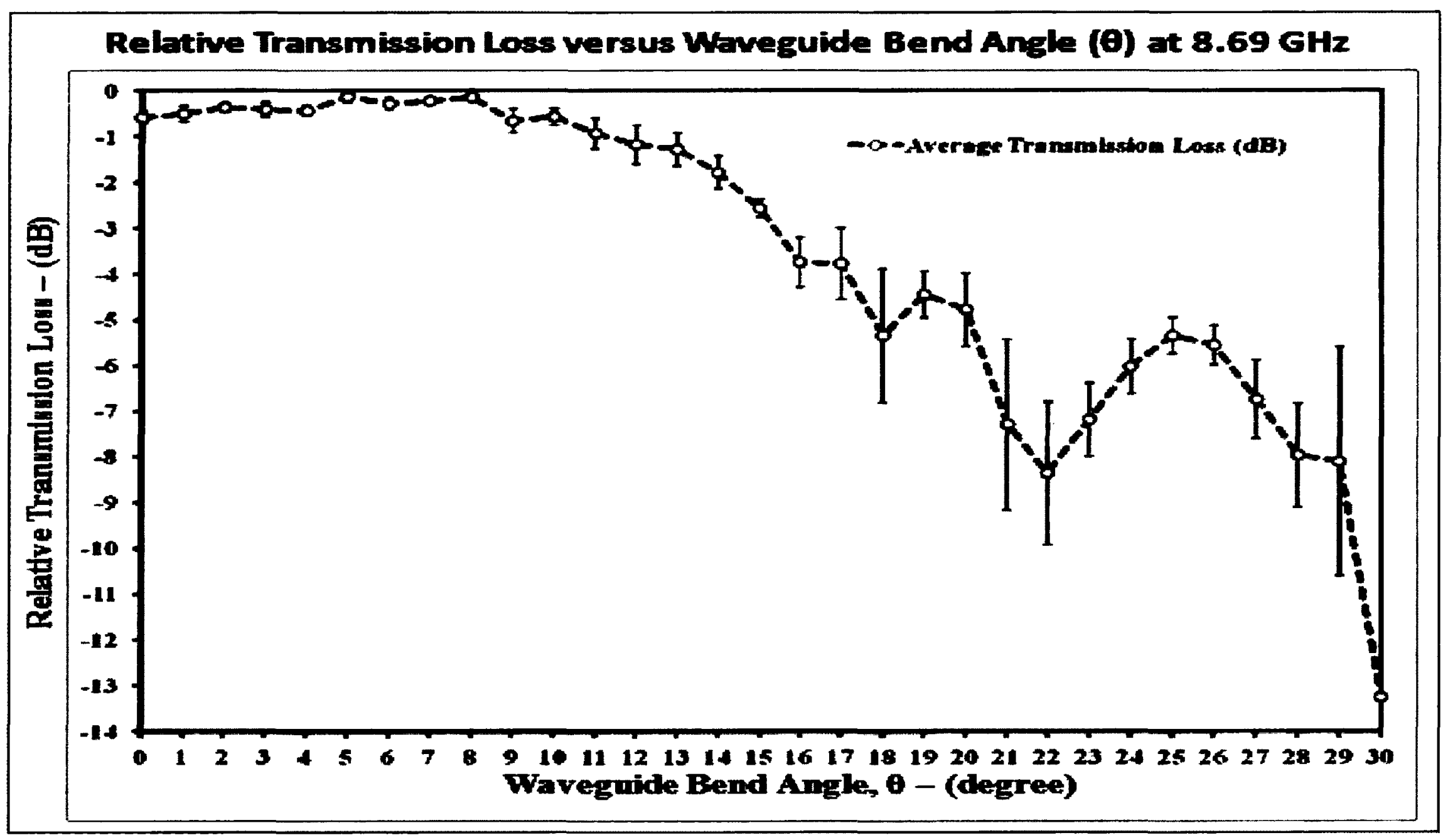

Figure D.5: Plot drawn at a particular frequency point, $\mathrm{f}=8.69 \mathrm{GHz}$ with different waveguide bend angle, $\theta$ versus relative transmission loss in microwave regime. 


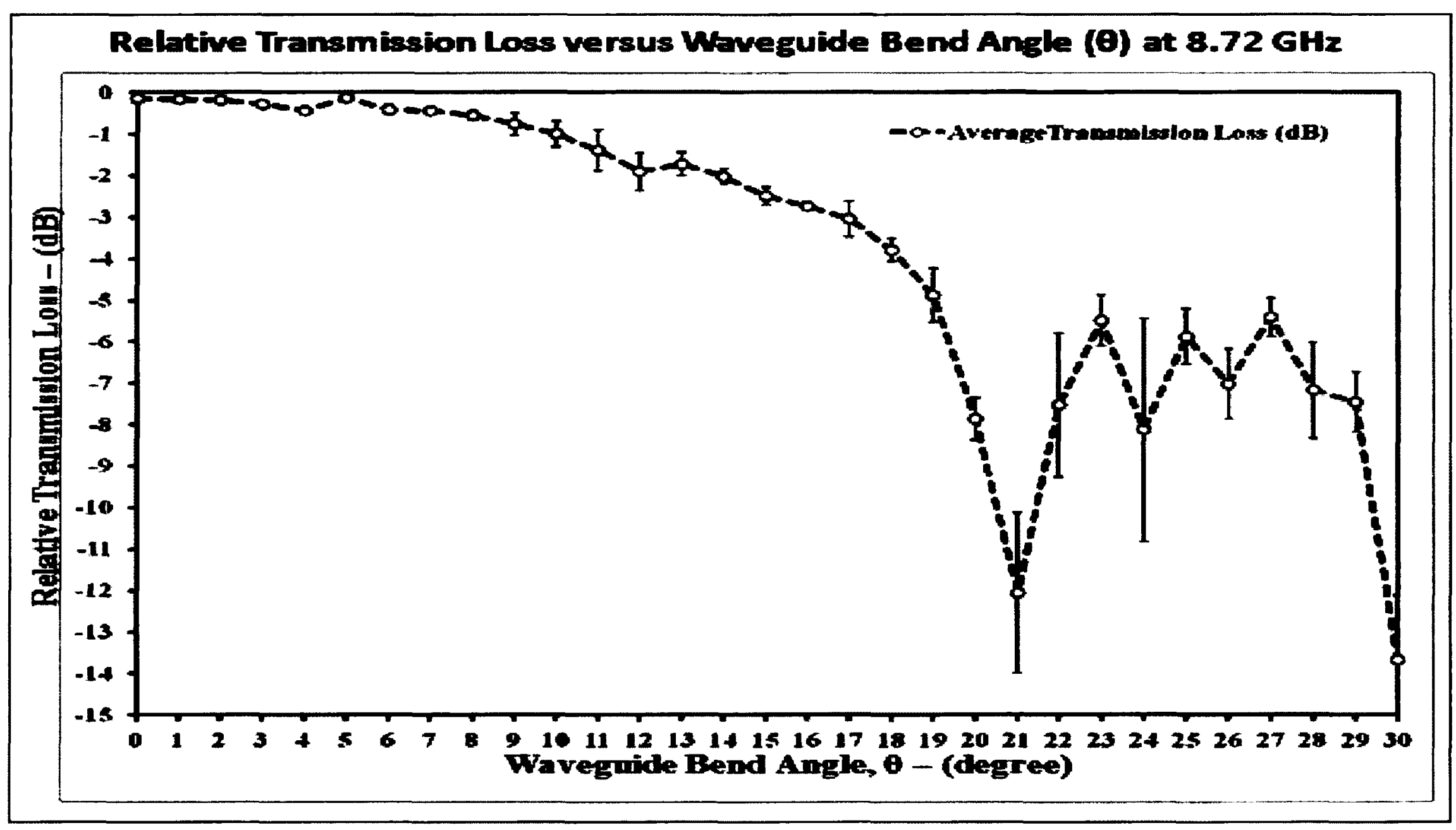

Figure D.6: Plot drawn at a particular frequency point, $\mathrm{f}=8.72 \mathrm{GHz}$ with different waveguide bend angle, $\theta$ versus relative transmission loss in microwave regime. 


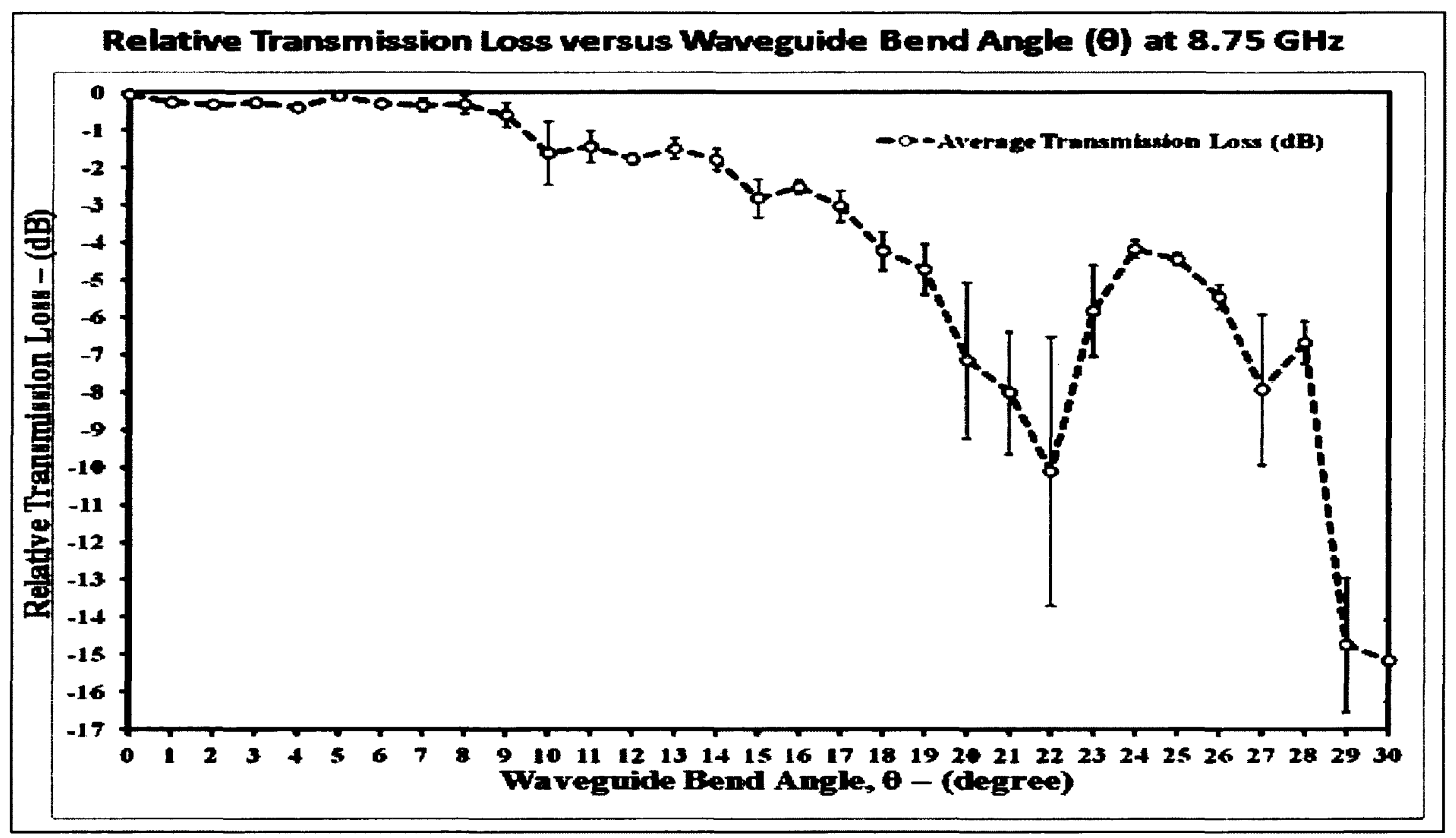

Figure D.7: Plot drawn at a particular frequency point, $f=8.75 \mathrm{GHz}$ with different waveguide bend angle, $\theta$ versus relative transmission loss in microwave regime. 


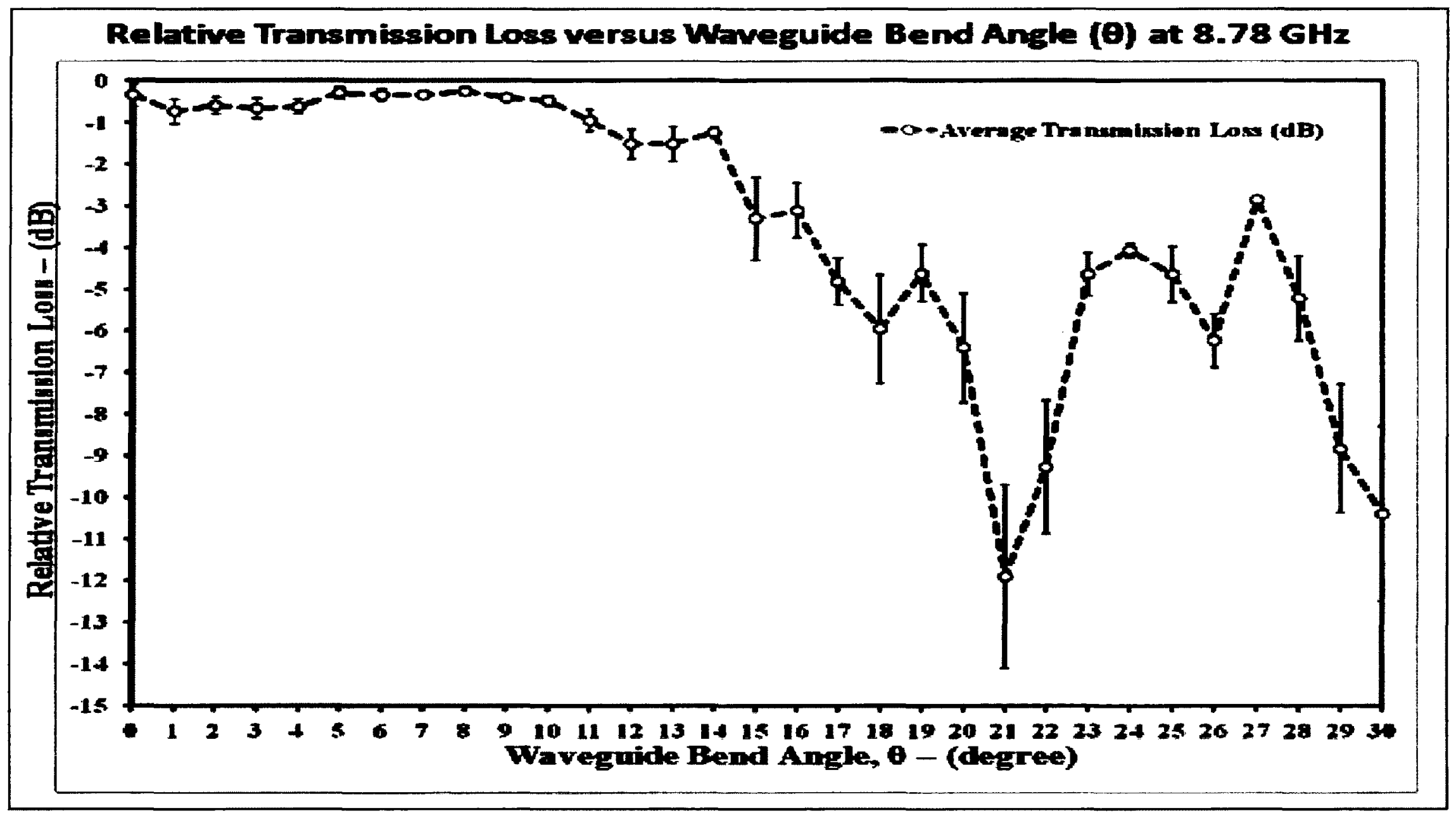

Figure D.8: Plot drawn at a particular frequency point, $\mathrm{f}=8.78 \mathrm{GHz}$ with different waveguide bend angle, $\theta$ versus relative transmission loss in microwave regime. 


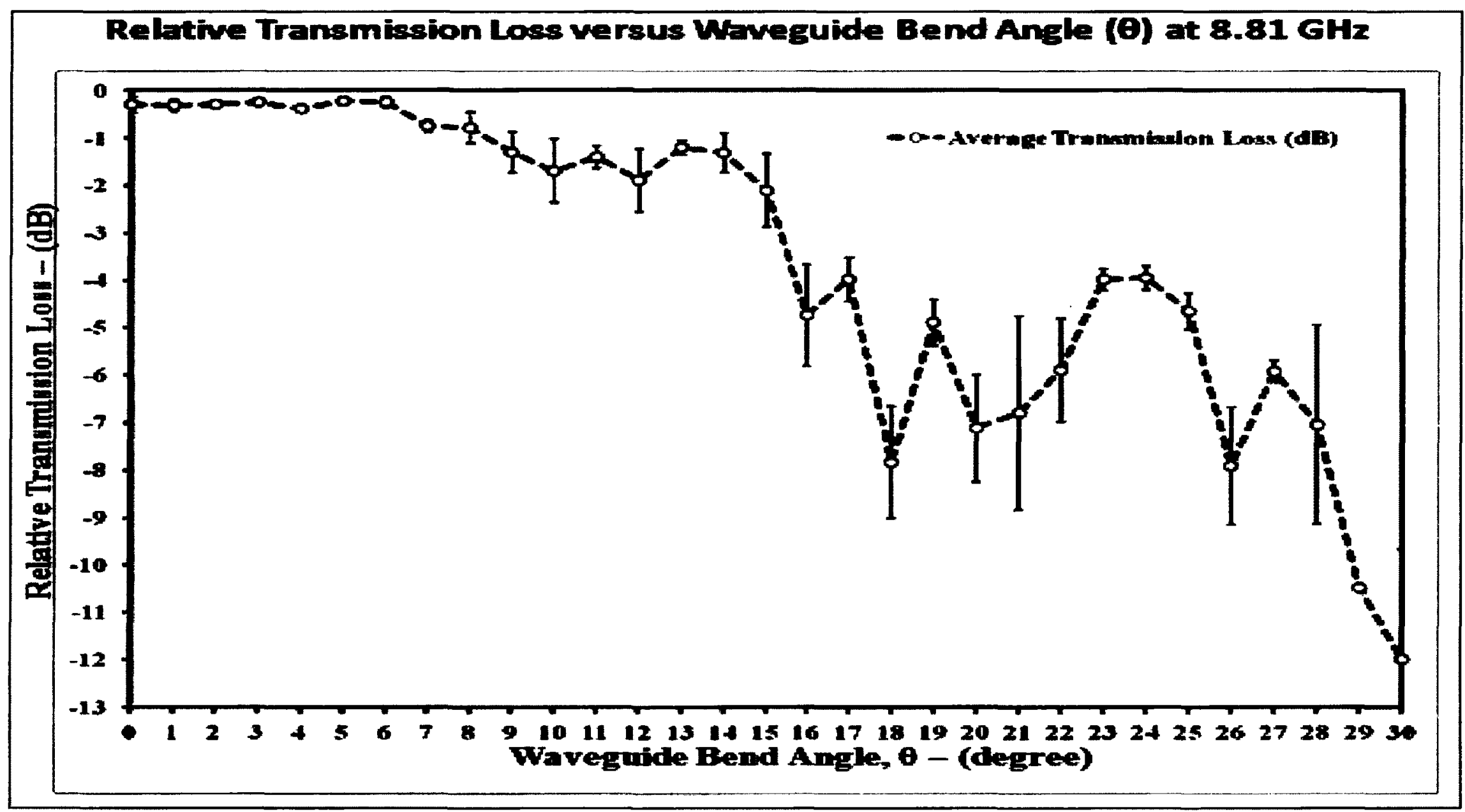

Figure D.9: Plot drawn at a particular frequency point, $\mathrm{f}=8.81 \mathrm{GHz}$ with different waveguide bend angle, $\theta$ versus relative transmission loss in microwave regime. 


\section{References}

[1] Joannopoulos D. J., Meade D. R., Winn N. J., Johnson G. S., "Photonic Crystals: Molding the Flow of Light", Princeton University Press (2008).

[2] Jean-Michel L., Henri B., Dominique P., Vincent B., Jean-Michel G.,and Daniel M., "Photonic Crystals: Towards Nanoscale Photonic Devices", Springer-Verlag Berlin Heidelberg (2005).

[3] Sullivan D M., "Electromagnetic Stimulation Using the FDTD Method", IEEE Press, New York (2000).

[4] Sakoda K, "Optical Properties of Photonic Crystals", Springer-Verlag New York Heidelberg (2001).

[5] Yablonovitch, E., "Inhibited Spontaneous Emission in Solid-State Physics and Electronics," Phys. Rev. Lett., Vol. 58, 2059-2062 (1987).

[6] S. John, "Strong Localization of Photons in Certain Disordered Dielectric Superlattices" Phys. Rev. Lett. 58, 2486 (1987).

[7] E. Ozbay, A. Abeyta, G. Tuttle, M. Tringides, R. Biswas, C. T. Chan, C. M. Soukoulis, and K. M. Ho, " Measurement of a three dimensional photonic band gap in a crystal structure made of dielectric rods", Phys. Rev. B, Vol. 50, pp. 1945-1948, (July 1994).

[8] E. Yablonovitch and T. J. Gmitter, "Photonic Band Structure: The Face-centeredcubic case", Phys. Rev. Lett., Vol. 63, pp. 1950-1953 (Oct. 1989). 
[9] E. Yablonovitch, T. J. Gmitter, R. D Meade, A. M. Rappe, K. D. Brommer and J. D. Joannopoulous, "Donor and Acceptor Modes in Photonic Band Structure", Phys. Rev. Lett. 67, 3380 (1991).

[10] K. Busch and S. John, "Photonic Band gap formation in certain self-organizing systems", Phys. Rev. E, Vol. 58, pp. 3896-3908 (Sept. 1998).

[11] S. Satpathy, Ze Zhang and M. R. Salehpour, "Theory of Photon Bands in three dimensional periodic dielectric structures", Phys. Rev. Lett., Vol. 64, pp. 1239-1242 (March 1990).

[12] J. Mizuguchi, Y. Tanaka, S. Tamura, and M. Notomi, " Focusing of light in a three dimensional cubic photonic crystal", Phys. Rev. B, Vol. 67, pp. 075109-1-7 (Feb. 2003). [13] K. M. Ho, C. T. Chan, and C. M. Soukoulis, "Existence of a photonic gap in periodic dielectric structures," Phys. Rev. Lett. 65, pp. 3152-3155 (Dec. 1990).

[14] M Schuster and N Klein, "Excitation of line and point defect modes in two dimensional electromagnetic bandgap structure for microwave frequencies", J. Phys. D: Appl. Phys. 37, pp. 374-378 (Jan. 2004).

[15] A. Matthews, X. H. Wang, Y. Kivshar, M. Gu, "Bandgap properties of two dimensional low index photonic crystal", Appl. Phys. Vol. 81, pp. 189-192 (Jan. 2005). [16] J. D Joannopoulos, Pierre R. Villeneuve and Shanhui Fan, "Photonic crystal:putting a new twist on light", Nature, Vol. 386, pp. 143-149 (March 1997).

[17] R. D. Meade, Karl D. Brommer, Andrew M. Rappe, and J.D Joannopoulos, "Existence of a photonic band gap in two dimensions", Appl. Phys. Lett. 61(4), pp. 495497 (July 1992). 
[18] K. M Leung and Y. F. Liu, "Full vector wave calculation of photonic band structures in Face-centered-cubic dielectric Media", Phys. Rev. Lett., Vol. 65 (21), pp. 2646-2649 (Nov. 1990).

[19] M. Plihal and A. A. Maradudin, "Photonic band structure of two dimensional systems: The triangular lattice", Phys. Rev. B, Vol. 44 (16), pp. 8565-8571 (Oct. 1991).

[20] D. R. Smith, R. Dalichaouch, N. Kroll and S. Schultz, "Photonic band structure and defects in one and two dimensions", J. Opt. Soc. Am. B, Vol. 10 (2), pp. 314-321 (Feb. 1993).

[21] M. Plihal, A. Shambrook, A. A. Maradudin, "Two Dimensional photonic band structures", Opt. Comm., Vol. 80 (3,4), pp. 199-204, (Jan. 1991).

[22] J. B. Pendry and A. MacKinnon, "Calculation of photon dispersion relations", Phys. Rev. Lett., Vol 69 (19), pp. $2772-2775$ (Nov. 1992).

[23] W. kohn, N. Rostoker, "Solution of the Schrodinger equation in periodic lattices with an application to metallic Lithium", Phys. Rev., Vol. 64 (5), pp. 1111-1120 (June 1954).

[24] R. D. Meade, K. D. Brommer, A. M. Rappe and J. D. Joannopoulus, "Electromagnetic Bloch waves at the surface of a Photonic crystal", Phys. Rev. B, Vol. 44 (19), pp. $10961-10964$ (Nov. 1991).

[25] W. M. Robertson, G. Arjavalingam, R. D. Meade, K. D. Brommer, A. M. Rappe, and J. D. Joannopoulos, "Measurement of photonic band structure in a two-dimensional periodic dielectric array "Phys. Rev. Lett. 68, 2023 (1992). 
[26] R. C. Gauthier, S. Newman and K. E Medri, "2D FDTD simulation of low loss small angle bend and $\mathrm{Y}$ branch configurations in a photonic crystal waveguide layout with a Mach-Zehnder device design configuration", Opt. Comm., Vol. 285, pp. 1976$1987(2011)$.

[27] Simon W. H. Tse, A. Karousos and Paul R. Young, "Broadband photonic bandgap waveguide", IEEE MTT-S International Microwave Symposium Digest, Vol. 3, pp. 2063-2066; (2004).

[28] M. Schuster and N. Klein, "Controlled excitation of electromagnetic bandgap line and point defect modes at microwave frequencies", J. Appl. Phys., Vol. 93(6), pp. 31823187 (March 2003).

[29] K. Sakoda, "Numerical study on localized defect modes in two dimensional triangular photonic crystals”, J. Appl. Phys., Vol. 84 (3), pp. 1210-1214 (August 1998).

[30] A. V. Dyogtyev, I. A. sukhoivanov and R. M. De La Rue, "Photonic band gap maps for different two dimensionally periodic photonic crystal structures", J. Appl. Phys., Vol. 107, pp. 013108-1-7, (Jan. 2010).

[31] S. R. Newman, R. C. Gauthier, "FDTD sources for localized state excitation in photonic crystals and photonic quasi-crystals", Proceedings of the SPIE, vol. 7223, pp. 72230R-72230R-10 (2009).

[32] J. Bai and G. Zhao, "Band gap of two dimensional photonic crystals of square lattices at Terahertz Frequencies", Proceedings of the SPIE, Vol. 7385, pp. 738517R738517R-10 (2009). 
[33] R. W. Ziolkowski and M. Tanaka, "FDTD analysis of PBG waveguides, power splitters and switches", Opt. and Quantum Elec., Vol. 31, pp. 843-855 (1999).

[34] D. W. Prather, S. Shi, J. Murakowski, G. J. Schneider, A. Sharkawy, C. Chen and B. Miao, "Photonic crystal structures and applications: Perspective, overview and deveoplment”, IEEE Quantum Elec., Vol. 12 (6), pp. 1416-1437, (Dec. 2006).

[35] A. Sharkawy, S. Shi and D. W. Prather, "Heterostructure photonic crystals: theory and applications", J. Opt. Soc. Am., Appl. Optics, Vol. 41 (34), pp. 7245-7253, (2002).

[36] H. Kurt and D. S. Citrin, "Photonic-Crystal heterostructure waveguides", IEEE Quantum Elec., Vol. 43 (1), pp. 78-84, (Jan. 2007).

[37] M. W. Kim, S. G. Lee, T. T. Kim, J. E. Kim, and H. Y. Park, "Experimental demonstration of bending and splitting of self-collimated beams in two dimensional photonic crystals", Appl. Phys. Lett., Vol. 90, pp. 113121R-113121R-3, (2007).

[38] N. Horiuchi, Y. Segawa, T. Nozokido, K. Mizuno, and H. Miyazaki, "High transmission waveguide with a small radius of curvature at a bend fabricated by use of a circular photonic crystal”, Opt. Soc. Am., Vol. 30 (9), pp. 973-975, (May, 2005).

[39] M. Tokushima, H. Kosaka, A. Tomita and H. Yamada, "Lightwave propogation through a $120^{\circ}$ sharply bent single-line-defect photonic crystal waveguide", Appl. Phys. Lett., Vol. 76 (8), pp. 952-954, (Dec. 1999).

[40] M. K. Moghaddam, M. M. Mirsalehi, A. R. Attari, "A 60" photonic crystal waveguide bend with improved transmission characteristics", Optica Applicata, Vol. XXXIX (2), pp. 307-317, (2009). 
[41] S. Y. Lin, E. Chow, V. Hietala, P. R. Villeneuve and J. D. Joannopoulos, "experimental demonstration of guiding and bending of electromagnetic waves in a photonic crystal", Science, Vol. 282 (5387), pp. 274-276, (Oct. 1998).

[42] M. Bayindir, E. Ozbay, M. M. Sigalas, C. M. Soukoulis, R. Biswas and K. M. Ho, "Guiding, bending and splitting of electromagnetic waves in highly confined photonic crystal waveguides", Phys. Rev. B, Vol. 63, pp. 081107(R)-081107(R)-4, (Feb. 2001).

[43] Z. huaiwu, "Photonic crystal waveguides in Terahertz Regime", $3^{\text {rd }}$ International Photonics and optoelectronics Meeting, J. of Physics, Series (276), pp. 1-13, (2010).

[44] S. Kim, I. Park, and H. Lim, "Design of Photonic crystal Splitters/Combiners", Proceedings of the SPIE, NanoPhotonic for Comm., Vol. 5597, pp. 129-135, (2004).

[45] S. Huang, J. Shi, D. Wang, W. Li, "Power splitters with different output power levels built with two dimensional photonic crystal", Proceedings of the SPIE, OE Lett.,Vol. 45 (2), pp.020503(R)-020503(R)-3, (Feb. 2006).

[46] M. Bayindir, B. Temelkuran, and E. Ozbay, "Photonic-crystal-based beam splitters", Appl. Phys. Lett., Vol. 77 (24), pp. 3902-3904, (Dec. 2000).

[47] B. Temelkuran, and E. Ozbay, "Experimental demonstration of photonic crystal based waveguides", Appl. Phys. Lett., vol. 74(4), 486-488, (Jan. 1999).

[48] A. Stiegelschmitt, A. Roosen, C. Ziegler, S. Martius, L. P. Schmidt, "Dielectric data of ceramic substrates at high frequencies", J. Eur. Ceramic Soc., Vol. 24, pp. 1463-1466, (2004).

[49] S. J. Penn, N. M. Alford, A. Templeton, X. Wang, M. Xu, M. Reece, K. Schrapel, "Effect of porosity and grain size on the microwave dielectric properties of Sintered Alumina", J. Am. Ceram. Soc., Vol. 80 (7), pp. 1885-1888, (1997). 
[50] M. Alzahrani, "Simultaneous Photonic and Phononic Band Gap, Defect States and Waveguides in Silicon-Polymer Composite", Master Thesis, Carleton university, Ottawa, (July 2012).

[51] Modinos, A., Stefanou, N., and Yannopapas, V., "Applications of the layer KKR method to photonic crystal”, Opt. Express 8, 197 (2001).

[52] Pendry, J. B., and Mackinnon, A. "Calculation of photon dispersion relations", Phys. Rev. Letter 69, 2772 (1992).

[53] http://www.doe.carleton.ca/ nagui/labequip/antenna/horn_antennas.pdf. (2013) [54]-http://www.electro-metrics.com/docs/datasheets/6/PDF-docs-100503/HTML-DataSheet-Quick-view-2/HORN/6961-New-Version.pdf (2013)

[55] Priya Rose T., E. Di Gennaro, G. Abbate and A. Andreone, "Isotropic properties of the photonic band gap in quasicrystal with low index contrast", Phys. Rev. B 84, 125111 (2011).

[56] Yee, K. S., "Numerical solution of initial boundary value problems involving Maxwell equations in isotropic media", IEEE, Vol. 14, No. 3, pp 302- 307 (1966).

[57] Berenger, P. J., "A perfectly matched layer for the absorption of electromagnetic waves", J. Computational Physic, 114, pp 185-200 (1994).

[58] User Guide Agilent Technologies;

'http://www.doe.carleton.ca/ nagui/labequip/netana/8720(22)ES_User's\%20Guide.pdf' (2013)

[59] Meade, D. R., Devenyi, A., Joannopoulos, J. D., Alerhand, O. L., and Smith, D. A., "Novel application of photonic band gap materials: lowloss bends and high Q cavities", J. App. Phys. 75, pp 4653-4755 (1994). 
[60] Mekis, A., Chen, J.C., Kurland, I., Fan, S., Villeneuve, P.R., and Joannopoulos, J. D., "High transmission through sharp bends in phtonic crystal waveguides", Phys. Rev. Lett. 77, pp. 3787-3790 (Oct. 1996).

[61] Johnson, S. G., Povinelli, Joannopoulos, J. D., "New photonic crystal system for integrated optics", Proceeding of SPIE, Vol. 4532, pp. 167- 179 (2001).

[62] Johnson, S. G., Chow, E. K. C., Lin, S. Y., Villeneuve, P.R., and Joannopoulos, J. D.,"Demostration of high waveguide bending efficiency $(>90 \%)$ in a photonic crystal slab at $1.5 \mu \mathrm{m}$ wavelengths", Proceeding of SPIE, Vol. 4283, pp. 453- 461 (2001).

[63] Chen, B., Tang, T., Chen, H., "Flexible photonic crystal waveguide branches with arbitrary branching angles", Optical Letter, Vol. 34, pp. 1952- 1954 (2009).

[64] Badaoui, H., Feham, M., Abri, M.,"Double bends and y shaped splitter design for integrated optics", Electromagnetic Research letter, Vol. 28, pp. 129- 138 (2012).

[65] Yonekura, J., Ikeda, M., Baba, T.,"Analysis of finite 2-D photonic crystal of columns and lightwave devices using scattering matric method", J. Lightwave Technology, Vol. 17, pp. 1500- 1508 (1999).

[66] Yang, W., Chen, X., Shi, X., Lu, W., "High transmission Y shaped waveguides in 2D photonic crystal with square lattice", PIERS Proceedings, pp. 631- 633 (2010).

[67] Sinha, R. K., Rawal, S., "Modeling and design of 2-D photonic crystal based Y type dual band wavelength multiplexer", Opt. Quant. Electron, Vol. 40, pp. 603-613 (2008).

[68] Chutinan A., Noda, S., "Waveguides and waveguide bends in two dimensional photonic crystal slabs", American Phys. Soc., Vol. B 62, pp. 4488- 4492 (2000). 
[69] Chutinan, A., Okana, M., and Noda, S., "Wider bandwidth with high transmission through waveguide bends in 2-D phtotonci crystal slabs", Applied Phys. Letter, Vol. 80, pp. 1698- 1700 (2002).

[70] Rauscher, K., Erni, D., Smajic, J., and Hafner, C., "Improved Transmission for $60^{\circ}$ photonic crystal waveguide bends", PIERS, pp. 25- 28 (2004).

[71] Zhang, Y., Li, B., "Ultracompact waveguide bends with simpke topology in 2-D photonic crystal slabs for optical communication wavelengths", Optical Letter, Vol. 32, pp. 787- 789 (2007).

[72] Miao, B., Chen, C., Shi, S., Murakowski, J., and Prather, D. W., "High efficiency broad band transmission through a double $60^{\circ}$ bend in a planar photonic crystal single line defect waveguide", IEEE Photonic Tech. Letter, Vol. 16, pp. 2469- 2471 (2004).

[73] Xiao, S., Qiu, M., "Study of transmission properties for waveguide bends by use of a circular photonic crystal", Phys. Letter, Vol. A 340, pp. 474- 479 (2005).

[74] Xing, P. F., Borel, P. I., Frandsen, L. H., "Optimization of bandwidth in $60^{\circ}$ photonic crystal waveguide bends", Optics Comm., Vol. 248, pp. 179- 184 (2005).

[75] Borel, P. I., Frandsen, L. H., Thorhauge, M., Cheng, J., Zhuang, Y., "Very low losses for TM polarized light in photonic crystal waveguides", Opt. Soc. of America, (2002).

[76] Borel, P. I., Frandsen, L. H., Thorhauge, M., Cheng, J., Zhuang, Y., "Propagation of TE and TM polarized light through smoothed sixty degree bends in planar photonic crystal waveguides", IEEE, pp. 677 (2003) 
[77] Olivier, S., Benisty, H., Smith, C. J. M., Rattier, M., Weisbuch, C.,"Transmission properties if 2-D photonic crystal channel waveguides", Opt. and Quant. Electron., Vol. 34, pp. 171-181 (2002).

[78] Lee, K. Y., Tsai, C. C., Weng, T. C., Kuo, Y. L., Kao, C. W., Chein, K. Y.,"Transmission characteristics of $90^{\circ}$ bent photonic crystal waveguide”, Fiber and Int. Optics, Vol. 25, pp. $29-40$ (2006). 